\title{
COMPARISON OF INHALABLE PARTICULATE AND TOTAL SUSPENDED PARTICULATE
}

\section{Document No. 83/12}

\author{
DEPOSITORY \\ JUL 61983 \\ UNIVERSITY OF ILLINOIS \\ AT URBANA.CHAMPAIGN
}

Energy and Natural Resources

James R. Thompson, Governor

Michael B. Witte, Director 
NOTICE: Return or renew all Library Materialst The Minimum Fee for each Lost Book is $\$ 50.00$.

The person charging this material is responsible for its return to the library from which it was withdrawn on or before the Latest Date stamped below.

Theft, mutilation, and underlining of books are reasons for disciplinary action and may result in dismissal from the University. To renew call Telephone Center, 333-8400

UNIVERSITY OF ILLINOIS LIBRARY AT URBANA-CHAMPAIGN

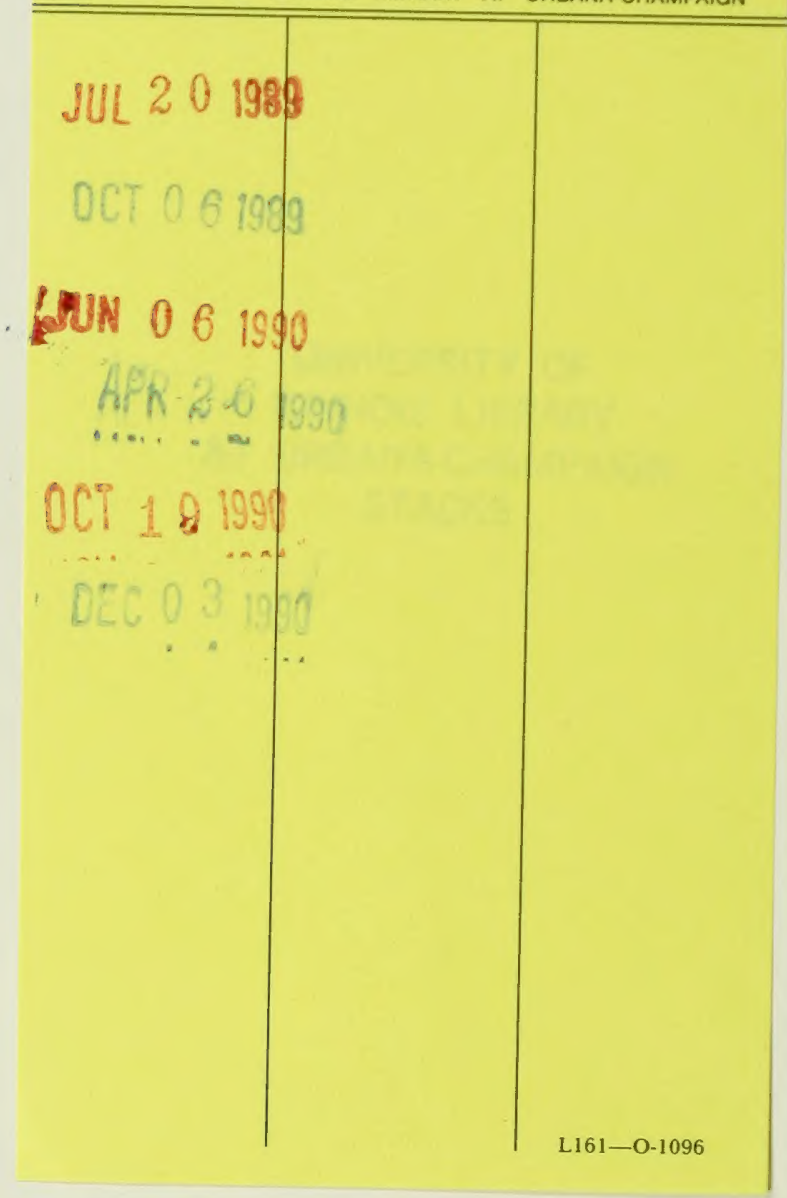




\section{COMPARISON OF INHALABLE PARTICULATE \\ AND TOTAL SUSPENDED PARTICULATE}

by

Robert Z. Muggli 1)

Principal Investigator

and

Mark E. Pulenik

John A. Brown

Ralph J. Hinch

Carol Rogers

Project No. 90.029

James R. Thompson, Governor

State of I11inois
Michael B. Witte, Director

Department of Energy and Natural Resources

1) Prepared under contract with the I11inois Department of Energy and Natural Resources as project number 90.029 ; to Walter C. McCrone Associates, Inc., Chicago, Illinois. 
3

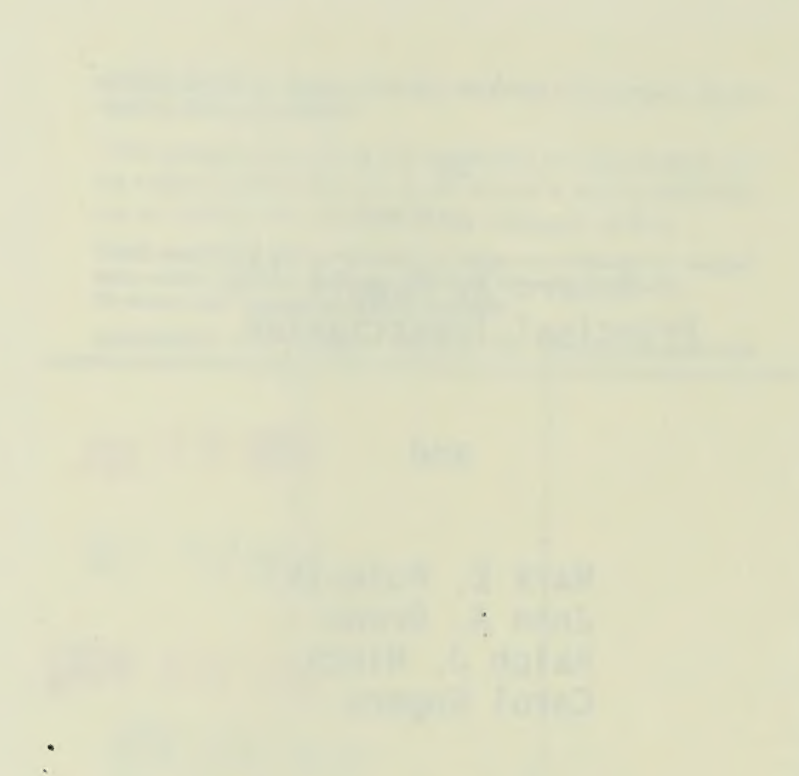

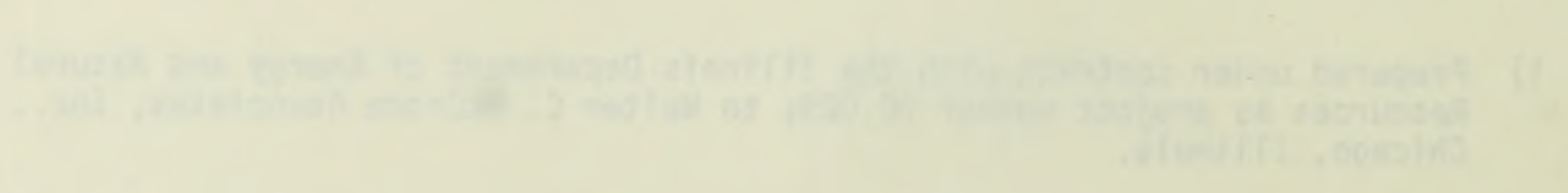


This report has been reviewed by the Department of Energy and Natural Resources and approved for publication. Views expressed are those of the contractor and do not necessarily reflect the position of DENR.

Printed by the Authority of the State of Illinois

$$
\begin{array}{ll}
\text { Date Printed: } & \text { March, } 1983 \\
\text { Quantity Printed: } & 150
\end{array}
$$

One of a series of research publications published since 1975. This

\begin{tabular}{|c|c|c|}
\hline & $\begin{array}{c}\text { Prior to } \\
\text { July, } 1982 \\
\end{array}$ & $\begin{array}{c}\text { After } \\
\text { July, } \quad 1982 \\
\end{array}$ \\
\hline Air Quality & - Green & Green \\
\hline Water & - Blue & Bive \\
\hline Environmental Health & - White & Grey \\
\hline Solid and Hazardous Waste & - White & 01 ive \\
\hline Economic Impact Study & - Buff & Brown \\
\hline Noise Management & - Buff & Orange \\
\hline Energy & - Cherry & Red \\
\hline Information Services & - Canary & Yellow \\
\hline
\end{tabular}
series includes the following categories and are color coded as follows:

I11inois Department of Energy and Natural Resources

Division of Policy and Planning

325 West Adams Street

Springfield, I17inois 62706

(217) $785-2800$ 


\section{ACKNOWLEDGEMENTS}

We would like to acknowledge the assistance of the staff of the Illinois Environmental Protection Agency and especially Dave Kolaz. They provided the high-volume filters for analysis, the information on the site locale and exposure information. We would also like to thank William Murphy of ENR for his assistance by discussion and his efforts as Project Director. 
Digitized by the Internet Archive in 2013 


\section{ABSTRACT}

An optical and electron optical examination of filters from seven sources showed that the major difference between inhalable and total suspended particulate is the loss of the large mineral, metal and metal oxide particles. As particulate loading of the atmosphere increases, the ratio of fines (<about $2 \mu \mathrm{m}$ ) in the sample also generaliy increases. 

Acknowledgment

Abstract

List of Figures

Summary

Introduction

Background

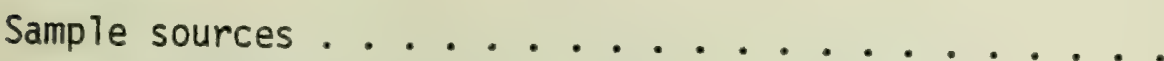

Analytical Procedures

Polarized Light Microscopy . . . . . . . . . . . . . 5

Low Temperature Ashing . . . . . . . . . . . . . . 6

Scanning Electron Micrographs............. 7

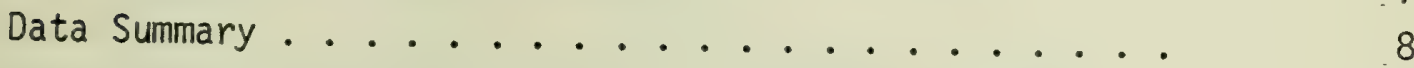

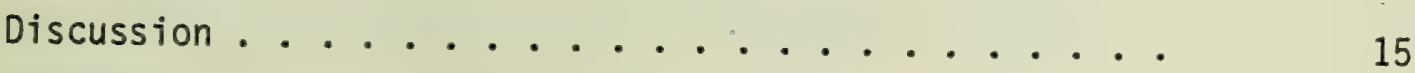

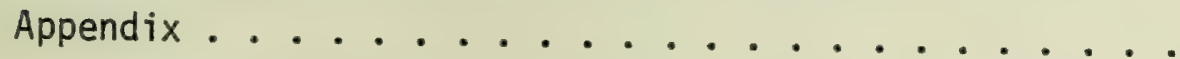
7

Site, Description, Diagrams and Data Sheets from Filter Analysis

A. Springfield..............

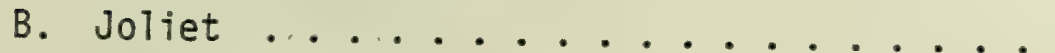

C. Rockford

D. East Moline............ D D

E. Peoria .............. . . . E EI

F. Granite City (Fire Station AI) ....... F1

G. Granite City (Dallas residence) ....... . G1

H. Scanning electron micrographs from ultrasonification study ......... 

Number

1 Overview of the Inhalable Particulate Network

2 Springfield Filter Samples and Their Particle Loadings

3 Joliet Filter Samples and Their Particle Loadings

4 Rockford Filter Samples and Their Particle Loadings

5 East Moline Filter Samples and Their Particle Loadings

6 Peoria Filter Samples and Their Particle Loadings

7 Granite City (FSI) Filter Samples and Their Particle Loadings

8 Granite City (Dallas Residence) Filter Samples and Their Particle Loadings 

A short study by scanning electron microscopy showed that the small particles on the glass fibers of a $\mathrm{Hi}-\mathrm{vol}$ filter are strongly attracted to the fibers. The examination also indicated that the ratio of small particles in the atmosphere generally increases as the dust loading of the atmosphere increases. As has been shown previously, this total loading typically increases as the sites become more industrial. The makekup of inhalable and total suspended particulate samples are generally quite similar. The major differences are those which result from the loss of the larger mineral, metal and metal oxide particles. Thus total suspended particulate data are still useful and will, in a general way, correlate with data collected on a sampler with a size selective inlet. 

COMPARISON OF INHALABLE PARTICULATE

AND TOTAL SUSPENDED PARTICULATE

\section{INTRODUCTION}

In accord with the $1977 \mathrm{Clean}$ Air Act Amendments, the USEPA is establishing a nation-wide inhalable particulate standard to assess the air quality levels of particles less than 15 micrometers in size. As it appeared that an inhalable standard would be proposed and promulgated within the next two years, the Illinois EPA desired to assess the effect that such a standard would have on Illinois. To support this evaluation the IIlinois EPA requested an assessment of the composition, concentration and size range of both total suspended particulate and inhalable particulate. By assessing these particulates the 111 inois EPA will be better prepared to intelligently comment on any proposed USEPA inhalable particulate standard and also to be better prepared to assess its impact on Illinois.

- Inhalable particulate (IP) monitors were located near total suspended particulate (TSP) monitors at seven sites in Illinois. (One each in East Moline, Peoria, Joliet, Springfield, Rockford; two in Granite City). Some of the filter pairs from these monitors were to be examined with optical and electron microscopy and low temperature ashing to produce data for comparison of the relationship of IP to TSP. 

The 1977 Clean Air Act Amendments required that ambient pollutant standards be revised in 1980 . It was determined that TSP measurements included particles that are inhalable (less than 15 micrometers) and not inhalable (greater than 15 micrometers). To assess those particulates that are a possible health hazard a network of Inhalable Particulate monitoring stations were established. An overview of operations of the Inhalable Particulate network prepared on June 10th 1979 by Charles Rodes of the Environmental Monitoring and Support Laboratory, Research Triangle Park, NC is shown in Figure 1.

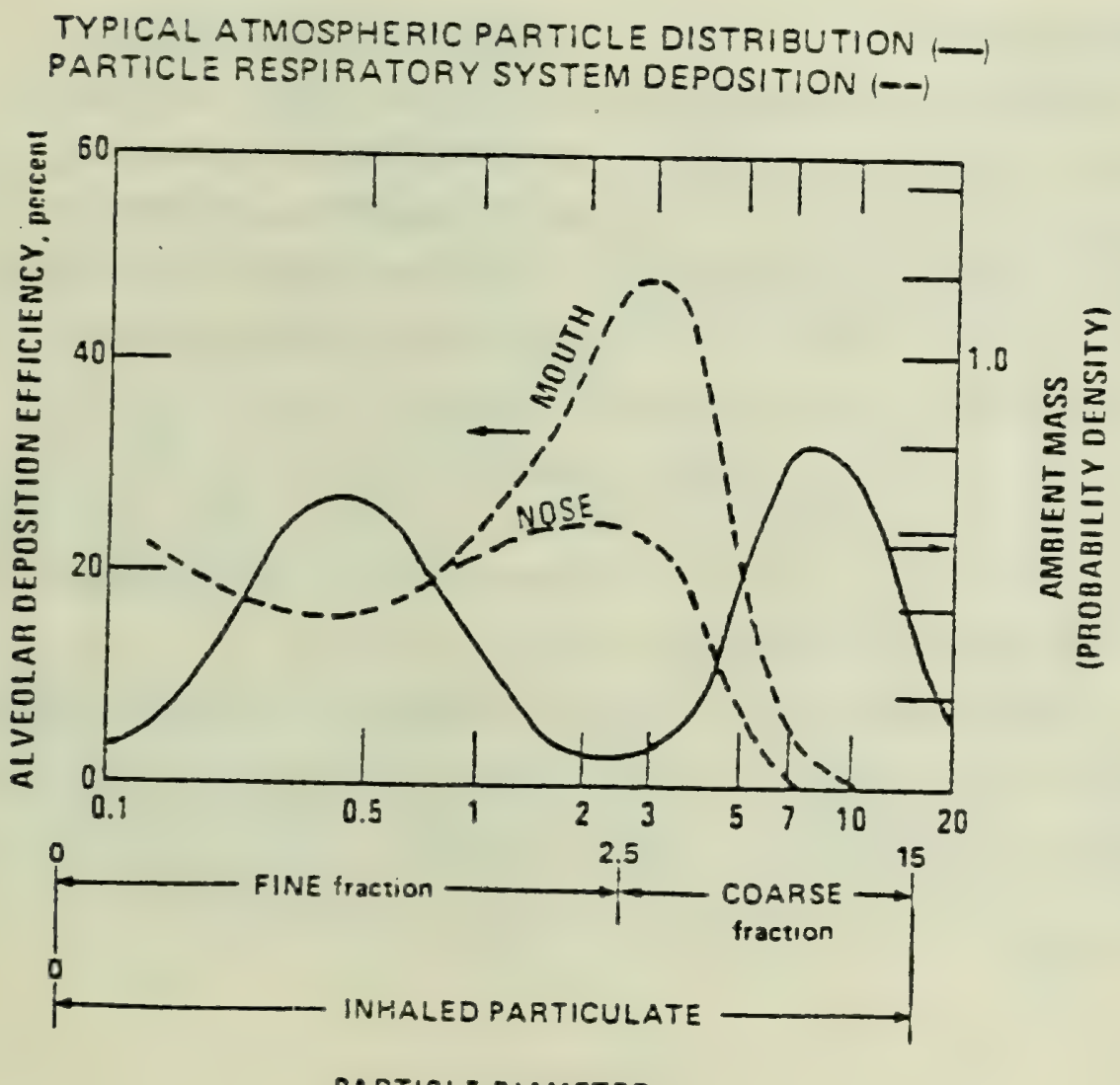

Figure 1 
This figure shows that two size fractions are typically present in TSP, a course fraction of about 30 to $2.5 \mu \mathrm{m}$ with a maxima at about 8 to 10 um and a fine fraction from 2.5 to 0.1 micrometers with a maxima of about $0.5 \mu \mathrm{m}$. The figure also notes that the typical alveolar depositable fraction is generally that portion with particle diameters of less than about 5 to 10 micrometers.

A figure on page A66 of a report of the 146 Air Quality Technical Committee in the American Industrial Hygiene Journal (Vol. 42, May 1981) shows approximately the same data for the alveolar fraction and in addition notes that a tracheobronchial fraction of about 15 or 20 to 2 micrometers also exists.

It was with this data in mind that the $15 \mu \mathrm{m}$ limit for inhalable particulate was estabiished.

\section{Sample Sources}

Samples from seven sources were analyzed. These represented industrial, commercial and residential areas.

Two sites were described as being mainly residential. Both were near some commercial as well as agricultural areas. These were the Springfield and Joliet collection sites.

The other sites are more industrially oriented. The Rockford site is surrounded by a residential area and has many industrial sites nearby (less than one mile). The East Moline site is somewhat simiar, but appears to have more commercial and less industrial sources nearby.

The Peoria site is listed as commercial and residential, but it is near (a few miles) to many rather large industrial sources. The two Granite city sites are mainly industrial with the Fire Station site being under some commercial influence. 
Both of the Granite City sites are listed as industrial with the fire station being more influenced by the commercial and residential areas near the site. The Dallas residence in Granite City is noted as industrial although it too does have some element of residential influence. All sites are near moderately traveled roads except for the Peoria site which is near two heavily traveled streets.

All collectors were placed on low buildings with typically minor or no wind obstructions near the collection sites. The filter samples were collected in 1981.

\section{ANALYTICAL PROCEDURES}

\section{Polarized Light Microscopy}

Polarized light microscopy was employed to identify the types of particles on the filters received for this study. Only a brief description will be presented here as the techniques have been fully explained in The Particle Atlas published by Ann Arbor Science Publishers.

The optical microscope is unique in its ability to allow an individual to identify specific types of chemical entities as to their particular sources for example; diatoms, beach sand, fiberglass, broken glass and crushed quartz. All are silica yet their different sources are apparent. Another example is that of combustion sources. Particles from $0 i 1$ fired boilers, high efficiency coal fired boilers, incinerators, and coal fired boilers 911 have different properties and thus can be distinguished from one another. 
The filters were examined at low power with a stereomicroscope to ascertain that the distribution of the collected sample was uniform. Several samples were then removed from the filter at random, composited and examined with the polarized light microscope. The optical properties such as color, texture, shape, refractive index, birefringence, transparency, crystal class, habit, pleochroism, cleavage and extinction typically yield a unique set of properties for each specific type of particle. Each particle was examined for type and its size was noted.

The size determinations were made by comparing the Martin's diameter of each particle to the circles or rectangles on a Porton graticule. Martin's diameter is the longest horizontal cord through a particle as it randomly lies in the field of view. A porton graticule is an eyepiece scale containing a series of circles and rectangles increasing in size by the $\sqrt{2}$. This scale was calibrated with a stage micrometer.

When all of the particles in a specific area of the microscopical preparation were identified, another area was chosen and examined in a similar fashion. This continued until 500 to 1000 particles had been identified and sized. The data were then mathematically computed to yield the data sheets attached in the Appendix.

Low Temperature Ashing

Sections of each filter were removed for analysis. A $5.1 \times 3.7 \mathrm{~cm}$ section was cut for analysis, dessicated for 12 hours and then weighed. They were ashed for at least three hours in Plasmod LTA and then re-weighed. Each sample represented $1 / 22$ of the entire filter area. Weight losses were reproducable to $\pm 0.1 \mathrm{mg}$ on three blanks cut from three different unused filters. 


\section{Scanning Electron Micrographs}

Small samples were cut from each filter and mounted on aluminum stubs with double-sided tape. The edges of the samples were painted with conductive paint and the samples were coated with gold. The coated specimens were examined with a scanning electron microscope at a variety of magnifications and representative portions were photographed at 1200 and $60000 x$ magnifications. The prints are presented in the Appendix.

A small study of the effectiveness of removal of particulate from $\mathrm{Hi}-\mathrm{vol}$ filters by solvents and ultrasonification was carried out. Small pieces of Hi-vol filter samples were cut and placed in a test tube with several milliliters of solvent. The tube was placed in an ultrasonic bath for twenty minutes. The piece of filter was retrieved and examined, as above, with a scanning electron microscope. The water layer from the sample extracted with water was filtered through a Nuclepore filter and that was also examined with a scanning electron microscope.

\section{Sample Description}

Figures 2-8 show each of the samples and their particle loadings. A graphical presentation which depicts the loading for each sample and the respective loadings per category ( $M=$ minerals, $\mathrm{C}=$ combustion products, $\mathrm{B}=$ biological and $0=$ other, miscellaneous) is shown on the optical microscopy sheets within each Appendix. 


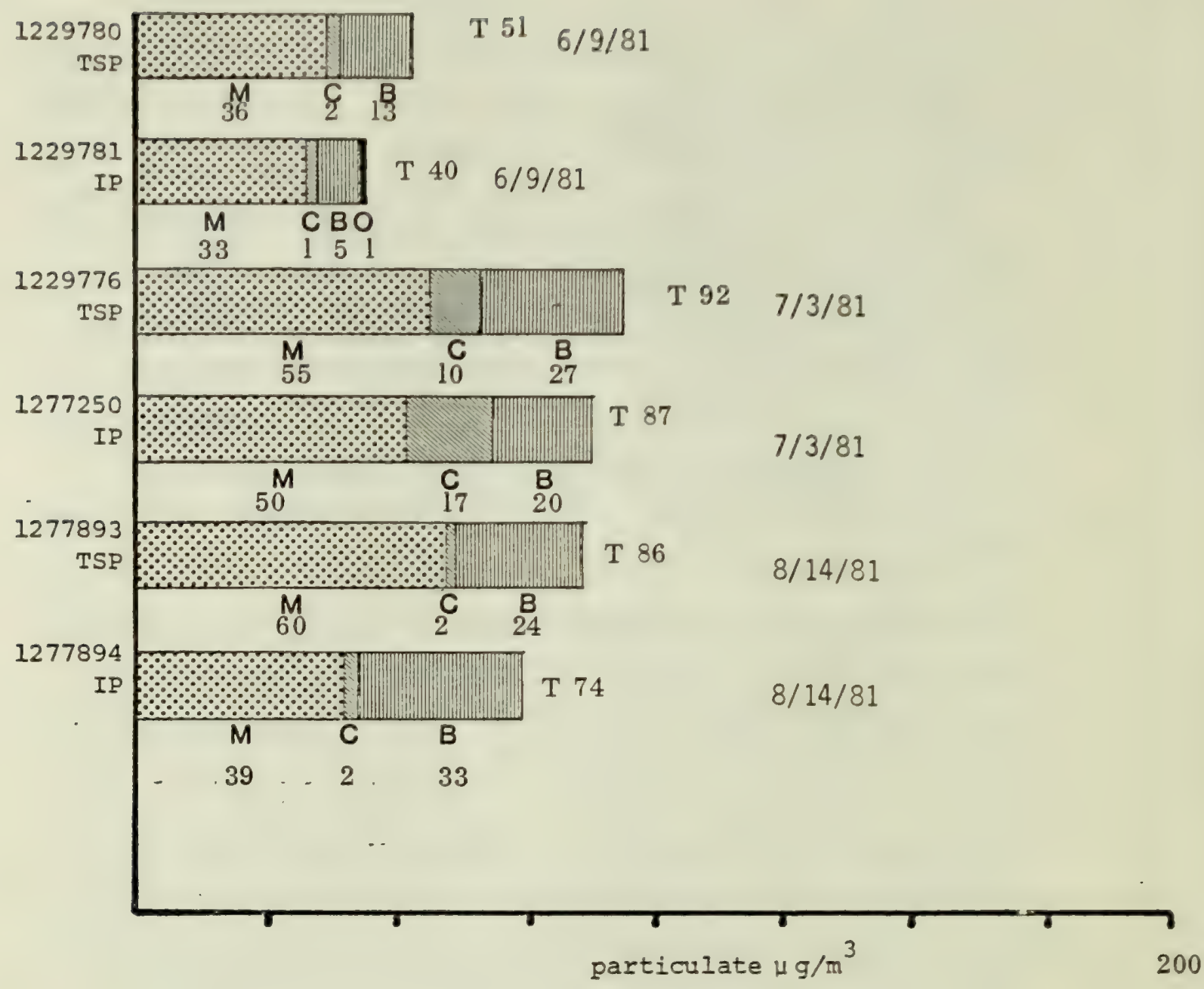

These samples were collected during June, July and August. The collection site was rural residential. The loading of the filters is quite low and the major components of the samples were minerals and moderate amounts of biologicals. Compared to the other sites the biological contents were generally higher. There is relatively little difference in the total loading and ratio of particulate types between the total suspended particulate and inhalable particulate. The SEM analyses show that only a few particulates are present in the sub-optical range $(<1$ to $2 \mu \mathrm{m})$.

Figure 2 


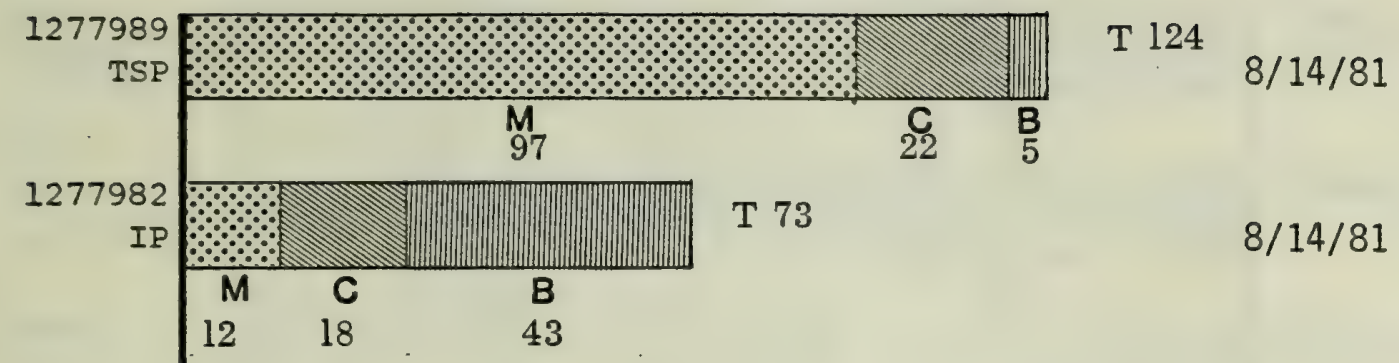

The samples were collected during August. Moderate loading of the filters was experienced, consisting mainly of minerais with moderate combustion and biological components. These were typical for the semi-rural residential character of the site. The inhalable particulate was quite a bit lower than the total suspended particulate because of the large size of the mineral particles. The SEM analyses showed that moderate amounts of the particulate was sub-optical.

Figure 3 


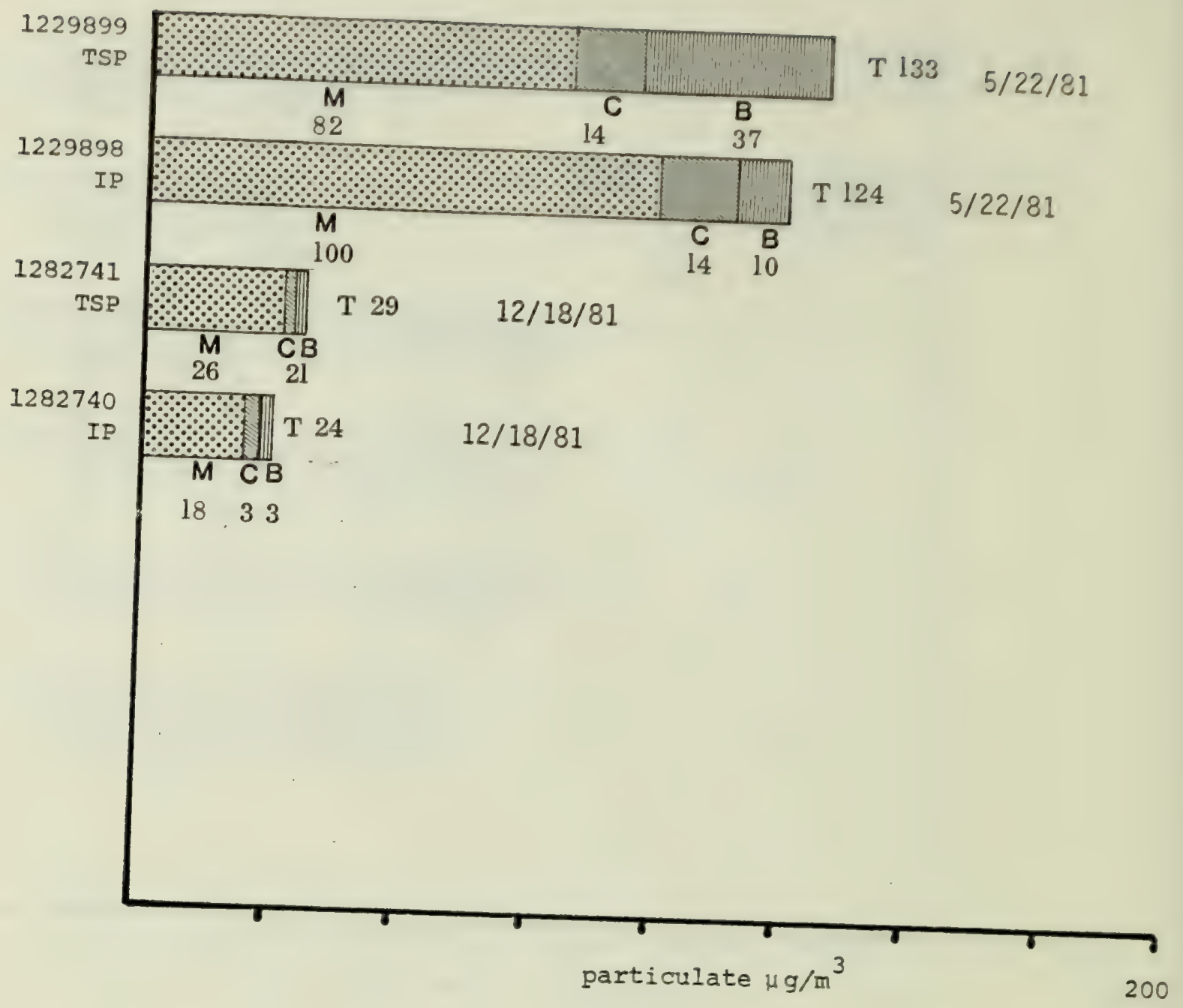

This site was reported as being residential with industry nearby. was very low. Both collections showed ring and that from December difference in loading and ratio showed relatively littie inhalable and total suspended particulatelate types between mostly mineral with moderate amounts of combustion collection was particulates. In the winter the major particulat and biological There was a fairly large ratio of sub-optical particles mineral. from both the summer and winter collections.

Figure 4 


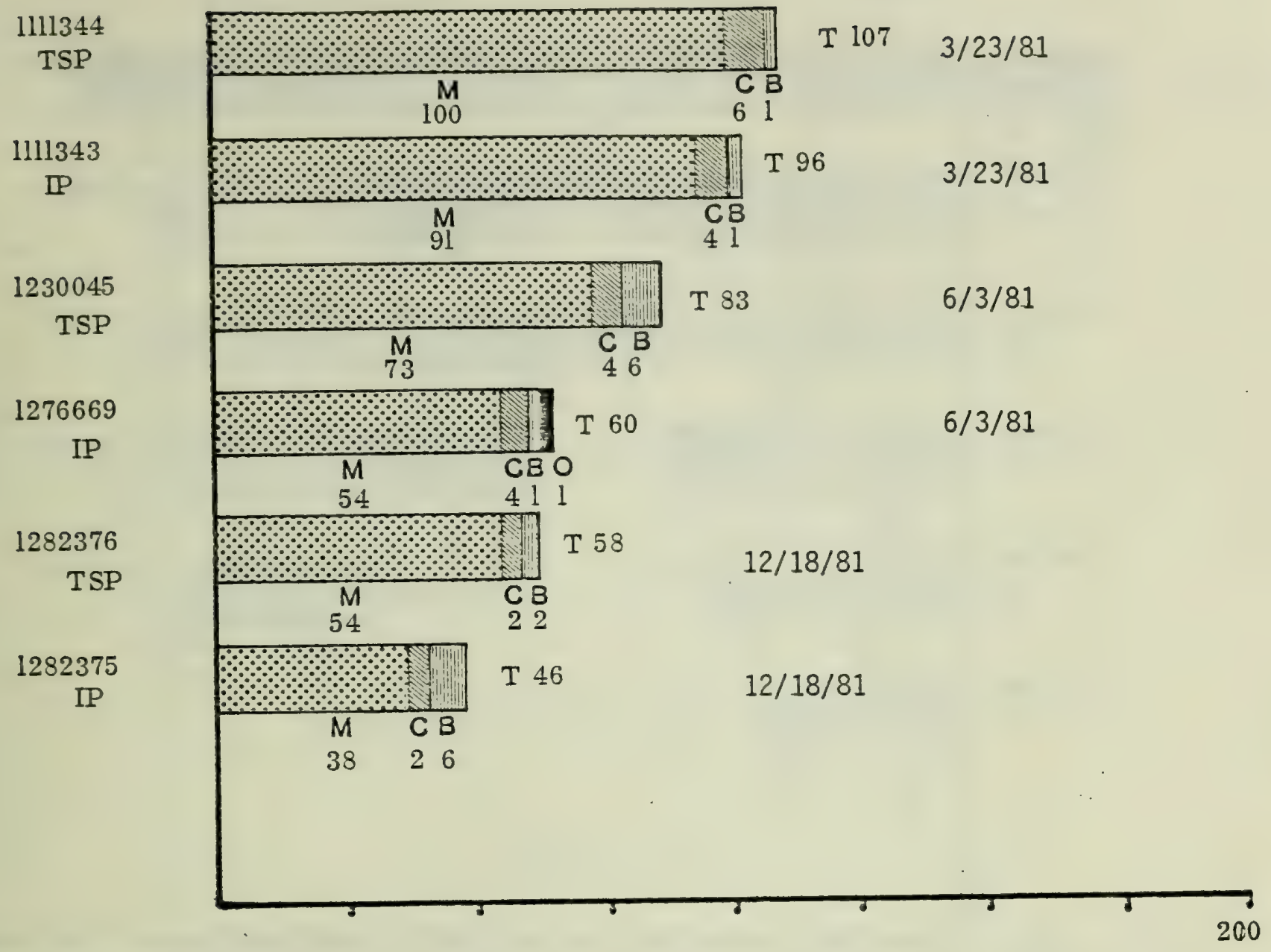

This collection site was at a combination residential and commercial location. The analyzed samples were taken during March, June and December. Moderate loading was observed during March sampling, lower loading for the June sampling and low loading for the December sampling period. In all cases most of the particulate was mineral. Only moderately lower levels of particulate were collected on the filters in the inhalable particulate samplers. Many fines and aggregates of fines were noted during the SEM examination, especialiy on the spring and summer sample filters.

Figure 5 


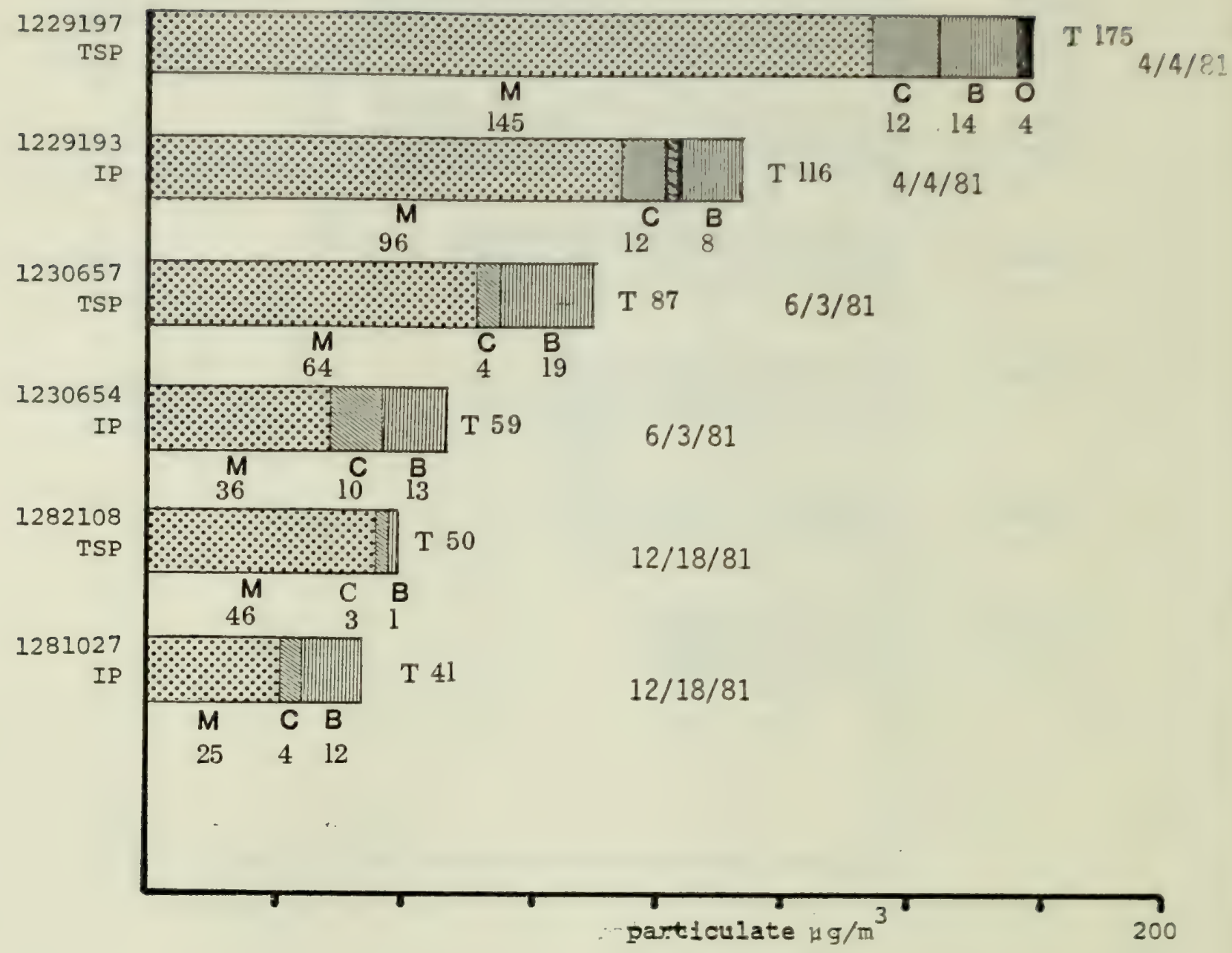

The Peoria site was noted as a residential site, but it is near commercial and industrial installations as well as heavily travelled streets. Samples were analyzed from April, June and December with moderately high, moderate and low dust loadings respectively. Again minerals were the major component. The large size of the minerals in the April and June collection created a large difference between the inhalable and total suspended particulates for those collections. Higher metal and iron oxide levels were noted than in the more residential samples. Combustion product levels are also somewhat more elevated. Moderate to moderately high levels of agglomerates of fines and fines on the sample filters were seen during the SEM examinations.

Figure 6 


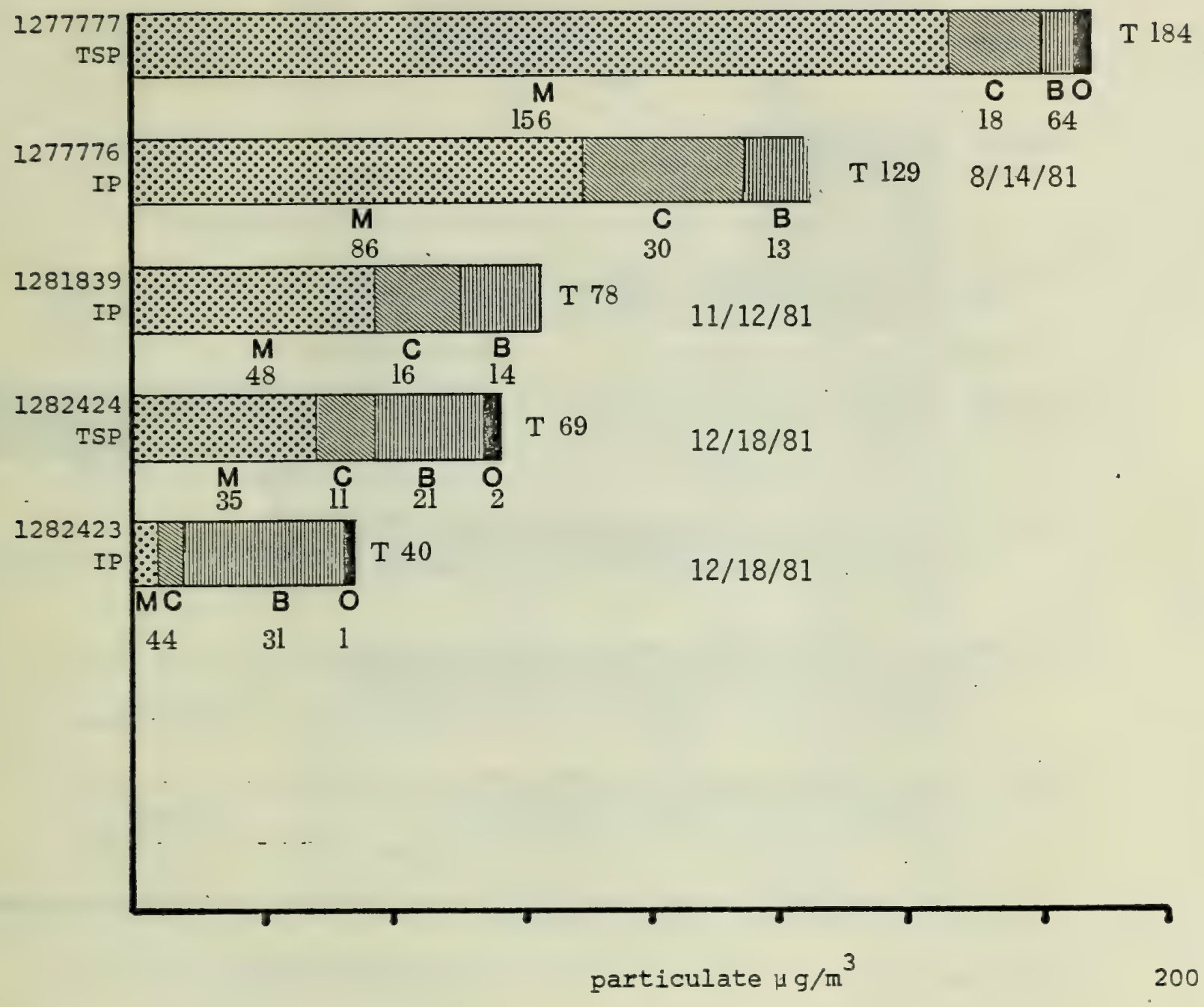

This site was recorded as industrial, but it is also near some comnercial and industrial facilities. The studied samples were collected during August, November and December. The data are quite similar to those from Peoria, as one might expect from the similarity of the locations. That is, the August loadings are moderately high and the winter samples show moderate levels of particulate. These samples contain generally higher levels of combustion, metal and iron oxide particulates than most of the other samples. The December samples contain soft coal. The large size of the minerals makes the August inhalable particulate sample smaller than the companion total suspended particulate sample. All samples show a high proportion of fines under SEM examination. 


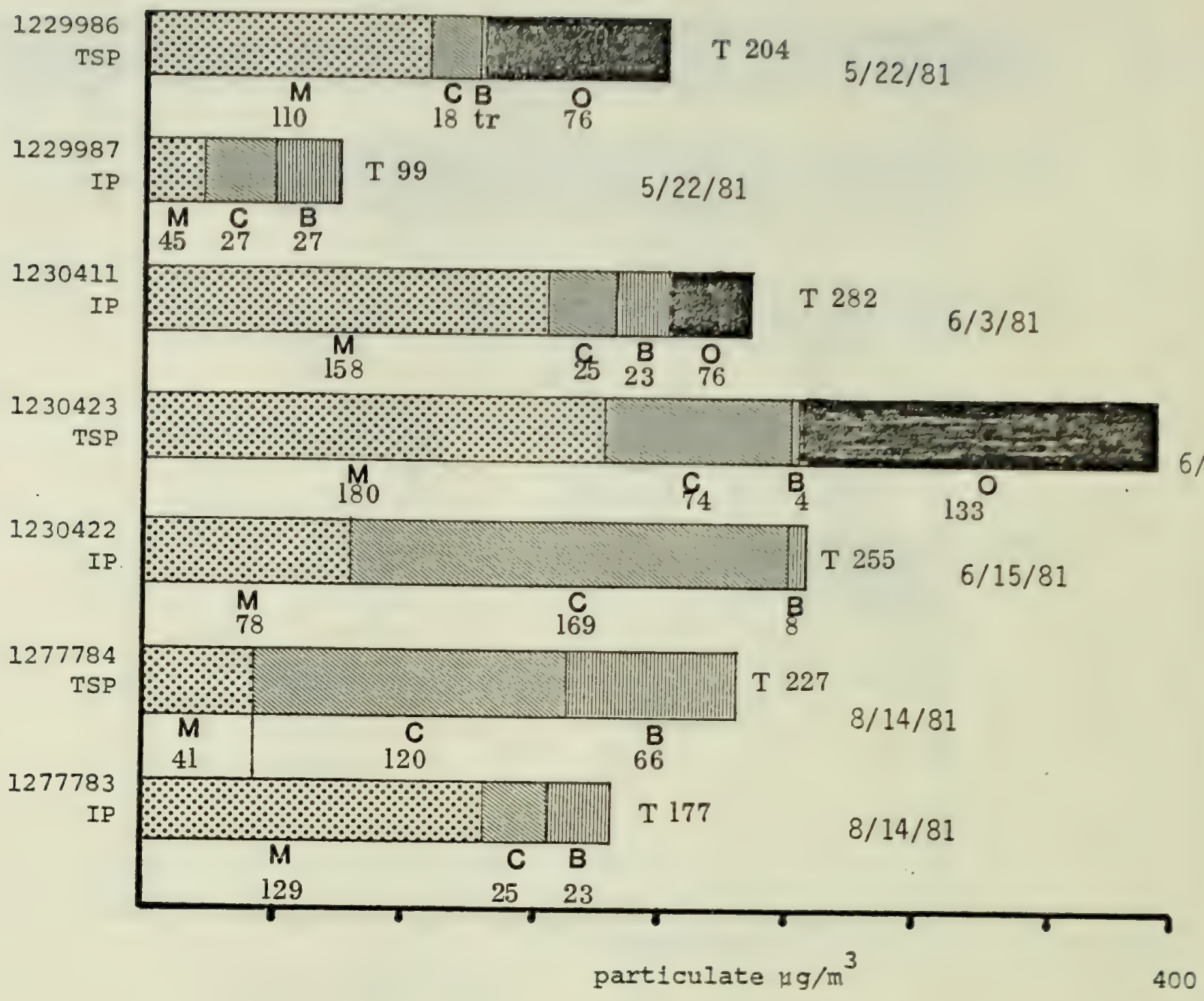

This site is a residential location surrounded by industrial facilities. The samples examined were collected during May, June and August. The samples are moderately to heavily loaded. Little biological particulate is present. Minerals, especially iron oxide, combustion products and metals are the major particulate types. The major differences between the inhalable and total suspended particulate samples are the greatly reduced levels of iron oxides and metals in the inhalable fraction for the May and June samples and the greatly reduced soot levels in the August inhalable particulate sample. All samples show moderately high to high levels of sub-optical particles in the SEM examinations.

Figure 8 


\section{DISCUSSION}

The samples for polarized light microscopical (PLM) analysis were removed from the filters with a small droplet of Aroclor - on a tungsten needle. This has been previously shown to be a reproducible technique. The sample thus obtained has also been found to be representative of the deposit on the filter (see the Particle Atlas, op. cit).

For scanning electron microscopical analysis (SEM) a small portion of the filter is typically removed and after metallization, the particles are examined in situ. It was felt that it might be advantageous to examine the particles on a Nuclepore: filter surface so that the glass fibers of the Hi-Vol filter would not be present in the field of view. Such a procedure would be valid only if reproducible and representative samples could be produced.

The SEM micrographs in Appendix $H$, pages $\mathrm{H} 1$ through $\mathrm{H} 6$, demonstrate the difficulty of such a procedure. Few particles less than about $10 \mathrm{pm}$ were removed from the filters in any of the solvents tested, even after twenty minutes of ultrasonification. This can be seen by an examination of the micrographs of the various Hi-Vol filter samples and also the deposit on the Nuclepore filter. Additionally, so many small fragments of glass fibers are present on the Nuclepore filter surface, even if the small particles were removed and deposited, the fiber fragments would be even more distracting than fibers of the original filters. 
The tenacity of the particles to the fibers in the presence of the solvent and ultrasonification is quite strong. If the "chemical glue" were either organic liquids or inorganic salts such as sulfates, the ultrasonification procedure would be expected to remove the particles. The forces that bind the particles must therefore be more fundamental, e.g., electrostatic attraction.

The examination of the filters by PLM and SEM showed that minerals and biological particulates are most common at the rural and residential sites. As the sites become more commercial and industrial the level of combustion products, iron oxides and metal particulates increase. In general, when large particles of minerals and metals are present, the inhalable particulate levels are lower than the total suspended particulate levels. These two types of particulates most often represent the differences between the two types of fractions.

The biological particulate fraction not only increased in the inhalable particulate because of the loss of minerals and metals, but because the low density of the biologicals allows oversize particles of this type to be collected rather than rejected by the size selective inlet to the sample collector.

The correlation of the low temperature ashing data with the mass of microscopically determined biological fraction is poor. There is only a general correspondence between the two values and at times the percent loss by low temperature ashing is considerably lower or higher than the weight percent of that particular fraction. This may partially be caused by the presence of non-particulate deposits.

Particulate levels increase as the collection sites become more industrial. As the suspended solids increase the ratio of sub-optical particulate also increases in both the inhalable and total suspended fractions. 
Solids levels are lowest in the winter and highest in the summer. Often the inhalable and total suspended fractions do not differ greatly either in quantity or ratio of particulate types.

Thus, although it is better to record data that relates more directly to the fraction that is physiologically trapped, the data derived by the previous sampling of total suspended solids are still of value because of the general similarities of the two types of fractions. 
A. Site Identification

1. City: Springfield

2. Site Name and Address: IEPA Building, 2200 Churchill Road

3. County: Sangamon Township: Springfield

4. USGS Topographical Map Name and Scale: Springfield West, IL, 1:24,000

5. Site Elevation (Feet): 580

B. Site $\mathrm{Cl}$ assification/Representativeness

1. Dominating Influence on Site: Residential, Undeveloped

C. Source Impact

1. Stationary Sources that may Influence Site:

Name of

Source

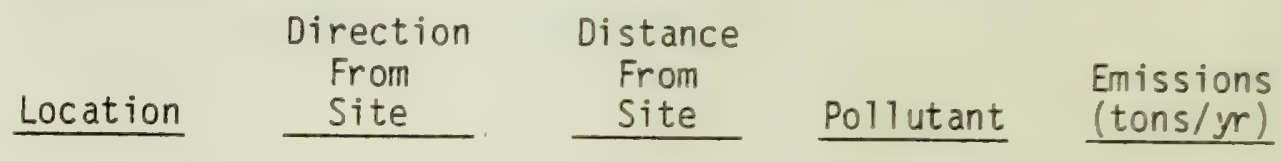

None

2. Mobile Sources that may Influence the Site:

Names of Roadways:

Churchill

Jefferson

Type:

Arterial Street

or Highway

Local Street

or Road

Distance of Roadway from Site (ft) 80

500

Composition of Roadway

Asphalt

Asphalt

Number of Traffic Lanes

Average Daily Traffic

2000

9900

Average Vehicle Speed (mph)

One or Two Way Traffic

Two

Two

Number of Parking Lanes

One

None

Are Parking Lanes Used

For Traffic Part of Day?

No

N/A

Is Dust Visibility Retained?

№

Yes

Does Roadway Have Curb

Yes

No 
3. Area Sources that may Influence the Site:

\begin{tabular}{lcccc} 
Type of Source & From Site & & $\begin{array}{c}\text { Distance From } \\
\text { From Site }\end{array}$ & Pollutant \\
\cline { 2 - 3 } Agricultural & SW & & 0.8 mile & TSP \\
Construction (1ocal) & SSW & & $100-300$ feet & TSP
\end{tabular}

D. Topograhy/Obstructions

1. General Characteristics Over a 2 Mile Radius From the Site: Smooth

2. Topographic Features that Influence the Site: (types - hills, valleys, depressions, bodies of water, ridges, cliffs)

$\begin{array}{llll}\text { Type } & \text { Size } & \begin{array}{c}\text { Direction } \\ \text { From Site }\end{array} & \begin{array}{c}\text { Distance } \\ \text { From Site }\end{array} \\ \text { Ravine } & 50 \mathrm{ft} \text {. depth } & \text { SSW } & 350 \mathrm{ft} \text { to center }\end{array}$

3. Obstructions to wind Flow

(types - buildings, trees ridges, cliffs)

\begin{tabular}{llll} 
Type & Size & $\begin{array}{l}\text { Direction } \\
\text { From Site }\end{array}$ & Distance \\
\hline
\end{tabular}

None

4. Comments

IEPA building is located partiy down the ravine note above. The top of the building on which the hi vol is located is probably only 25 feet above terrain to the north.

E. Distance to the nearest National Weather Service (NWS) Site.

Here are the distances and directions to the nearest NWS observing site from the inhalable particulate monitor in Springfield. Distances are in statute miles; directions are degrees from true north.

Site Address

IEPA BIdg.

2200 Churchi11 Road
Nearest NWS Site

Capital Airport/Springfield (SPI)
Directions \&

Distance to NWS

$020^{\circ}$ at $3.2 \mathrm{mi}$. (NNW) 
Springfield-IEPA - S

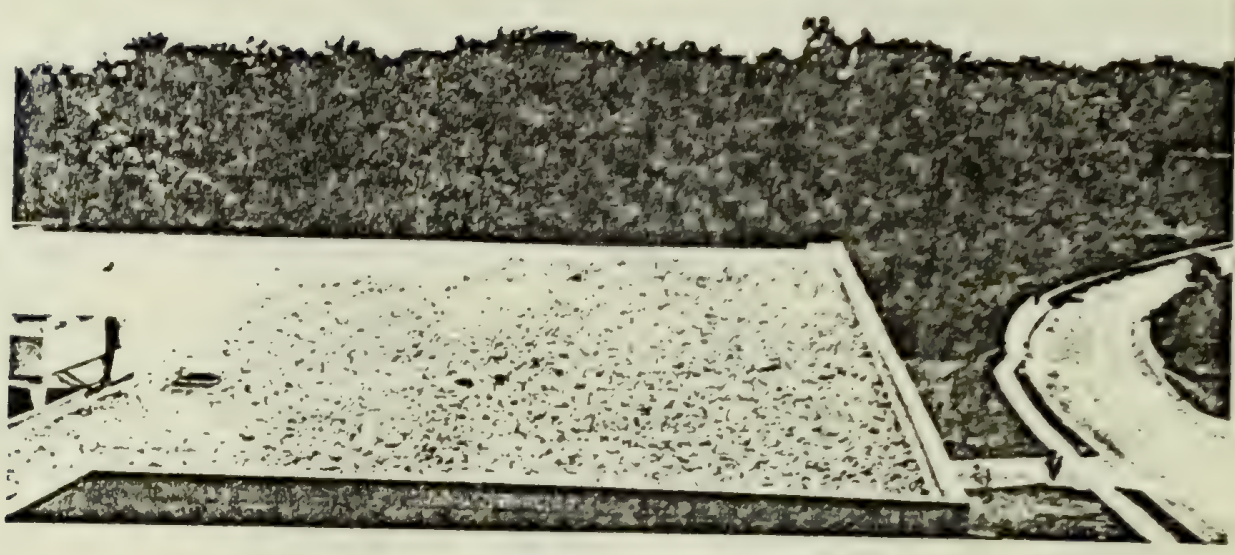

Springfield IEPA - South

Springfield-IEPA - W

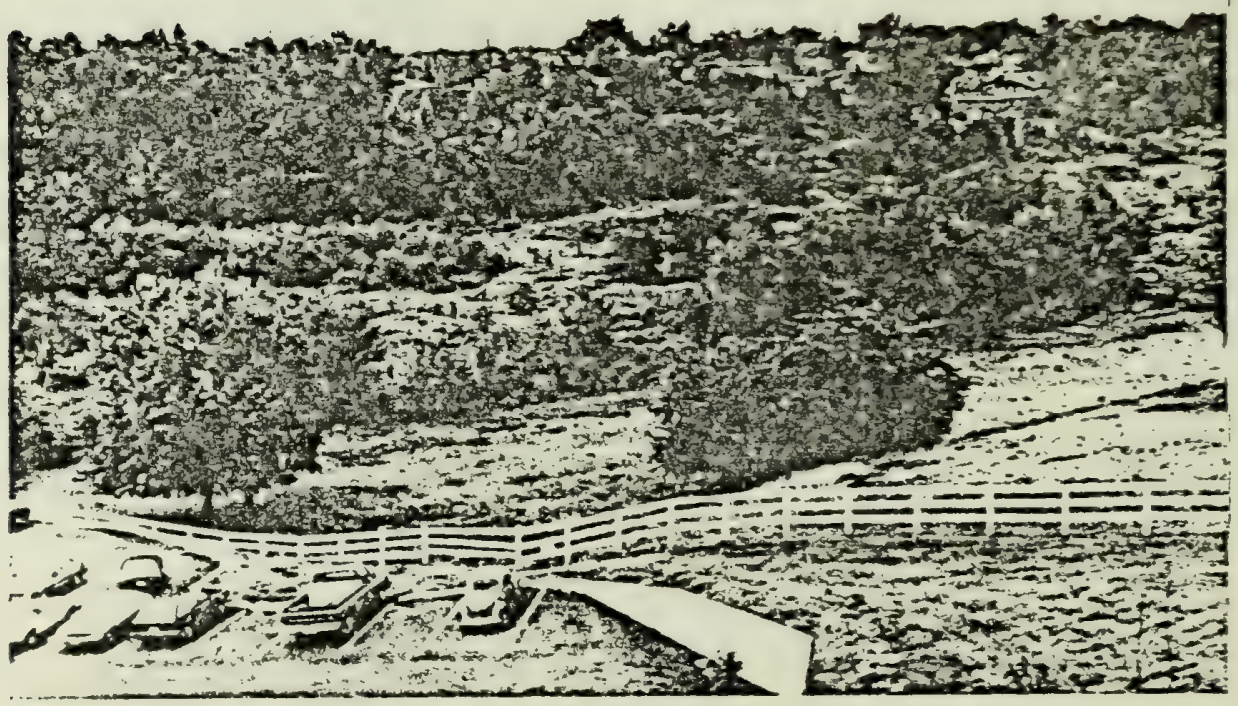

Springfield IEPA - West 


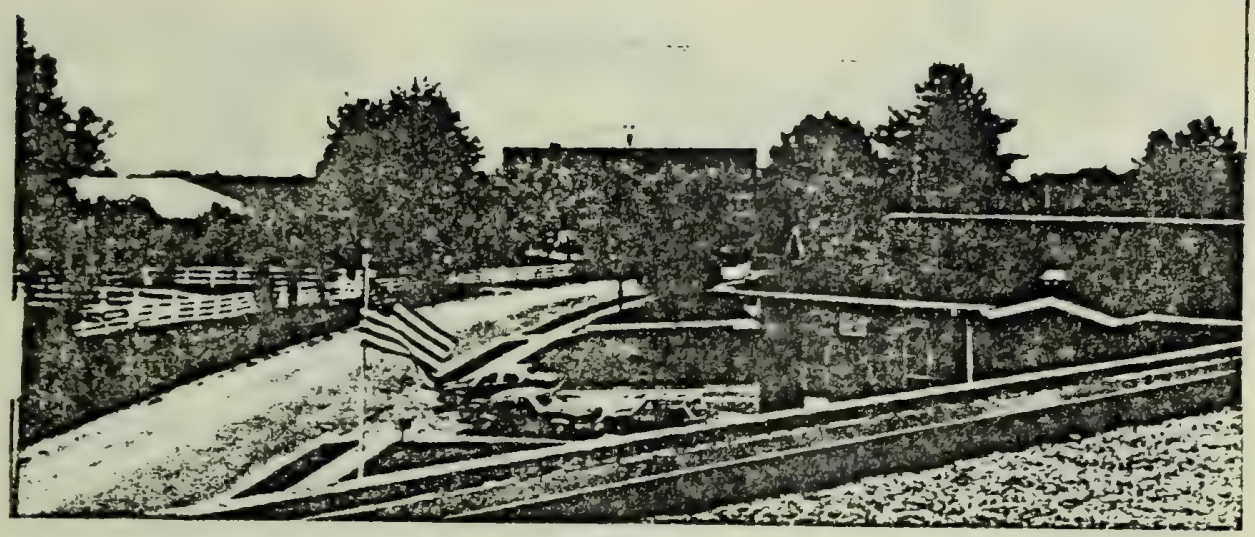

Springfield IEPA - North
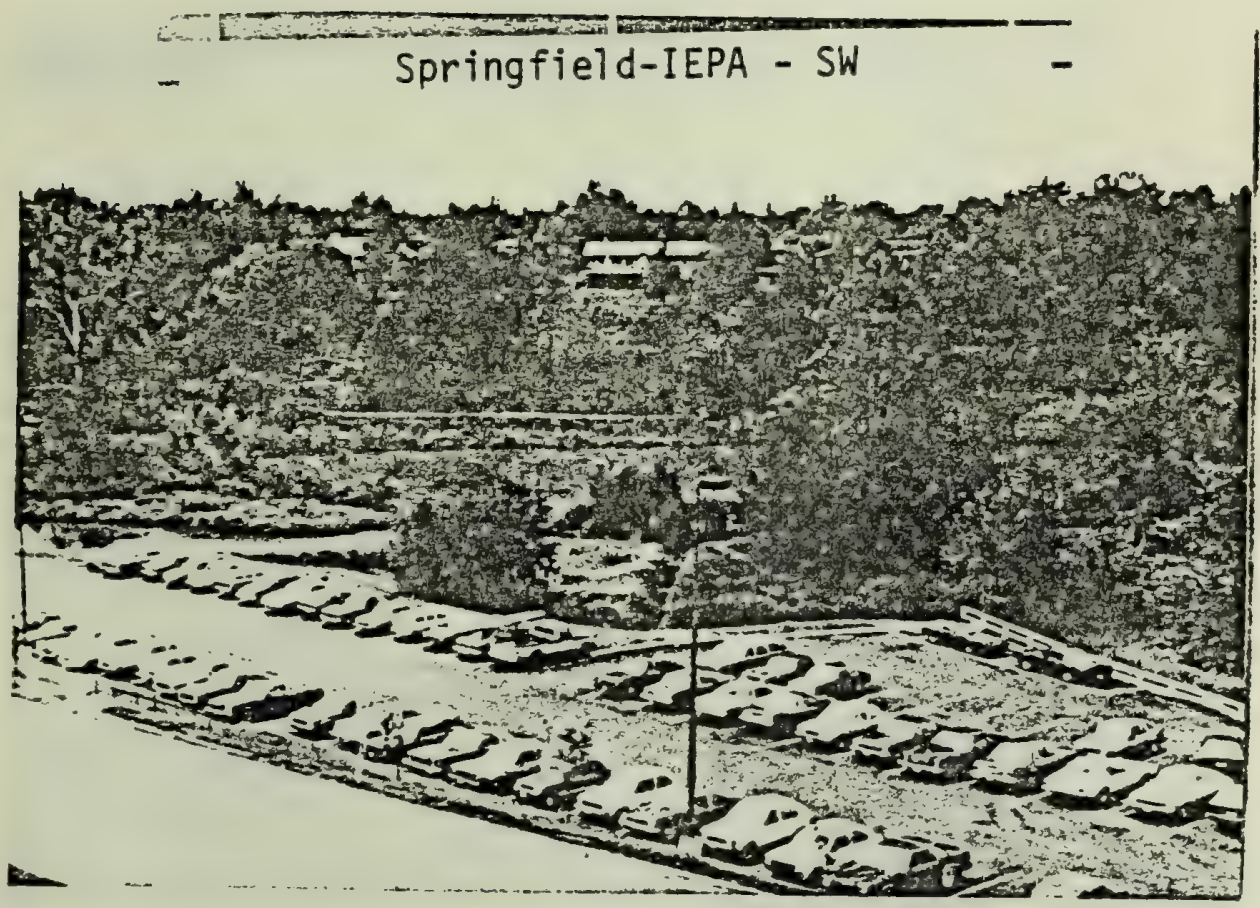

Springfield IEPA - South West 


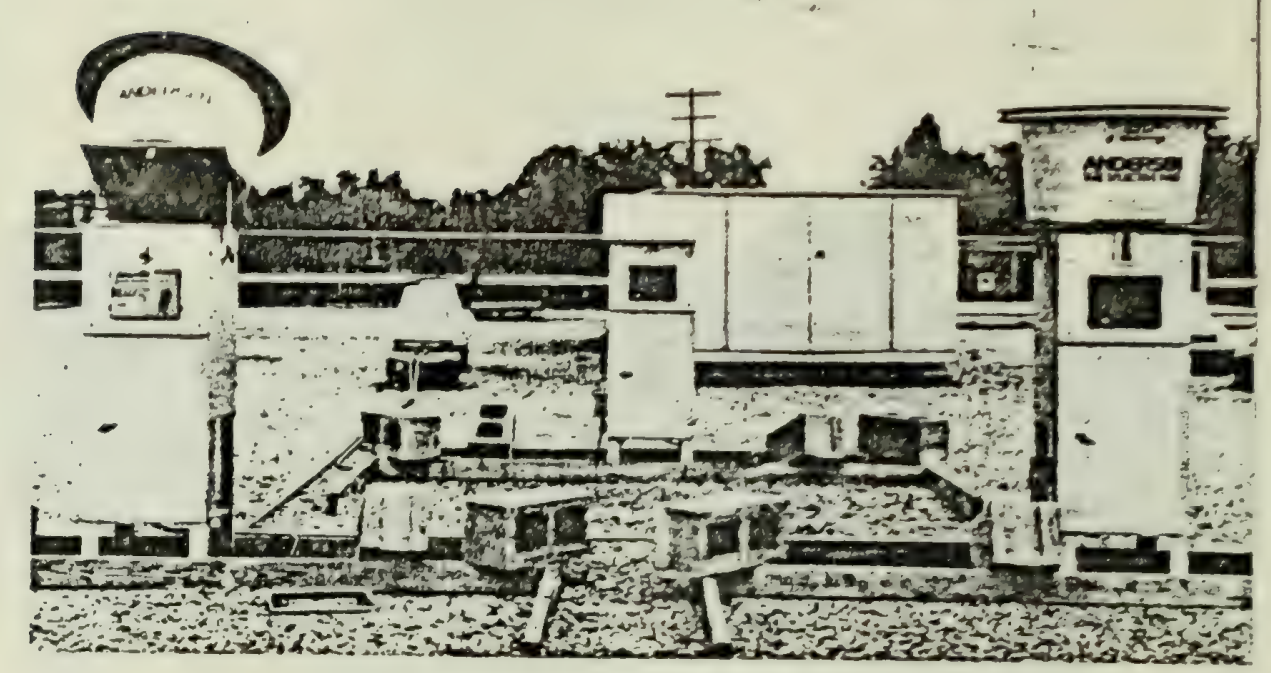

Springfield IEPA - Roof
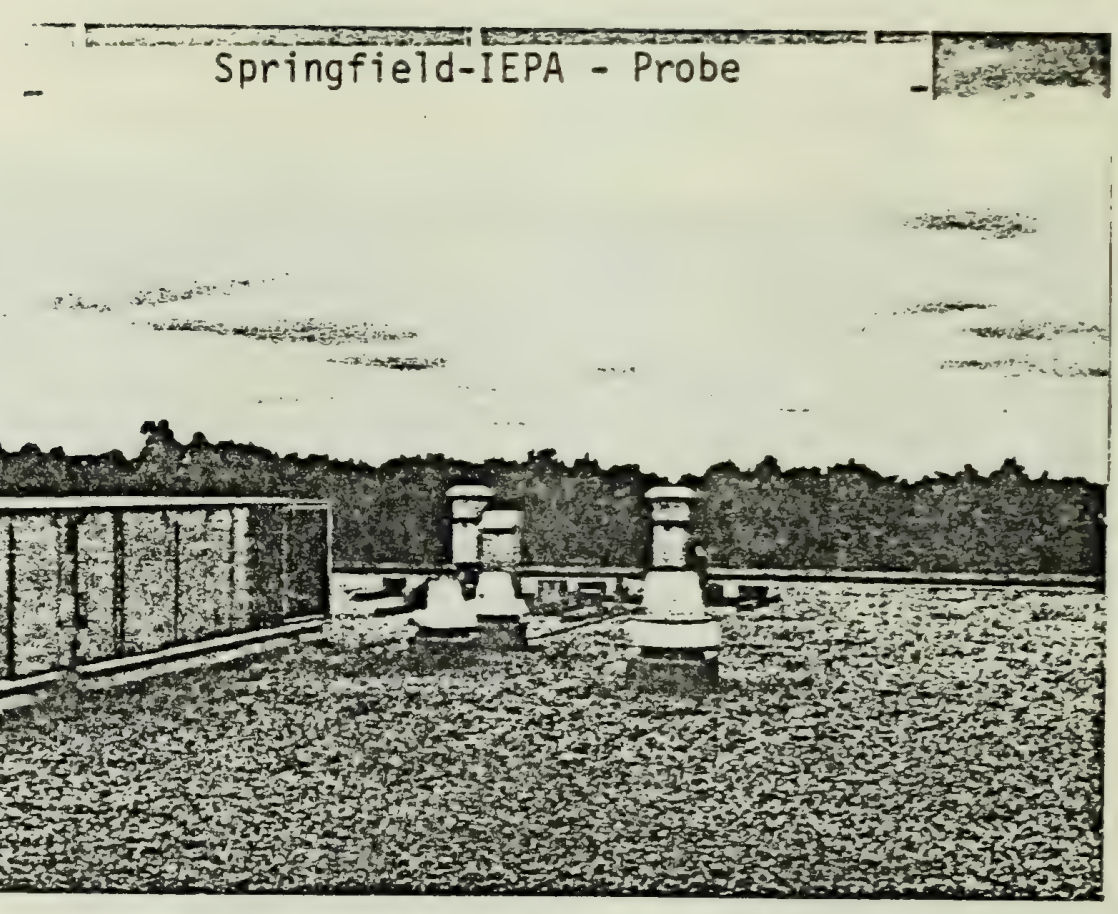

Springfield IEPA - Roof 


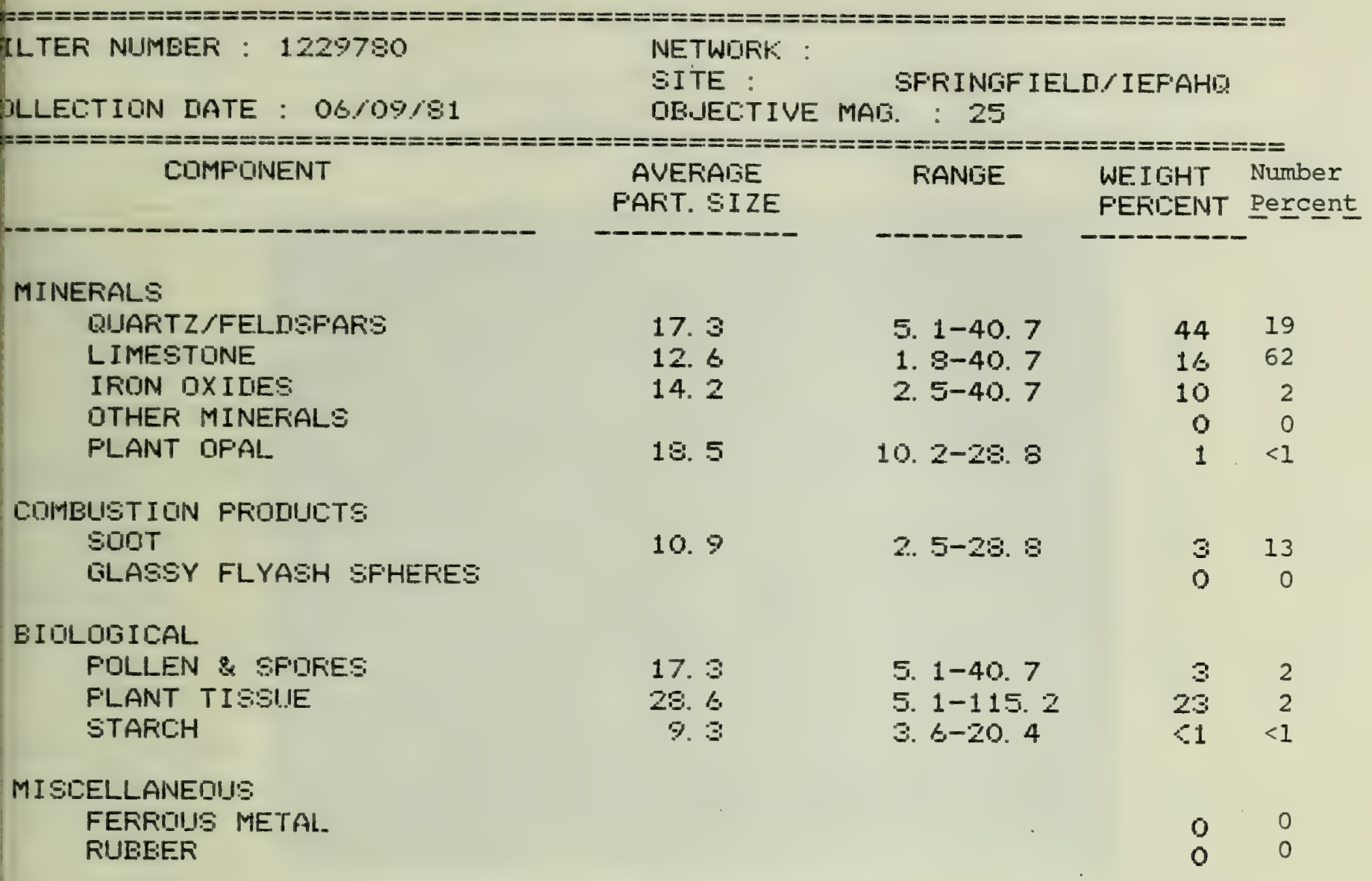

FAFTICLE SIZE DISTRIEUTIONN

\begin{tabular}{|c|c|c|c|}
\hline SIZE & RANISE (UIM) & $\begin{array}{l}\text { NUMMEER } \\
\text { FEFILENT }\end{array}$ & $\begin{array}{l}\text { WEIGHT } \\
\text { FERIOENT }\end{array}$ \\
\hline 1. & $3-1.5$ & 0 & 0 \\
\hline 1. & $8-2.5$ & $<1$ & $<1$ \\
\hline 2. & $5-3 t$ & 50 & $<1$ \\
\hline 3. & $6-5.1$ & 7 & $<1$ \\
\hline 5. & $1-7.2$ & 11 & $<1$ \\
\hline 7. & $2-10.2$ & 12 & 3 \\
\hline 10. & $2-14.4$ & 8 & 6 \\
\hline 14. & $4-20.4$ & 6 & 11 \\
\hline 20. & $4-25.8$ & 4 & 22 \\
\hline 28. & $8-40.7$ & 2 & 35 \\
\hline 40. & $7-57.6$ & 0 & 0 \\
\hline 57. & $\Leftrightarrow-81.5$ & 0 & 0 \\
\hline 81. & $5-115.2$ & $<1$ & 22. \\
\hline 115. & $2-1 \leqslant 2.9$ & 0 & 0 \\
\hline $1 \Leftrightarrow 2$. & $9-230.4$ & 0 & 0 \\
\hline & 3230.4 & 0 & 0 \\
\hline
\end{tabular}


Total Suspended Particulate ug/m $\mathrm{m}^{3}$

$\begin{array}{cclc}\text { Particulate: Minerals } & 36.2 & \text { Combustion } & 1.5 \\ \text { Biological } 13.3 & \text { Other } & - \\ \text { Low temperature ashing, } \% \text { loss } & & 37.0\end{array}$
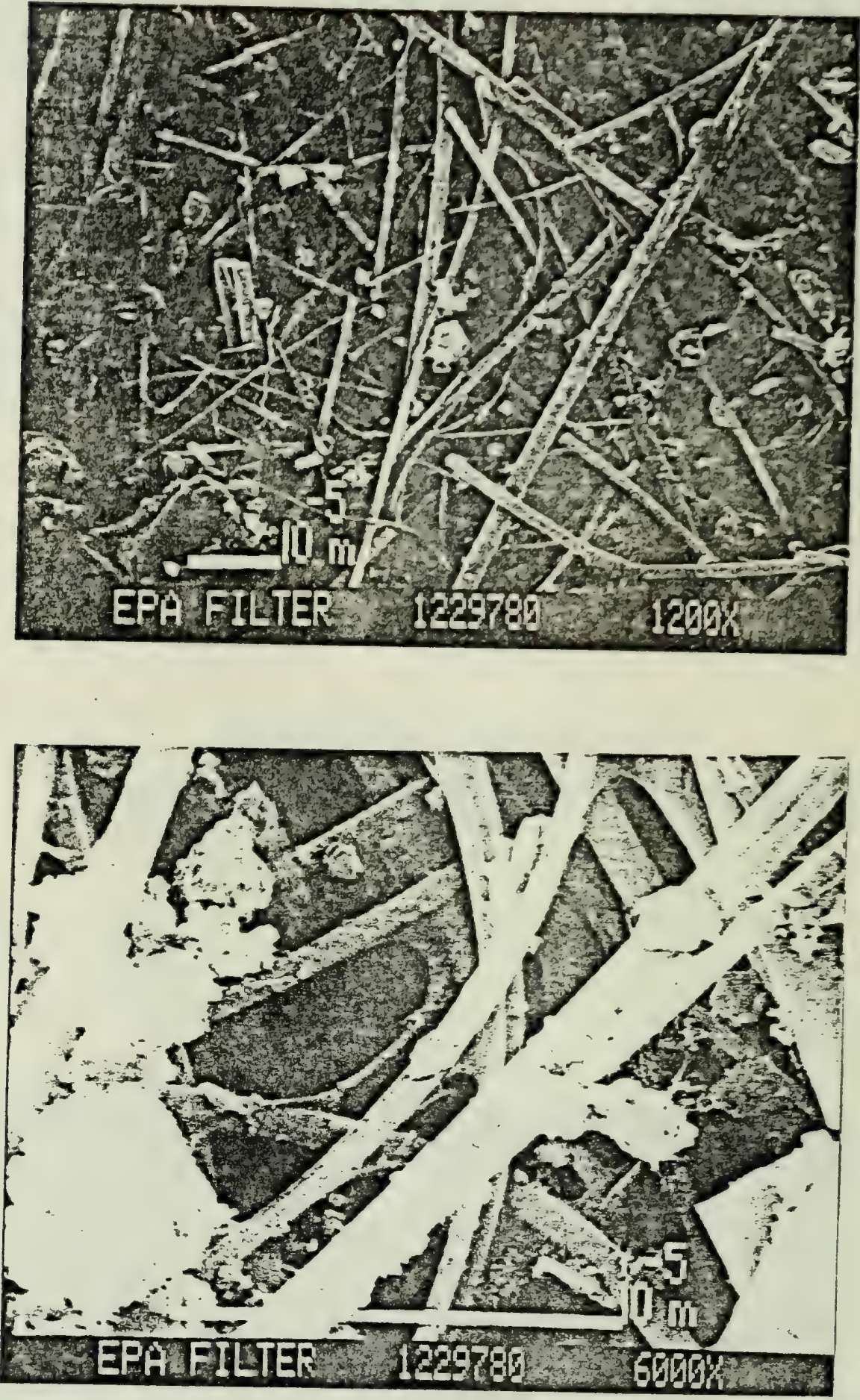
LECTION DATE: O6/09/81 NETWORK :

SITE : GFRINGF IELDIIEPAHD

DE.AECTIVE MAB. : 25

\section{CMMPONENT}

AVERAIIE

PAFT. SIZE
RANISE.

3. $8-28.8$

2. $5-40.7$

2. $5-14.4$

IFION OXIDES

OTHEF MINEFALS
12. 4

7. 6

12. 3

7. 9

13. 8

12. 8

12. 3

5. $1-28.8$

7. $2-20.4$

7. $2-20.4$

3. $6-14.4$

$\begin{array}{rr}3 & 13 \\ 0 & 0\end{array}$

WEIGHT Number

PERICENT Fercent

3 IOLOGICAL

FOLILEN \& SFORES

FLANT TISSUIE

STAFICH

\section{I SCELLLANEOUIS}

FEFRIOUS METAL.

RUEBER

14. 9

10. $2-20.4$

0

2

$\begin{array}{rr}37 & 22 \\ 44 & 56 \\ 1 & 1 \\ 0 & 0\end{array}$

FAFTIOLE SIZE DISTRIEIITIOIN

\begin{tabular}{cc} 
SIZE FANISE (UM) & NLIMEER \\
& FEFCENT \\
\hline $1.3-1.8$ & 0 \\
$1.8-2.5$ & 0 \\
$2.5-3.6$ & 28 \\
$3.6-5.1$ & 20 \\
$5.1-7.2$ & 15 \\
$7.2-10.2$ & 22 \\
$10.2-14.4$ & 11 \\
$14.4-20.4$ & 4 \\
$20.4-29.6$ & 01 \\
$28.8-40.7$ & 61 \\
$40.7-57.6$ & 0 \\
$57.6-51.5$ & 0 \\
$81.5-115.2$ & 0 \\
$115.2-162.9$ & 0 \\
$162.9-230.4$ & 0 \\
3230.4 & 0
\end{tabular}

WEI IBHT

F'EFILENT

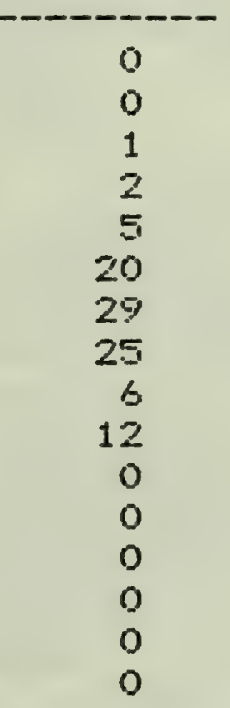


Inhalable Particulate

$$
\mu g / m^{3}
$$

$\begin{array}{rllr}\text { Particulate: Minerals } 32.8 & \text { Combustion } & 1.2 \\ \text { Biological } & 10.4 & \text { Other } & 0.8 \\ \text { Low temperature ashing, } \% \text { loss } & & 30.0\end{array}$
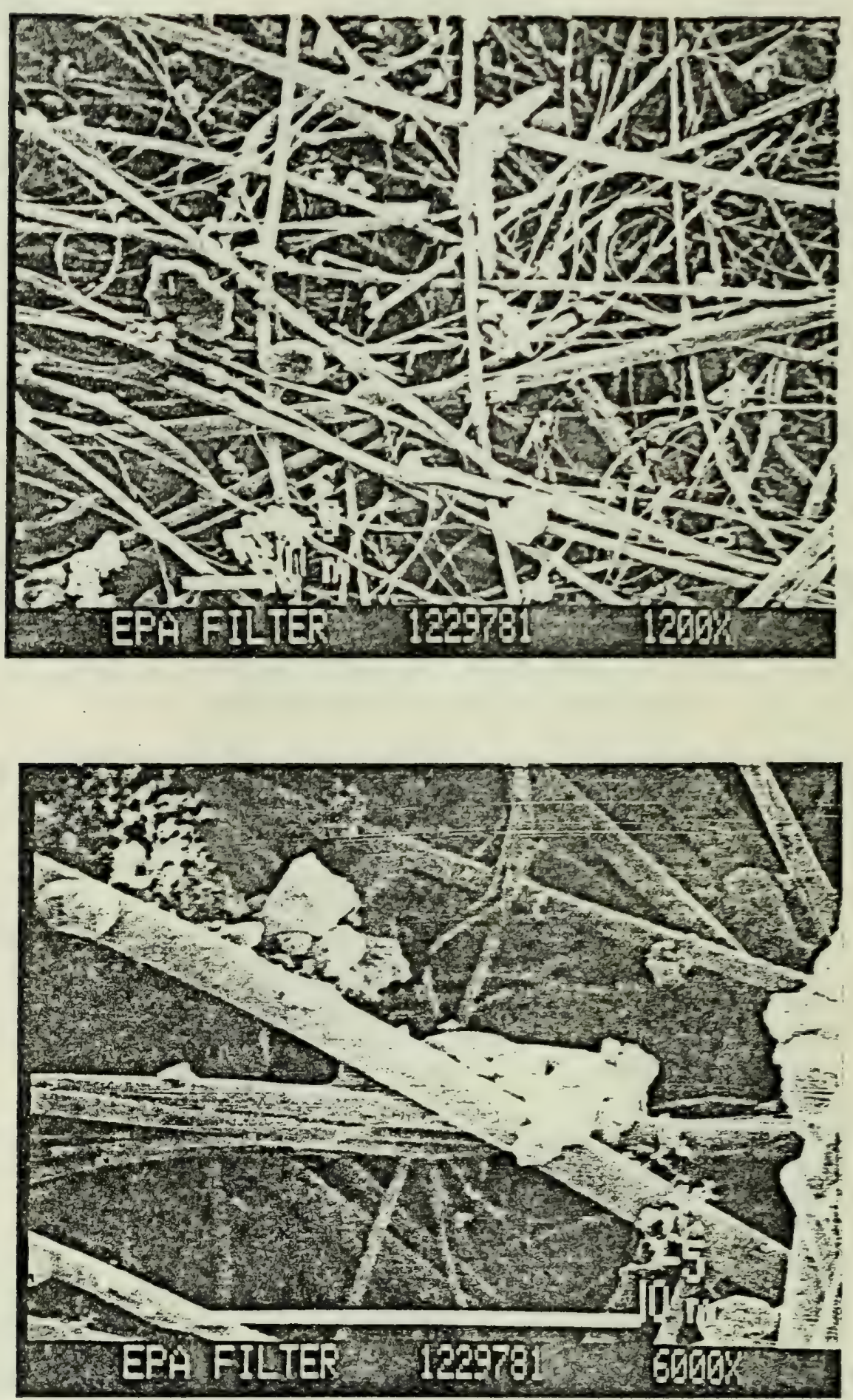

Particulate:
55.2
Combustion
10.1
Biological 26.7 Other
Low temperature ashing, \% loss
24.2
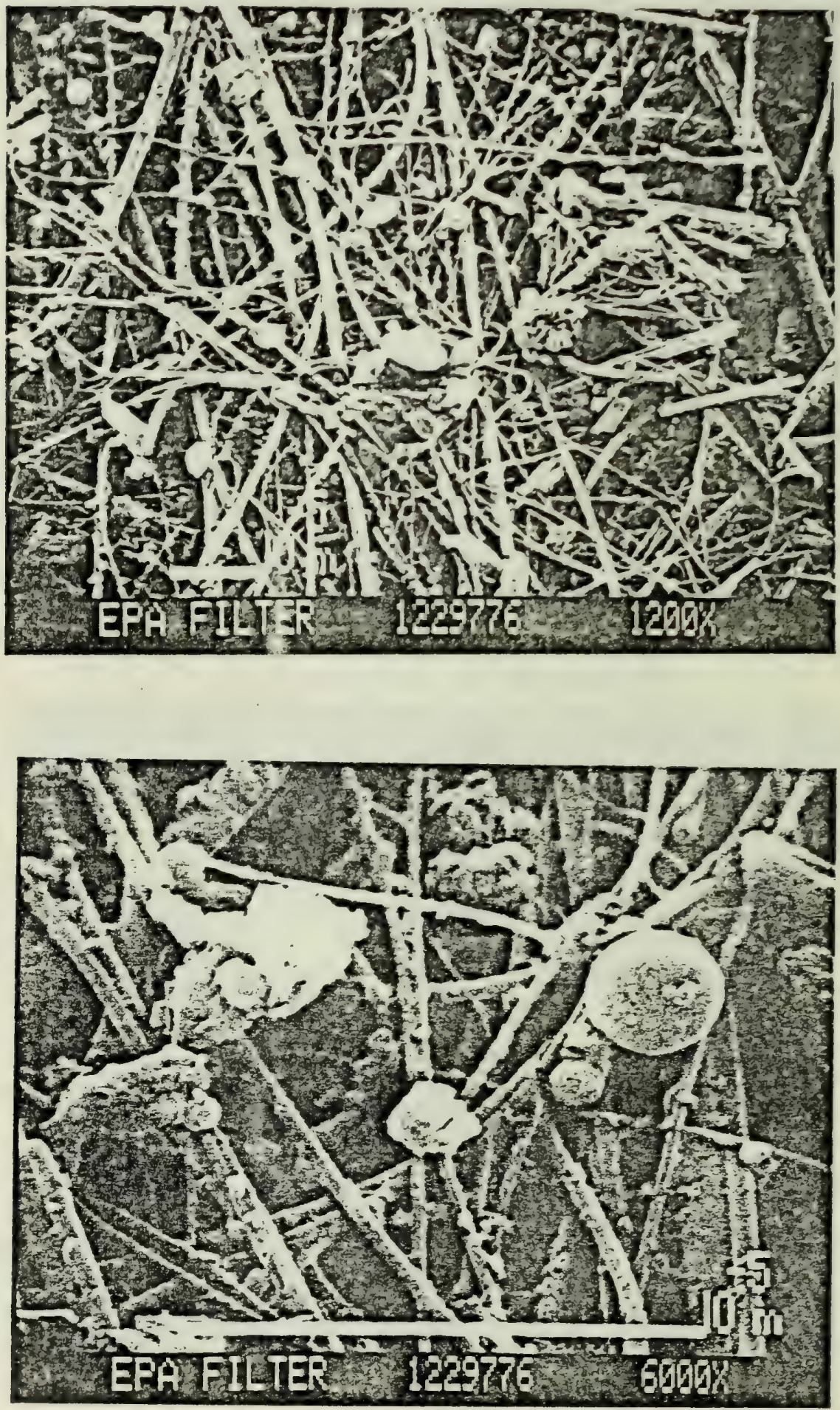


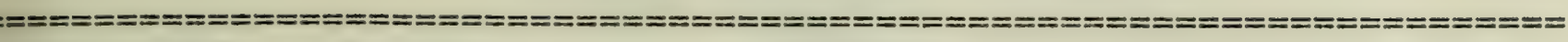

L.TER NLIMEER : 1277250

M.LEETION DATE : 07/03/81
NETWIDK :

SITE : $\quad$ SFRINLIF IELD/IEFAHD
COMMPINENT
DE.JECTIVE MAIS. : 25

\begin{tabular}{|c|c|c|c|c|}
\hline C.OMPIINENT & $\begin{array}{l}\text { AVERAGE } \\
\text { PART. SIZE }\end{array}$ & RANIBE & $\begin{array}{l}\text { WE JLIHT } \\
\text { F'ERIENT }\end{array}$ & $\begin{array}{l}\text { Number } \\
\text { Percent }\end{array}$ \\
\hline \multicolumn{5}{|l|}{ MINERALS } \\
\hline RUARTZ/FEI_DSFAR: & 9.8 & 3. 6.20 .4 & 51 & 40 \\
\hline LIMESTONE & 9.8 & $3.6-20.4$ & 5 & 11 \\
\hline IFUUN OXIDES & 7. 2 & 1. $3-14.4$ & 2 & 2 \\
\hline OTHER MINERALS & & & 0 & 0 \\
\hline \multicolumn{5}{|l|}{ COMEUUSTIUN FRODIILTS } \\
\hline SODT & 10.9 & 2. $5-28.8$ & 19 & 36 \\
\hline GI_ASSY FLYASH SFHEFES & & & 0 & 0 \\
\hline \multicolumn{5}{|l|}{ BIOL_OBICAL } \\
\hline FOLLEN \& SPLIFES & 19. 7 & 5. $1-57.8$ & 15 & 5 \\
\hline FLANT TISSLIE & 14. 2 & 5. $1-29.8$ & 3 & 3 \\
\hline STARICH & 14. 2 & 5. $1-28.8$ & 5 & 3 \\
\hline \multicolumn{5}{|l|}{ MISCELLANEGIIS: } \\
\hline FEFFIUIIS METAL & & & 0 & 0 \\
\hline RUEBEF & & & 0 & 0 \\
\hline
\end{tabular}

\section{FARTICILE EIZE DISTFIEUITION}

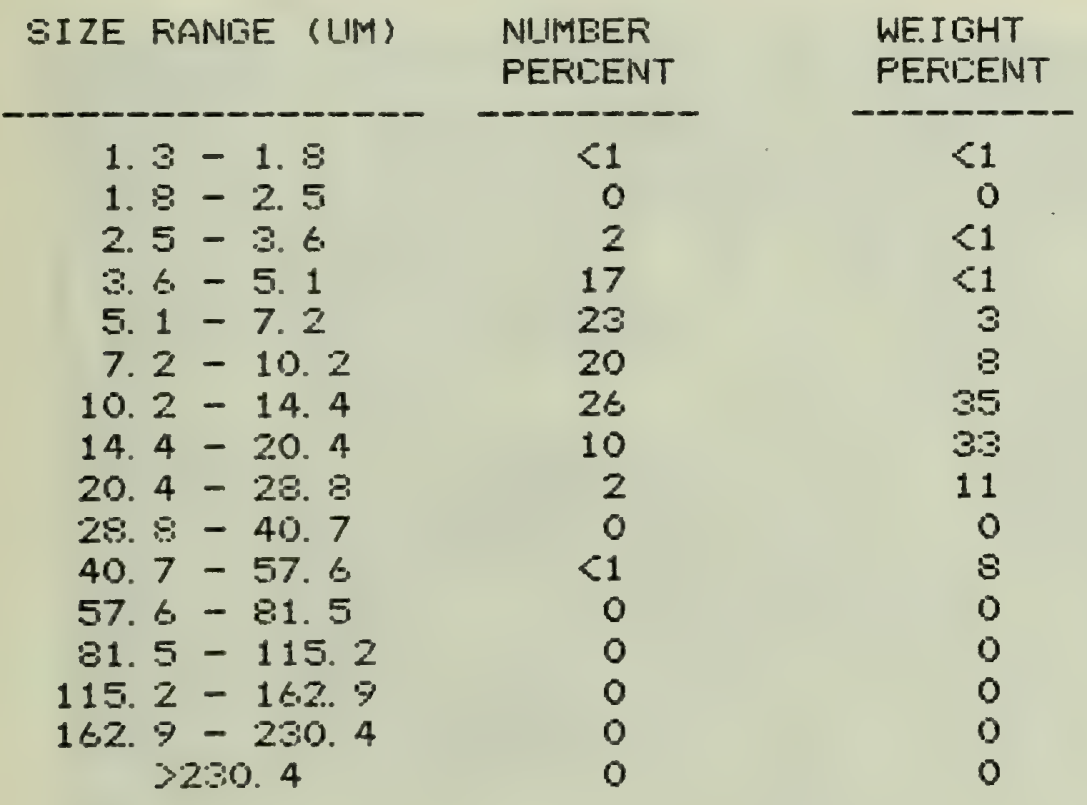


Inhalable Particulate $\quad \mu g / \mathrm{m}^{3}$

$\begin{array}{cclc}\text { Particulate: Minerals } & 50.5 & \text { Combustion } & 16.5 \\ \text { Biological } 20 & \text { Other } & - \\ \text { Low temperature ashing, } \% \text { loss } & & 20.3\end{array}$
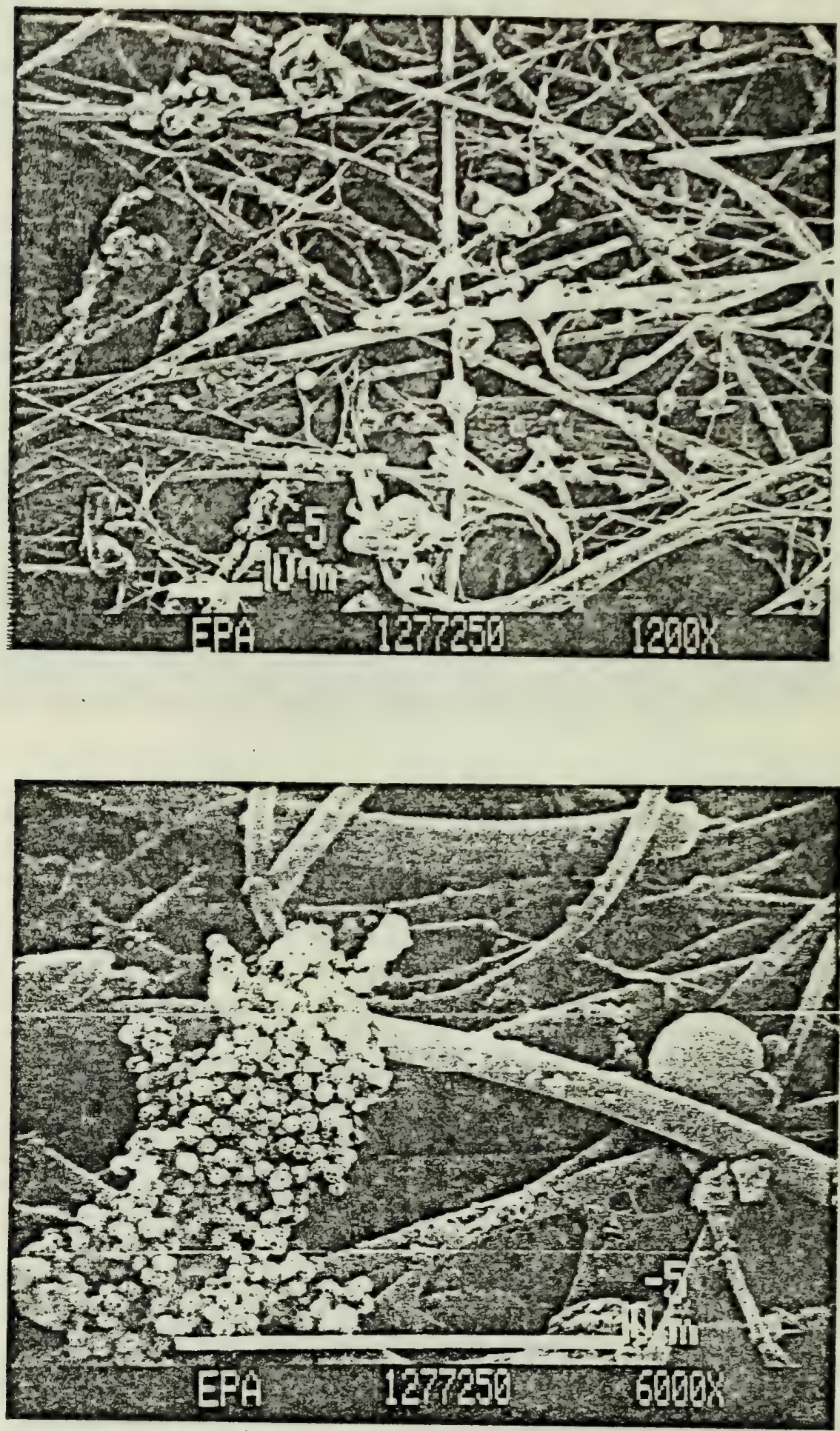


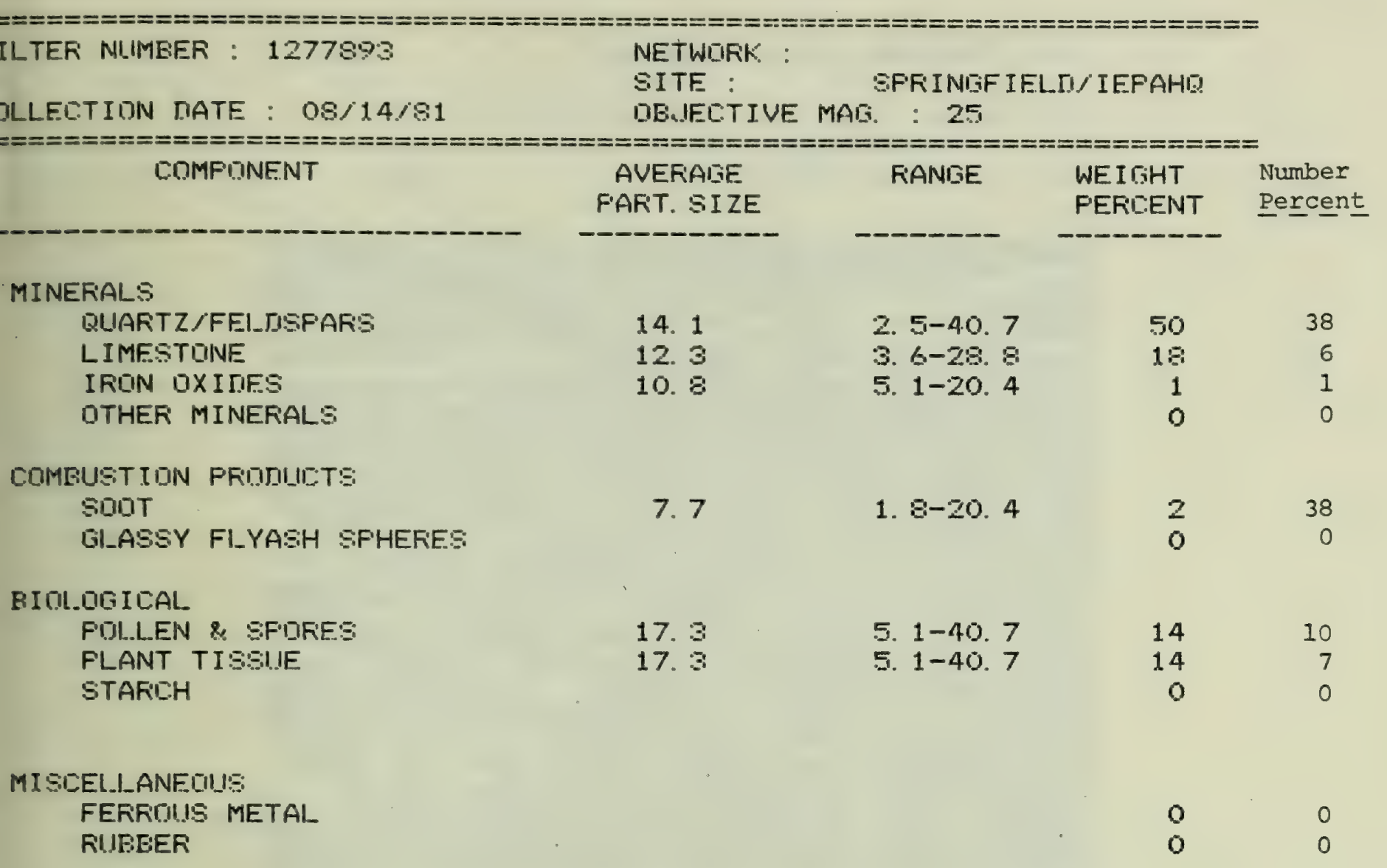

FAFTILLE SIZE DISTFIEILITION

\begin{tabular}{|c|c|c|c|}
\hline SIZE & RAINISE (INM) & $\begin{array}{l}\text { NIIMEER } \\
\text { FEFICENT }\end{array}$ & $\begin{array}{l}\text { WEIDHHT } \\
\text { F'ERILENT }\end{array}$ \\
\hline & $3-1.8$ & 0 & 0 \\
\hline & $8-2.5$ & 14 & $<1$ \\
\hline & $5-36$ & 26 & $<1$ \\
\hline & $b-5.1$ & 13 & $<1$ \\
\hline & $1-7.2$ & 14 & 2 \\
\hline & $z-10.2$ & 8 & 2 \\
\hline 10. & $2-14.4$ & 6 & 4 \\
\hline 14. & $4-20.4$ & 7 & 17 \\
\hline 20. & $4-29.8$ & 5 & 3.7 \\
\hline 28 & $B-40.7$ & 2 & 41 \\
\hline 40. & $7-57.6$ & 0 & 0 \\
\hline 57. & $b-81.5$ & 0 & 0 \\
\hline 81. & $5-115.2$ & 0 & 0 \\
\hline 115. & $2-162.9$ & 0 & 0 \\
\hline \multirow[t]{2}{*}{162} & $9-230.4$ & 0 & 0 \\
\hline & 2230.4 & 0 & 0 \\
\hline
\end{tabular}


Total Suspended Particulate $\mu g / \mathrm{m}^{3}$

Particulate: Minerals 59.3 Combustion 1.7

Low temperature ashing, \% loss

29.9
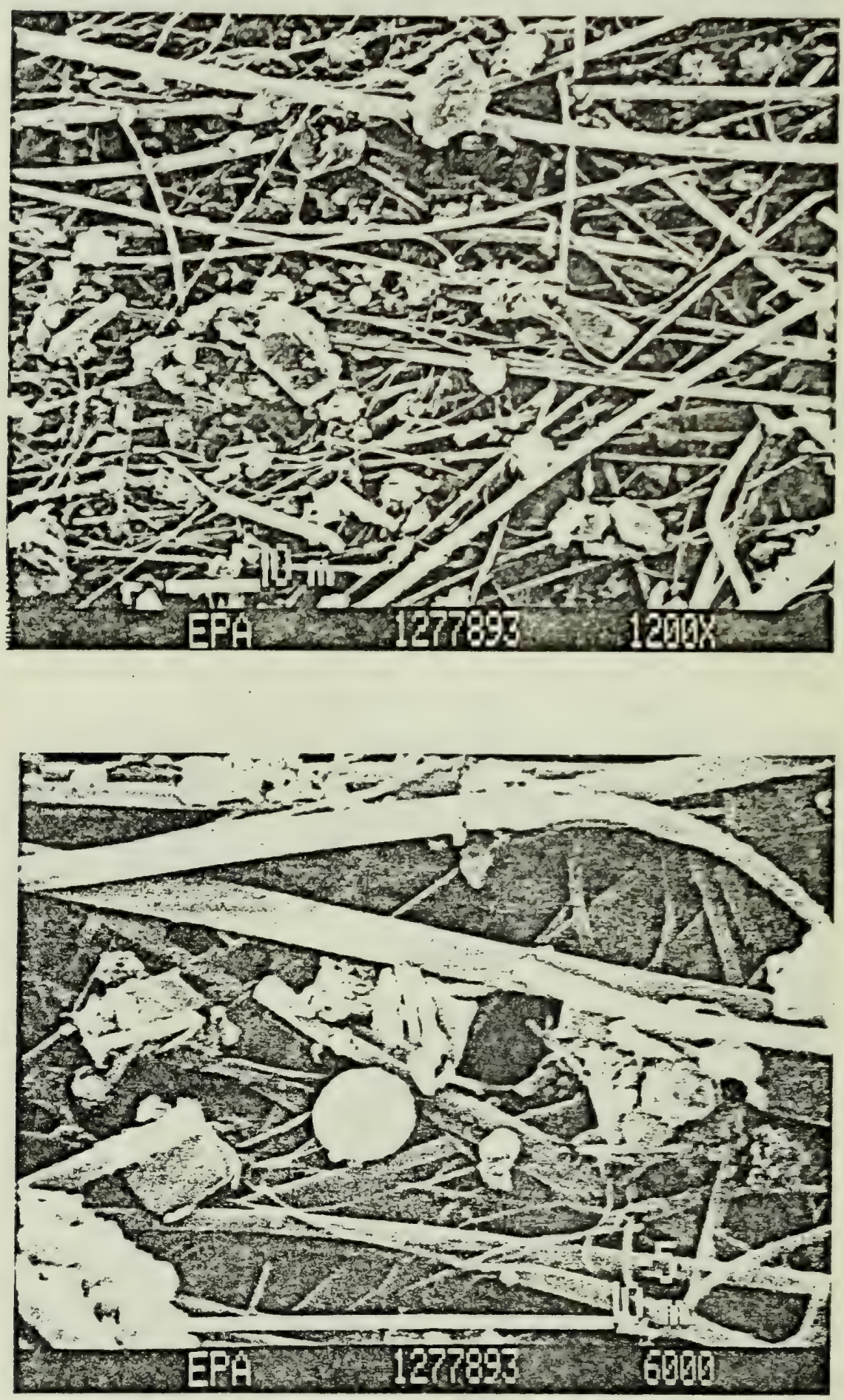


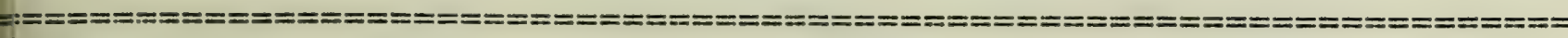

1.TER NLIMEER : 1277994

NETWIIRK :

SITE :

SFFI INGF IELIJ/IEFAHE!

CLEETION DATE : 0\%/14/81

DE.IELTIVE MAG. : 25

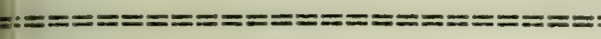
COMFIINENT

AVERIATE

FAFIT. SIZE

13.8

8. 7

6. 4

9.5

2. $5-20.4$

5. $1-28.8$

2. $5-20.4$

3. $6-10.2$

OTHER MINEFIALS

:UMEIJSTIOIN FRUIIILTS

SOOT

GIIASSY FLYASH SFHEFEE:

TIOLOGICAL

FILLEN \& SFIIFE:

PILANT TISEIIE

STARC.H
17. 3

17. 3

17. 4
FANISE

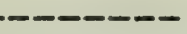

5. $1-40.7$

5. $1-40.7$

14. $4-20.4$
WEITHT

FERTCENT
Number Percent

\section{ISICELI_ANEOUIS}

FEFROLSS METAL

FIUEAEF

43

9

$<1$

0

3

0

7

13

$<1$

\section{FARTIDLE SIZE DISTFIEUTIDIN}

\begin{tabular}{cc} 
SIZE FANIE (IIM) & NIIMEEF \\
FEFIEENT \\
\hline $1.3-1.8$ & 0 \\
$1.8-2.5$ & 0 \\
$2.5-3.6$ & 39 \\
$3.6-5.1$ & 7 \\
$5.1-7.2$ & 24 \\
$7.2-10.2$ & 11 \\
$10.2-14.4$ & 9 \\
$14.4-20.4$ & 9 \\
$20.4-28.8$ & 6 \\
$28.8-40.7$ & 0 \\
$40.7-57.6$ & 0 \\
$57.6-51.5$ & 0 \\
$91.5-115.2$ & 0 \\
$115.2-162.9$ & 0 \\
$162.9-230.4$ & 0 \\
$32: 30.4$ & 0
\end{tabular}

WE IIIHT

FEFILENT

0
0
$<1$
$<1$
4
3
3
22
47
14
0
0
0
0
0
0




\begin{tabular}{lllc} 
Inhalable Particulate & \multicolumn{2}{c}{$74 / \mathrm{m}^{3}$} & \\
Particulate: Minerals & 38.5 & Combustion & 2.2 \\
Biological & 32.6 & Other & - \\
Low temperature ashing, $\%$ loss & & 24.0
\end{tabular}
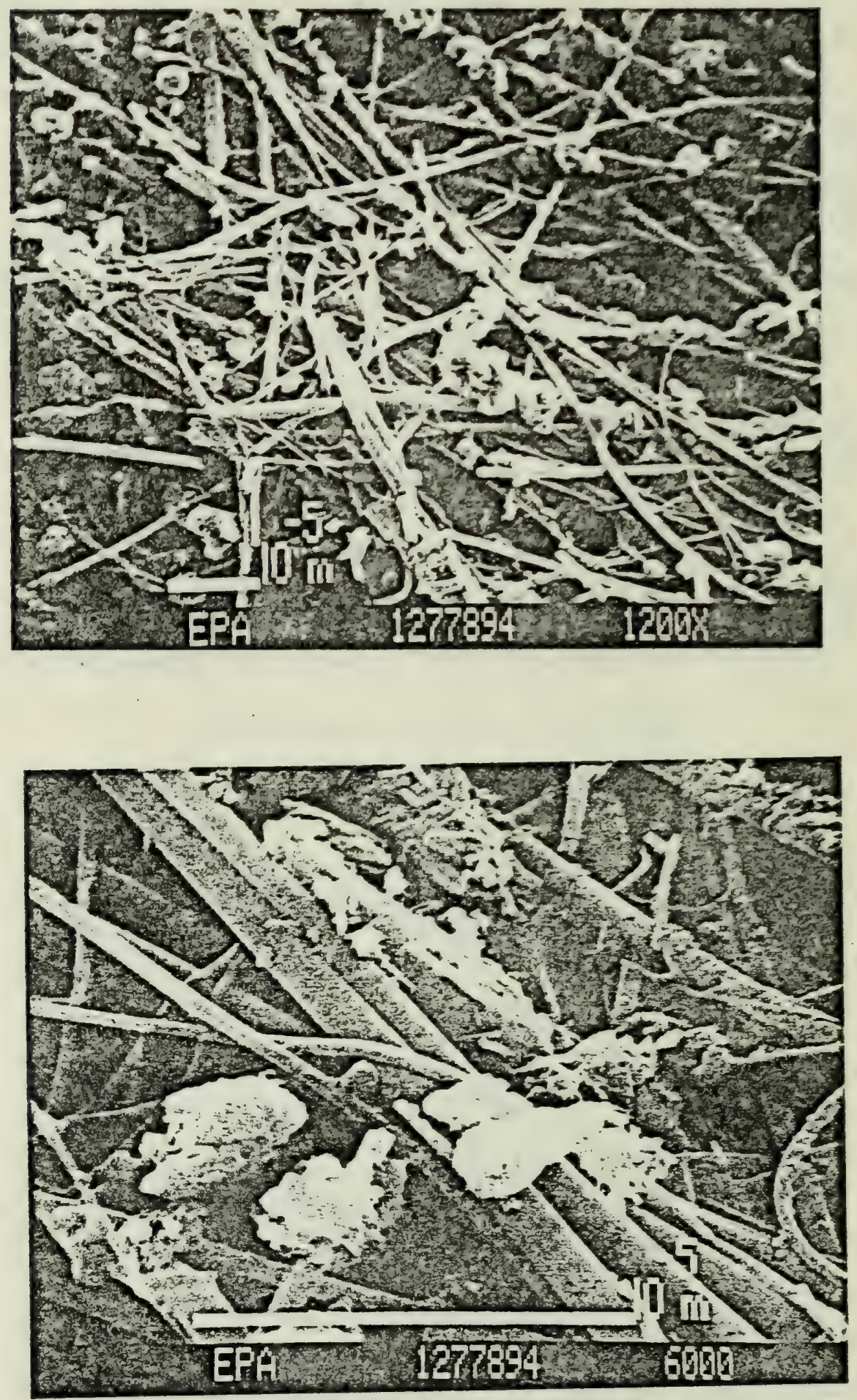
A. Site Identification

1. City: Joliet

2. Site Name and Address: Pershing School, Midland and Campbell

3. County: Will Township: Joliet

4. USGS Topographical Map Name and Scale: Joliet, 1:24000

5. Site Elevation (Feet): 653

B. Site Classification/Representativeness

1. Dominating Influence on Site: Residential

C. Source Impact

1. Stationary Sources that may Influence Site:

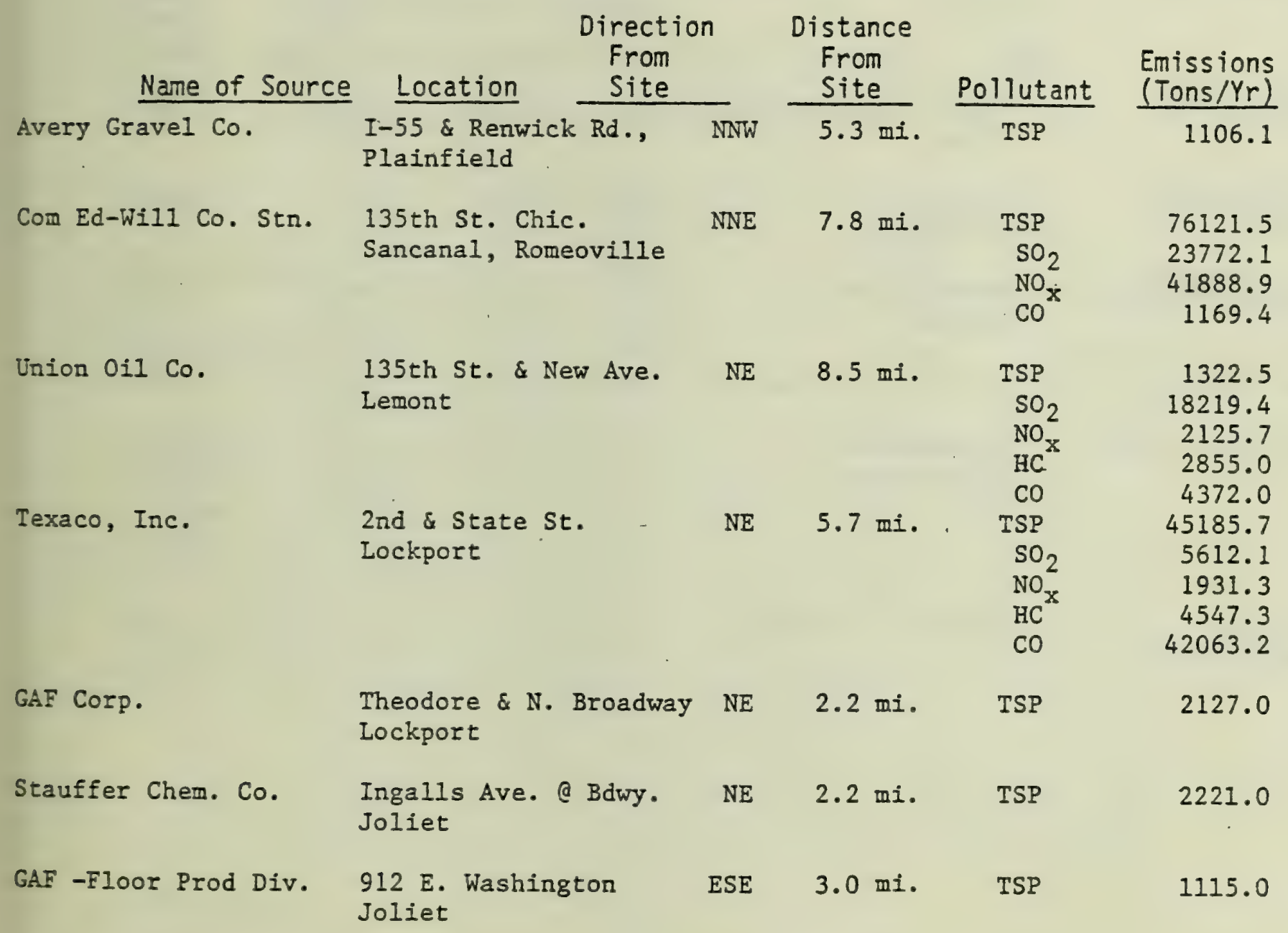




\begin{tabular}{|c|c|c|c|c|c|}
\hline Vulcan Materials Co. & $\begin{array}{l}\text { Mills Rd \& Rt } 53 \\
\text { Joliet }\end{array}$ & $S E$ & $2.5 \mathrm{mi}$. & TSP & 2413.4 \\
\hline Crown Trygg Corp. & $\begin{array}{l}1127 \text { s. Chicago St. } \\
\text { Joliet }\end{array}$ & $S E$ & $2.5 \mathrm{~m} 1$ & TSP & 2029.4 \\
\hline Ceramic Powders & $\begin{array}{l}200 \text { Moen Ave. } \\
\text { Rockdale }\end{array}$ & SSE & $1.6 \mathrm{ml}$. & TSP & 1729.7 \\
\hline General Refractories & $\begin{array}{l}\text { Larkin \& Moen } \\
\text { Rockdale }\end{array}$ & $\mathrm{s}$ & $1.6 \mathrm{mi}$ & TSP & 7536.7 \\
\hline Delta Const. Inc. & $\begin{array}{l}1711 \text { Brandon Rd. } \\
\text { Joliet }\end{array}$ & $\left.\right|^{\text {SSE }}$ & 3.0. mi. & TSP & 83275.5 \\
\hline $\begin{array}{l}\text { Stepan Chem Co. } \\
\text {-Ind Chem. Div. }\end{array}$ & RR1, Elwood & SSW & $6.9 \mathrm{mi}$ & $\begin{array}{r}\text { TSP } \\
\text { HC } \\
\text { CO }\end{array}$ & $\begin{array}{l}4069.4 \\
4438.7 \\
6303.7\end{array}$ \\
\hline Caterpillar & $\begin{array}{l}\text { Box } 504 \text { Channahon } \\
\text { Jollet }\end{array}$ & SW & $2.8 \mathrm{mi}$ & $\begin{array}{l}\mathrm{TSP} \\
\mathrm{SO}_{2}\end{array}$ & $\begin{array}{l}3056.0 \\
1091.0\end{array}$ \\
\hline $\begin{array}{l}\text { Mobil Joliet } \\
\text {-Refinery Corp }\end{array}$ & $\begin{array}{l}\text { I-55 \& Smith Bridge Rd. } \\
\text { Joliet }\end{array}$ & SW & $8.4 \mathrm{mi}$ & $\begin{array}{l}\mathrm{TSP} \\
\mathrm{SO}_{2} \\
\mathrm{NO}_{\mathrm{x}} \\
\mathrm{CO}\end{array}$ & $\begin{array}{r}4089 \\
27608 \\
3308 \\
42093\end{array}$ \\
\hline Mobil Chem Co. & $\begin{array}{l}\text { I-55 \& Old Arsenal Rd. } \\
\text { Joliet }\end{array}$ & SW & $8.7 \mathrm{mi}$ & TSP & 34238 \\
\hline $\begin{array}{l}\text { Johns Manville } \\
\text { Prod Corp. }\end{array}$ & $\begin{array}{l}\text { Rt, } 6 \\
\text { Joliet }\end{array}$ & SW & $3.5 \mathrm{mi}$. & TSP & 73266.4 \\
\hline Economic Labs, Inc. & $\begin{array}{l}3001 \text { Channahon Rd. } \\
\text { Joliet }\end{array}$ & SW & $3.6 \mathrm{mi}$ & TSP & 5380.7 \\
\hline Persico Paving Co. & Moen Ave., Joliet & SW & $1.9 \mathrm{mi}$ & TSP & 1575.1 \\
\hline Com Ed-Joliet Sta. & $\begin{array}{l}\text { Patterson Rd. } \\
\text { Joliet Twp. }\end{array}$ & & & $\begin{array}{l}\mathrm{TSP} \\
\mathrm{SO}_{2} \\
\mathrm{NO}_{\mathrm{X}} \\
\mathrm{CO} \\
\mathrm{HC}\end{array}$ & $\begin{array}{r}209,558 \\
71,719 \\
68,883 \\
3,039 \\
909\end{array}$ \\
\hline $\begin{array}{l}\text { Olin Corp. } \\
\text {-Blackson Works }\end{array}$ & Patterson \& Laraway & & & TSP & 98,472 \\
\hline
\end{tabular}


2. Mobile Sources that may Influence the Site:

$\begin{array}{lcc}\text { Names of Roadways: } & \begin{array}{c}\text { Midland } \\ \text { Local Street }\end{array} & \begin{array}{c}\text { Campbell } \\ \text { Local Street }\end{array} \\ \begin{array}{l}\text { Distance of Roadway } \\ \text { from Site (ft) }\end{array} & \sim 250 & 115 \\ \text { Composition of Roadway } & \text { Asphalt } & \text { Asphalt } \\ \text { Number of Traffic Lanes } & 2 & 2 \\ \text { Average Daily Traffic } & 2000 & <2000 \\ \text { Average Vehicle Speed (mph) } & 30 & 25 \\ \text { One or Two Way Traffic } & \text { Two } & \text { Two } \\ \text { Number of Parking Lanes } & \text { None } & \text { None } \\ \text { Are Parking Lanes Used } & & \text { No } \\ \text { For Traffic Part of Day? } & \text { No } & \text { No } \\ \text { Is Dust Visibiity Retained } & \text { No } & \text { No } \\ \text { Does Roadway Have Curb } & \text { No } & \end{array}$

3. Area Sources that may Influence the Site:

\begin{tabular}{lcccc}
\multicolumn{1}{c}{ Type of Source } & $\begin{array}{c}\text { Direction } \\
\text { from } \\
\text { Site }\end{array}$ & $\begin{array}{c}\text { Distance } \\
\text { from } \\
\text { Site }\end{array}$ & Pollutant \\
\cline { 2 - 3 } $\begin{array}{l}\text { Chicago Metropolitan } \\
\text { Area }\end{array}$ & $\mathrm{NE}$ & & $30 \mathrm{mi}$ & $\mathrm{TSP}, \mathrm{SO}_{2}, \mathrm{Nn}, \mathrm{CO}, \mathrm{O}_{3}$ \\
Cornfield & $\mathrm{S}$ & $\mathrm{TSP}$
\end{tabular}


D. Topography/Obstructions

1. General Characteristics Over a 2 Mile Radius From the Site: Smooth

2. Topographic Features that Influence the Site: (Types - hills, valleys, depressions, bodies of water, ridges, cliffs)

Type

\begin{tabular}{ll} 
Size & Direction \\
\hline
\end{tabular}

Distance From Site

None

3. Obstructions to Wind Flow

(Types - buildings, trees, ridges, cliffs)

Type

\section{$\underline{\text { Size }}$}

Direction

Distance

From Site.

From Site

None

4. Comments

The 2 story section of roof over the gymnasium could prove to be an influence to wind flow.

E. Distance to the nearest National Weather Service (NWS) Site.

Here are the distances and directions to the nearest NWS observing site from the inhalable particulate monitor in Joliet. Distances are in statute miles; directions are degrees from true north.

Site Address

Pershing School

Midland and Campbe11
Nearest NWS Site

O'Hare Airport/Chicago (ORD)
Directions \& Distances to NWS

Midway Airport (MDW) is about 25 miles northeast of Joliet (to MDW from Joliet, $050^{\circ}$ at $25.5 \mathrm{mi}$ ).

Dupage Airport (DPA) is about 28 miles north-northwest of Joliet (to DPA from Joliet, $340^{\circ}$ at $28 \mathrm{mi}$ ).

Data from both sites are available to the IEPA through Service "A", but neither is a NWS site (though MDW used to be), and no LCDs are issued for them. 


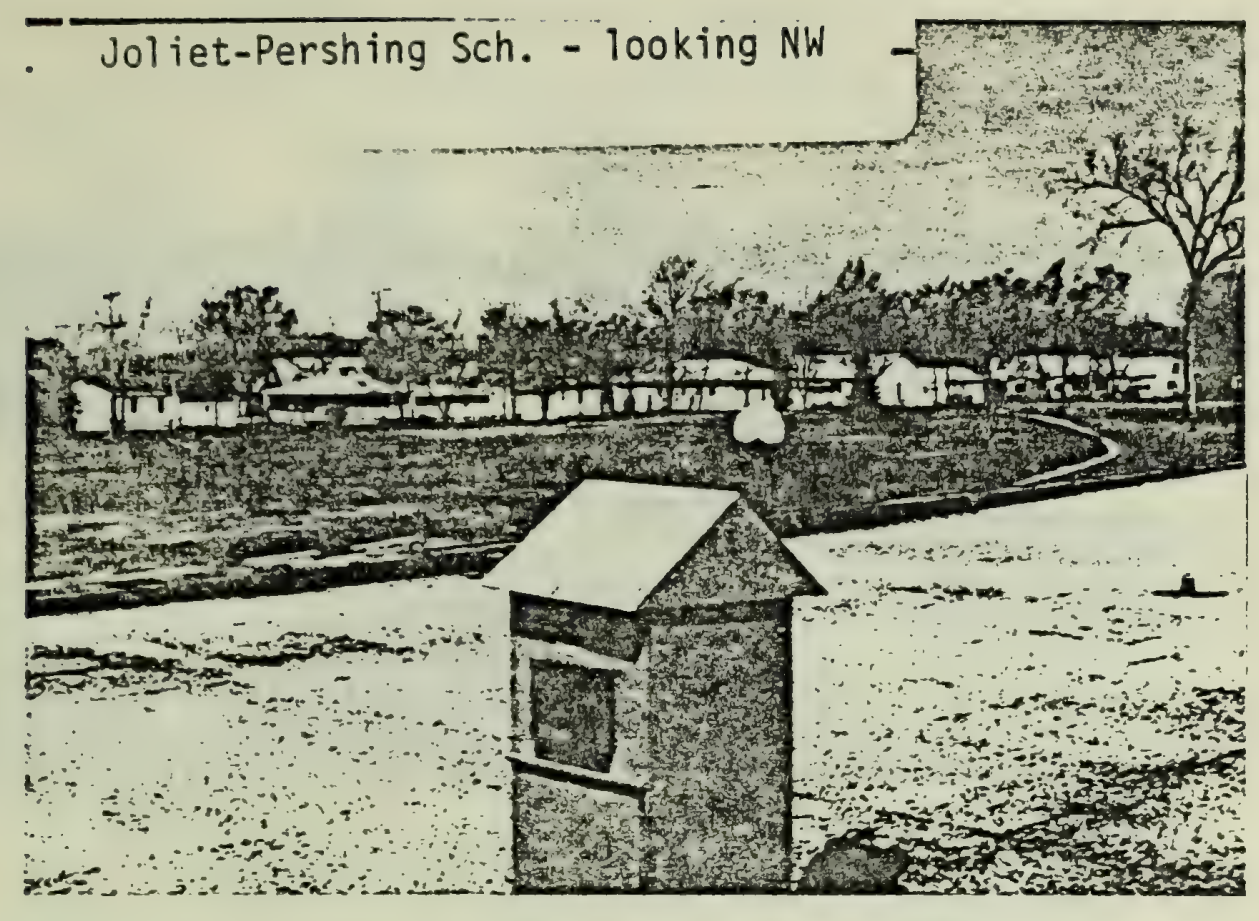

Joliet - North West

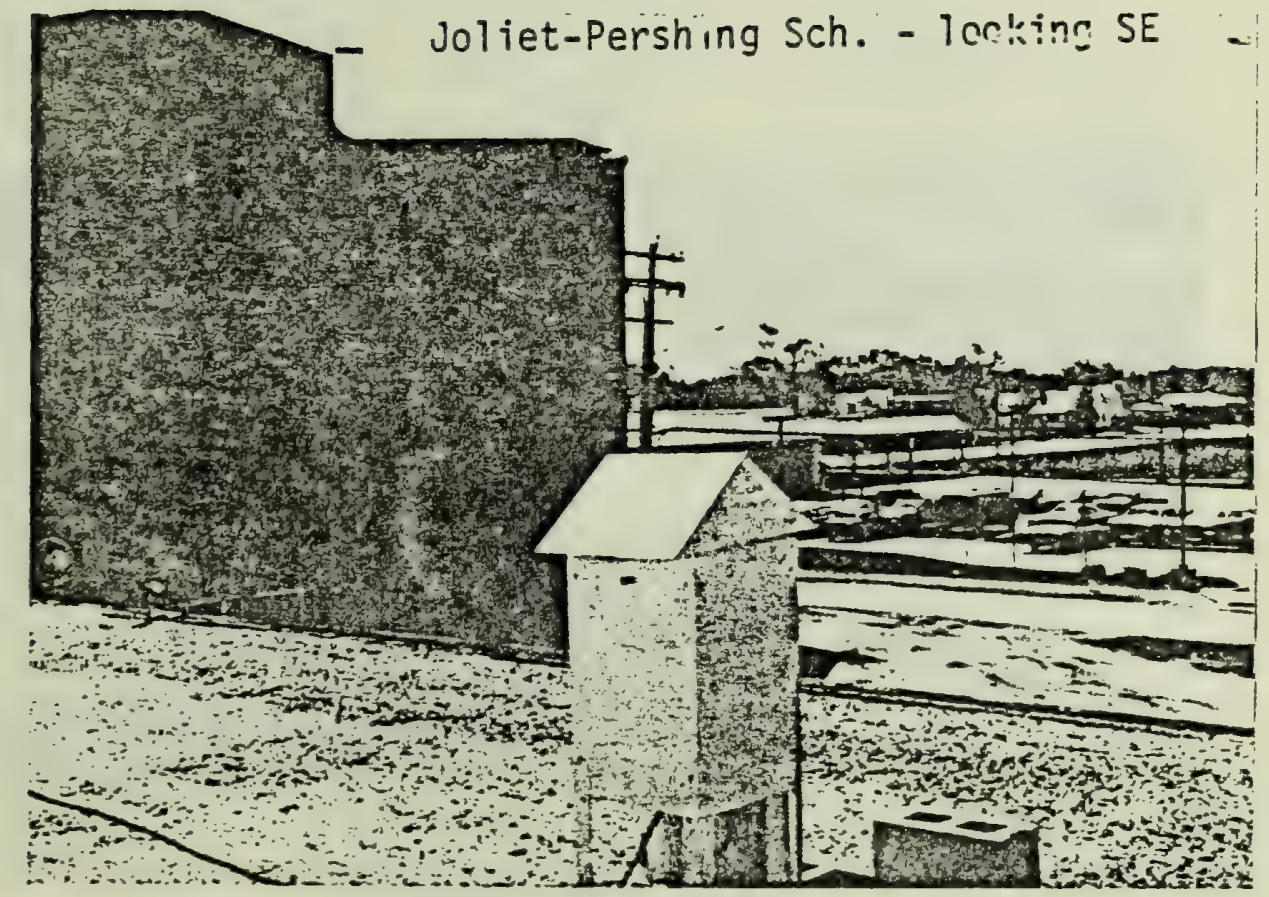




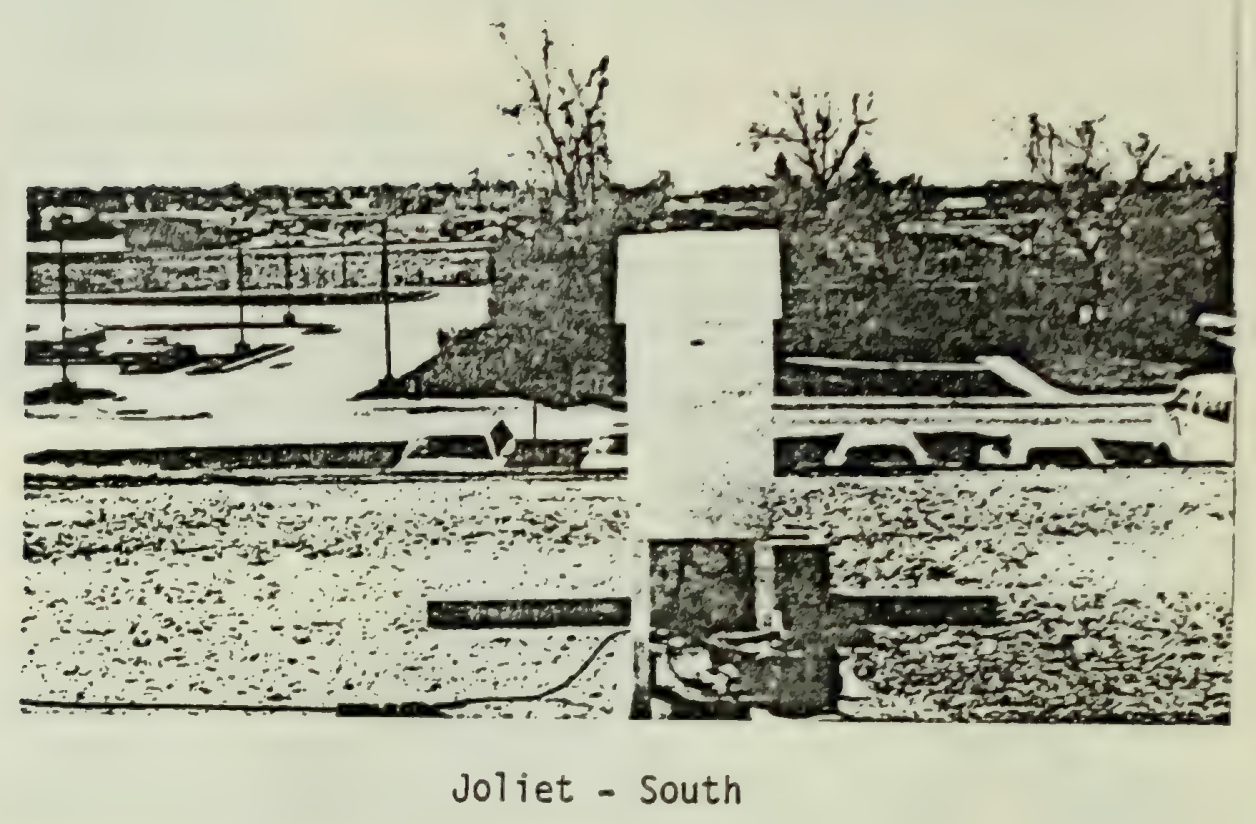

- Joliet-Pershing Sch. - looking SW -

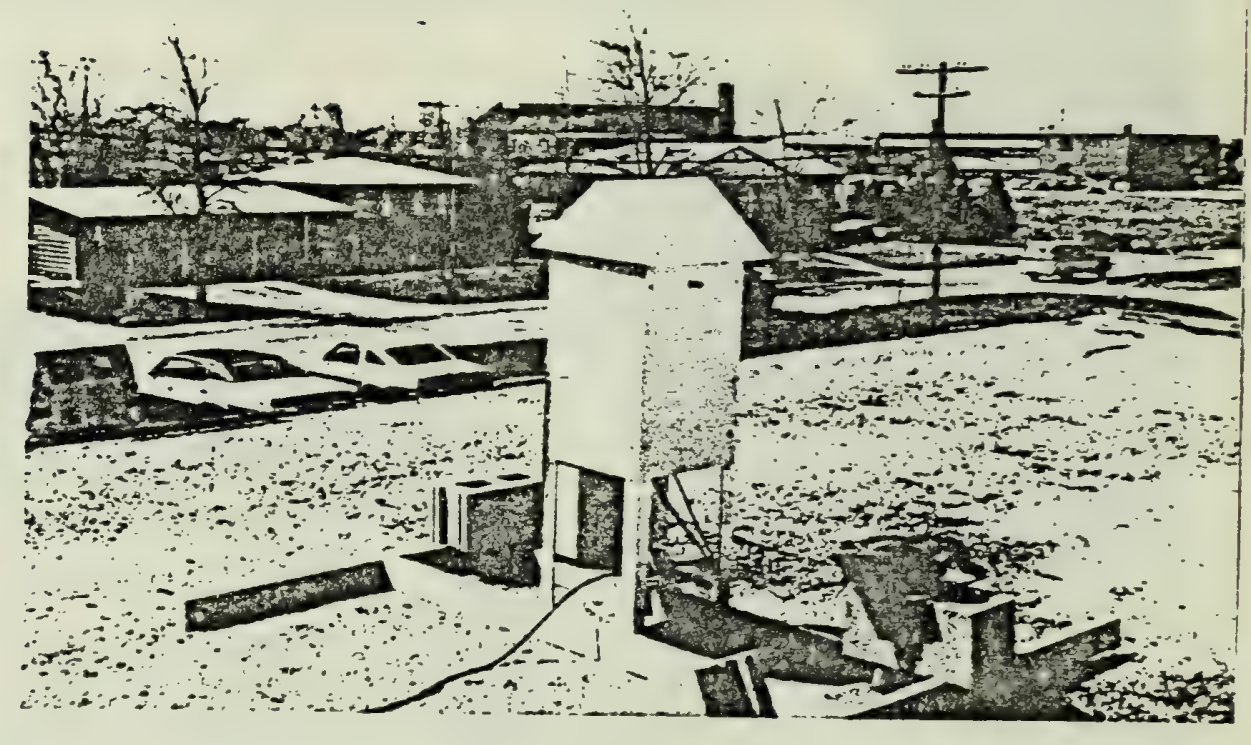

Joliet - South West 
$\begin{array}{cclc}\text { Particulate: Minerals } & 96.7 & \text { Combustion } & 22.3 \\ \text { Biological } 5 & \text { Other } & - \\ \text { Low temperature ashing, \% loss } & \end{array}$
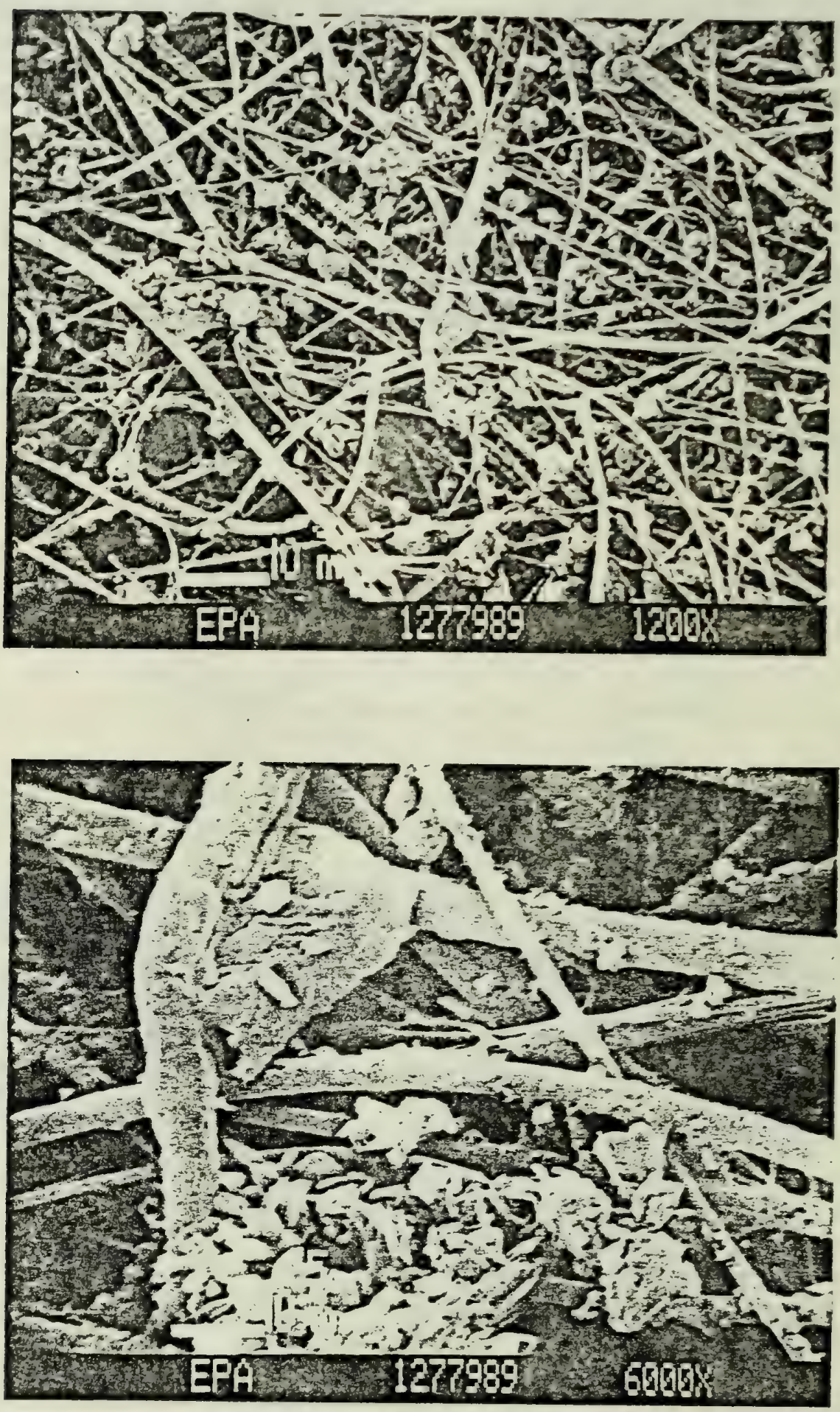
Inhalable Particulate

$$
\mu g / m^{3}
$$
Particulate: Minerals
12.4
Combustion
17.5
Biological 43.1
Other

Low temperature ashing, \% loss

31.4
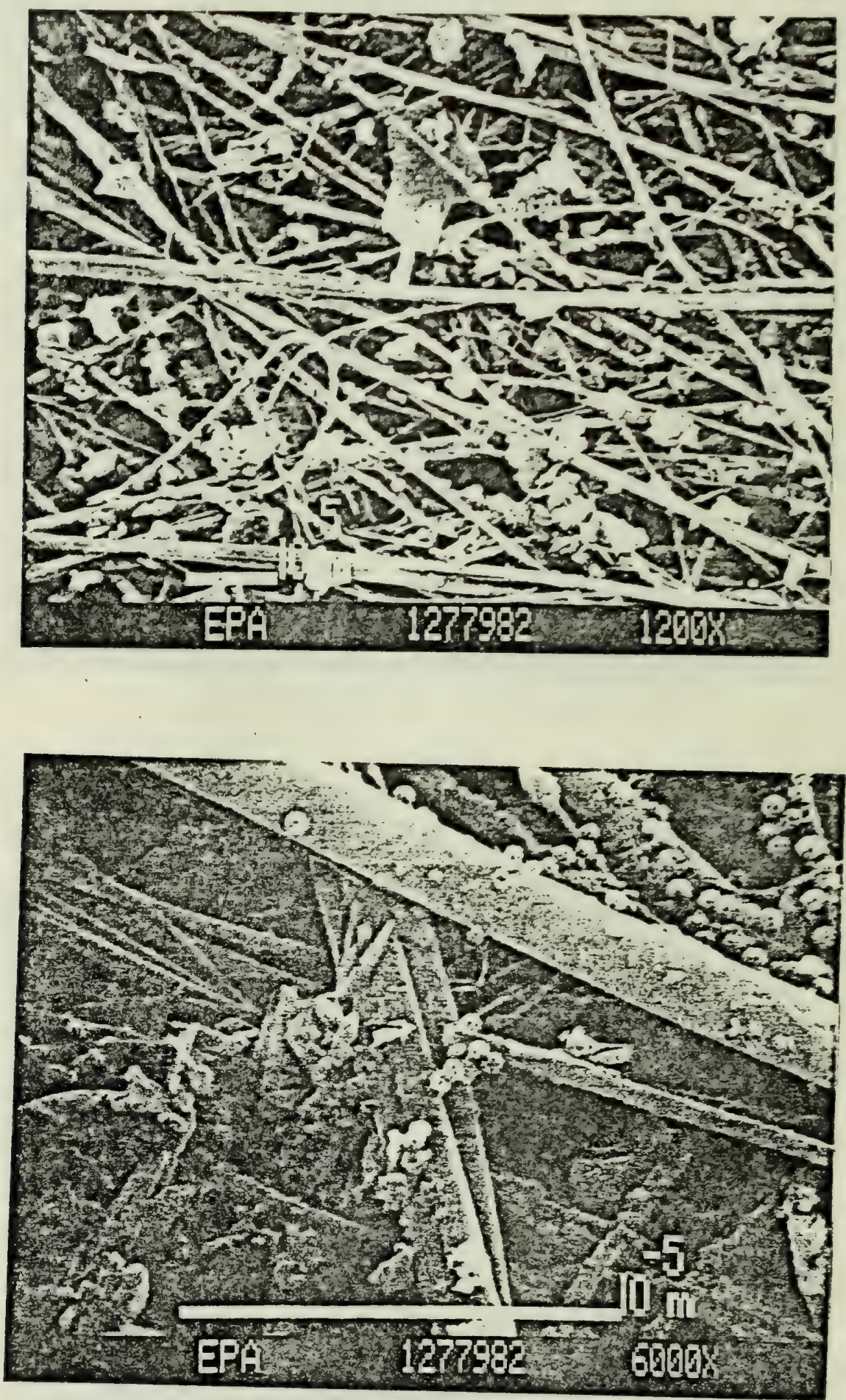
A. Site Identification

APPENDIX C

1. City: Rockford

2. Site Name and Address: Charles Beyer School, 333 15th Ave.

3. County: Winnebago Township: Rockford

4. USGS Topographical Map Name and Scale, Rockford North,1:24000

5. Site Elevation (Feet) : 735

B. Site Classification/Representativeness

1. Dominating Influence on Site: Industrial, Mobile

C. 1. Stationary Sources that may Influence Site:

\begin{tabular}{|c|c|c|c|c|c|}
\hline Name of Source & Location & $\begin{array}{l}\text { rection } \\
\text { From } \\
\text { Site }\end{array}$ & $\begin{array}{c}\text { Distance } \\
\text { From } \\
\text { Site } \\
\end{array}$ & Pollutant & $\begin{array}{l}\text { Emissions } \\
\left(\text { Tons } / Y_{r}\right) \\
\end{array}$ \\
\hline Amerock Corp. & $\begin{array}{l}4000 \text { Auburn } \\
\text { Rockford }\end{array}$ & NNW & $3.0 \mathrm{mi}$ & $\begin{array}{l}\text { TSP } \\
\mathrm{NO}_{\mathrm{X}} \\
\mathrm{HC}\end{array}$ & $\begin{array}{r}108 \\
93 \\
868\end{array}$ \\
\hline Barber-Colman Co. & $\begin{array}{l}1300 \text { Rock St. } \\
\text { Rockford }\end{array}$ & N & $1.2 \mathrm{mi}$ & $\begin{array}{l}\text { TSP } \\
\mathrm{NOX} \\
\mathrm{HC}\end{array}$ & $\begin{array}{r}299 \\
47 \\
101\end{array}$ \\
\hline Elco Industries & $\begin{array}{l}111 \text { Samuel son } \\
\text { Rd., Rockford }\end{array}$ & SSE & $3.0 \mathrm{mi}$ & $\begin{array}{l}\text { TSP } \\
\text { HC }\end{array}$ & $\begin{array}{r}152 \\
2,184\end{array}$ \\
\hline Estwing Mfg. & $\begin{array}{l}2647 \text { 8th St. } \\
\text { Rockford }\end{array}$ & SSE & $1.2 \mathrm{mi}$ & TSP & 101 \\
\hline Forgings \& Stampings. & $\begin{array}{l}1025 \text { 23rd Ave } \\
\text { Rockford }\end{array}$ & - SE & $0.9 \mathrm{mi}$ & TSP & 112 \\
\hline Greenlee Bros. Co. & $\begin{array}{l}2136 \text { 12th St. } \\
\text { Rockford }\end{array}$ & ESE & $1.4 \mathrm{mi}$ & $\begin{array}{l}\text { TSP } \\
\text { CO }\end{array}$ & $\begin{array}{r}1,671 \\
257\end{array}$ \\
\hline Keisey-Hayes Co. & $\begin{array}{l}302 \text { Peoples } \\
\text { Ave. } \\
\text { Rockford }\end{array}$ & $S$ & $0.6 \mathrm{mi}$ & $\begin{array}{l}\text { TSP } \\
\text { CO }\end{array}$ & $\begin{array}{l}46,510 \\
14,805\end{array}$ \\
\hline Olson Pattern \& Foundry & $\begin{array}{r}1617-1711 \\
\text { Magnolia } \\
\text { Rockford }\end{array}$ & w & $0.2 \mathrm{mi}$ & TSP & 184 \\
\hline
\end{tabular}


Accurate Die Casting

$$
\begin{aligned}
& 2025 \\
& \text { Kishwaukee } \\
& \text { Rockford }
\end{aligned}
$$

SE

$0.4 \mathrm{mi}$.

TSP

Rockford Can Co. Quaker Rd. SSW Rockford

$0.7 \mathrm{mi}$.

TSP

$\mathrm{NO}_{X}$

8,844

Rockford Prod. Corp. 707 Harrison SSE

$0.9 \mathrm{mi}: \quad$ TSP

223

Ave.

Rockford

HC

Sal1 Bros. Co.

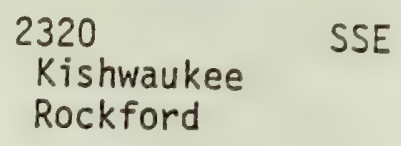

$0.7 \mathrm{mi} . \quad$ TSP

128

Weyerhauser

2100 23rd ESE
Ave.
Rockford

$1.7 \mathrm{mi} . \quad$ TSP $\quad 1,173$

Joseph Behr \& Sons

110 Seminary N

St. Rockford

$0.5 \mathrm{mi} . \quad$ TSP

378

Mattison Mach. Wks.
2235 SSE
Kishwaukee
Rockford

$\begin{array}{ll}0.8 \mathrm{mi} . & \text { TSP } \\ & \text { CO }\end{array}$

146

365

Longuiew Fibre co.

1878 Elmwood NNE Rockford

$5.7 \mathrm{mi} . \quad$ TSP

4,270

Rockford Blacktop
4102 S. Main SSW Rockford

$3.1 \mathrm{mi.}$ TSP

895

Kent Feeds

1612 S. Bend $S$

Rd. Rockford

$5.0 \mathrm{mi} . \quad$ TSP

21,406

Rein, Schultz \& Dahl
801 Beale Ct. SSW Rockford

$4.5 \mathrm{mi} . \quad$ TSP

2,765 
2. Mobile Sources that may Influence the Site:

\begin{tabular}{|c|c|c|}
\hline Names of Roadways: & 16th Ave. & Seminary \\
\hline Arterial Street & Local Street & Local Street \\
\hline $\begin{array}{l}\text { Distance of Roadway } \\
\text { from Site (ft) }\end{array}$ & 180 & 600 \\
\hline Composition of Roadway Asphalt & Asphalt & Asphalt \\
\hline Number of Traffic Lanes 4 & 2 & 2 \\
\hline Average Daily Traffic 8000 & $<1000$ & 3000 \\
\hline Average Vehicle Speed (mph) 30 & 15 & 30 \\
\hline One or Two Way Traffic Two & Two & Two \\
\hline Number of Parking Lanes None & Two & Two \\
\hline $\begin{array}{l}\text { Are Parking Lanes Used } \\
\text { For Traffic Part of Day? N/A }\end{array}$ & No & West Side \\
\hline Is Dust Visibiity Retained No & No & No \\
\hline Does Roadway Have Curb Yes & Yes & Yes \\
\hline
\end{tabular}

3. Area Sources that may Influence the Site:

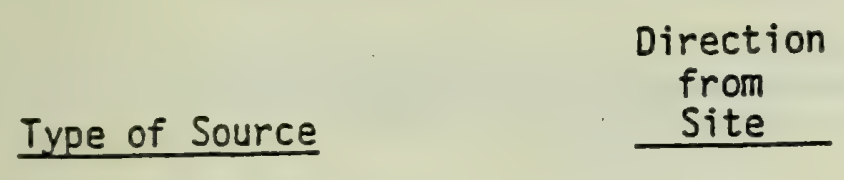

Parking Lot, Paved

Parking Lot, Paved

Parking Lot, Unpaved

Parking Surrounding Building

Truck Yard

Misc. Parking, Paved and

Unpaved Around Factories
Distance

from

Site

Pollutant

TSP

TSP

TSP

TSP

TSP

TSP 
D. Topography/Obstructions

1. General Characteristics Over a 2 Mile Radius From the Site: Smooth

2. Topographic Features that Influence the Site: (Types - hills, valleys, depressions, bodies of water, ridges, cliffs)

$\begin{array}{lccc}\text { Type } & \text { Size } & \begin{array}{c}\text { Direction } \\ \text { From Site }\end{array} & \begin{array}{c}\text { Distance } \\ \text { From Site }\end{array} \\ \text { Rock River } & 400-700^{\prime} & \text { East } & 2000-2500^{\prime}\end{array}$

3. Obstructions to Wind Flow

(Types - buildings, trees, ridges, cliffs)

Type

\begin{tabular}{lll} 
Size & Direction & Distance \\
& From Site & From Site \\
\hline
\end{tabular}

None

E. Distance to the nearest National Weather Service (NWS) Site.

Here are the distances and directions to the nearest NWS observing site from the inhalable particulate monitor in Rockford. Distances are in statute miles; directions are degrees from true north.

Site Address

Beyer Elem. School

333 15th Ave.
Nearest NWS Site

Rockford Airport (RFD) $\quad 185^{\circ}$ at $3.4 \mathrm{mi} .(\mathrm{s}$ )
Directions and Distance to NWS 


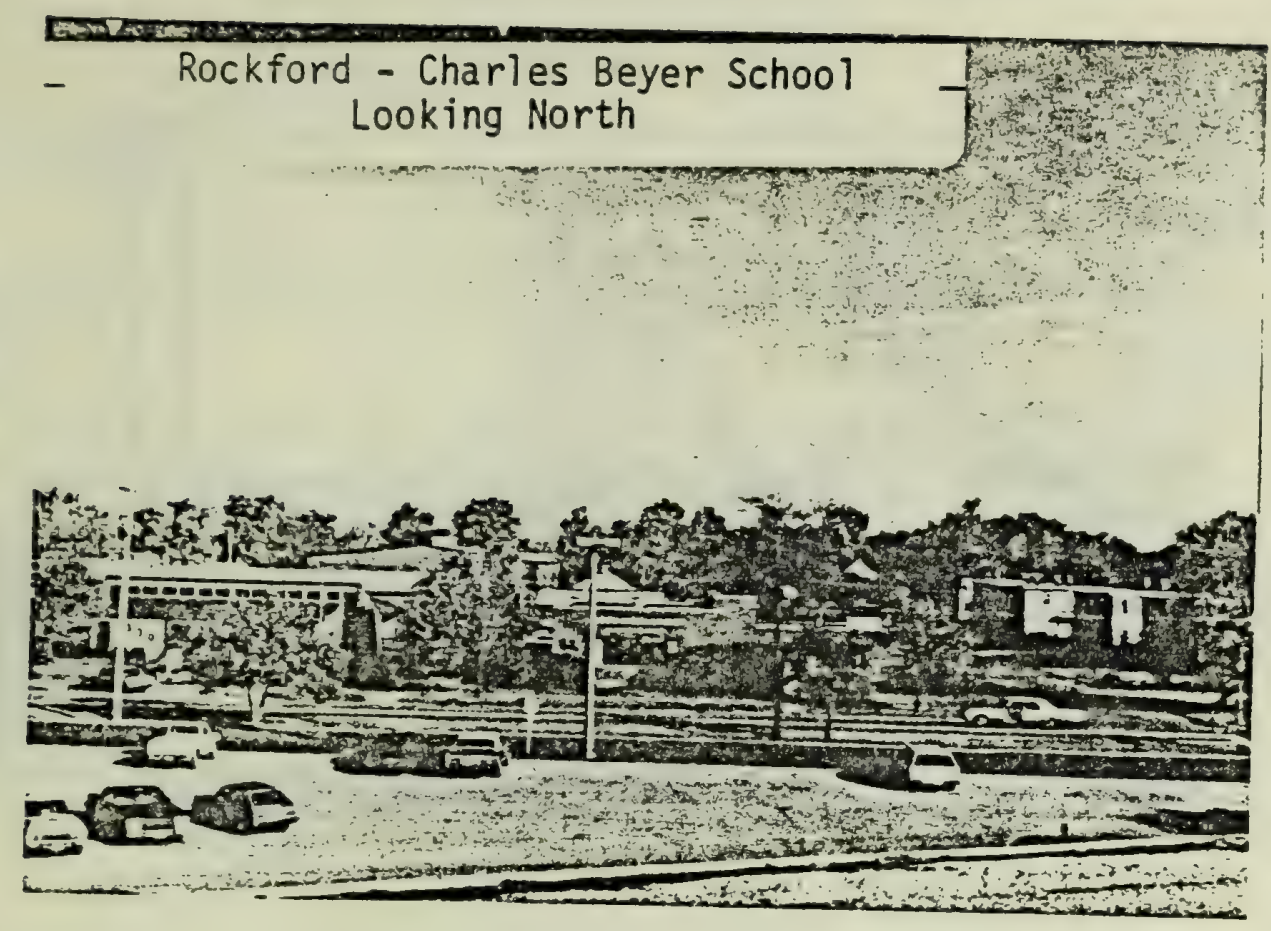

Rockford - North

Rockford - Charles Beyer School
Looking SW

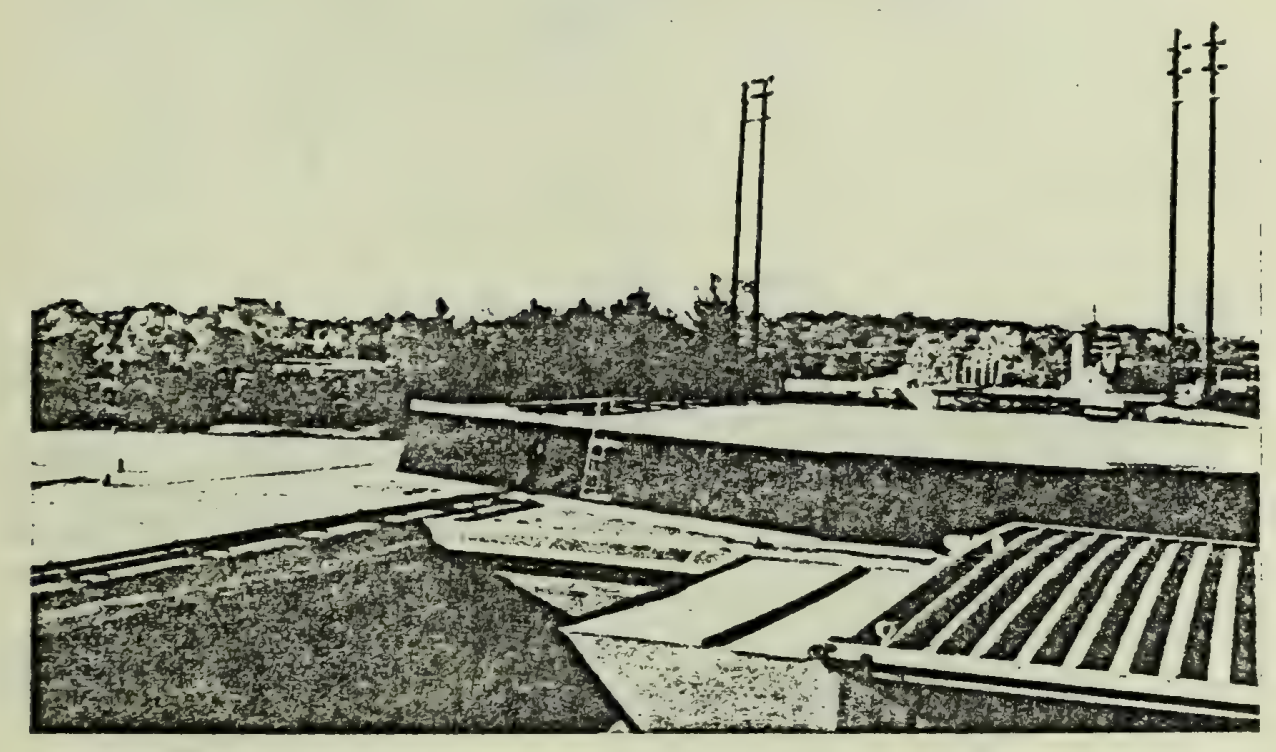

Rockford - South West 


\section{Rockford - Charles Beyer School \\ Looking West}

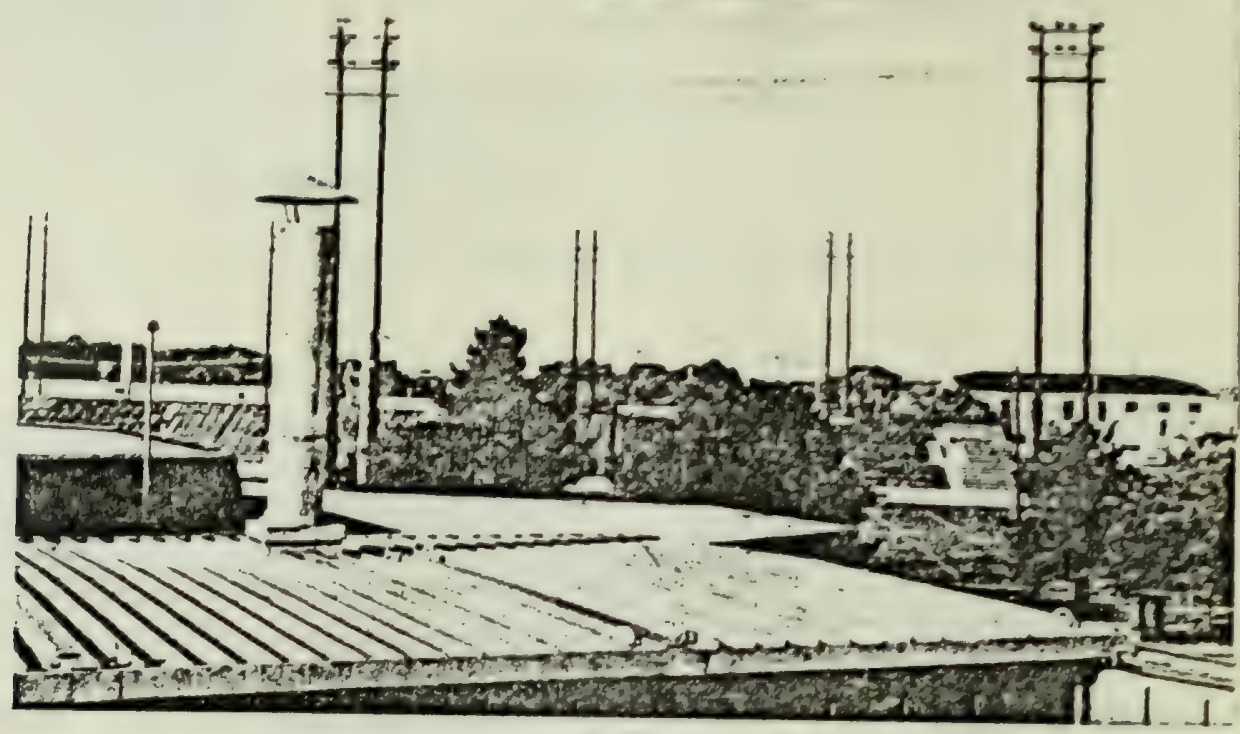

Rockford - West

\section{- Rockford - Charles Beyer School \\ Looking NW}

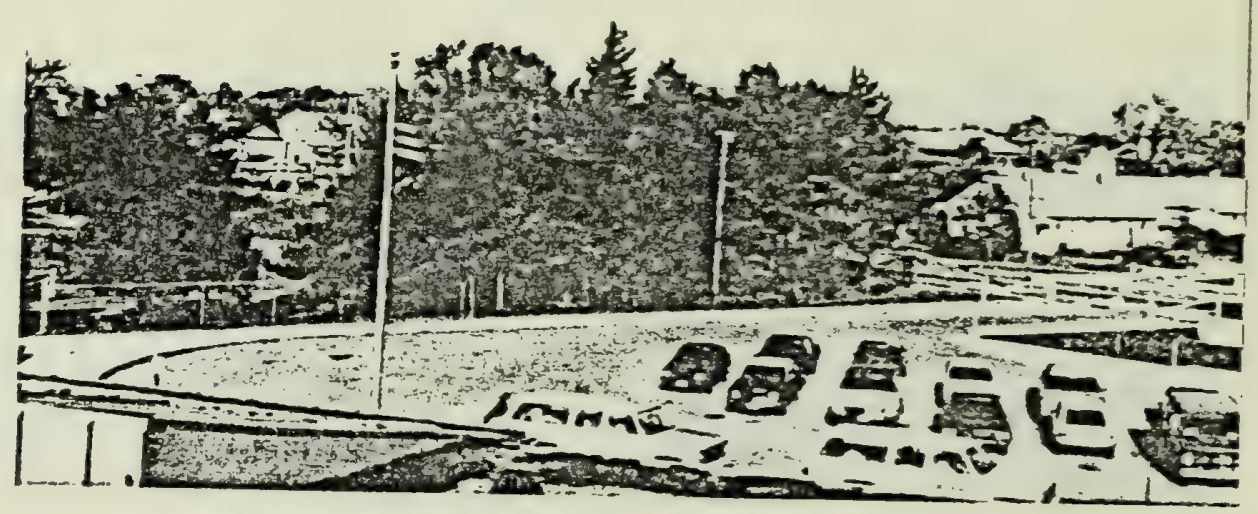

Rockford - North West 


\begin{tabular}{|c|c|c|c|c|}
\hline $\begin{array}{l}\text { III_TER NLIMEER : } 122999 \% \\
\text { 1.TLLELT ION DATE : } 05 / 22.81 \\
:==========================\end{array}$ & $\begin{array}{l}\text { NETWLIK : } \\
\text { SITE: } \\
\text { OE.JEITIVE } \\
============\end{array}$ & $\begin{array}{l}\text { FIOUKFORI, } \\
\text { AB: : } 25 \\
=========\end{array}$ & $\begin{array}{l}\text { EYERSEHIOL } \\
:=========\end{array}$ & \\
\hline COMFIINENT & $\begin{array}{l}\text { AVERAGE } \\
\text { FAFT. SIZE }\end{array}$ & RANLE & $\begin{array}{l}\text { WEITIHT } \\
\text { PEFILENT }\end{array}$ & $\begin{array}{l}\text { Number } \\
\text { Percent }\end{array}$ \\
\hline \multicolumn{5}{|l|}{ MINEFAL 5} \\
\hline QLIARTZIFEI_MSFARS & 17. 8 & 2. $5-57.6$ & 49 & 26 \\
\hline LIMESTINE & 9. 8 & 1. $8-28.6$ & 11 & 46 \\
\hline IRON OXIDES & 10. 1 & 2. $5-28.8$ & 2 & 2 \\
\hline DTHER MINERALS & & & 0 & 0 \\
\hline \multicolumn{5}{|l|}{ COMEUSTION PRODUIT:TS } \\
\hline SDOT & 19.0 & 2. $5-57.6$ & 11 & 19 \\
\hline GLASSY FLYASH SFHEFES & & & 0 & 0 \\
\hline \multicolumn{5}{|l|}{ BIULDI.STCAL } \\
\hline PILLEN \& SFIIFE: & $1 \% .6$ & 7. $z-40.7$ & 2 & 1 \\
\hline PLANT TISEIIE & 3.1 & 10. $2-81.5$ & 26 & 6 \\
\hline STAFILH & 12. 3 & 10. $2-14.4$ & $<1$ & 1 \\
\hline \multicolumn{5}{|l|}{ MISICELLANEDIS } \\
\hline FEFIRDUIS METAL. & & & 0 & 0 \\
\hline FUUEEER & & & 0 & 0 \\
\hline
\end{tabular}

FARTIILE SIZE DIETRIEUITIIN

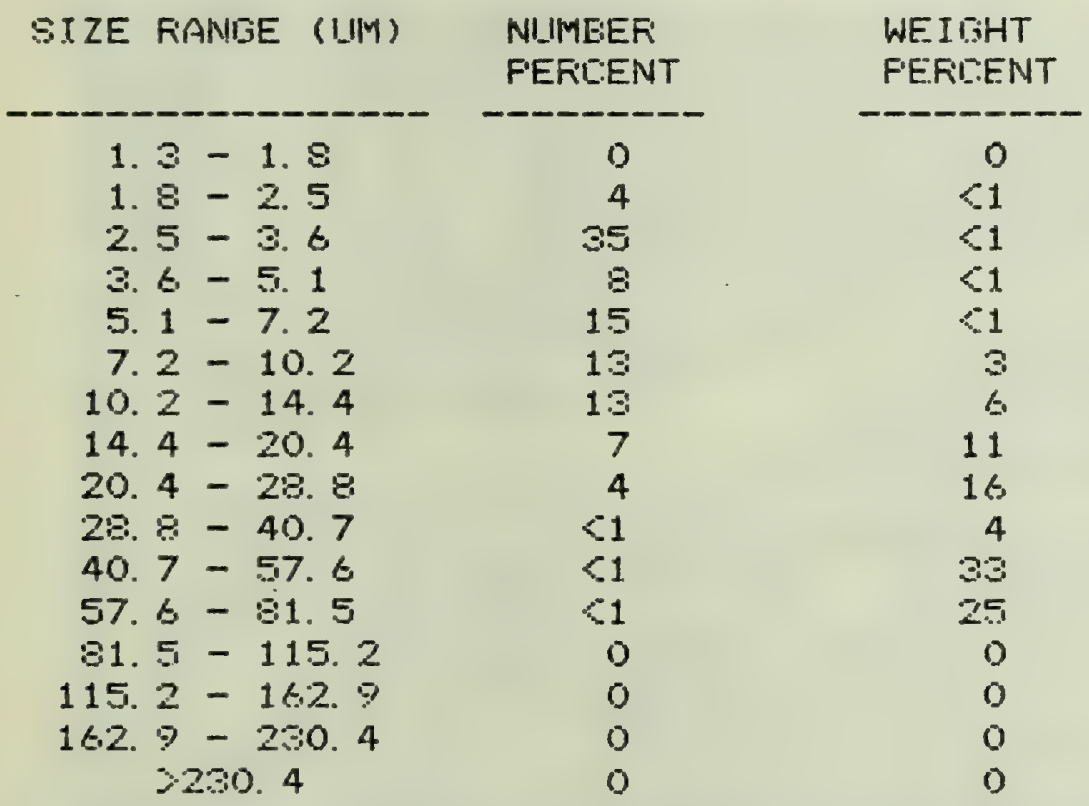


Total Suspended Particulate $\mu \mathrm{g} / \mathrm{m}^{3}$

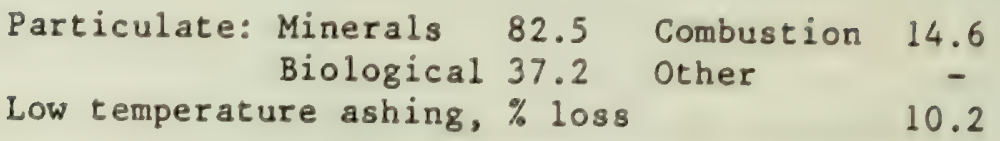
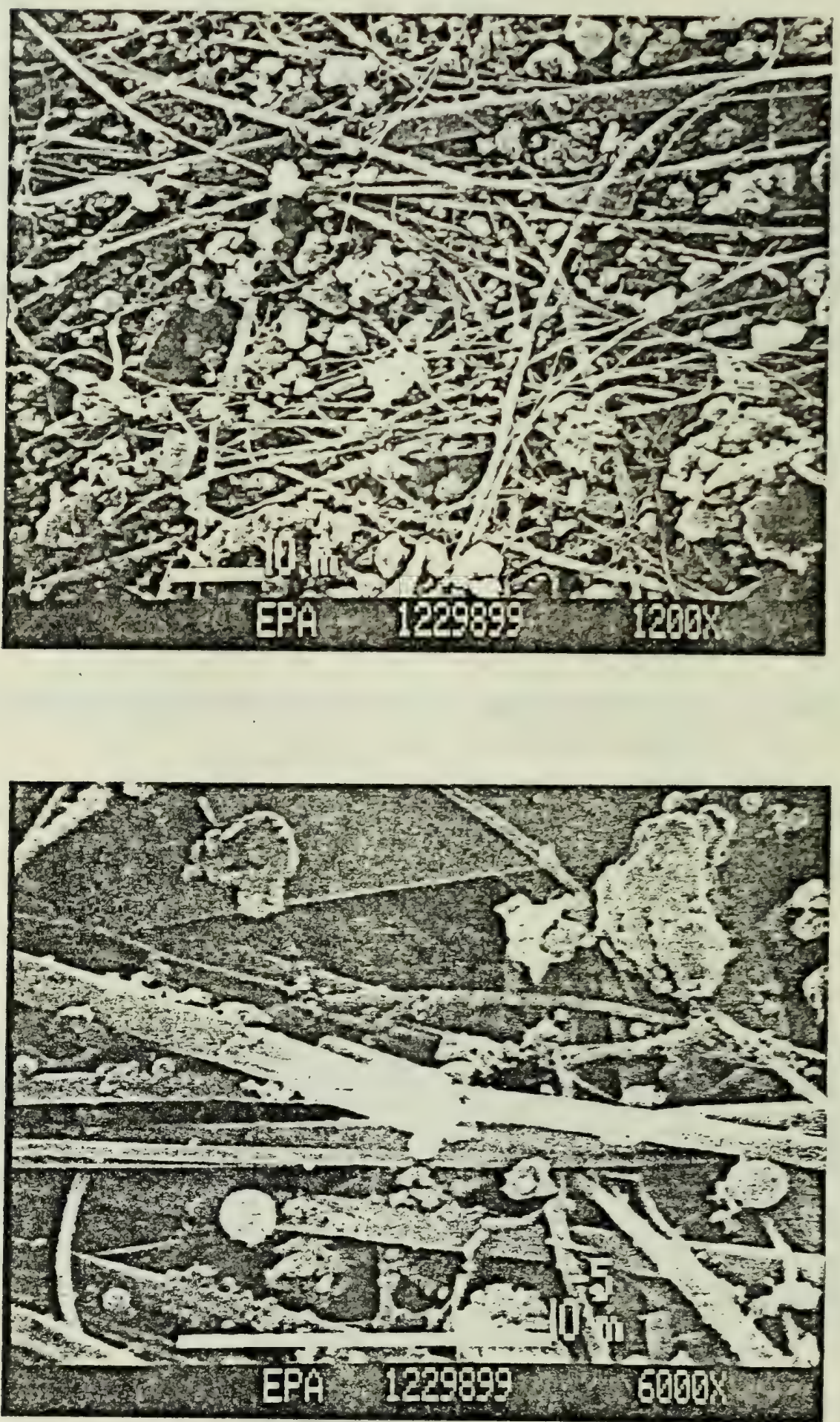
Inhalable Particulate

$\mathrm{ug} / \mathrm{m}^{3}$

124

\begin{tabular}{|c|c|c|c|}
\hline 1culate: & $\begin{array}{l}\text { Minerals } \\
\text { Biological }\end{array}$ & $\begin{array}{r}99.2 \\
9.9\end{array}$ & $\begin{array}{l}\text { Combustion } \\
\text { Other }\end{array}$ \\
\hline
\end{tabular}
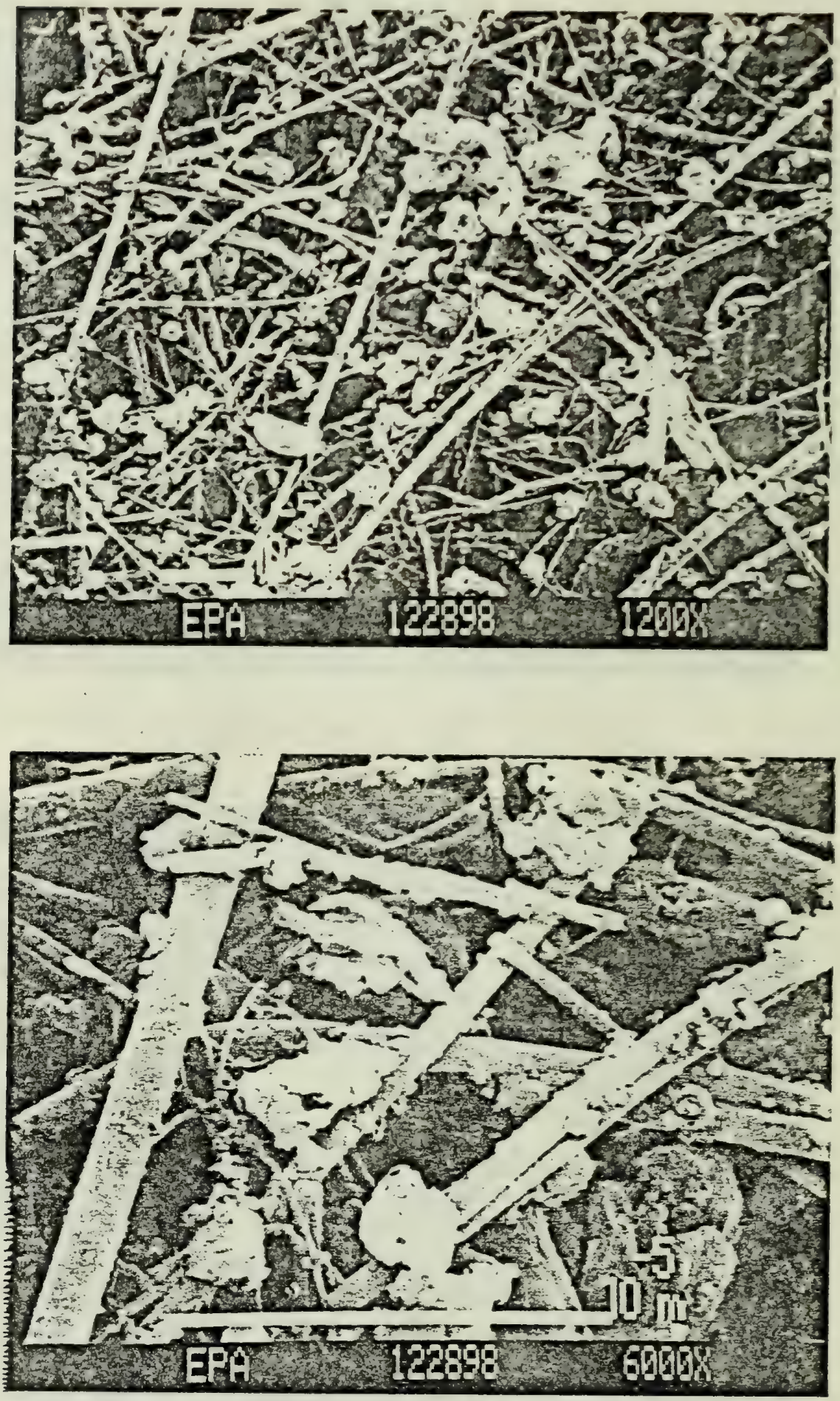


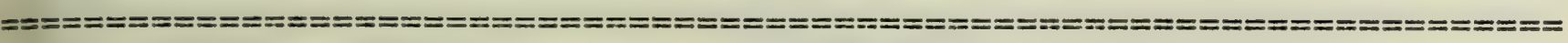

ILTER NUMEER : 1282741

OLLECTION DATE : $12 / 18 / 81$

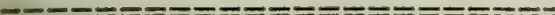

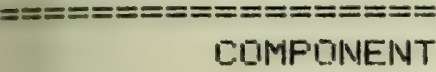

NETWOFK :

SITE : DE.IELTIVE MAT

FIOKFDRD/EEYEFEI:HCIRL.
AVERALIE

FART. SIZE
RANGE

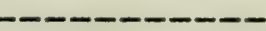

\section{5 \\ 12. 6 \\ 12. 3}

OTHER MINEFALS

RUIAFTZ/FFI..DSPAFS

LIMESTONE.

IRON DXIJIES
DTHER MINERALS

COMBUSTION FFONULTS

SOOT

GLASSY FLYASH SFHEFES
12. 3

7. 4

15. 8

12. 3
3. $6-40.7$

1. $8-40.7$

3. $6-28.8$

3. $6-29.9$

7

0

10

0

\section{BIOLONICAL.}

PULLEN \& SFURE:

FLANT TISSUIE

STARC.H
5. $1-10.2$

7. $2-28.8$

10. $2-14.4$

$\begin{array}{rr}<1 & 1 \\ 4 & 2 \\ <1 & <1\end{array}$

WEIGHT

FERCENT
Number

Percent

\section{MISCELLANENIS:}

FERRIDUS METAL

RUEEEER

FAFTILLE SIZE DISTRIEUUTION

\begin{tabular}{|c|c|c|c|}
\hline SIIZE & FIAIVISE (IIM) & $\begin{array}{l}\text { NLIMEER } \\
\text { F'EFICENT }\end{array}$ & $\begin{array}{l}\text { WEIOHT } \\
\text { FERIOENT }\end{array}$ \\
\hline & $3-1.8$ & 0 & 0 \\
\hline & $8-2.5$ & 29 & $<1$ \\
\hline 2. & $5-3 b$ & 16 & $<1$ \\
\hline 3. & $6-5.1$ & 13 & $<1$ \\
\hline 5. & $1-7.2$ & 11 & 2 \\
\hline 7. & $2-10.2$ & 12 & 6 \\
\hline 10. & $2-14.4$ & $\varepsilon$ & 11 \\
\hline 14. & $4-20.4$ & $\Xi$ & 31 \\
\hline 20. & $4-28$. & 3 & 33 \\
\hline 28 & $8-40.7$ & $<1$ & 16 \\
\hline 40. & $7-57.6$ & 0 & 0 \\
\hline 57. & $b-81.5$ & 0 & 0 \\
\hline 81. & $5-115.2$ & 0 & 0 \\
\hline 115. & $2-16.2 .9$ & 0 & 0 \\
\hline $1 \leqslant 2$ & $9-230.4$ & 0 & 0 \\
\hline & $>230.4$ & 0 & 0 \\
\hline
\end{tabular}


Total Suspended Particulate $\mu g / \mathrm{m}^{3}$

$\begin{array}{cclc}\text { Particulate: Minerals } & 25.5 & \text { Combustion } & 2.0 \\ \text { Biological } & 1.2 & \text { Other } & - \\ \text { Low temperature ashing, } \% \text { loss } & & 14.3\end{array}$
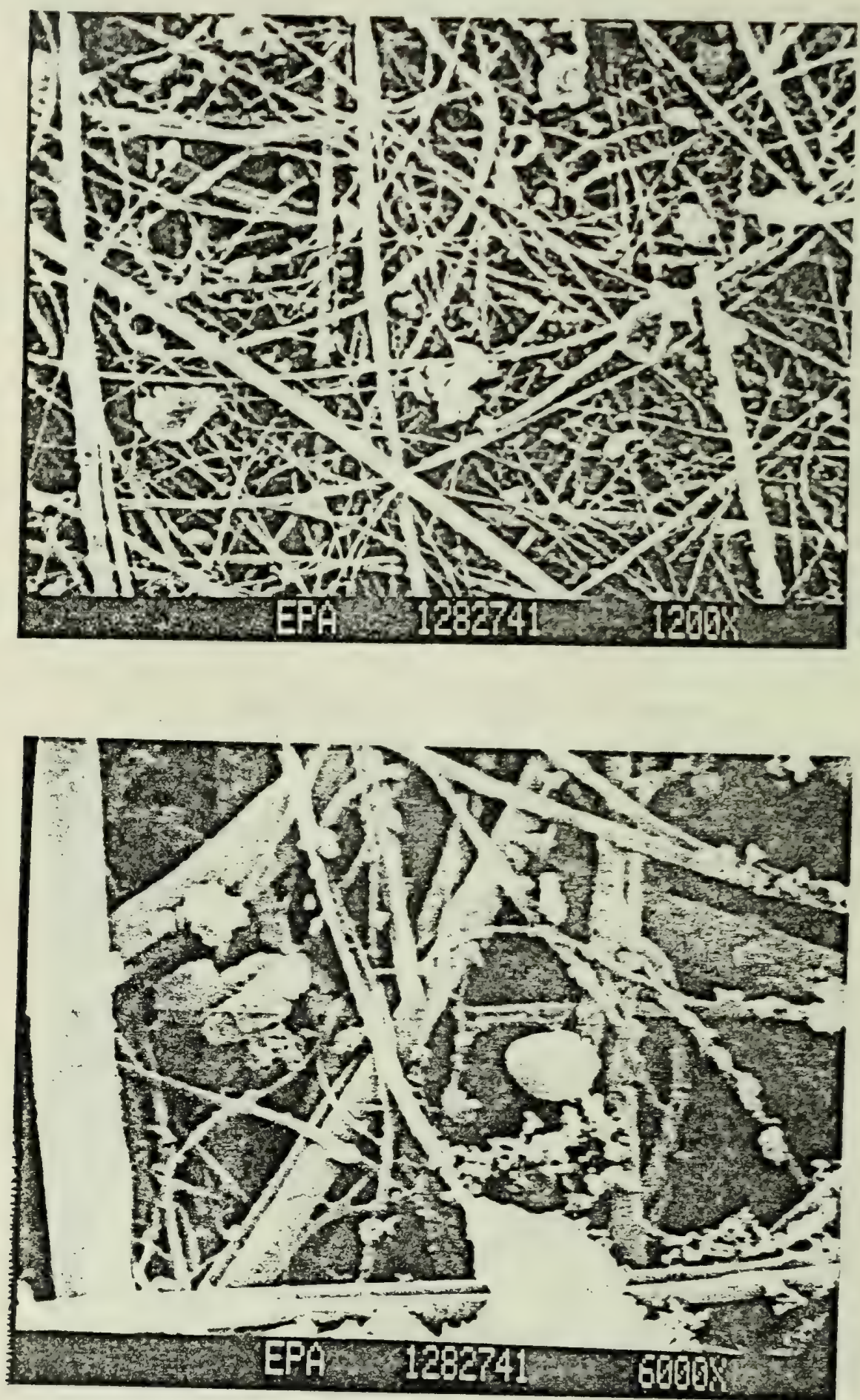


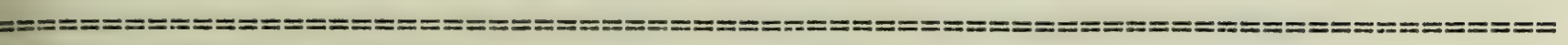

ILTER NUMEER : 1282740 .

NETWIRK :

SITE : RIOCKFIRD/EEYERELHIIIL

DE.IECTIVE MAB. : 25

OLLECTION RATE : $12 / 18 / 81$

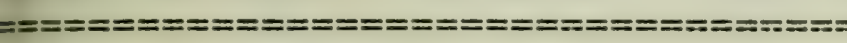
COMPONENT

AVERAGE

FAFT. SIZE.
FIAIVIEE

$==ニ=ニ====$
FiAlViE.

WEIGHT

FERIEENT
Number

Percent

\section{MINERALS}

QUIARTZ/FEI_DSFAFIS

LIMESTINE

3. $6-14.4$

IRON OXINES

OTHER MINEFIALS

6. 9

2. $5-14.4$

1. $3-5.1$

COMEUSTION FFIOLII:TS

SIDTT

GLASSY FLYASH SFHEFES

6. 9

2. 5-14. 4

14

0

11

$\Leftrightarrow 1$

$<1$

72

1

0

BIOL_OUICAI.

POLLEN \& SFIIRES

PLANT TISSIIE

STAFICH

6. 9

2. $5-14.4$

21. 7

7. $2-40.7$

5. $1-14.4$

12

9. 2

$<1$

MISLELLANEDII:-

FEFRTIUIS METAL.

RIIEBER

FAFTIILE SIZE LISTFIEUTIIIN

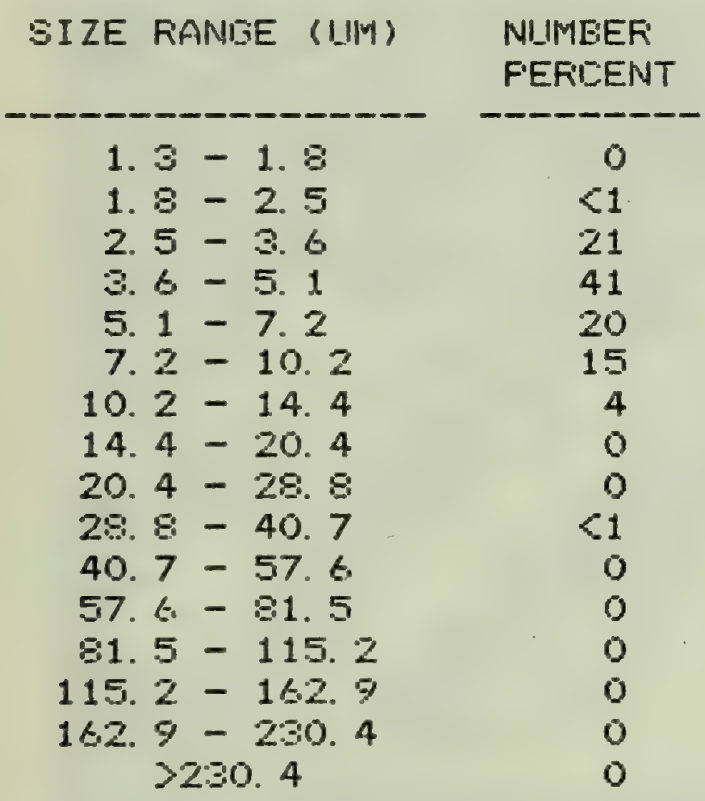

\begin{tabular}{c} 
WEIGHT \\
FE.RIENT \\
\hline 0 \\
01 \\
2 \\
14 \\
17 \\
34 \\
22 \\
0 \\
0 \\
10 \\
0 \\
0 \\
0 \\
0 \\
0 \\
0
\end{tabular}


Inhalable Particulate

$\begin{array}{cccc}\text { Particulate: Minerals } & 17.3 & \text { Combustion } & 3.4 \\ \text { Biological } & 3.1 & \text { Other } & - \\ \text { Low temperature ashing, } \% 108 s & & 28.4\end{array}$
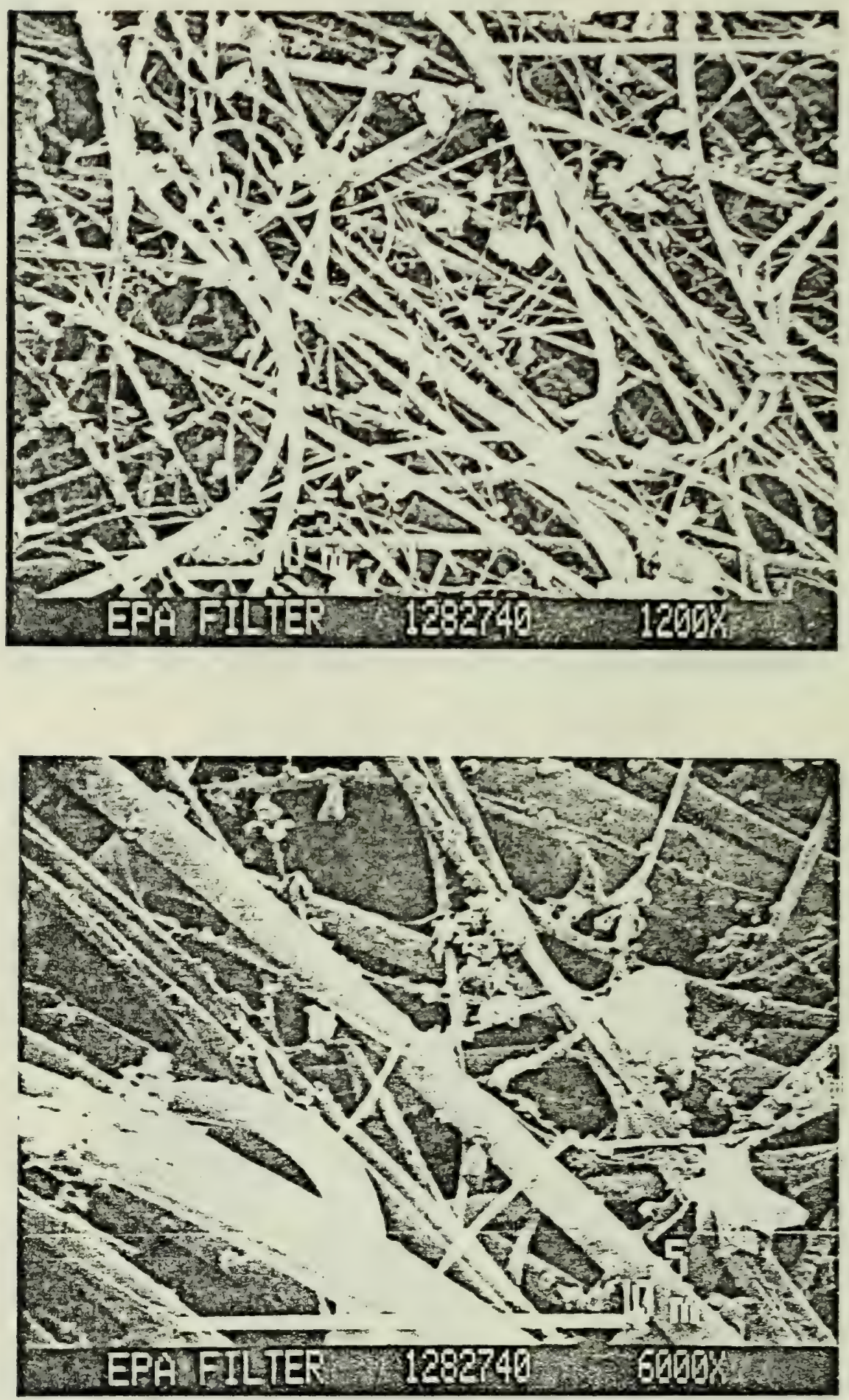
A. Site Identification

1. City: East Moline

Population: 21,000

2. Site Name and Address: John Deere Farts Bldg., 1400 13th St.

3. County: Rock Island Township: Hampton

4. USGS Topographical Map Name and Scale: Silvis, Iowa-IIl, $1: 24,000$

5. Site Elevation (Feet): 580

B. Site Classification/Representativeness

1. Dominating Influence on Site: Industrial, Commercial

C. Source Impact

1. Stationary Sources that may Influence Site:

\begin{tabular}{|c|c|c|c|c|c|}
\hline Name of Source & Location & $\begin{array}{c}\text { Direction } \\
\text { From } \\
\text { Site } \\
\end{array}$ & $\begin{array}{l}\text { Distance } \\
\text { From } \\
\text { Site } \\
\end{array}$ & Pollutant & $\begin{array}{l}\text { Emissions } \\
\text { (Tons/Yr) } \\
\end{array}$ \\
\hline International Harvester & $\begin{array}{l}1100 \text { 3rd St. } \\
\text { E. Moline }\end{array}$ & WNW & $1.7 \mathrm{~km}$ & $\begin{array}{l}\text { TSP } \\
H C\end{array}$ & $\begin{array}{l}135 \\
692\end{array}$ \\
\hline Deere-Harvester & $\begin{array}{l}1100 \text { 13th Ave. } \\
\text { E. Moline }\end{array}$ & NW & $0.4 \mathrm{~km}$ & $\begin{array}{l}\mathrm{TSP} \\
\mathrm{SO}_{2} \\
\mathrm{NO}_{x} \\
\mathrm{HC}\end{array}$ & $\begin{array}{l}3,668 \\
2,720 \\
1,233 \\
1,027\end{array}$ \\
\hline Deere-Foundry & $\begin{array}{l}\text { Hwy. } 84 \text { \& 14th Ave. } \\
\text { E. Moline }\end{array}$ & ENE & $1.1 \mathrm{~km}$ & TSP & 27,053 \\
\hline Deere-Planter & $\begin{array}{l}501 \text { 3rd Ave. } \\
\text { Moline }\end{array}$ & w & $8.6 \mathrm{~km}$ & $\begin{array}{l}\text { TSP } \\
\text { HC }\end{array}$ & $\begin{array}{l}1,800 \\
1,956\end{array}$ \\
\hline Strombeck Mfg. & $\begin{array}{l}51 \text { st \& } 4 \text { th Ave. } \\
\text { Moline }\end{array}$ & WSW & $3.0 \mathrm{~km}$ & TSP & 569 \\
\hline
\end{tabular}


2. Mobile Sources that may Influence the Site:

$\begin{array}{lc}\text { Names of Roadways: } & \text { 13th Street } \\ \text { Type: } & \text { Local Street } \\ \begin{array}{l}\text { Distance of Roadway } \\ \text { from Site (ft) }\end{array} & 90 \\ \text { Composition of Roadway } & \text { Concrete } \\ \text { Number of Traffic Lanes } & 2 \\ \text { Average Daily Traffic } & 5000 \\ \text { Average Vehicle Speed (mph) } & 25 \\ \text { One or Two Way Traffic } & \text { Two } \\ \text { Number of Parking Lanes } & \text { None } \\ \text { Are Parking Lanes Used } & \\ \text { For Traffic Part of Day? } & \text { N/A } \\ \text { Is Dust Visibiity Retained } & \text { No } \\ \text { Does Roadway Have Curb } & \text { Yes }\end{array}$

3. Area Sources that may Influence the Site:

$\begin{array}{llcc}\text { Type of Source } & \begin{array}{c}\text { Direction } \\ \text { From Site }\end{array} & \begin{array}{c}\text { Distance } \\ \text { From Site }\end{array} & \text { Pollutant } \\ \text { Silvis Railyard } & \text { ESE } & 1 \mathrm{~km} & \text { TSP } \\ \text { D. Topography/Obstructions } & & \end{array}$

1. General Characteristics Over a 2 Mile Radius From the Site: Rolling

2. Topographic Features that Influence the Site: (Types - hills, valleys, depressions, bodies of water, ridges, cliffs)

$\begin{array}{lccc}\text { Type } & \text { Size } & \begin{array}{l}\text { Direction } \\ \text { From Site }\end{array} & \begin{array}{l}\text { Distance } \\ \text { From Site }\end{array} \\ \text { Mississippi Bluffs } & 30 \mathrm{~m} \text { high } & \text { South } & 0.2 \mathrm{~km}\end{array}$

3. Obstructions to Wind Flow

(Types - buildings, trees, ridges, cliffs)

\begin{tabular}{llll} 
Type & Size & Direction & Distance \\
\hline
\end{tabular}

None 
E. Distance to the nearest National Weather Service (NWS) Site.

Here are the distances and directions to the nearest NWS observing site from the inhalable particulate monitor in East Moline.

Distances are in statute miles; directions are degrees from true north.

Site Address

1400 S. 13 th St.
Nearest NWS Site

Quad Cities Airport (MLI)
Directions \& Distance to NWS 


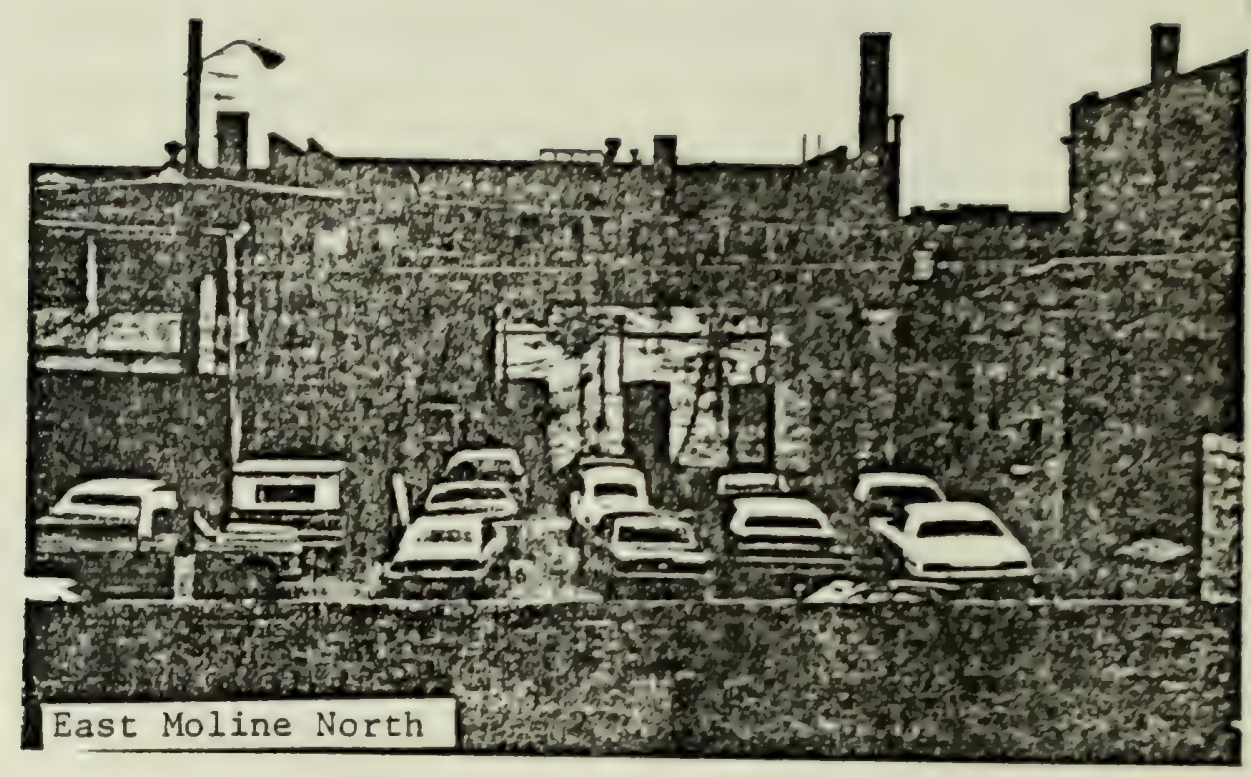

East Moline - North

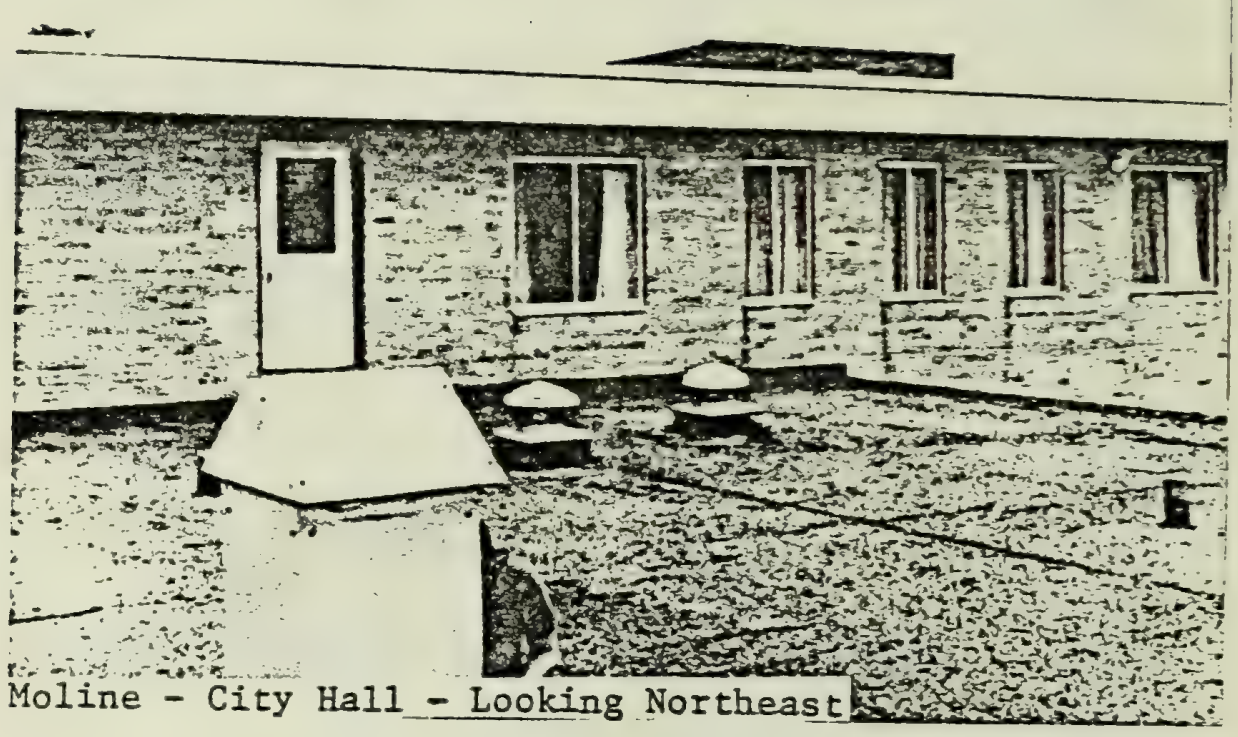

East Moline - North East 


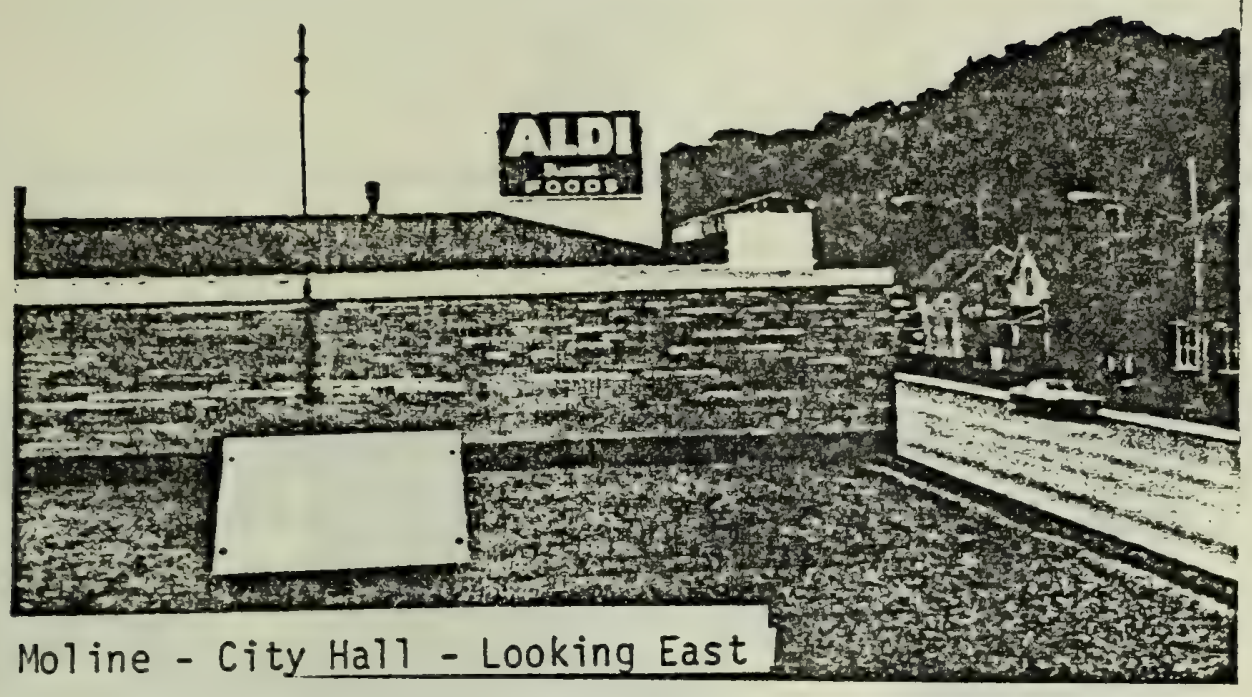

East Moline - East

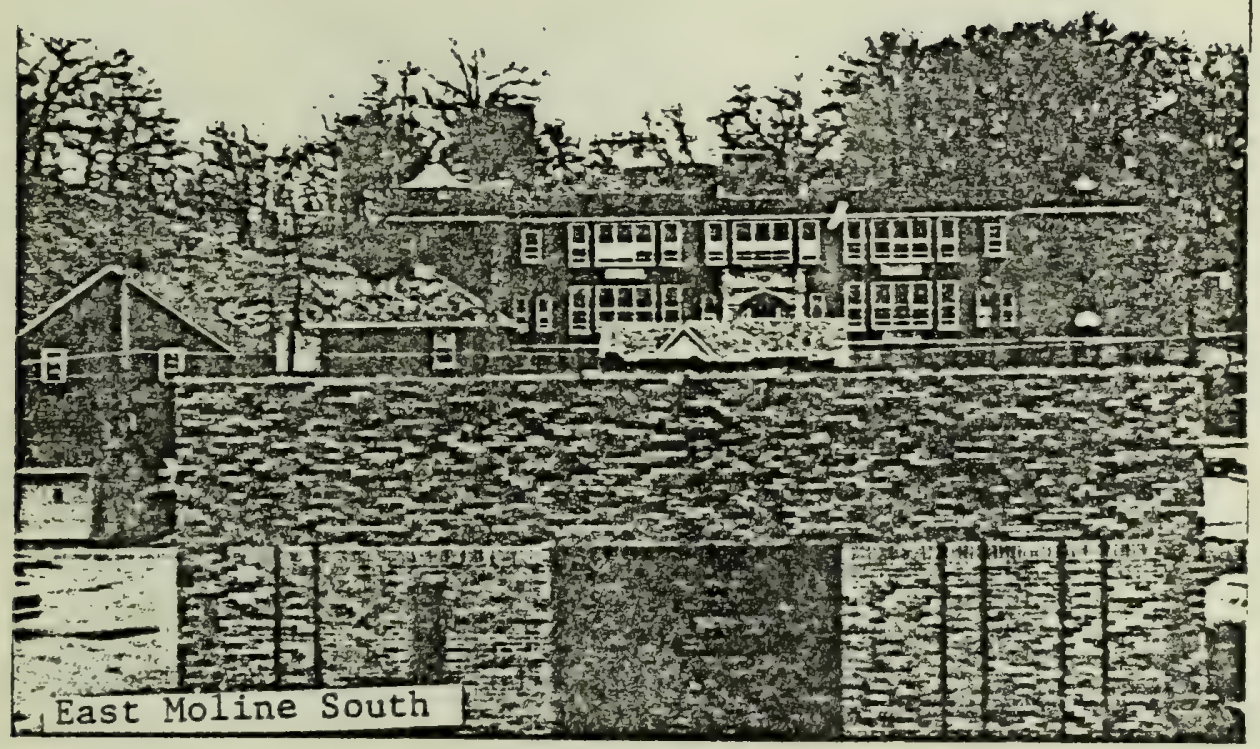

East Moline - South 


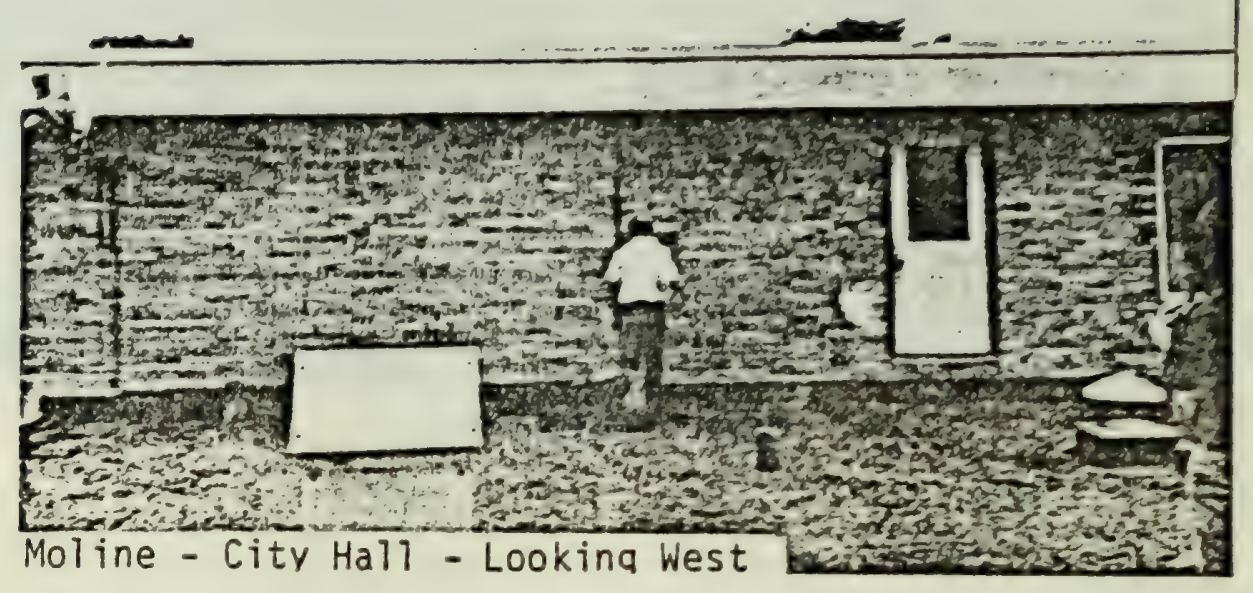

East Moline - West 

Particulate:
Minerals 99.5 Biological 1
Combustion
6.4
Other
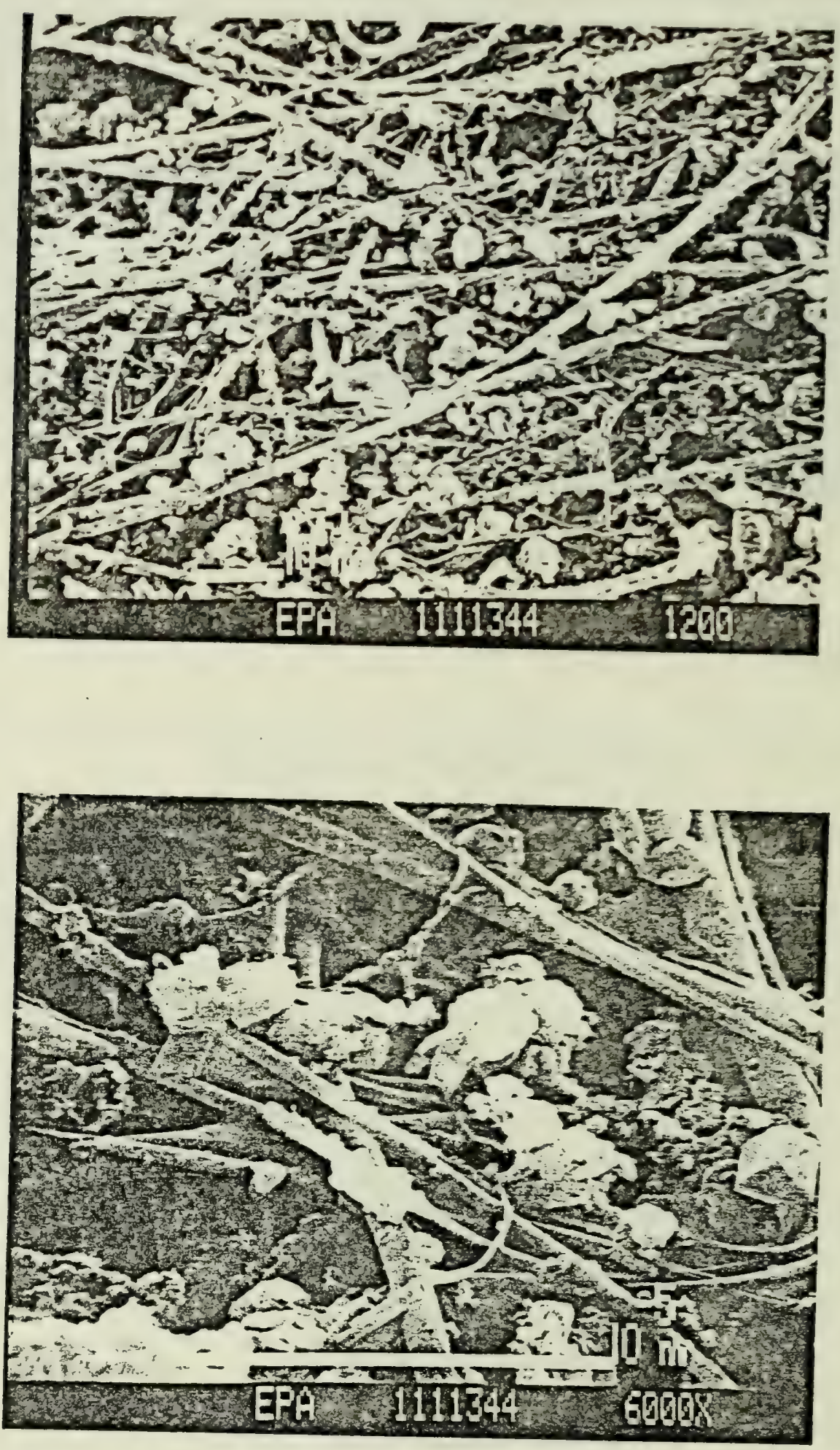


\begin{tabular}{cccc} 
Inhalable Particulate & \multicolumn{1}{c}{$96 / \mathrm{m}^{3}$} & \\
Particulate: Minerals 92.2 & Combustion & 3.8 \\
Biological 1 & Other & - \\
Low temperature ashing, $\%$ loss & 25.5
\end{tabular}
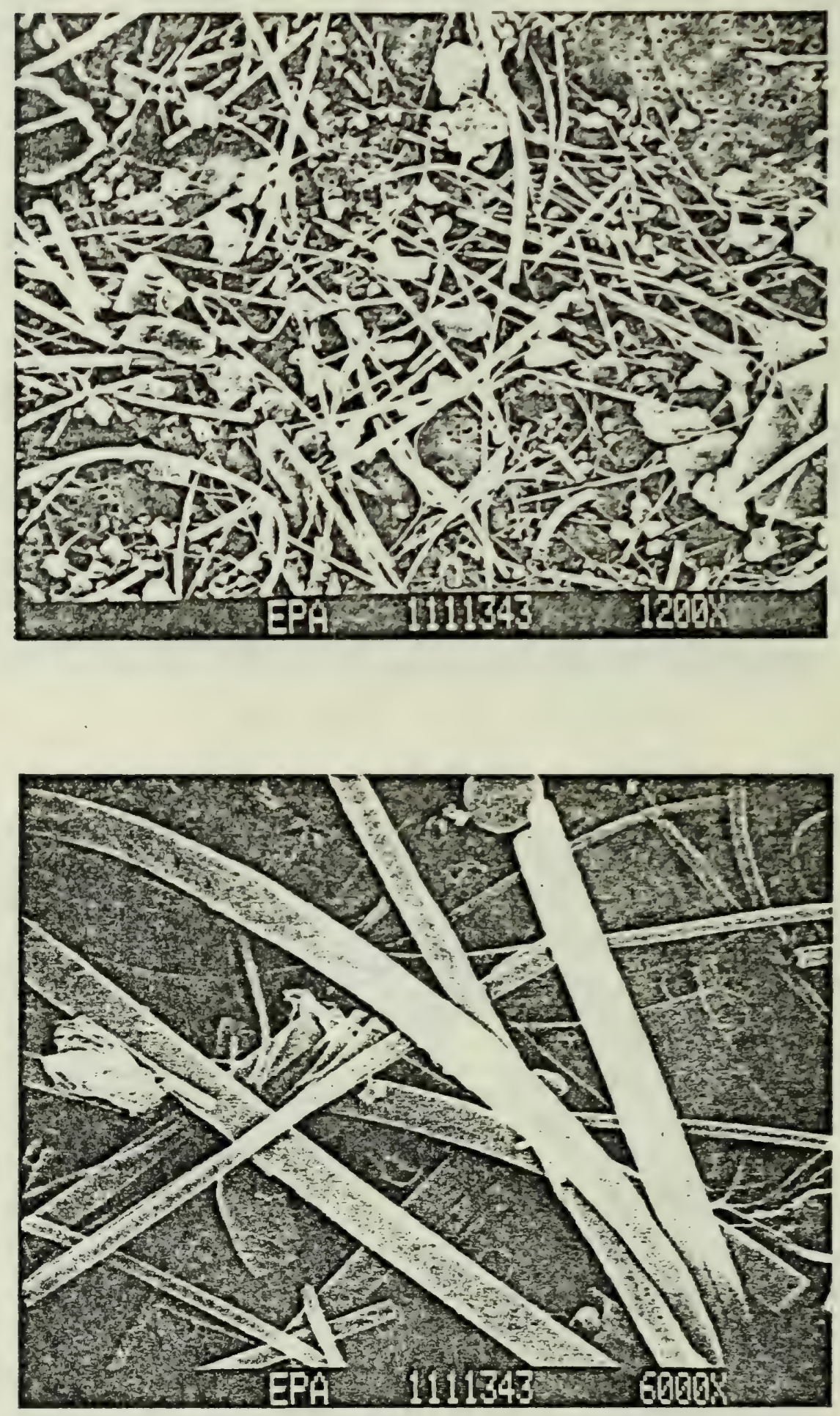


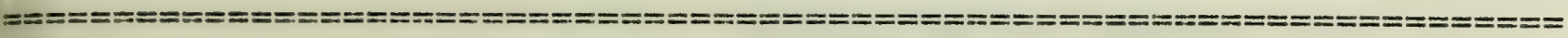
L.TER NLIMEER : 1230045

NETWOFK :

SITE : E. MOL.TNE/J. LIEEFE

LLEE.TION DATE: : 06.103/81

DE.JEI:TIVE MAR. : 25

COMFINENT

AVEFIATIE

F'ART. SIZE
FAMIIEE

(1)

WEI IIHT

PERCENT
Number

Percent

\section{MINERALS}

QUAFTZIFELDSFAFIS

LIMESTONE

IFION DXIDES:

OTHER MINEFALS
17. 3

13. 1

1.1. 1

13. 8

SIOTT

GLASSY FLYASH SFHEFES

BIOLLGITAL

PUILLEN \& SFIIFES

PLANT TISEIJE

STAFI:H
11. 1

1E. 1

17. 4
5. $1-40.7$

1. $8-40.7$

5. $1-20.4$

5. $1-29.8$

5. $1-20.4$

10. $2-28.9$

14. $4-20.4$

$\begin{array}{rr}36 & 9 \\ 46 & 77 \\ 4 & 2 \\ 0 & 0\end{array}$

$5 \quad 8$

00

$2 \quad 2$

42

11

MISIELLLANEDUS:

FEFRIILIS METAL

FUIEEER

FARTILLE SIZE DISTFIEUTION

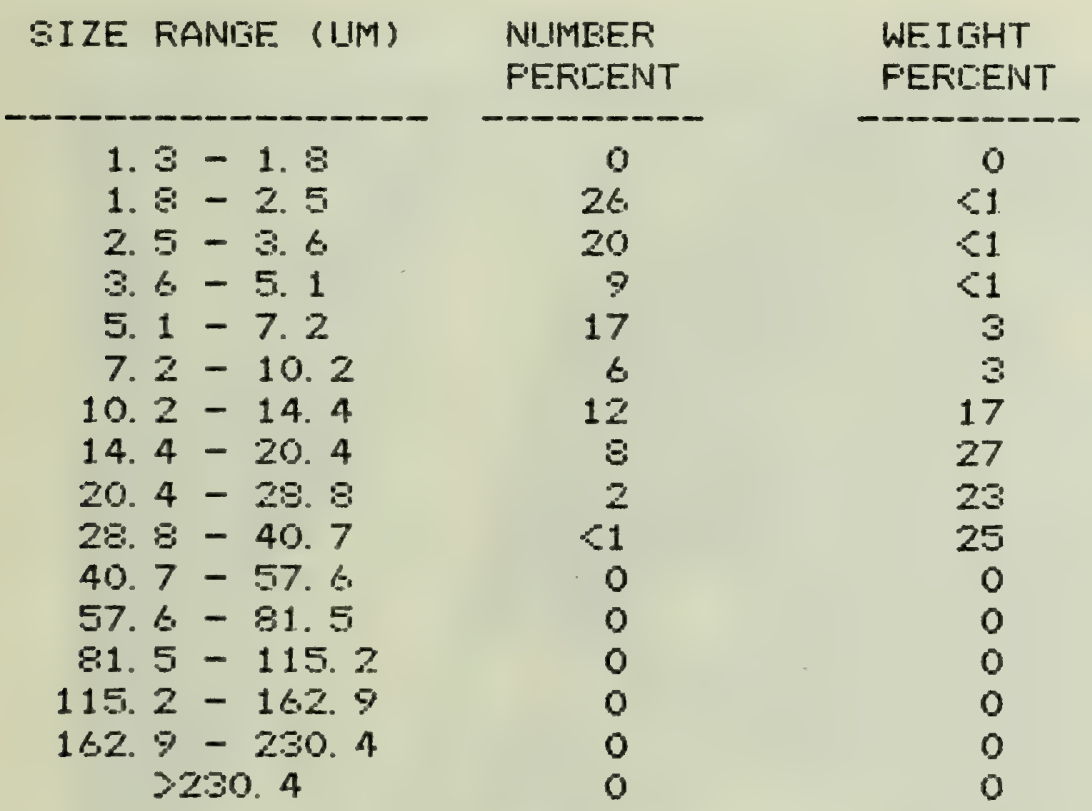


Total Suspended Particulate $\mu g / \mathrm{m}^{3}$

$\begin{array}{cccc}\text { Particulate: Minerals } 73 & \text { Combustion } & 4.2 \\ \text { Biological } 5.8 & \text { Other } & - \\ \text { Low temperature ashing, } \% \text { loss } & & 10.6\end{array}$
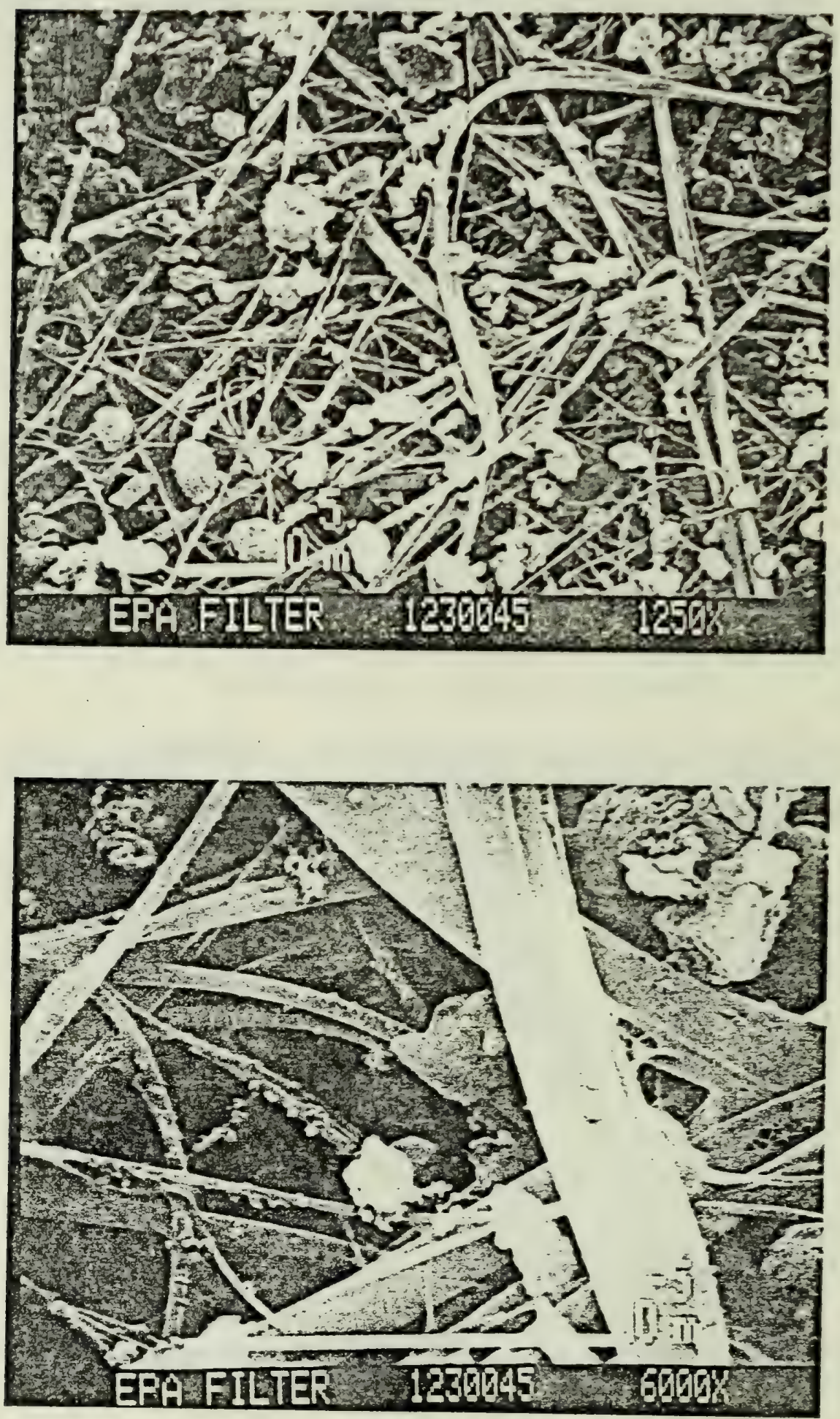


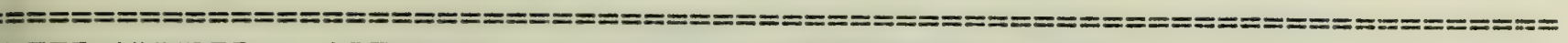

LTER NIMMEER : $127 \$ 669$

NETWIORK :

SITE : $\quad$ E. MOLINE/U. DEEFE

LLLELTION DATE : 06/03/81

DE.JEETIVE MAL. : Z ZS

\begin{tabular}{|c|c|c|c|c|}
\hline COMMFINENT & $\begin{array}{l}\text { AVERAISE } \\
\text { PAFT. SIZE }\end{array}$ & FANIEE & $\begin{array}{l}\text { WEIIIHT } \\
\text { FEFICENT }\end{array}$ & $\begin{array}{l}\text { Number } \\
\text { Percent }\end{array}$ \\
\hline
\end{tabular}

\section{MINE.RAL.S}

QUIARTZ/FELDEFAFE:

LIMESTONE

IRIN OXIDES

OTHER MINEFIALS
8. 5

9. 8

5. 1

7. 7

SODT

GLASSY FLYASH SFHERES

BIOI..DISICAL

FUI.LEN \& SFIIFES

PILANT TISSUIE

STAFI:H

10. 5

1. $8-20.4$

1. $8-28.8$

24

6.5

1. $8-10.2$

$<1$

0

15

69

1

0

13

1. $8-20.4$

6

0

7. $2-14.4$

$\begin{array}{rr}2 & 2 \\ 0 & <1 \\ 0 & <1\end{array}$

MISTELLAIMEOUI:

FEFRIIUS METAL

RLIBEER

SUFT CIIAL.

12. 8

7. 2-20. 4

0

0

0
0
1

\section{FAFTICILE SIZE DIETRIEILITION}

\begin{tabular}{cc} 
SIZE FANIEE (HM) & NIIMEEF \\
FEEILENT \\
\hline $1.3-1.8$ & 0 \\
$1.5-2.5$ & 15 \\
$2.5-3.6$ & 19 \\
$3.6-5.1$ & 20 \\
$5.1-7.2$ & 23 \\
$7.2-10.2$ & 16 \\
$10.2-14.4$ & 5 \\
$14.4-20.4$ & 2 \\
$20.4-28.8$ & 61 \\
$28.8-40.7$ & 0 \\
$40.7-57.6$ & 0 \\
$57.6-81.5$ & 0 \\
$81.5-115.2$ & 0 \\
$115.2-162.9$ & 0 \\
$162.9-230.4$ & 0 \\
3230.4 & 0
\end{tabular}

WEIGHT

F'E.FI:ENT

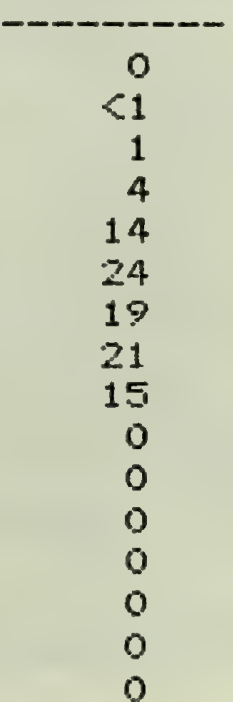


Inhalable Particulate $\quad \mu g / \mathrm{m}^{3} \quad 60$

$\begin{array}{ccll}\text { Particulate: Minerals } 54 & \text { Combustion } & 3.6 \\ \text { Biological } 1.2 & \text { Other } & 1.2 \\ \text { Low temperature ashing, \% loss } & & 0.0\end{array}$
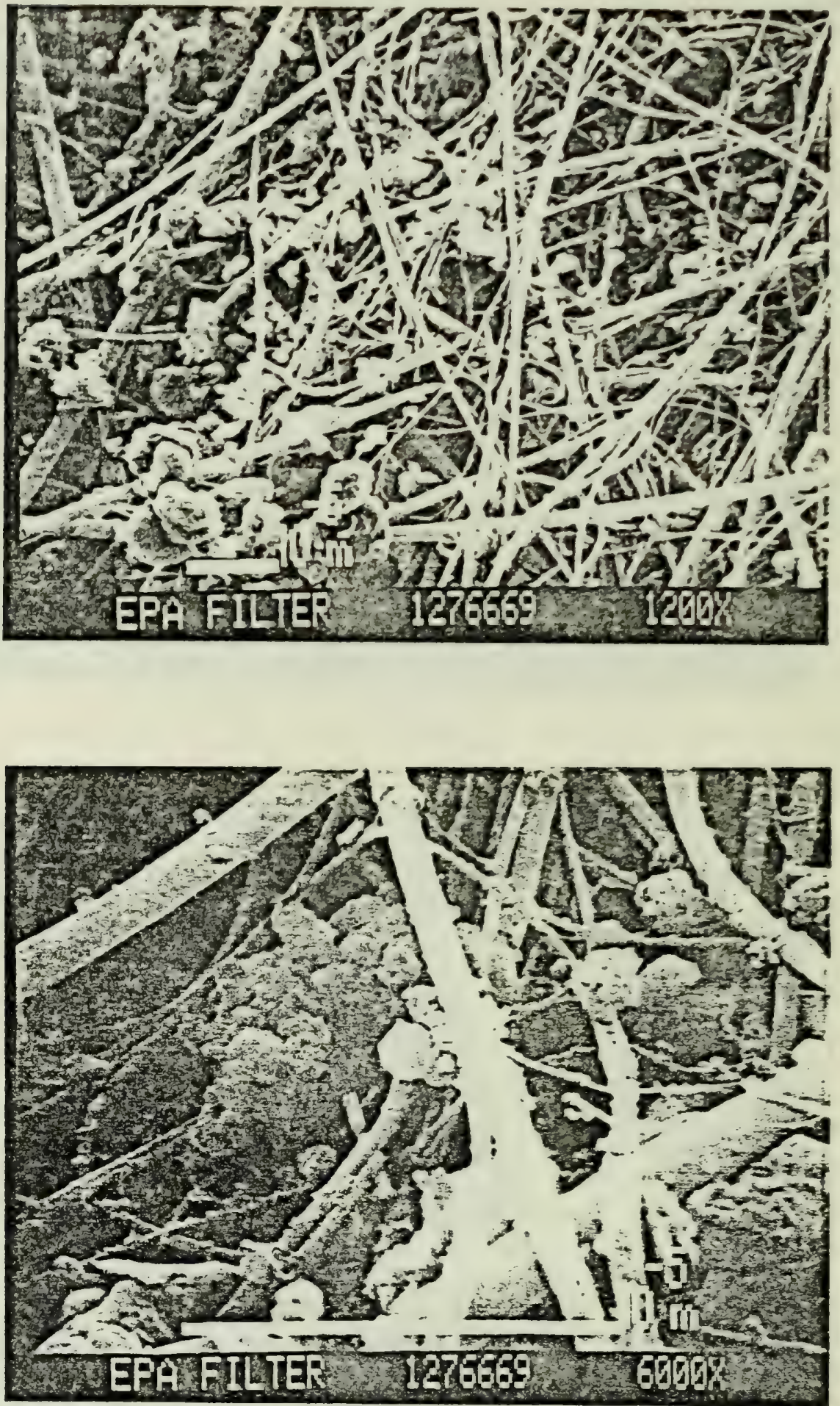


\section{TER NIIMPER : $128237 \%$}

LECTION DATE : $12 / 18 / 81$
NETWOFK :

SITE :

OE.JECTIVE MAT. : 25

\section{COMPONENT}

AVERTAGE

F'ART. SI ZE
RANISE.

13.9
13.9
5.6

13. 0

3. $6-29.8$

2.. $5-40.7$

2. $5-40.7$

2. $5-10.2$

OTHER MINERALS

\section{COMEUSTION FRONURTE}

SDOT

GLASSY FLYASH SFHEFES

BIOI_OGICAL

FOLLEN \& SFORES

FLLANT TISSLIE

STARI:H
17. 0

3. $8-40.7$

$\begin{array}{rr}16 & 7 \\ 76 & 83 \\ 61 & 1 \\ 0 & 0\end{array}$

WEIGHT Number

FERTENT Percent

\section{ISCELL_ANFIOLS:}

FEFROLIS METAL

RIIEEER

$\begin{array}{ll}0 & 0 \\ 0 & 0\end{array}$

FARTIILLE SIZE DIETFIEUITIONN

\begin{tabular}{|c|c|c|}
\hline SIZE FAMIGE (UIM) & $\begin{array}{l}\text { NUIMEER } \\
\text { FEFICENT }\end{array}$ & $\begin{array}{l}\text { WEIGHT } \\
\text { FERICENT }\end{array}$ \\
\hline $1.3-1.8$ & 0 & 0 \\
\hline $1.8-2.5$ & 0 & 0 \\
\hline $2.5-3.6$ & 16 & $<1$ \\
\hline $3.6-5.1$ & 36 & 2 \\
\hline $5.1-7.2$ & 19 & 4 \\
\hline $7.2-10.2$ & 12 & 7 \\
\hline $10.2-14.4$ & 9 & 12 \\
\hline $14.4-20.4$ & 4 & 19 \\
\hline $20.4-28.8$ & 2 & 25 \\
\hline $28.8-40.7$ & $<1$ & 32 \\
\hline $40.7-57.6$ & 0 & 0 \\
\hline $57.6-81.5$ & 0 & 0 \\
\hline $81.5-115.2$ & 0 & 0 \\
\hline $115.2-182.9$ & 0 & 0 \\
\hline $162.9-230.4$ & 0 & 0 \\
\hline$>230.4$ & 0 & 0 \\
\hline
\end{tabular}




$\begin{array}{cclc}\text { Particulate: Minerals } & 53.4 & \text { Combustion } & 2.3 \\ \text { Biological } 2.3 & \text { Other } & - \\ \text { Low temperature ashing, } \% \text { loss } & & 0.0\end{array}$
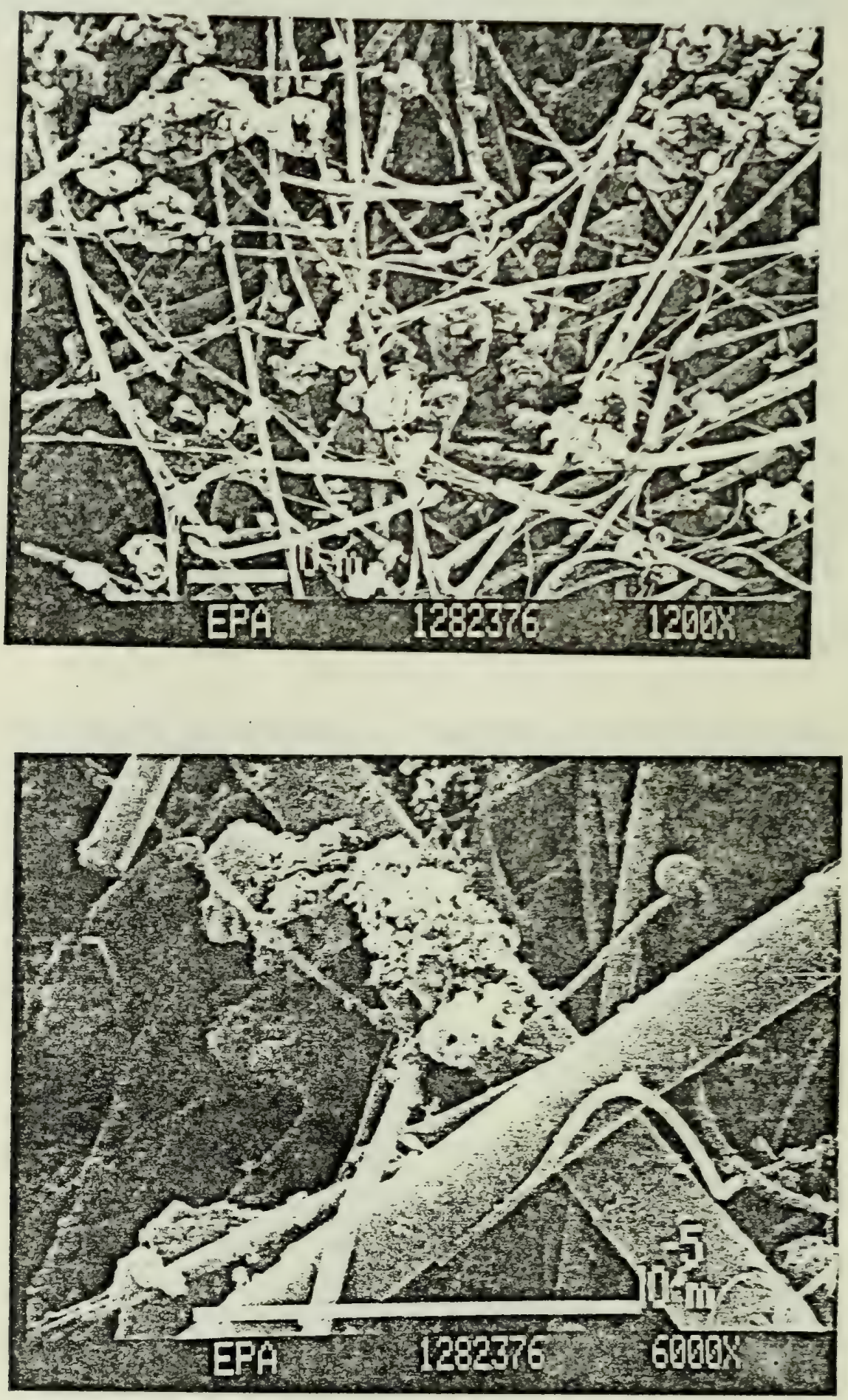


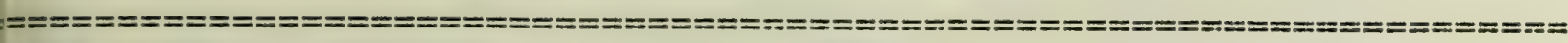
I.TER NLIMEER : 1282375 NETWIEK :
SITE :
E. MILL INE/J. TIEEFIE

I_LECTIIN DATE : $12 / 18 / 81$

DE.JECTIVE MAG. : 25

AVERAGE

FAFT. SIZE
3. $6-20.4$

2. $5-20.4$

5. $1-20.4$ FIANIEE WEI THTT WEI IIHT Number FEFIL:ENT Percent

MINERALS

QUIAFTZ/FEI_LISFAFIS

LIMESTINE.

IRUN OXIDES

OTHER MINERALS

9. 8

8. 7

$\begin{array}{rr}3 & 2 \\ 77 & 85 \\ 4 & 1 \\ 0 & 0\end{array}$

COMEILSTION PROTHIITS:

SODT

GI_ASSY FI_YASH SFHEFES

9.5

2. $5-20.4$

$4 \quad 9$

BIOLLOSICAL.

FUI_LEN \& SFIDFES

PLAANT TISEUIE

STAFICH

18. 1

19. 6

14. 9

10. $2-28.9$

7. $2-40.7$

10. $2-20.4$

0

0

MISIELLLANFOIIS

FEFFFIUS METAL_

RUEEER

FAFITIOLE SI DE DISTFIEITION

\begin{tabular}{cc} 
SIZE FAIVIE (UM) & NIIMEEF \\
FEEFENT \\
\hline $1.3-1.8$ & 0 \\
$1.3-2.5$ & 0 \\
$2.5-3.6$ & 16 \\
$3.6-5.1$ & 25 \\
$5.1-7.2$ & 24 \\
$7.2-10.2$ & 21 \\
$10.2-14.4$ & 11 \\
$14.4-20.4$ & 3 \\
$20.4-23.8$ & 61 \\
$28.8-40.7$ & 61 \\
$40.7-57.6$ & 0 \\
$57.6-81.5$ & 0 \\
$81.5-115.2$ & 0 \\
$115.2-162.9$ & 0 \\
$162.9-230.4$ & 0 \\
2230.4 & 0
\end{tabular}

WEIIIHT

FEFICENT

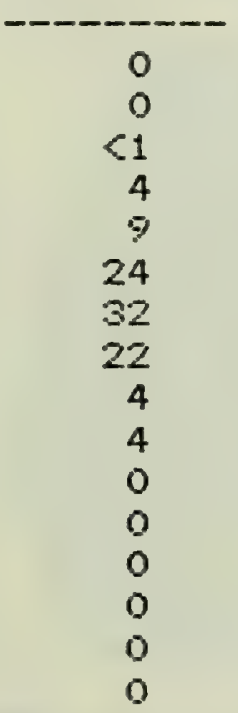


Inhalable Particulate $\quad \mu g / \mathrm{m}^{3}$

Particulate: Minerals 38.7 Combustion 1.8 Biological 6.0 Other

Low temperature ashing, \% loss
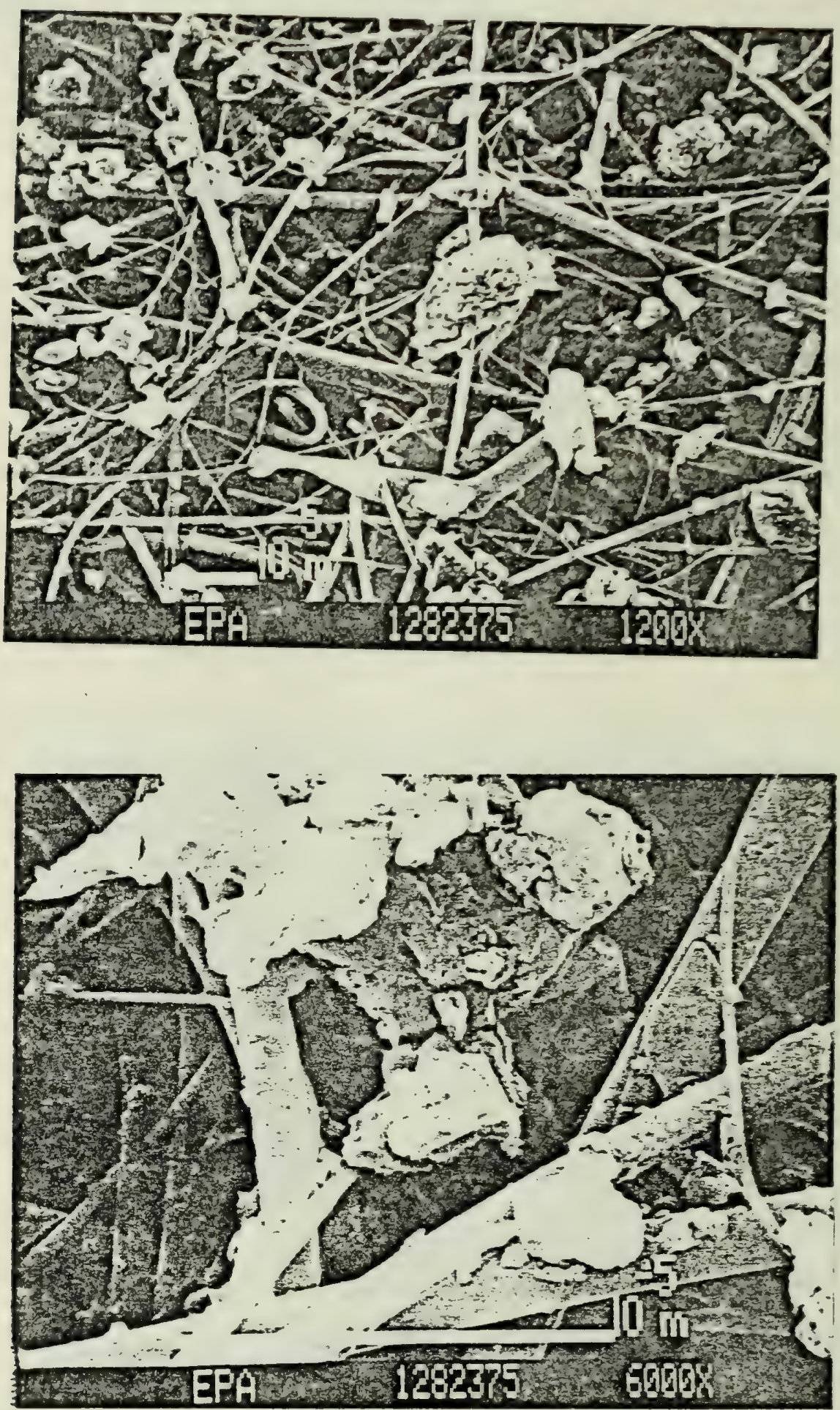
A. Site Identification

1. City: Peoria

2. Site Name and Address:State Welfare Office, 610 N.E. Jefferson

3. County: Peoria Township: Peoria

4. USGS Topographical Map Name and Scale: Peoria-East, 1:24000

5. Site Elevation (Feet): 510

B. Site Classification/Representativeness

1. Dominating Influence on Site: Commercial, Residential

C. Source Impact

1. Stationary Sources that may Influence Site:

\begin{tabular}{|c|c|c|c|c|c|}
\hline Name of Source & Location & $\begin{array}{c}\text { Direction } \\
\text { From } \\
\text { Site } \\
\end{array}$ & $\begin{array}{c}\text { Distance } \\
\text { From } \\
\text { Site } \\
\end{array}$ & Pollutant & $\begin{array}{l}\text { Emissions } \\
\text { (Tons } / Y r \text { ) }\end{array}$ \\
\hline ia Brick \& Tile & $\begin{array}{l}\text { Cole St. } \\
\text { E. Peoria }\end{array}$ & $S$ & $2.4 \mathrm{mi}$ & TSP & 664 \\
\hline - Wallace Sta. & $\begin{array}{l}1126 \text { W. Camp St } \\
\text { E. Peoria }\end{array}$ & t. $s$ & $.9 \mathrm{mi}$ & $\begin{array}{l}\mathrm{TSP} \\
\mathrm{SO}_{2} \\
\mathrm{NO}_{\mathrm{X}} \\
\mathrm{CO}^{2}\end{array}$ & $\begin{array}{r}5,661 \\
3,751 \\
2,348 \\
131\end{array}$ \\
\hline Coast Grain, & $\begin{array}{l}\text { Altoffer Lane } \\
\text { E. Peoria }\end{array}$ & S & $.9 \mathrm{mi}$ & TSP & 790 \\
\hline $1 t$ Assoc. Inc. & $\begin{array}{l}500 \text { W. Camp St. } \\
\text { E. Peoria }\end{array}$ & $S$ & $1.6 \mathrm{mi}$ & TSP & 7,875 \\
\hline Products & $\begin{array}{l}1300 \text { S. Second } \\
\text { St., Pekin }\end{array}$ & SSW & $10.4 \mathrm{mi}$ & $\begin{array}{l}\text { TSP } \\
\mathrm{SO}_{2} \\
\mathrm{NO}_{\mathrm{X}} \\
\mathrm{CO}\end{array}$ & $\begin{array}{r}265,338 \\
3,825 \\
1,893 \\
105\end{array}$ \\
\hline Powerton & $\begin{array}{l}\text { Box } 158 \\
\text { Pekin }\end{array}$ & SSW & $11.8 \mathrm{mi}$ & $\begin{array}{l}\mathrm{TSP} \\
\mathrm{SO}_{2} \\
\mathrm{NO}_{\mathrm{X}} \\
\mathrm{HC} \\
\mathrm{CO}\end{array}$ & $\begin{array}{r}188,235 \\
67,566 \\
136,221 \\
743 \\
2,482\end{array}$ \\
\hline
\end{tabular}




\begin{tabular}{|c|c|c|c|c|}
\hline $\begin{array}{l}\text { Caterpillar Tractor } \\
\text { Co. }\end{array}$ & $\begin{array}{l}2400 \text { S.W. SW } \\
\text { Washington, Peoria }\end{array}$ & $2.1 \mathrm{mi}$ & $\begin{array}{l}\text { TSP } \\
H C\end{array}$ & $\begin{array}{l}714 \\
115\end{array}$ \\
\hline ADM Riverside & $\begin{array}{l}\text { Edmund St. } \\
\text { Peoria }\end{array}$ & $1.6 \mathrm{mi}$ & TSP & 3,743 \\
\hline $\begin{array}{l}\text { Keystone Steel \& } \\
\text { Wire Div. }\end{array}$ & $\begin{array}{l}2000 \text { S. Adams } \\
\text { St., Peoria }\end{array}$ & $4.9 \mathrm{mi}$ & $\begin{array}{l}\text { TSP } \\
\text { NOx }\end{array}$ & $\begin{array}{r}3,902 \\
458\end{array}$ \\
\hline Peoria Blacktop & $\begin{array}{l}3300 \text { W. SouthportwNW } \\
\text { Rd., Peoria }\end{array}$ & $4.2 \mathrm{mi}$ & TSP & 2,960 \\
\hline Bemis Co. Inc. & $\begin{array}{l}\text { Ft. of sloan St. NE } \\
\text { Peoria }\end{array}$ & $1.5 \mathrm{mi}$ & $\begin{array}{l}\mathrm{TSP} \\
\mathrm{SO} 2 \\
\mathrm{NO}{ }_{x}\end{array}$ & $\begin{array}{r}1,639 \\
1,301 \\
381\end{array}$ \\
\hline Celotex Corp & $\begin{array}{l}2226 \text { W. Clarke } \\
\text { St., Peoria }\end{array}$ & $4.1 \mathrm{mi}$ & $\begin{array}{l}\mathrm{TSP} \\
\mathrm{SO}_{2} \\
\mathrm{NO}_{x}\end{array}$ & $\begin{array}{r}5,392 \\
2,307 \\
794\end{array}$ \\
\hline $\begin{array}{l}\text { George E. Hoffman } \\
\text { \& Sons }\end{array}$ & $\begin{array}{l}94 \text { S. Sanger St. SW } \\
\text { Peoria }\end{array}$ & $2.5 \mathrm{mi}$ & TSP & 1,834 \\
\hline $\begin{array}{l}\text { United Ready Mix, } \\
\text { Inc. }\end{array}$ & $\begin{array}{l}\text { Ft. of Green St. SE } \\
\text { Peoria }\end{array}$ & $.2 \mathrm{mi}$ & TSP & 134 \\
\hline Wahl feld Mfg. Co. & $\begin{array}{l}1100 \text { SW WashingtonSW } \\
\text { Peoria. }\end{array}$ & $1.4 \mathrm{mi}$ & TSP & 156 \\
\hline Hiram Walker \& Son & $\begin{array}{l}\text { Ft. of Edmund } \\
\text { Peoria }\end{array}$ & $1.6 \mathrm{mi}$ & $\begin{array}{l}\text { TSP } \\
\text { NOXX } \\
\text { HC }\end{array}$ & $\begin{array}{r}45,453 \\
112 \\
2,979\end{array}$ \\
\hline Pabst Brewing Co. & $\begin{array}{l}709 \text { N.E. Water St. S } \\
\text { Peoria }\end{array}$ & $.3 \mathrm{mi}$ & $\begin{array}{l}\mathrm{TSP} \\
\mathrm{SO}_{2}\end{array}$ & $\begin{array}{r}1,662 \\
146\end{array}$ \\
\hline Seneca Petroleum Co. & $\begin{array}{l}11 \text { Sanger St. } \\
\text { Peoria }\end{array}$ & $2.9 \mathrm{mi}$ & TSP & 1,176 \\
\hline Allied Mills Inc. & $\begin{array}{l}7501 \text { S. Adams SW } \\
\text { St., Bartonville }\end{array}$ & $5.7 \mathrm{mi}$ & TSP & 407,969 \\
\hline CILCO Edwards & Rural Bartonvillesw & $8.1 \mathrm{mi}$ & $\begin{array}{l}\mathrm{TSP} \\
\mathrm{SO}_{2} \\
\mathrm{NO}_{\mathrm{X}} \\
\mathrm{HC}\end{array}$ & $\begin{array}{r}169,975 \\
28,276 \\
17,239 \\
958\end{array}$ \\
\hline Pabst Brewing Co. & $\begin{array}{l}4541 \text { N. Prospect N } \\
\text { Peoria Heights }\end{array}$ & $3.2 \mathrm{mi}$ & $\begin{array}{l}\text { TSP } \\
\mathrm{NO}_{\mathrm{X}}\end{array}$ & $\begin{array}{r}29,451 \\
194\end{array}$ \\
\hline Peoria Barge Terminal & $\begin{array}{l}1925 \text { Darst St. SW } \\
\text { St., Peoria }\end{array}$ & $3.2 \mathrm{mi}$ & TSP & 265 \\
\hline
\end{tabular}


ork Foundry Supply o. aterpillar

aterpillar Tractor Route 24 Mapieton

$600 \mathrm{~W}$. WashingtonsSW East Peoria

2424 W. Clarke SW St., Peoria

$3.9 \mathrm{mi} \quad$ TSP

$1.6 \mathrm{mi}$

TSP

$\mathrm{SO}_{2}$

$\mathrm{NO}_{\mathrm{X}}$

$\mathrm{HC}$
$\mathrm{CO}$

SW

Mapleton

$12.2 \mathrm{mi}$

TSP

$\mathrm{SO}_{2}$

NOX

$\mathrm{HC}$

$3.8 \mathrm{mi}$ TSP

2411 W. Clarke SW St., Peoria
2,057

5,108

6,959

929

567

131

243,324

8,858

1,094

3,565

1,664

2. Mobile Sources that may Influence the Site:

Names of Roadways: Jefferson Adams Hancock Green

Type: Arterial Street Arterial Local Local

Distance of Roadway

$\begin{array}{lllll}\text { from Site (ft) } & 100 & 290 & 220 & 165\end{array}$

Composition of Roadway Asphalt Asphalt Asphalt Asphalt

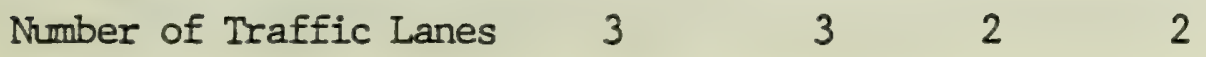

Average Daily Traffic $\quad \begin{array}{llll}1560 & 15500 \quad 1000 & 1000\end{array}$

Average Vehicle Speed (mph) $35 \quad 35 \quad 25-30 \quad 25-30$

One or Two Way Traffic One One Two Two

Number of Parking Lanes - Two None None None

Are Parking Lanes Used

For Traffic Part of Day? No

N/A N/A N/A

Is Dust Visibiity Retained No

No No No

Does Roadway Have Curb Yes Yes Yes Yes 
3. Area Sources that may Influence the Site:

Type of Source

Parking lot (gravel)

Parking lot

(cinder \& gravel)

Alley (asphalt \& gravel)

Parking lot

(cement \& gravel)

Rail yards :-

Parking lot

Vacant lot \begin{tabular}{l} 
Direction \\
from \\
Site \\
\hline
\end{tabular}

NW

NE

SE

SE \& S

$S E-E$

$\mathrm{SW}$

SE
Distance

from

Site

$153^{\prime}$

$93^{\prime}$

$65^{\prime}$

Directly by building

$.5 \mathrm{mi}$

$180^{\prime}$

$90^{\prime}$
TSP

TSP

Pollutant

TSP

TSP

TSP

TSP

TSP

D. Topography/Obstructions

1. General Characteristics Over a 2 Mile Radius From the Site: Rolling

2. Topographic Features that Influence the Site: (Types - hills, valleys, depressions, bodies of water, ridges, cliffs)

Type

River

Peoria Lake

River Valley

Hill
Size

.3 mi wide

$1.7 \mathrm{mi}$ across

Elevation: $450^{\prime}$

$600^{\prime}$ elevation
Direction

From Site

$\mathrm{s}$

E

S, E

WNW
Distance

From Site

$.5 \mathrm{mi}$

$.5 \mathrm{mi}$

Sloping from 510'

$450^{\prime}$ within .5 mi

$.8 \mathrm{mi}$ 
3. Obstructions to Wind Flow

(Types - buildings, trees, ridges, cliffs)

Type

$$
\text { Direction }
$$

Distance

$\underline{\text { Size }}$

From Site

From Site

None

4. Comments

Site (ele. 510') located $N+N W$ of river valley (ele. 450'). Approximately $8 / 10 \mathrm{mi}$ to the $\mathrm{WWW}$, the elevation rises to $600^{\prime}$.

E. Distance to the nearest National Weather Service (NWS) Site.

Here are the distances and directions to the nearest NWS observing site from the inhalable particulate monitor in Peoria. Distances are in statute miles; directions are degrees from true north.

Site Address

$610 \mathrm{NE}$ Jefferson
Nearest NWS Site

Peoria Airport (PIA)
Directions and Distance to NWS $245^{\circ}$ at $5.6 \mathrm{mi}$. (WSW) 


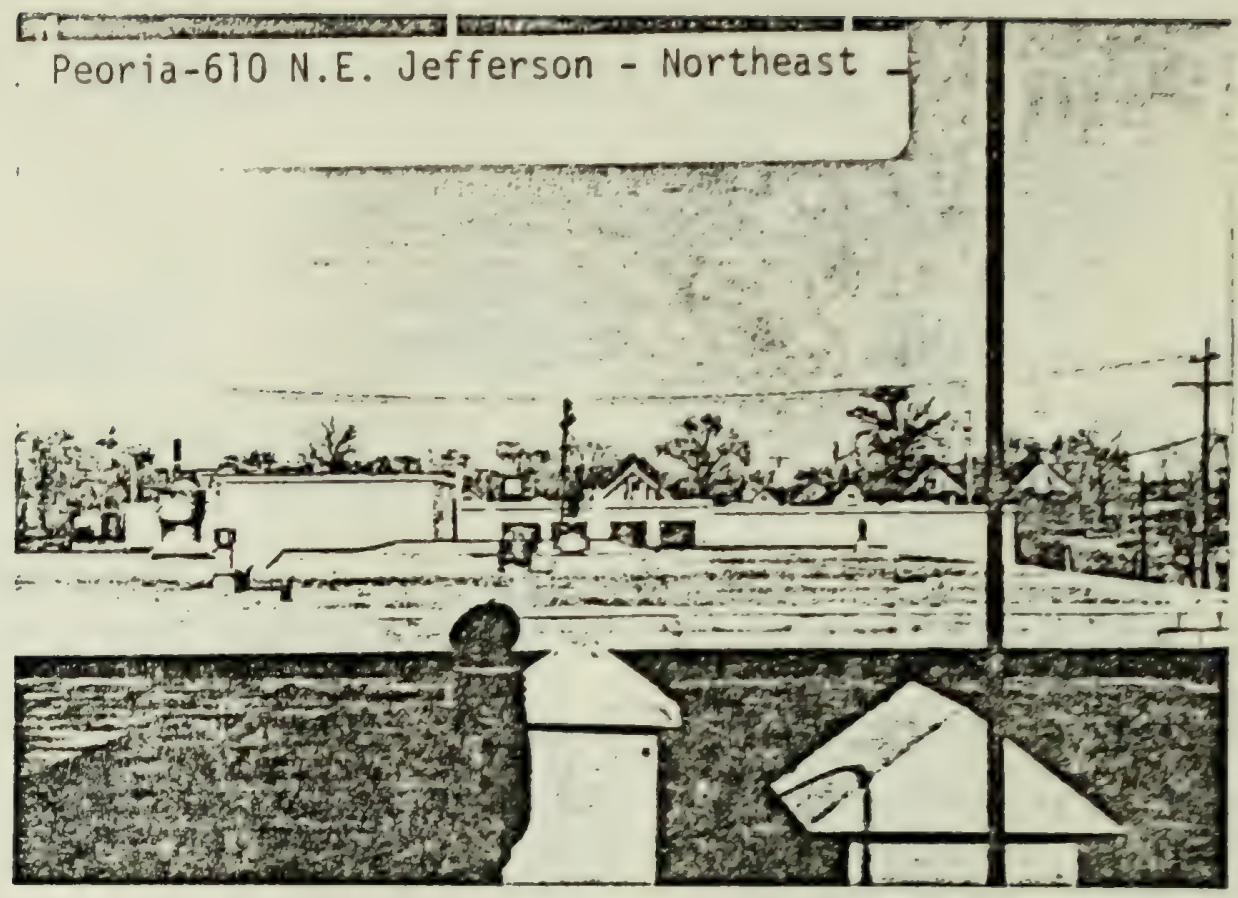

Peoria - North East

- A 36 -

Peoria-610 N.E. Jefferson - Southeast -

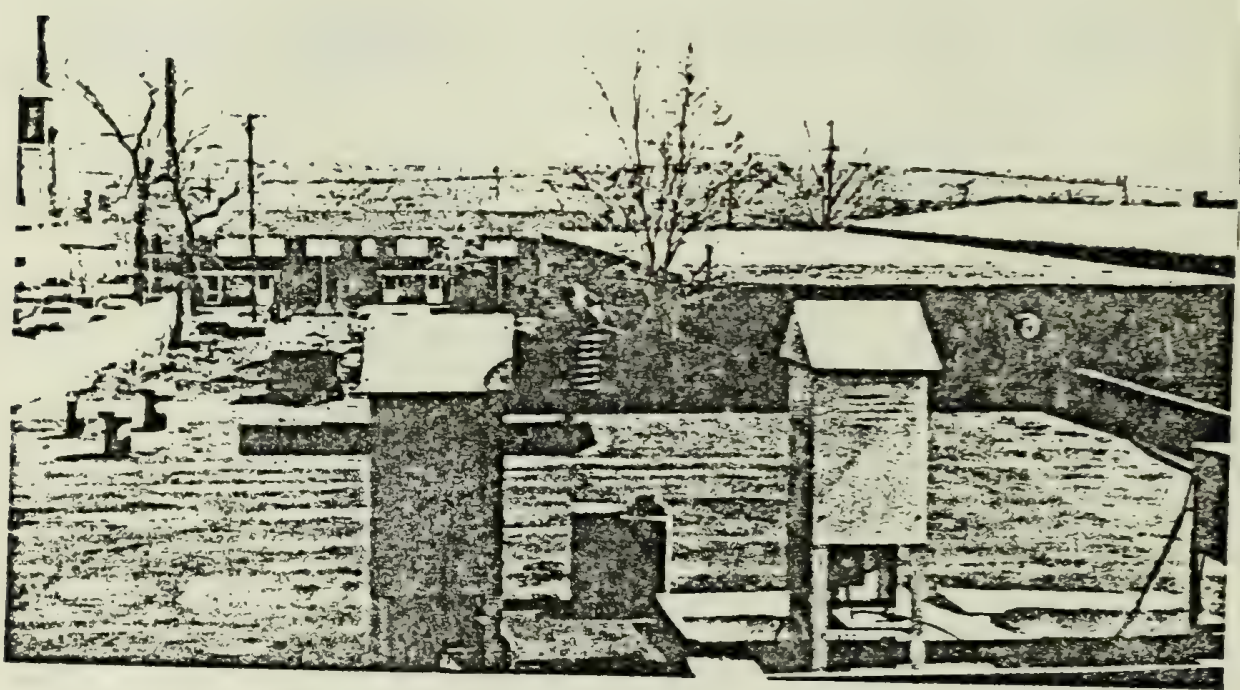

Peoria - South East 


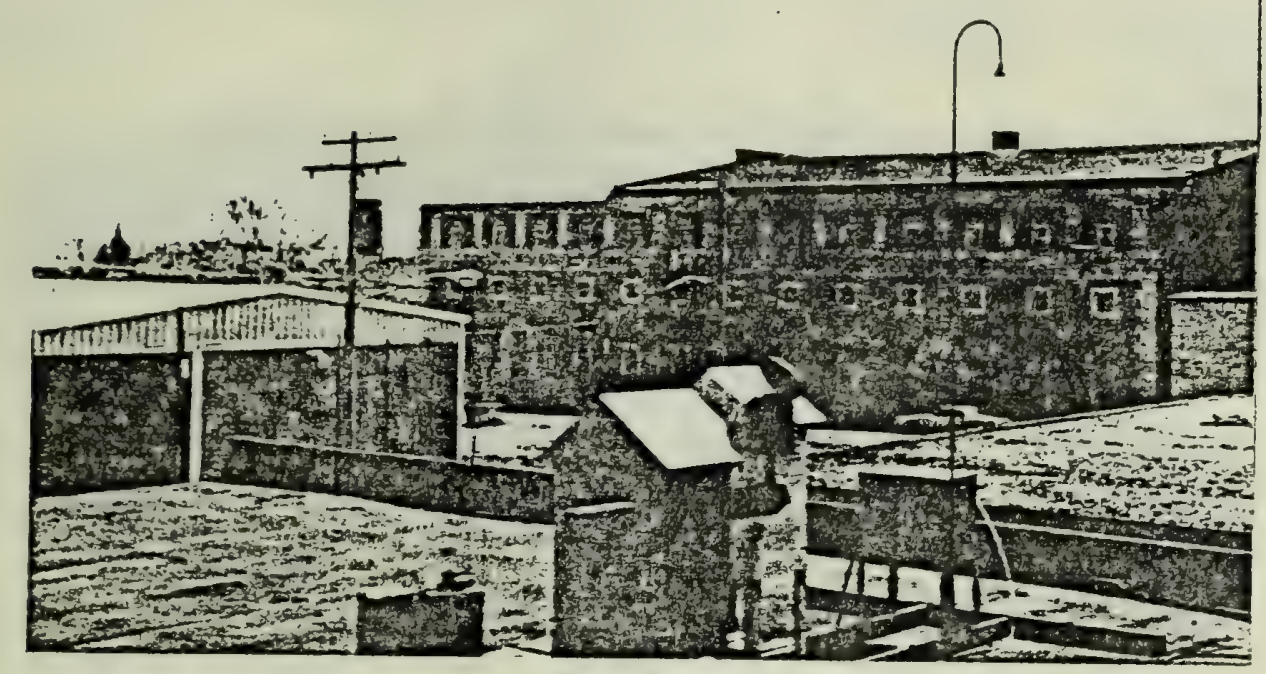

Peoria - South

Peoria-610 N.E. Jefferson - Southwest

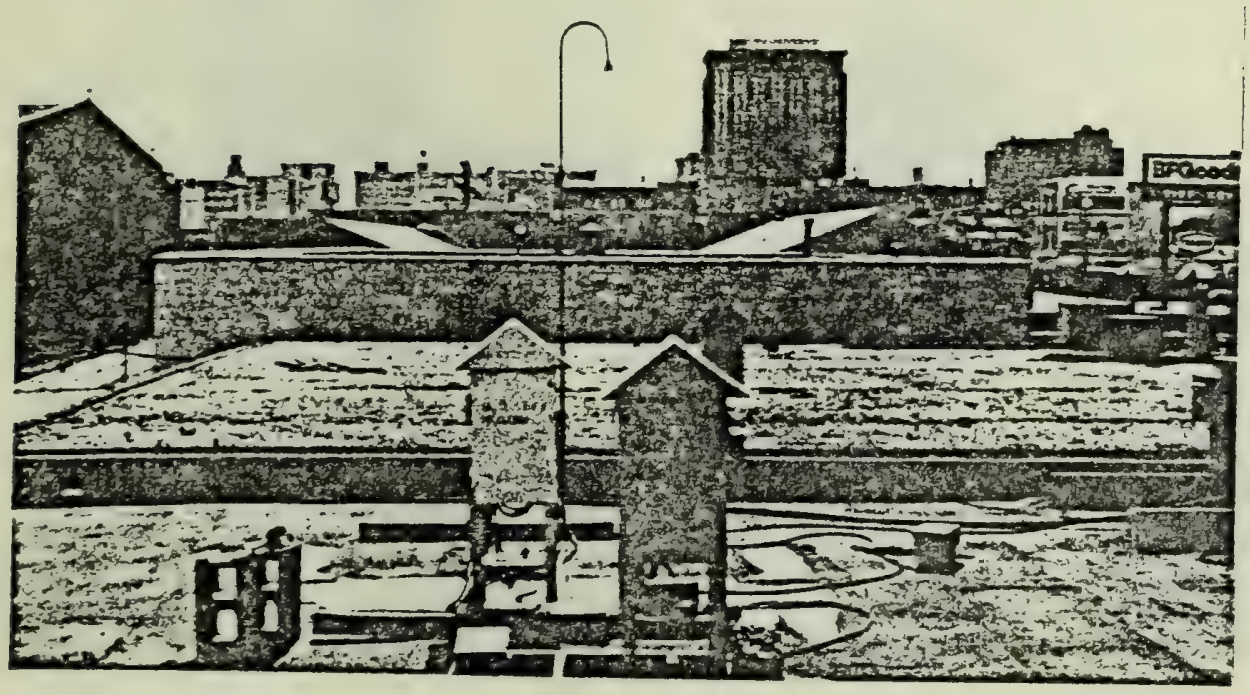

Peoria - South West 


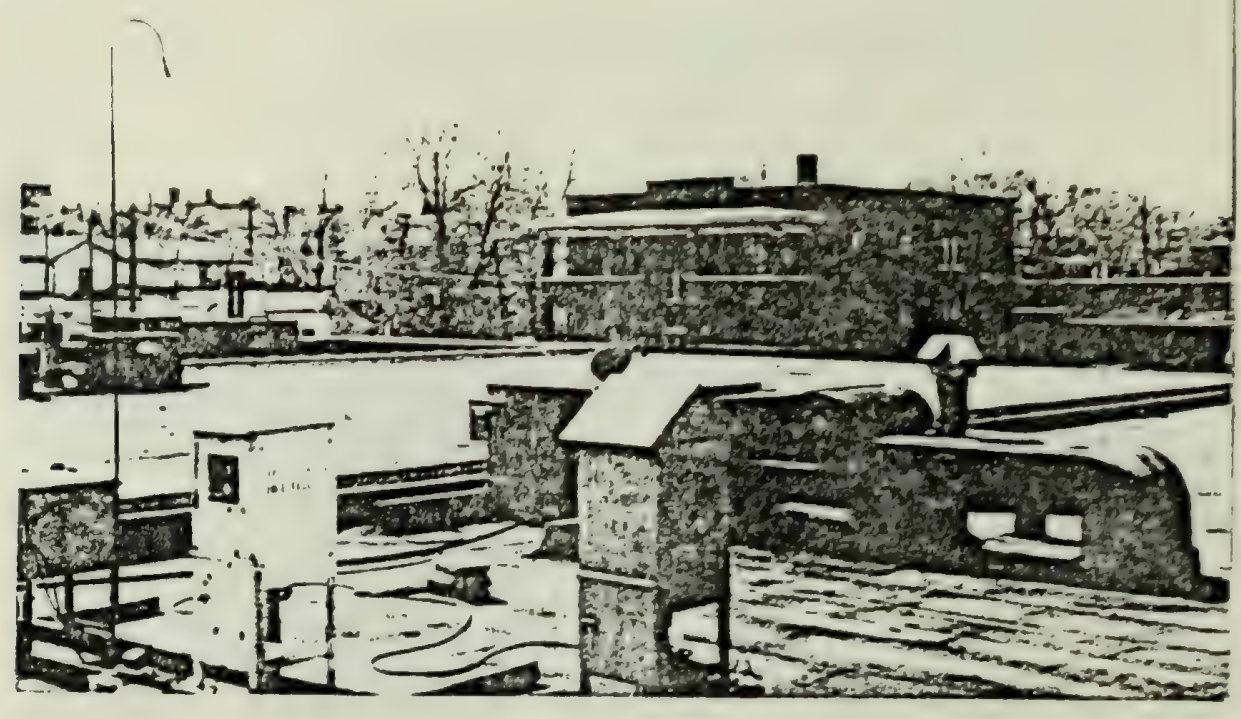

Peoria - West

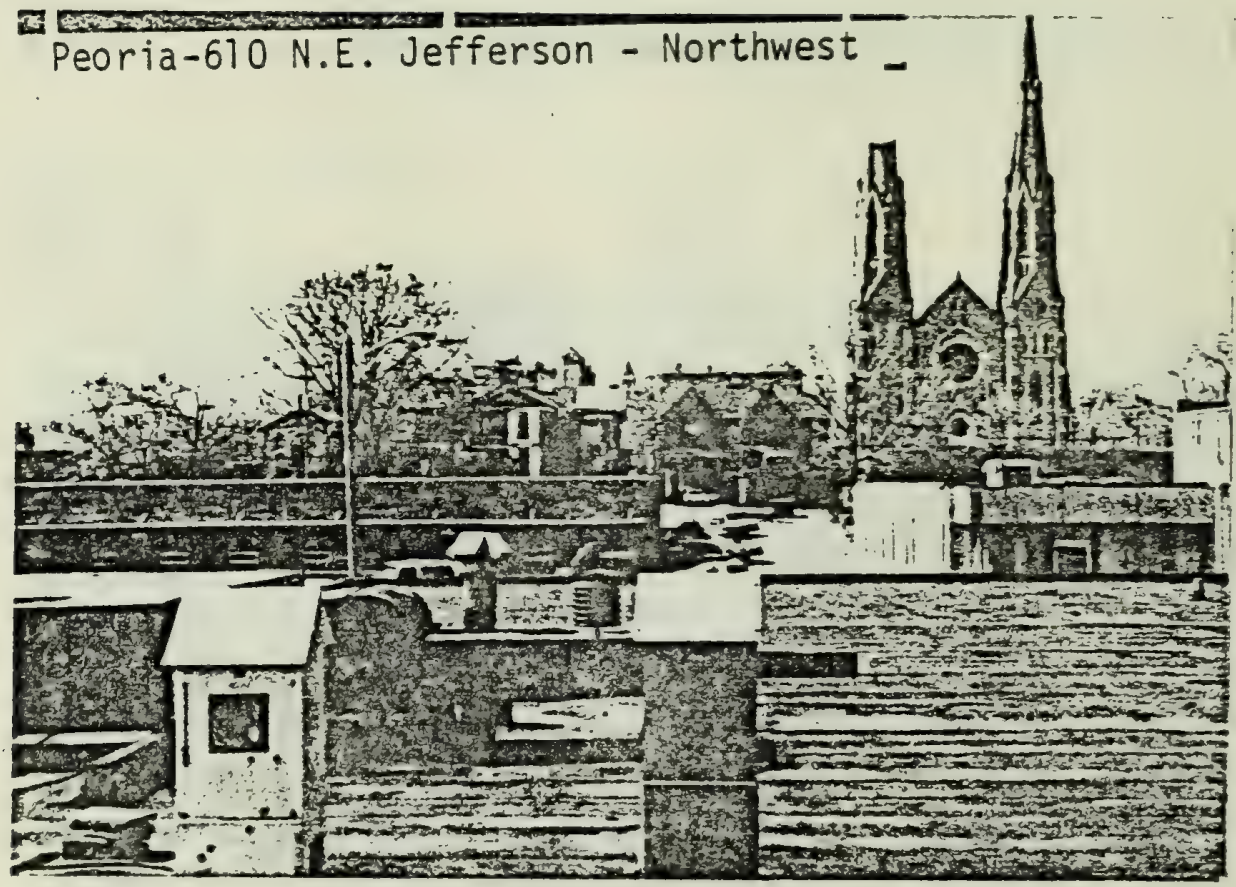

Peoria - North West 


\begin{tabular}{|c|c|}
\hline TER NIIMEER : 1279197 & $\begin{array}{l}\text { NETWIIFK : } \\
\text { SITE : }\end{array}$ \\
\hline CTION DATE & OEJECTIVE MAG. : 10 \\
\hline
\end{tabular}

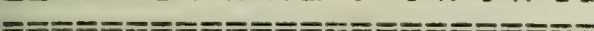

MINEFIALS

QUIAFTZ/FELISEAFE

LIMESTONE

IRON OXIIIES

OTHER MINERALS
17. 4

16. 9

22. 7

23. 9 b. $4-36.2$

4. $5-36.2$

12. $3-36.2$

6. 4-51. 1

7

0

40
37
5
0

27

40

1

0

SUOT

GLASSY FLYASH SFHEFES

BIOLOGICAI_

FOLLEN \& SFIRE:

FLANT TISEIIE

STAFI:H

26. 4

11. 4

1巨. 7

21.9
18. $1-36.2$

‥ $4-19.1$

12. $8-25.6$
18. $1-25.6$
13

3

15

2

$<1$

FUEEER:

\section{FAFTILLE SIZE IIISTFIEIITION}

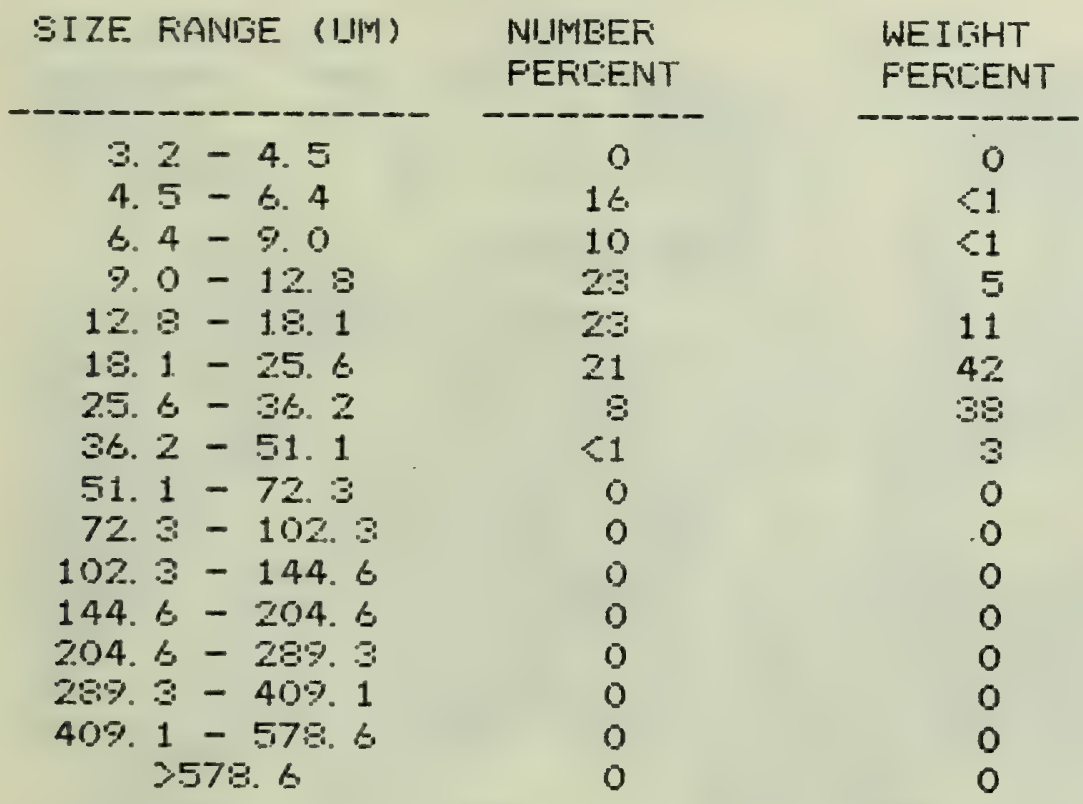


Total Suspended Particulate $\mu g / \mathrm{m}^{3}$

$\begin{array}{cclr}\text { Particulate: Minerals } 143.5 & \text { Combustion } & 12.3 \\ \text { Biological } 14 & \text { Other } & 3.5 \\ \text { Low temperature ashing, \% loss } & & 2.2\end{array}$
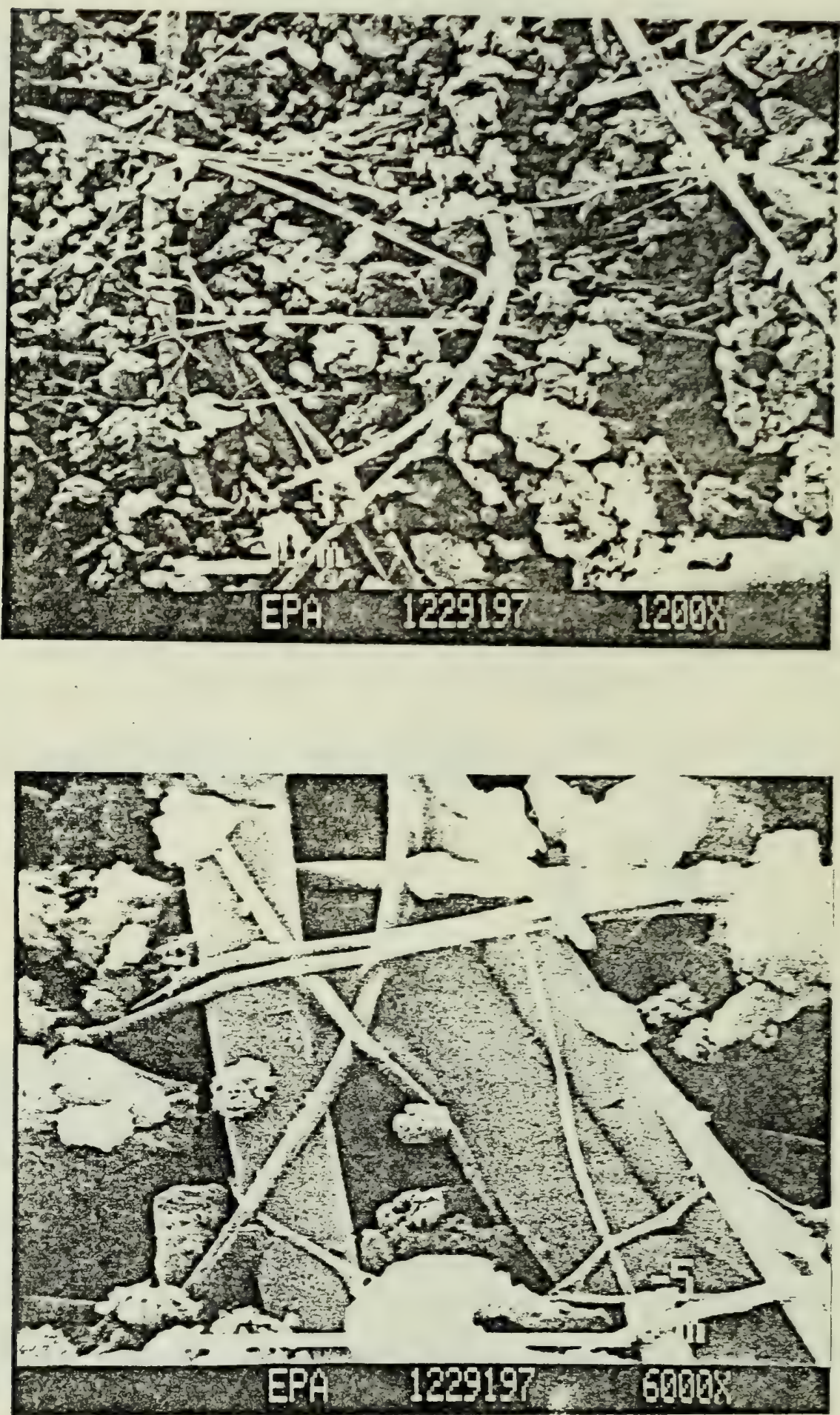


\begin{tabular}{|c|c|c|c|c|}
\hline $\begin{array}{l}\text { TER NUMBER : } 1229193 \\
\text { LECTION DATE : } 04 / 04 / 81 \\
===================\end{array}$ & $\begin{array}{l}\text { NETWIOFIK } \\
\text { SITE : } \\
\text { QEUJECTIV } \\
=========\end{array}$ & $\begin{array}{l}\text { FEORIJA } \\
\text { AB: }: 10 \\
=========\end{array}$ & $===x====$ & \\
\hline COMPONENT & $\begin{array}{l}\text { AVERAIIE } \\
\text { PART. } \subseteq \text { IZE }\end{array}$ & RANGE & $\begin{array}{l}\text { WEI IHT } \\
\text { FER'EENT }\end{array}$ & $\begin{array}{l}\text { Number } \\
\text { Percent }\end{array}$ \\
\hline \multicolumn{5}{|l|}{ NERALS } \\
\hline LIMESTONE & $\begin{array}{l}24.6 \\
14.7\end{array}$ & $\begin{array}{l}9.0-51 \cdot 1 \\
\text { 3. } 2-36 \cdot 2\end{array}$ & $\begin{array}{l}49 \\
17\end{array}$ & $\begin{array}{l}17 \\
32\end{array}$ \\
\hline $\begin{array}{l}\text { IRON OXIDES } \\
\text { OTHER MINEFALS }\end{array}$ & 28.0 & 12. $8-51.1$ & 16 & 4 \\
\hline \multicolumn{5}{|l|}{ IMEUSTION FRONUICTE } \\
\hline GLASSY FL.YASH SFHERES & 27.5 & 6. $4-72.3$ & $\begin{array}{r}10 \\
0\end{array}$ & $\begin{array}{c}18 \\
0\end{array}$ \\
\hline \multicolumn{5}{|l|}{ 3IOLOGICAL. } \\
\hline $\begin{array}{l}\text { FULILEN \& SFUKEES } \\
\text { FLANT TISOIIE }\end{array}$ & 28.0 & 12. $8-51.1$ & 7 & 6 \\
\hline $\begin{array}{l}\text { SLANT TISSIIE } \\
\text { STARIOH }\end{array}$ & $\begin{array}{l}11.4 \\
13.2\end{array}$ & $\begin{array}{l}\text { 6. } 4-1 \Xi \cdot 1 \\
\text { 9. } 0-19.1\end{array}$ & $\begin{array}{l}<1 \\
<1\end{array}$ & $\begin{array}{r}22 \\
1\end{array}$ \\
\hline $\begin{array}{l}\text { 1ISCELLAANEDUIS } \\
\text { FERROUS METAL } \\
\text { FUUEEER }\end{array}$ & & & $\begin{array}{l}0 \\
0\end{array}$ & $\begin{array}{l}0 \\
0\end{array}$ \\
\hline
\end{tabular}

F'ARTICLE SIZE DISTRIEUITION

SIZE RIANISE (UM) NUMEER

FEFICENT

$3.2-4.5$

4. $5-6.4$

6. $4-9.0$

9. $0-12.9$

12. $8-18.1$

18. 1 - 25. 6

25. $6-36.2$

36. $2-51.1$

$51.1-72.3$

72. $3-102.3$

102. $3-144.6$

144. $6-204.6$

204. $8-289.3$

$289.3-409.1$

$40 \% .1-578.6$ 2578. \& 
Inhalable Particulate

$$
\mu g / m^{3}
$$

Particulate: Minerals 95.1 Combustion 11.6 Biological 8.1 Other -

Low temperature ashing, \% loss

0.0
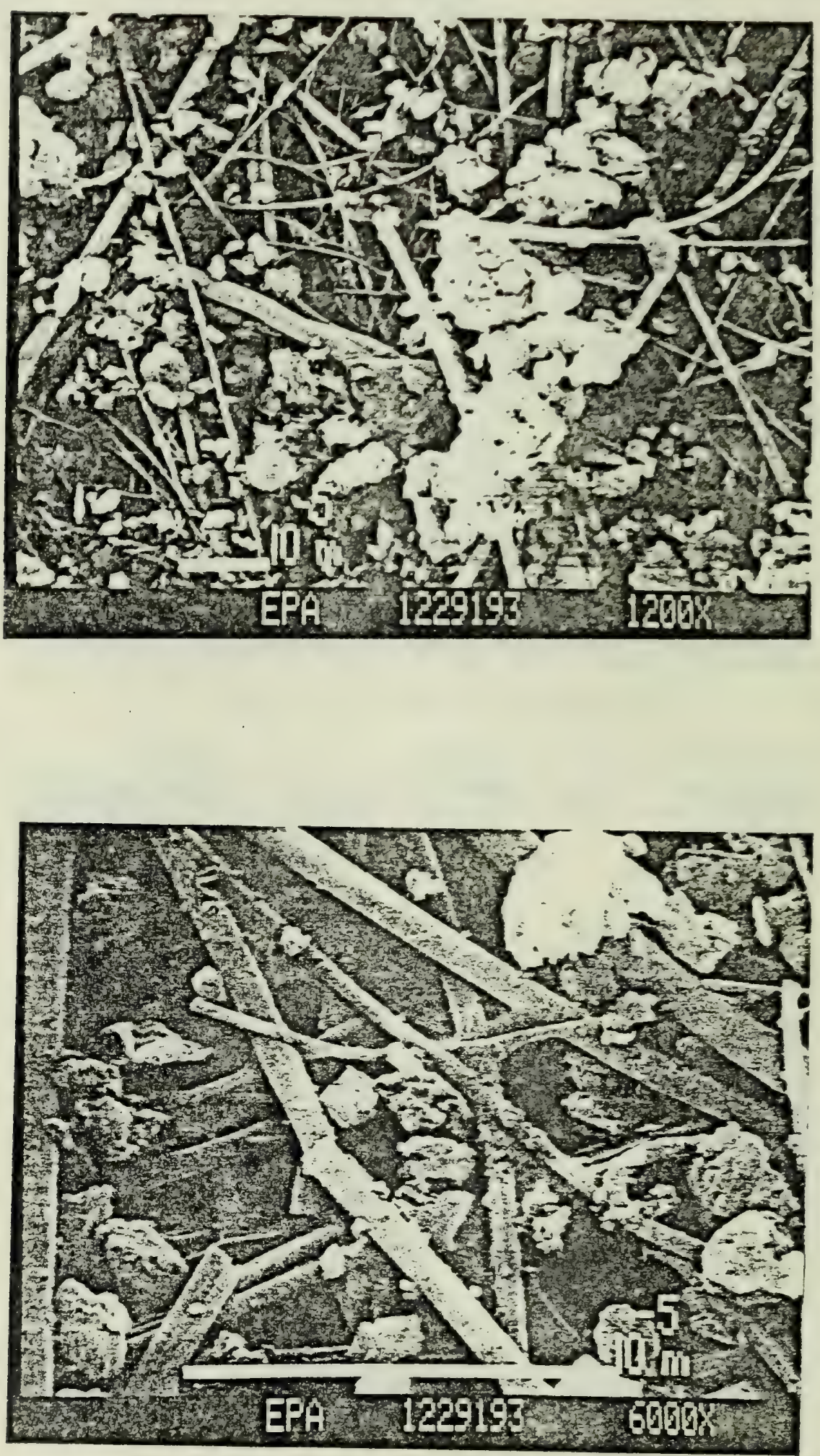
Total Suspended Particulate $\mu \mathrm{g} / \mathrm{m}^{3}$

$\begin{array}{cclc}\text { Particulate: Minerals } & 63.5 & \text { Combustion } & 4.4 \\ \text { Biological } & 19.1 & \text { Other } & - \\ \text { Low temperature ashing, \% loss } & & 59.2\end{array}$

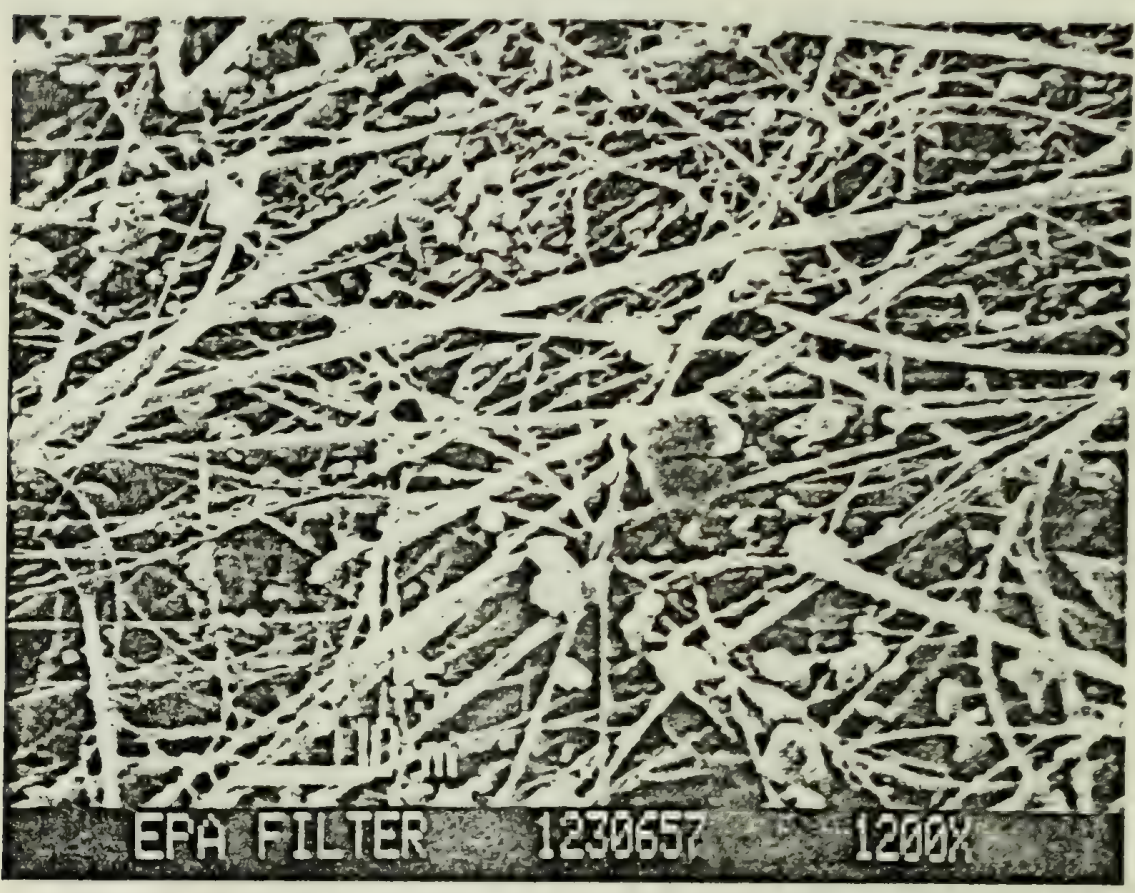

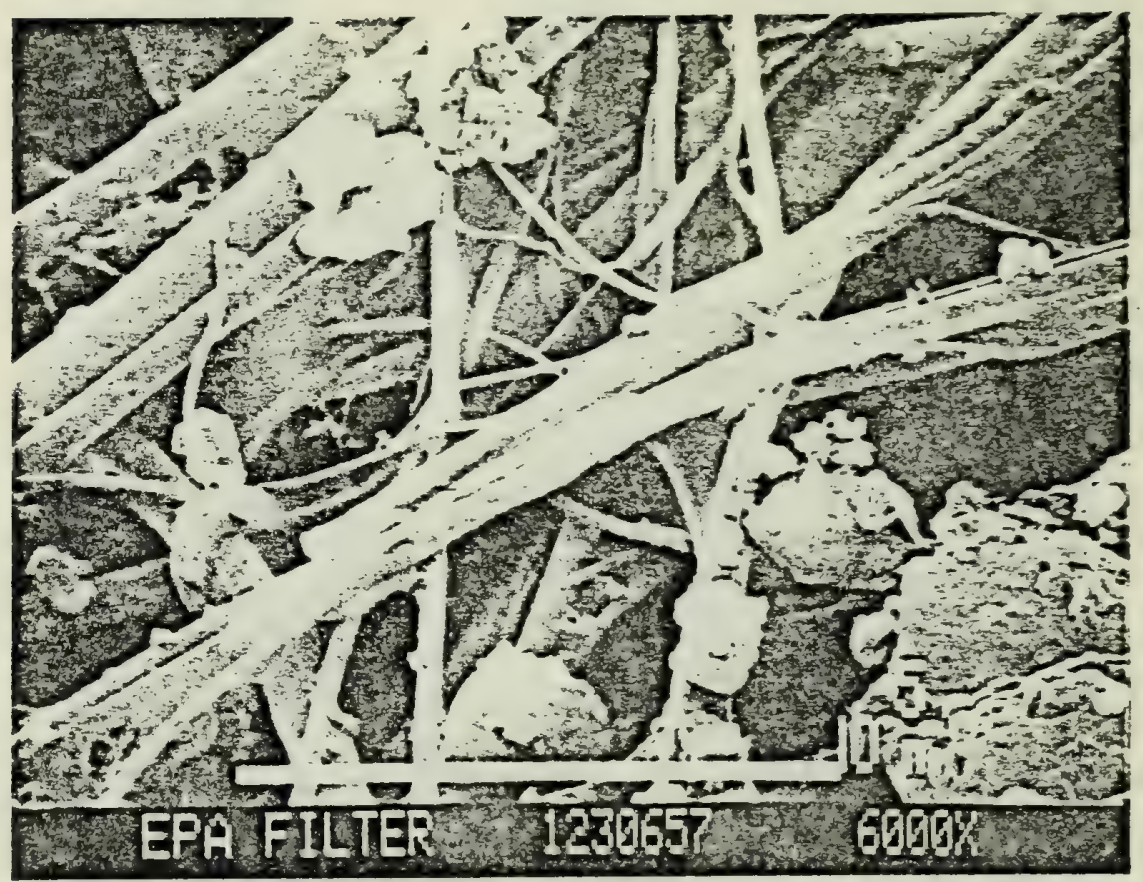


$===================$
[LTER NUMBER : 1230654

JLIIECTION DATE : 06/03/81

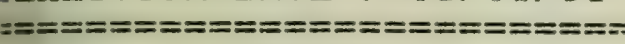
COMPINENT
NETWDRK :

SITE :

DE.IECTIVE MAT: 25

\section{PEOFIIA}

\begin{tabular}{|c|c|c|c|c|}
\hline COMFOINENT & $\begin{array}{l}\text { AVERAGE } \\
\text { FAFT. SIZE }\end{array}$ & FANIEE & $\begin{array}{l}\text { WE I BHT } \\
\text { PEFICENT }\end{array}$ & $\begin{array}{l}\text { Number } \\
\text { Percent }\end{array}$ \\
\hline \multicolumn{5}{|l|}{ MINERALS } \\
\hline QUAFTZ/FEI_DSFAFS: & 10.9 & 2. $5-28.8$ & 37 & 35 \\
\hline LIMESTONE & 7. 7 & 1. $8-20.4$ & 21 & 44 \\
\hline IRON OXINES & 9.8 & $3.6-20.4$ & 3 & 2 \\
\hline OTHER MINEFALS & & & 0 & 0 \\
\hline \multicolumn{5}{|l|}{ COMEUSTION FFIILUIITS } \\
\hline SODT & 10. 9 & 2. $5-28.8$ & 17 & 18 \\
\hline GLASSY FLYASH SFHERES & & & 0 & 0 \\
\hline \multicolumn{5}{|l|}{ BIOL_DGICAL } \\
\hline POLLEN \& SFORES & 24. 3 & 5. $1-81.5$ & 20 & 2 \\
\hline FLANT TISEIIE & 18. 1 & 10. $2-29.8$ & 2 & 1 \\
\hline STARIL.H & 13. 1 & 7. $z-20.4$ & $<1$ & 1 \\
\hline \multicolumn{5}{|l|}{ MISI:EL_LANEOUIS } \\
\hline FEFRIUIS METAL & . & & 0 & 0 \\
\hline RUEE:EK & & & 0 & 0 \\
\hline
\end{tabular}

FARTICLE SIZE DISTRIEUTIUN

SIZE FIANTEE (IIM)

NIIMEEEF

FEFICENT

1. $3-1.8$

1. $8-2.5$

2. $5-3.6$

3. $6-5.1$

5. $1-7.2$

$7.2-10.2$

10. $2-14.4$

14. $4-20.4$

20. $4-28.8$

20. $5-40.7$

$40.7-57.6$

57. 6. -81.5

31. $5-115.2$

115. $2-162.9$

$1 \leqslant 2.9-230.4$

$32: 30.4$
WE I BHT

FEFICEIVT

0
2
17
11
25
19
20
3
3
0
0
1
0
0
0
0

0
$<1$
$<1$
$<1$
5
9
26
13
26
0
0
19
0
0
0
0


Inhalable Particulate

$\mu g / \mathrm{m}^{3}$

$\begin{array}{cccc}\text { Particulate: } \begin{array}{c}\text { Minerals } \\ \text { Biological } 13\end{array} \text { Other } & \text { Combustion } & 10 \\ \text { Low temperature ashing, } \% \text { loss } & & 24.1\end{array}$
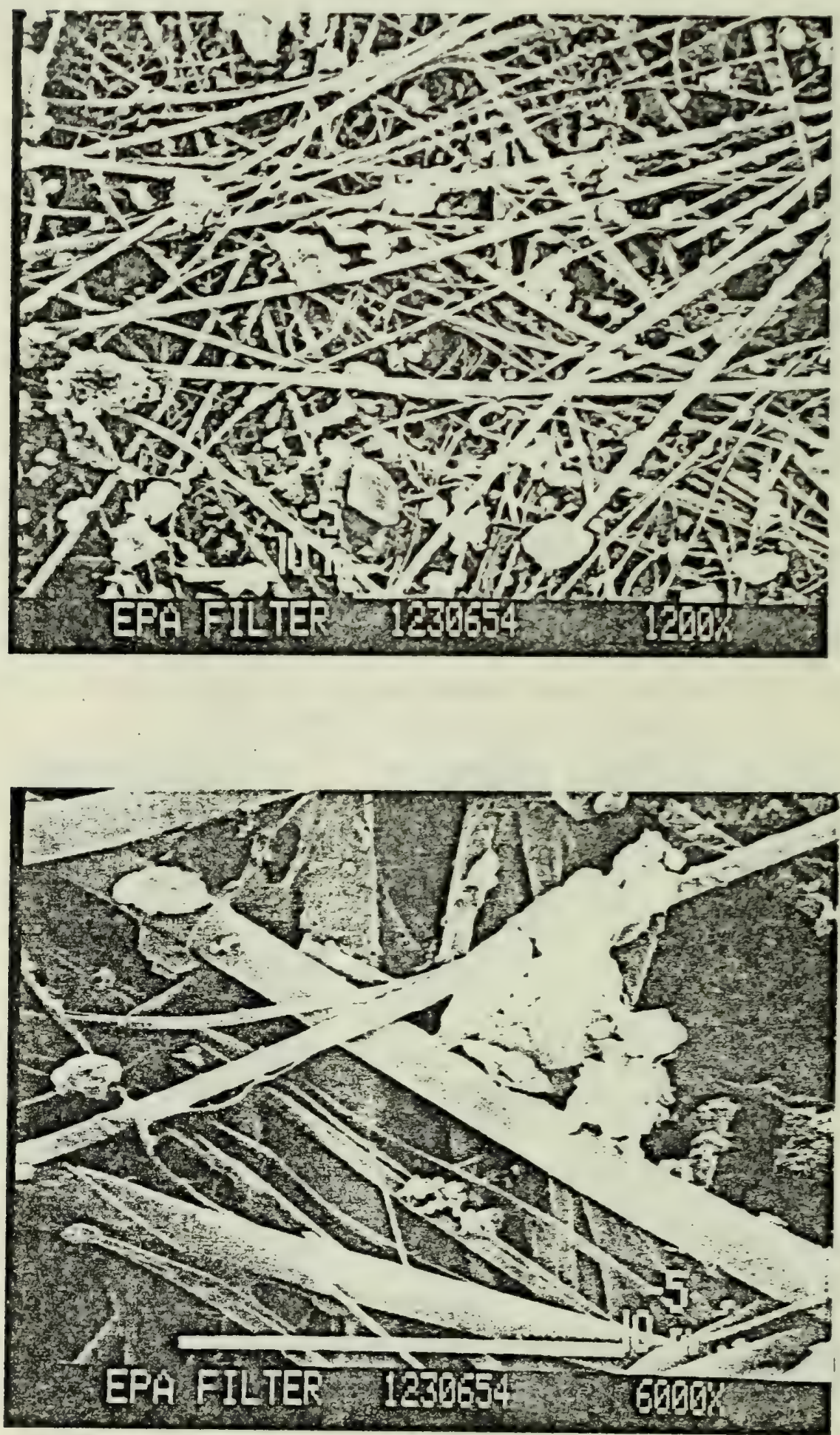


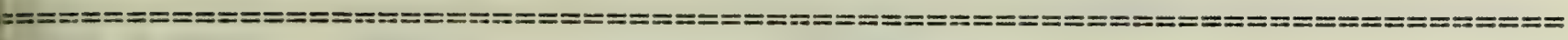

[L.TER NIIME:EF : 1282108

NETWIAKK :

SITE :

FEORIA

ILLECTIIN IIATE. : $12 / 18 / 81$

QE.JEITIVE MAL. : 25

AVERAGIE

PART. SIZE.
FIANIEE

8. 7

12. 4

6. 4

GI_ASSY FL_YASH SFHEFES

QI_IARTZ.FELDSFAF:S

LIMESTINE

OTHER MJNEFALS

COMEILSTION FRIOLIIIETS
6. 9
SODT

2. $5-20.4$

2. $5-40.7$

3. $6-10.2$

2. 5-14. 4

DGICAL

FILLLEN \& SFIIFE:

FLANT TIS:BUE

STAFLH

10. 1
11. 1
10. 5

3. $5-20.4$

WE I BHT Number

FERLEINT Percent

\section{MISTELLANETUIS}

FERFIDI:S: METAL.

RIIEEEF'

5. $1-20.4$

7. $2-14.4$

$\begin{array}{rr}8 & 8 \\ 83 & 49 \\ <1 & 1 \\ 0 & 0\end{array}$

37

00

FAFTICILE SIZE DISTRIEIITION

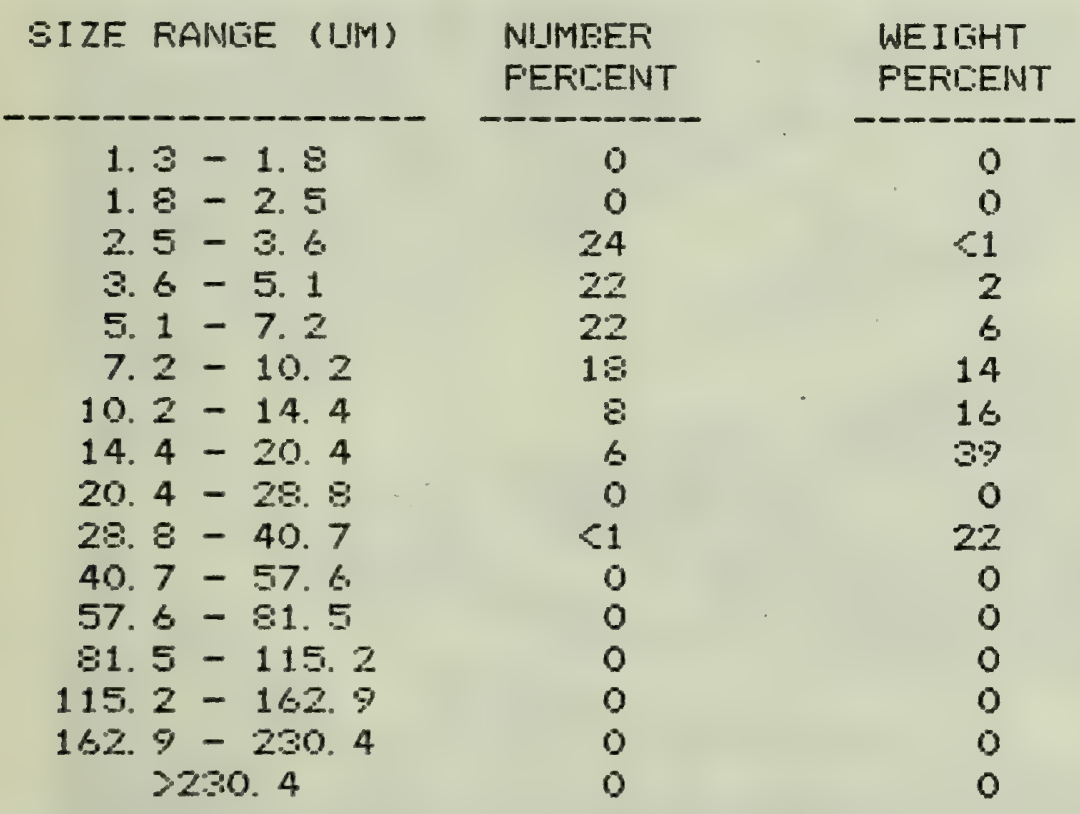


Total Suspended Particulate $\mu \mathrm{g} / \mathrm{m}^{3}$

$\begin{array}{cclc}\text { Particulate: Minerals } & 45.5 & \text { Combustion } & 2.5 \\ \text { Biological } 15 & \text { Other } & - \\ \text { Low temperature ashing, } \% \text { loss } & & 33.9\end{array}$
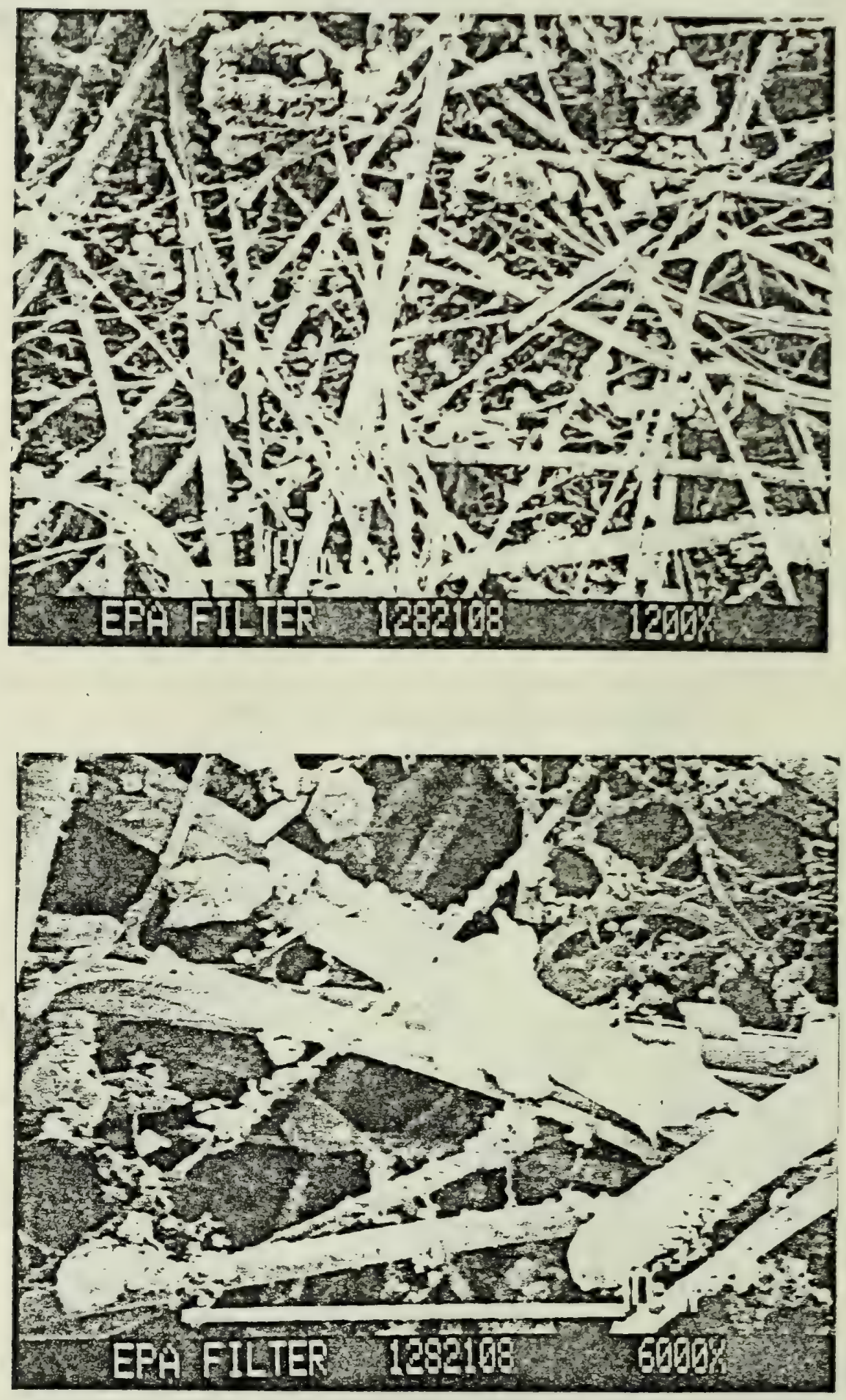


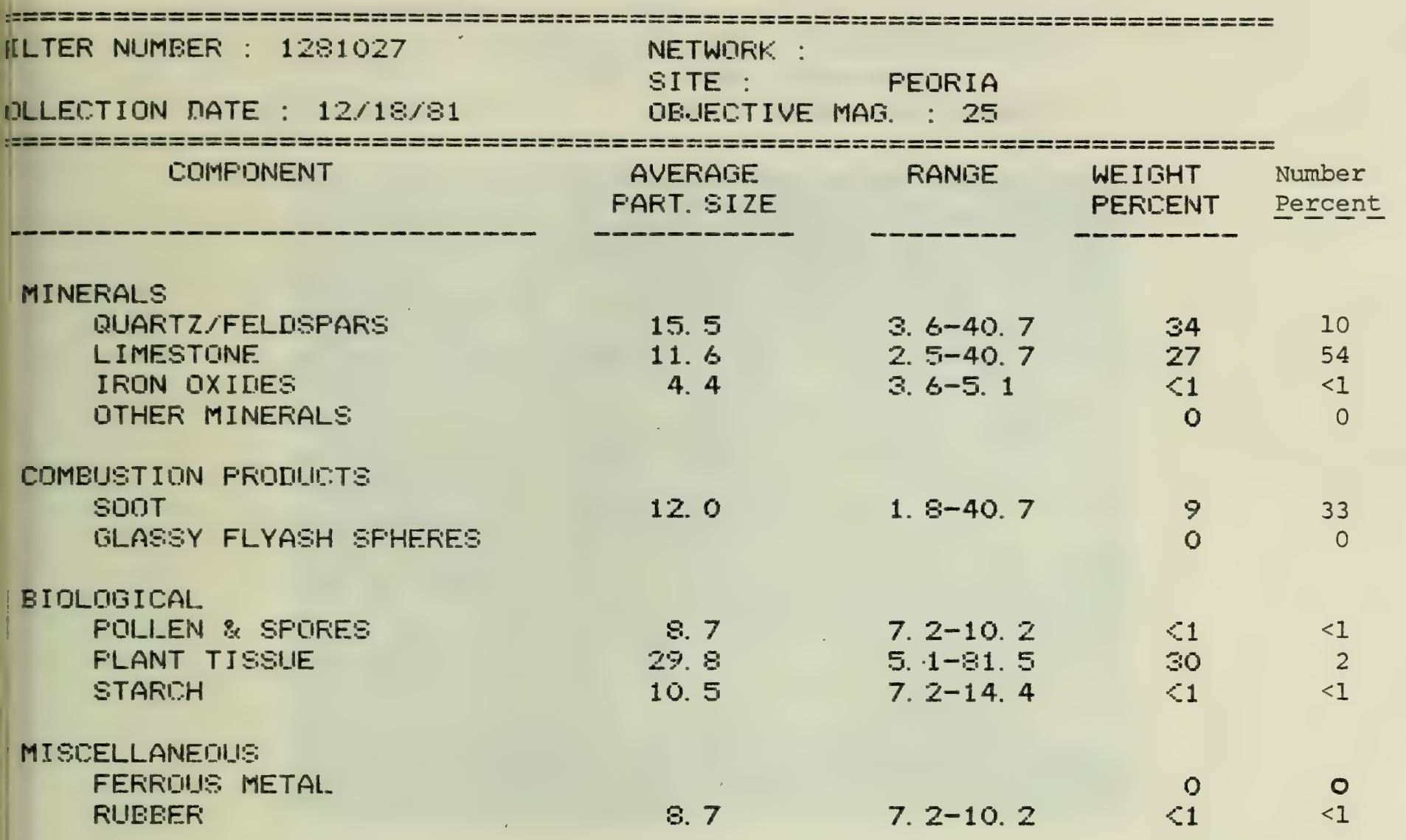

FAFTIOLE SIZE DISTRIEIUTION

\begin{tabular}{|c|c|c|c|}
\hline SIZE & RANIEE (IIM) & $\begin{array}{l}\text { NUIMEER } \\
\text { FERLEENT }\end{array}$ & $\begin{array}{l}\text { WEIGHT } \\
\text { F'ERICENT }\end{array}$ \\
\hline & $3-1.8$ & 0 & 0 \\
\hline & $8-2.5$ & 10 & $<1$ \\
\hline 2. & $5-36$ & 31 & $<1$ \\
\hline & $6-5.1$ & 19 & 1 \\
\hline & $1-7.2$ & 16 & 3 \\
\hline & $2-10.2$ & 17 & 8 \\
\hline 10. & $2-14.4$ & 7 & 10 \\
\hline 14. & $4-20.4$ & $<1$ & 2 \\
\hline 20. & $4-28.6$ & $<1$ & 8 \\
\hline 28. & $8-40.7$ & 1 & 40 \\
\hline 40. & $7-57.6$ & 0 & 0 \\
\hline 57. & $s-81.5$ & $<1$ & $2 \theta$ \\
\hline 81 & $5-115.2$ & 0 & 0 \\
\hline 115 & $2-1627$ & 0 & 0 \\
\hline $1 \in: 2$. & $9-200.4$ & 0 & 0 \\
\hline & 3230.4 & 0 & 0 \\
\hline
\end{tabular}


Inhalable Particulate $\quad \mu g / \mathrm{m}^{3} \quad 41$

Particulate: Minerals 25 Combustion 3.7

Biological 12.3 Other -

Low temperature ashing, \% loss 3.3
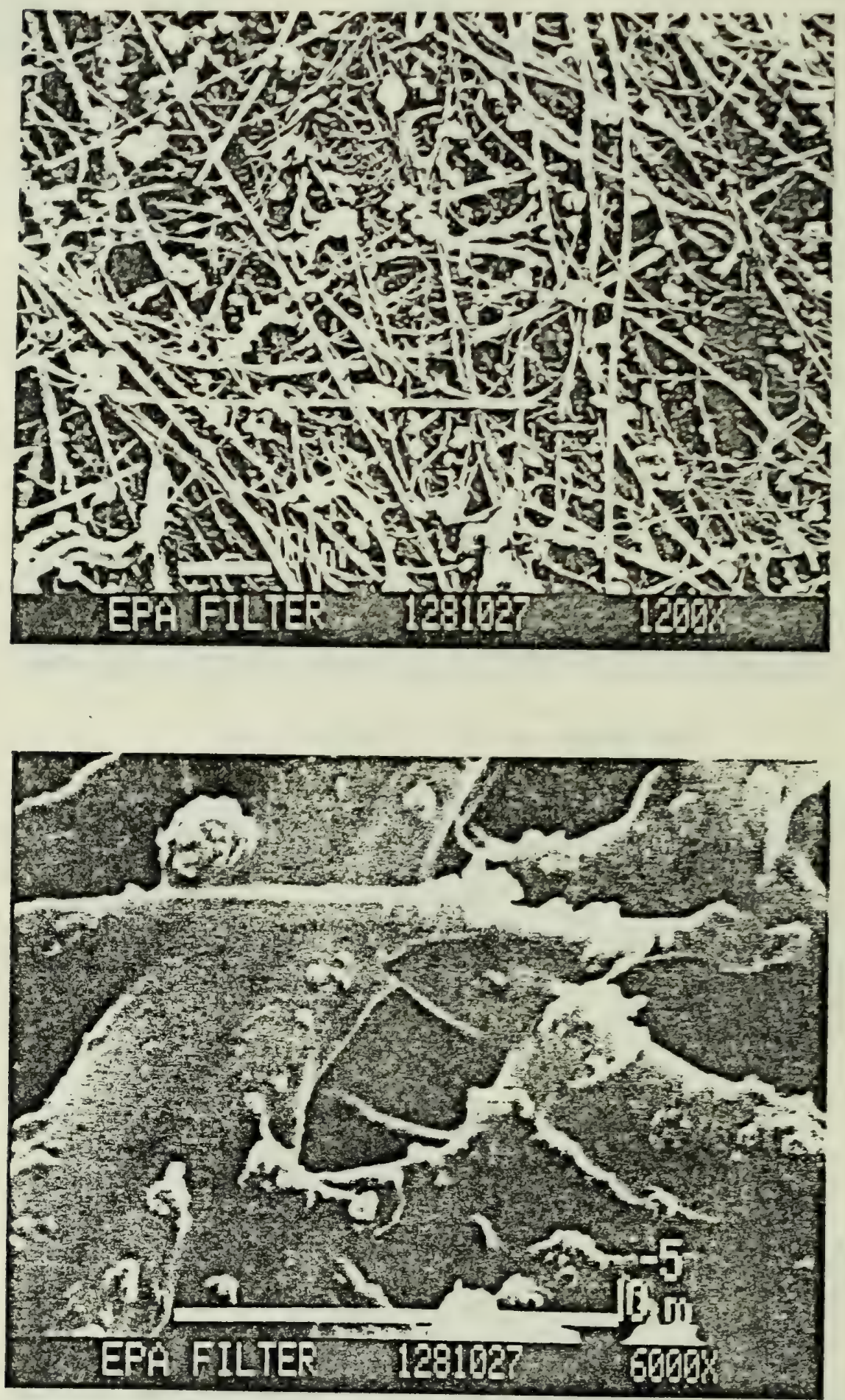


\section{A. Site Identification}

1. City: Granite City

2. Site Name and Address: Fire Station $\|_{1}, 23 r d \&$ Madison

3. County: Madison Township: Granite City

4. USGS Topographical Map Name and Scale: Granite City, II-MO $1: 24000$

5. Site Elevation (Feet): 420

B. Site Classification/Representativeness

1. Dominating Influence on Site: Industrial, Commercial

C. Source Impact

1. Stationary Sources that may Influence Site:

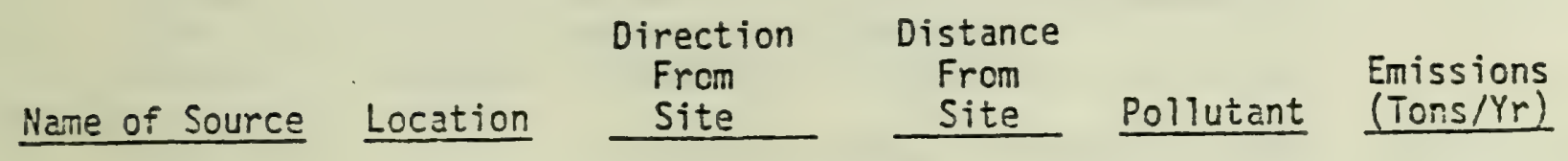

American Steel Foundaries 1700 Walnut W $1.0 \mathrm{mi}$ TSP 11,588 U.S Cntr. Ni haus

Nestle Co. 2101 Adams WSW

$1.7 \mathrm{mi}$ S02 iedring-

-

WNW

$0.6 \mathrm{mi}$

TSP

$\mathrm{SO} 2$

1,232 324

\section{Re}

Ame 
Granite City Steel

(BOF)

(Sinter Plant)

(Coke Plant) 20th \& State

(20th \& SSW-SW

Madison)

(20th \& SSE

Edwards-

ville)

(Nameoki \&

Edwards -

ville)

2801 Rock Rd.

ESE

$1.0 \mathrm{mi}$

$\mathrm{CO}$

$\mathrm{HC}$

$0.9 \mathrm{mi}$
159,943

9,201

2,843

Tri-State Regional Port District

International Mill

Service

22nd \&

Edwards -

SE

NOX

92,283

1,284

ville Rd.

2. Mobile Sources that may Influence the Site:

Names of Roadways:

Madison $\quad$ 23rd

Iowa

Type:

Arterial Street Local Street Local

Distance of Roadway

from Site ( $\mathrm{ft}$ )

140

155

120

Composition of Roadway

Asphalt

Asphalt

Asphalt

Number of Traffic Lanes

4

2

1

Average Daily Traffic

19,300

6000

1000

Average Vehicle Speed (mph)

25

20

20

One or Two Way Traffic

Two

Two

One (NE)

Number of Parking Lanes

None

None

Two

Are Parking Lanes Used

For Traffic Part of Day?

N/A

N/A

Yes

Is Dust Visibiity Retained

No

No

No

Does Roadway Have Curb

Yes

Yes

Yes

3. Area Sources that may Influence the Site:

Type of Source

St. Louis Met area
Direction

From Site

SSW
Distance

From Site

\section{Pollutant}

Part. as well as $\mathrm{SO}_{2}, \mathrm{HC}, \mathrm{NO}_{\mathrm{x}}$, and $\mathrm{O}_{3}$ 
D. Topography/Obstructions

1. General Characteristics Over a 2 Mile Radius From the Site: Smooth

2. Topographic Features that Influence the Site: (Types - hills, valleys, depressions, bodies of water, ridges, cliffs)

$\begin{array}{llll}\text { Type } & \text { Size } & \text { Direction } & \text { Distance } \\ & & \text { From Site } & \text { From Site }\end{array}$

None

3. Obstructions to Wind Flow

(Types - buildings, trees, ridges, cliffs)

Type

\begin{tabular}{ll} 
Size & Direction \\
\hline
\end{tabular}

Distance

From Site

None

E. Distance to the nearest National Weather Service (NWS) Site. Here are the distances and directions to the nearest NWS observing site from the inhalabie particulate monitor in Granite City. Distances are in statute miles; directions are degrees from true north.

Site Ad ress

Fire Station \#1

23rd and Madison
Nearest NWS Site

Lambert Airport/

St. Louis (STL)
Directions and Distance to NWS

$280^{\circ}$ at $13 \mathrm{mi}$. (W)

Bi-State Airport (CPS) is about 8 miles south of Granite City (to CPS from FS\#1, $190^{\circ}$ at $8.9 \mathrm{mi}$; from E $20 \mathrm{th}, 190^{\circ}$ at $8.3 \mathrm{mi}$. ).

Alton Airport (ALN) is about 14 miles north of Granite City (to ALN from FS\#T, $020^{\circ}$ at $14 \mathrm{mi}$; from $E 20 \mathrm{th}, 020^{\circ}$ at $14.8 \mathrm{mi}$ ).

Scott AFB (BLV) is about 19 miles southeast of Granite City (to BLV from FS\#1, $130^{\circ}$ at $19 \mathrm{mi}$; from E $20 \mathrm{th}, 125^{\circ}$ at $19 \mathrm{mi}$ ).

Data from all of these sites are available to the IEPA through WSI, but only ALN appears on our Service "A" teletype circuit. None of these sites is a NWS site, and no Local Climatological Data (LCDs) are issued for them. 


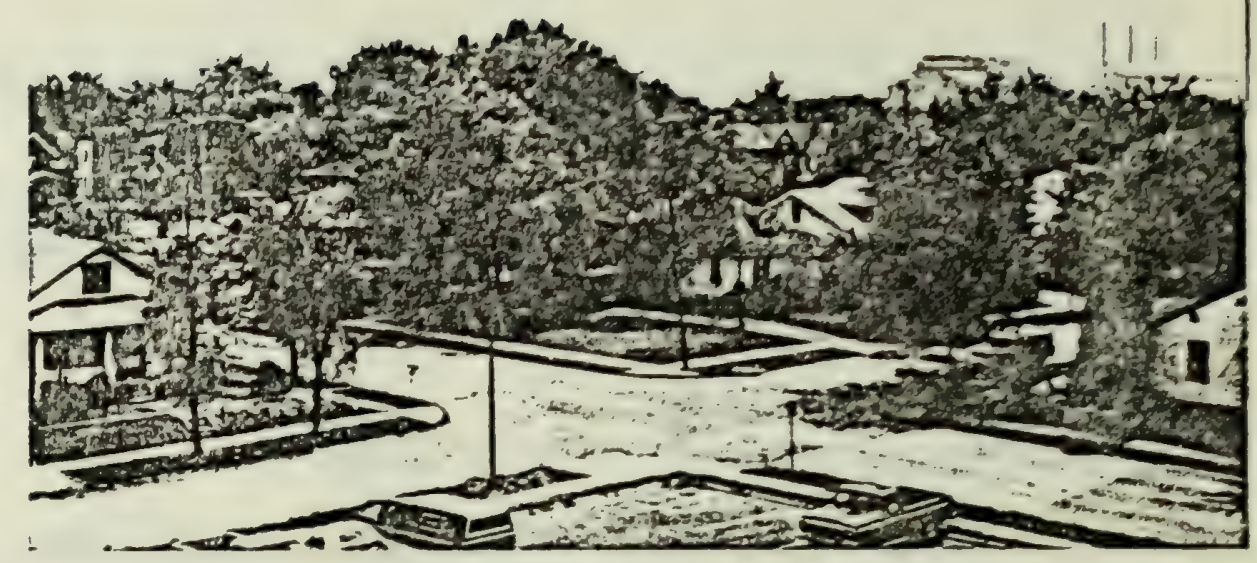

Granite City (Fire Station No. 1) - South

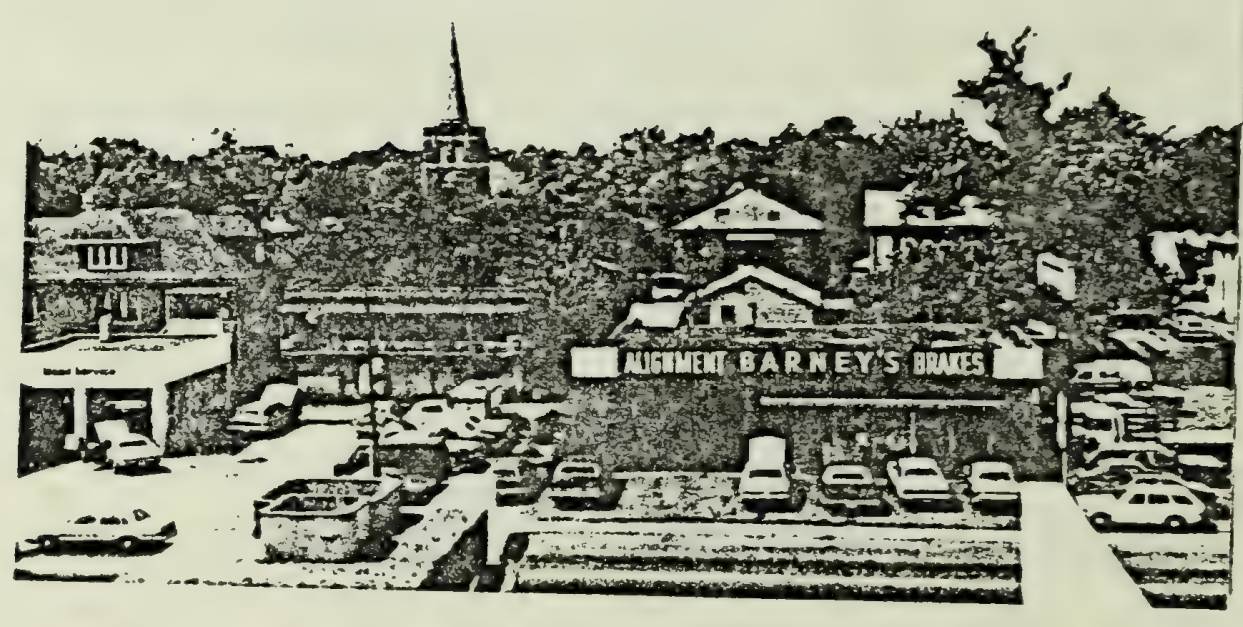

Granite City (Fire Station No. 1) North West 
Granite City Looking N

23rd \& Madison

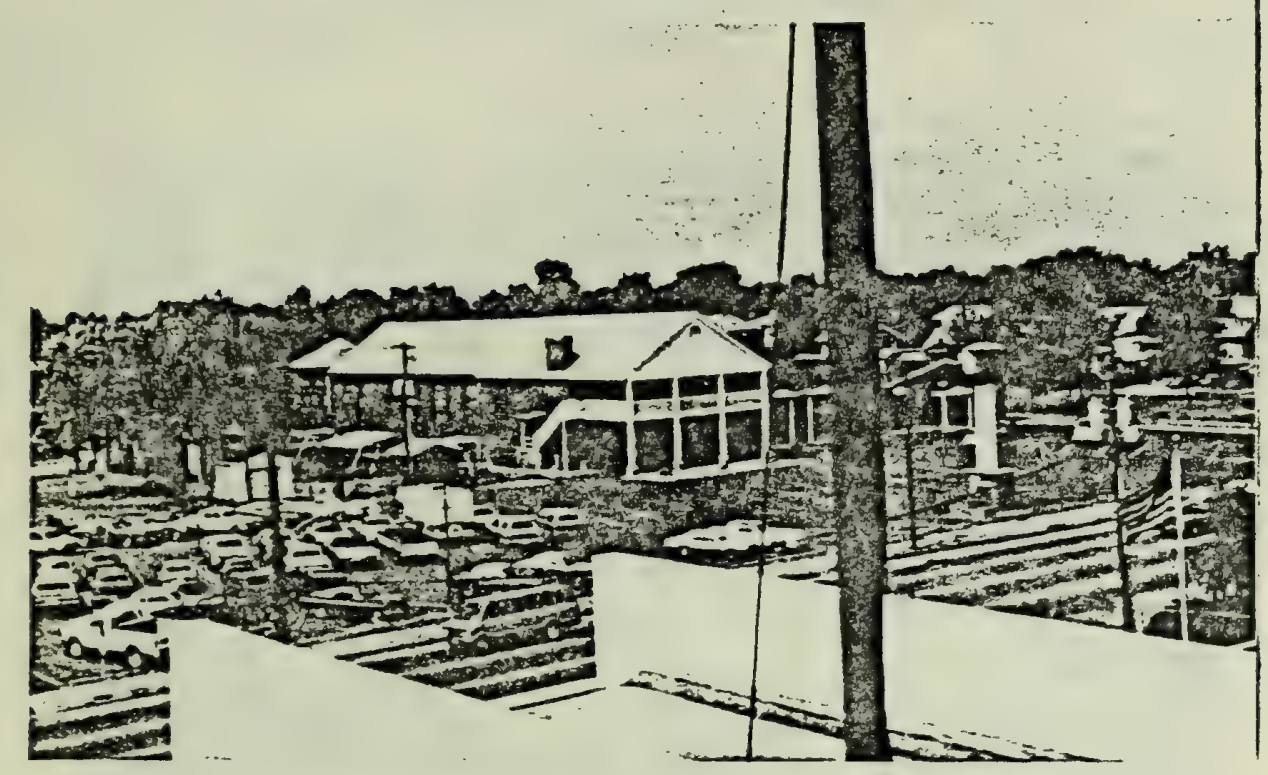

Granite City (Fire Station No. 1) North

Granite City

Looking $E$

23rd \& Madison

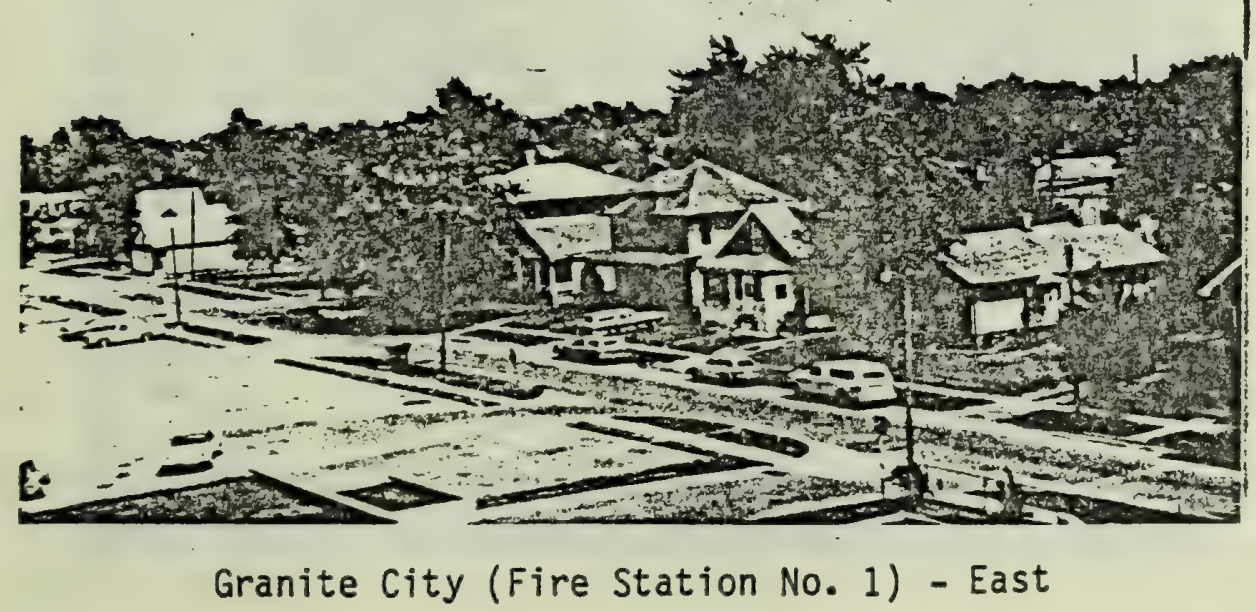


Total Suspended Particulate $\mu \mathrm{g} / \mathrm{m}^{3}$

$\begin{array}{rlr}\text { Particulate: Minerals } 158.2 & \text { Combustion } & 18.4 \\ \text { Biological } 5.5 & \text { Other } & 3.7 \\ \text { Low temperature ashing, \% loss } & & 11.3\end{array}$
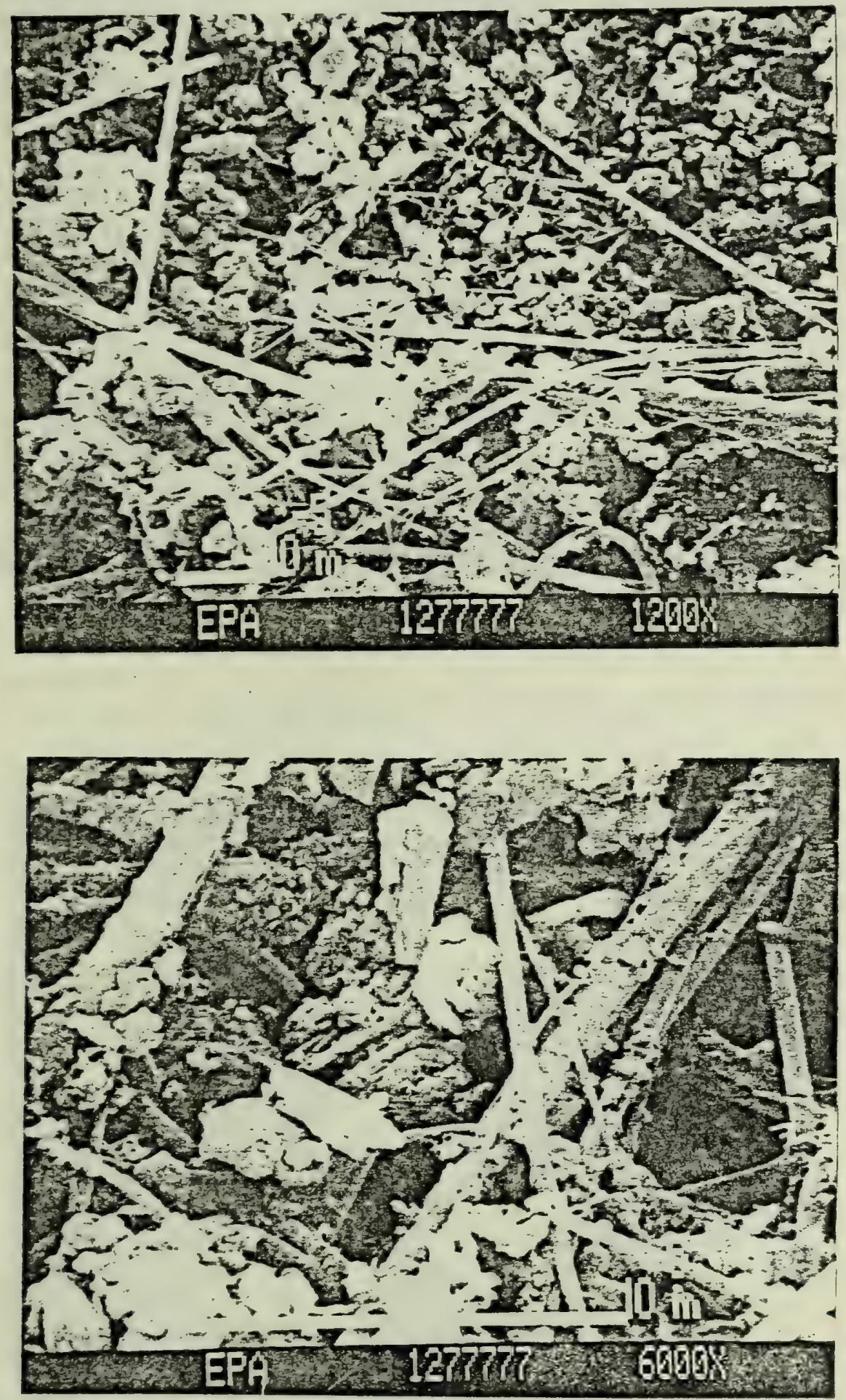


\begin{tabular}{|c|c|c|c|c|}
\hline \multirow{2}{*}{$\begin{array}{l}\text { FILTER NIMEEF : } 1277776 \\
\text { EIULLETIIN DATE : } 0: 114: 91 \\
========================\end{array}$} & NETWIIFI: & \multicolumn{2}{|c|}{ DSFANITEIITY/FIFEST. } & \multirow{2}{*}{1} \\
\hline & \multicolumn{3}{|c|}{ 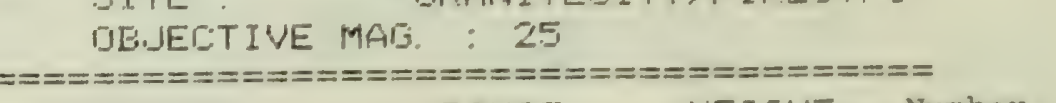 } & \\
\hline DDMFINENT & $\begin{array}{l}\text { AVEFIAISE } \\
\text { FAFIT. SIZE }\end{array}$ & RANIIE & $\begin{array}{l}\text { WEI IjHT } \\
\text { FERILENT }\end{array}$ & $\begin{array}{l}\text { Number } \\
\text { Percent }\end{array}$ \\
\hline \multicolumn{5}{|l|}{ MINEFIAL:S } \\
\hline $\begin{array}{l}\text { DIAFTZ.FELLISFAFE: } \\
\text { LIMESTINE }\end{array}$ & $\begin{array}{r}10.9 \\
7\end{array}$ & $\begin{array}{l}\text { 2. } 5-28.8 \\
1.8-20.4\end{array}$ & $\begin{array}{l}32 \\
26\end{array}$ & $\begin{array}{l}15 \\
42\end{array}$ \\
\hline IFION DXILES & 7.9 & 3. $6-14.4$ & 10 & 6 \\
\hline DTHEF MINEFIALS & & & 0 & 0 \\
\hline \multicolumn{5}{|l|}{ EIMELISTION FFIIUII:TS } \\
\hline GLASEY FLYASH SFHEFES & 7. & & 0 & 0 \\
\hline \multicolumn{5}{|l|}{ EIGLIIIICAL } \\
\hline $\begin{array}{l}\text { FILLFN } \% \text { SFIOFES } \\
\text { FLANT TISEILIE }\end{array}$ & $\begin{array}{r}7.1 \\
11.1\end{array}$ & $\begin{array}{l}=1-14.4 \\
5.1-20.4\end{array}$ & 10 & 4 \\
\hline ETAREH & & & 0 & 0 \\
\hline \multicolumn{5}{|l|}{ MISIELLANEDIS: } \\
\hline $\begin{array}{l}\text { FEFFIUIS: METAL } \\
\text { FIUEEEF }\end{array}$ & 7.2 & $2.5-14.4$ & 3 & $\begin{array}{l}3 \\
0\end{array}$ \\
\hline
\end{tabular}

FAFTILLE BIZE TISTFIEILITIOH

\begin{tabular}{|c|c|c|c|}
\hline EIZE $F$ & FIANIGE (UM) & $\begin{array}{l}\text { NUMEEER } \\
\text { FEFIEENT }\end{array}$ & $\begin{array}{l}\text { WEI IHHT } \\
\text { F'EFIL:ENT }\end{array}$ \\
\hline 1.: & $3-1.5$ & 0 & 0 \\
\hline 1. & $5-2.5$ & 1 & 1 \\
\hline 2.5 & $5-36$ & 18 & 1 \\
\hline 3. & $6-51$ & 26 & 4 \\
\hline 5. & $1-7.2$ & 29 & 17 \\
\hline 7. & $2-10.2$ & 14 & 20 \\
\hline 10. & $=-14.4$ & 10 & $\because 4$ \\
\hline 14. & $4-20.4$ & 3 & 21 \\
\hline 20 & $4-28=$ & $\therefore 1$ & 4 \\
\hline 28 & $B-40.7$ & 0 & 0 \\
\hline 40. & $7-57.6$ & 0 & 0 \\
\hline 57. & $\Leftrightarrow-91.5$ & 0 & 0 \\
\hline Eit. & $5-115.2$ & 0 & 0 \\
\hline 115 & $z-162.9$ & 0 & 0 \\
\hline 162 & -230.4 & 0 & 0 \\
\hline & 200.4 & 0 & 0 \\
\hline
\end{tabular}


Inhalable Particulate $\quad \mu \mathrm{g} / \mathrm{m}^{3}$

$\begin{array}{cclc}\text { Particulate: Minerals } 86.4 & \text { Combustion } & 29.7 \\ \text { Biological } 12.9 & \text { Other } & - \\ \text { Low temperature ashing, \% loss } & & 16.0\end{array}$
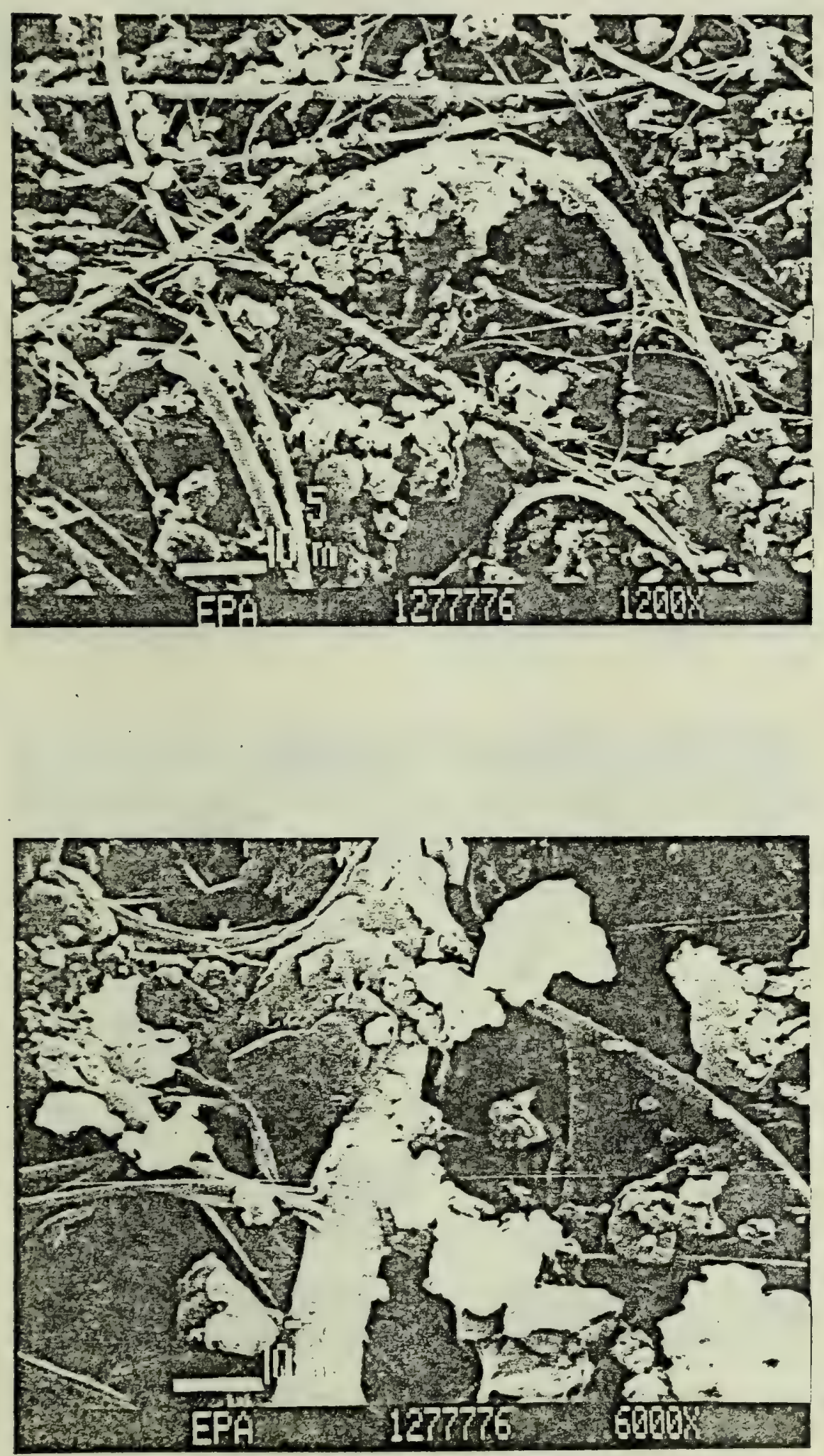
Inhalable Particulate $\quad \mu \mathrm{g} / \mathrm{m}^{3}$

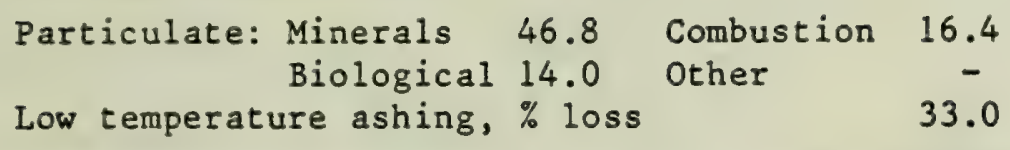
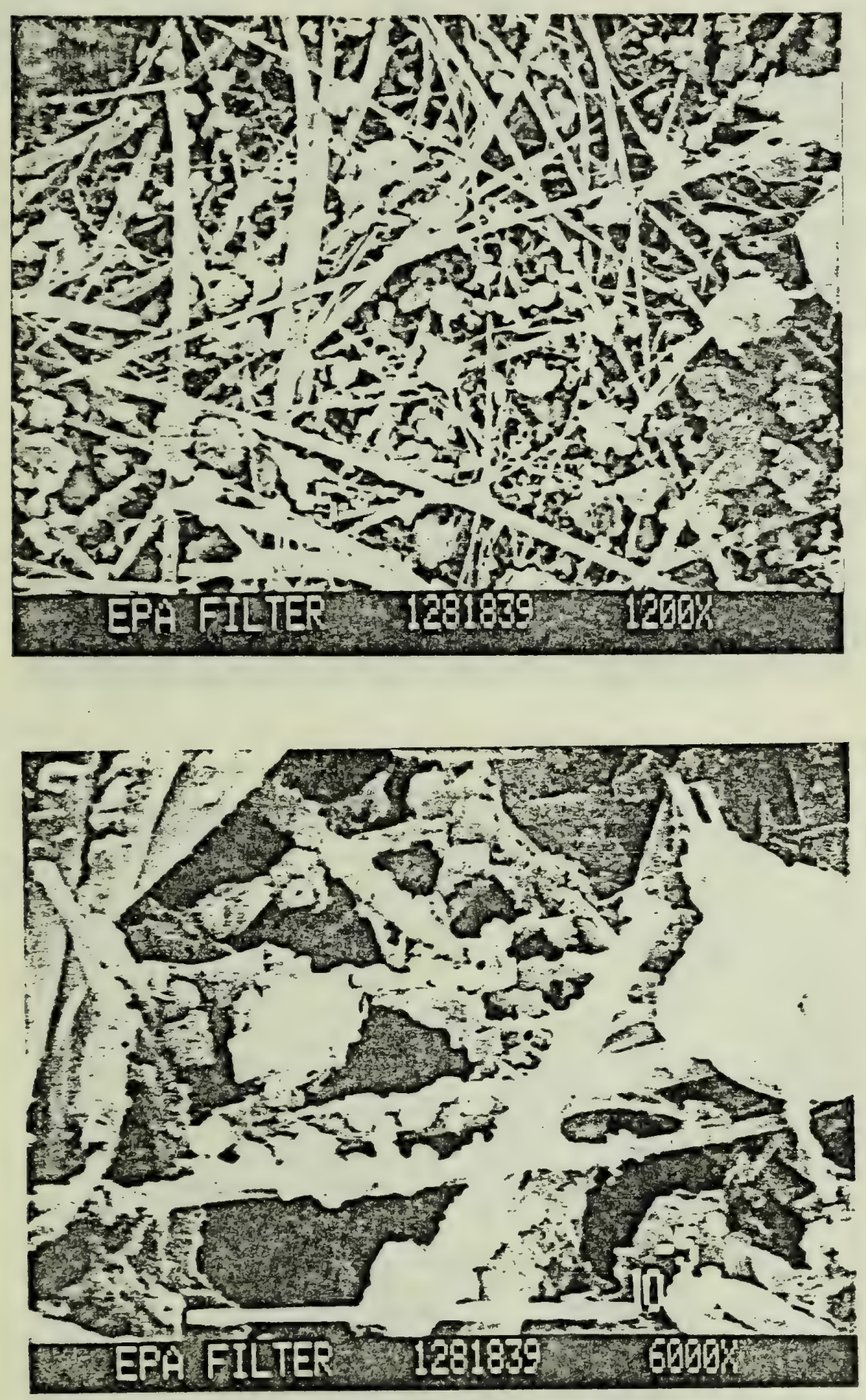
Total Suspended Particulate $\mu \mathrm{g} / \mathrm{m}^{3}$
Particulate: Minerals 35.9
Biological 20.7
Combustion
11.04
Other
2.1
Low temperature ashing, \% loss
33.2
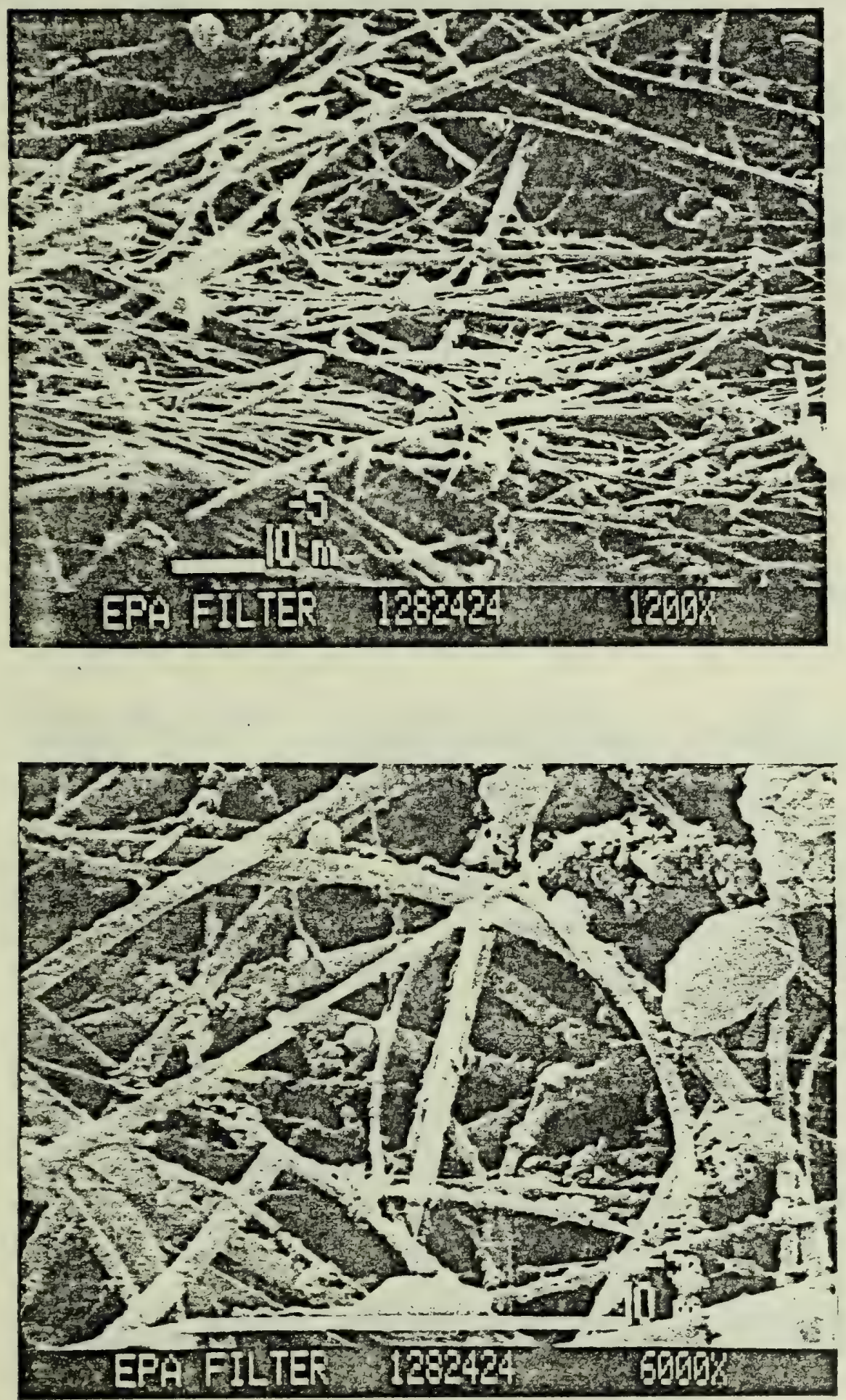


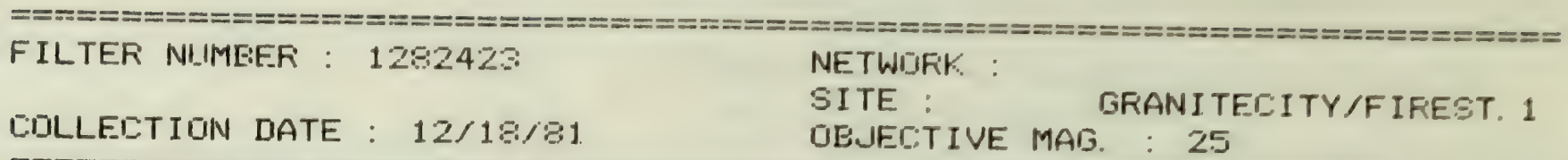

Number Percent

\section{MINEFIALS}

QUJAFITZ/FEI_DEPAFS

LIMESTINE

IFION OXIJES

UTHEF MINEFIALS
6.9

9. 5

7. 9

8. 7

CIMELSTION FROLUIIST
GILASSY FLYASH SFHEFES:

SIOTT

EICILOIII:AL.

FOLLEN \& SFOFIE:

FLANT TISEUIE

STAFICH
16. 3

$1 \% .6$

12. 3
2. $5-14.4$

1. $8-28,8$

3. $6-14.4$

2

8

1

0
5

22

3

0

43

0

2. $5-20.4$

10

8

7. $2-40.7$

7. $2-40.7$

10. $z-14.4$
$6 \%$

$<1$

MIST:ELLANEDIIS

FEFFIIIIS METAL

RUIEEEE:

SIIFT COMAL BIIFT CIIAL

\section{FARTICLE SIZE DISTRIEUTIIN}

\begin{tabular}{cc} 
SIZE FIANISE (UM) & NIIMEEF \\
FEFILENT \\
\hline $1.3-1.8$ & 0 \\
$1.8-2.5$ & 4 \\
$2.5-3.6$ & 23 \\
$3.6-5.1$ & 29 \\
$5.1-7.2$ & 10 \\
$7.2-10.2$ & 10 \\
$10.2-14.4$ & 8 \\
$14.4-20.4$ & 15 \\
$20.4-28.8$ & 7 \\
$28.5-40.7$ & 1 \\
$40.7-57.6$ & 0 \\
$57.6-81.5$ & 0 \\
$51.5-115.2$ & 0 \\
$115.2-162.9$ & 0 \\
$162.9-230.4$ & 0 \\
3230.4 & 0
\end{tabular}

\begin{tabular}{c} 
WEIBHT \\
FEFICENT \\
\hline 0 \\
$<1$ \\
1 \\
1 \\
1 \\
4 \\
7 \\
31 \\
43 \\
12 \\
0 \\
0 \\
0 \\
0 \\
0 \\
0
\end{tabular}




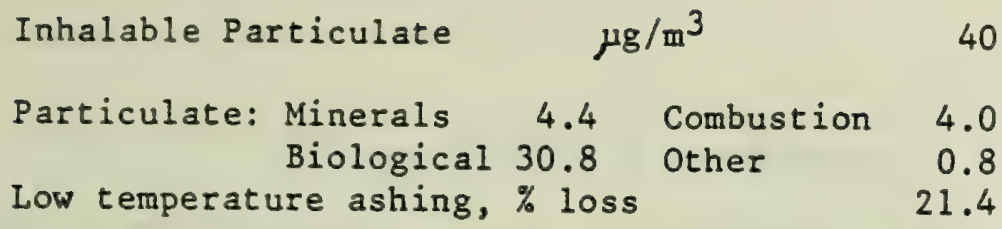
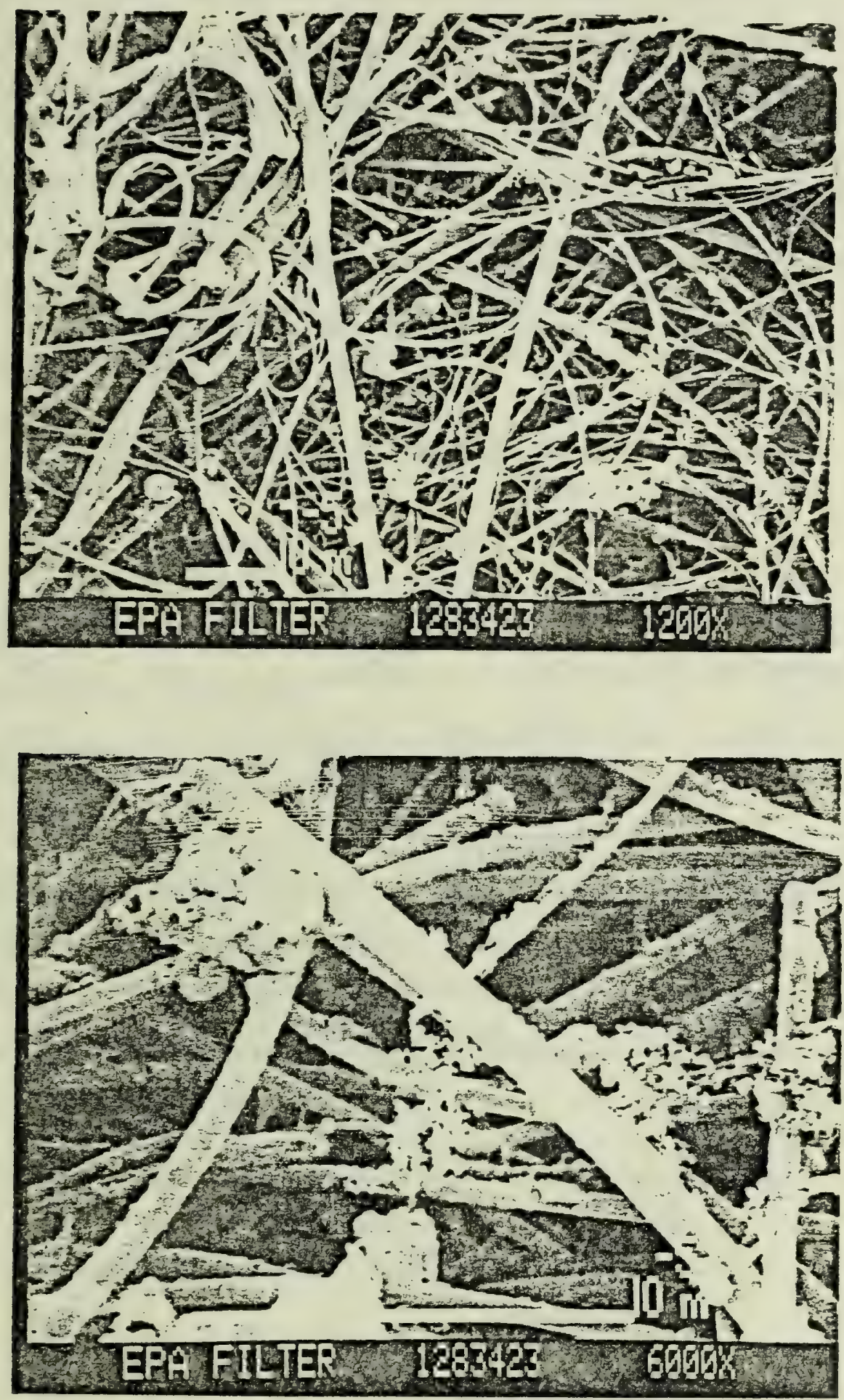
A. Site Identification

1. City: Granite City

2. Site Name and Address: Dallas Residence, 2001 E. 20th St..

3. County: Madisongo Township: Granite City 4. USGS Topographical Map Name and Scale: Granite City, I-MO,
$1: 24000$

5. Site Elevation (Feet): 415

B. Site Classification/Representativeness

1. Dominating Influence on Site: Industrial

C. Source Impact

1. Stationary Sources that may Influence Site

\begin{tabular}{|c|c|c|c|c|c|}
\hline Name of Source & $\begin{array}{l}\text { Directior } \\
\text { From } \\
\text { Site } \\
\end{array}$ & & $\begin{array}{l}\text { Distance } \\
\text { From } \\
\text { Site } \\
\end{array}$ & Pollutant & $\begin{array}{l}\text { Emissions } \\
\text { (Tons/Yr) } \\
\end{array}$ \\
\hline American Steel Foundaries & 1700 Walnut & WNW & $1.1 \mathrm{mi}$ & TSP & 9,697 \\
\hline U.S. Army Support Center & Niedringhaus Ave. & $W$ & $1.6 \mathrm{mi}$ & $\mathrm{SO}_{2}$ & 158 \\
\hline Nestle Co. & 2101 Adams & NW & $0.8 \mathrm{mi}$ & $\begin{array}{l}\text { TSP } \\
\text { SO2 } \\
\end{array}$ & $\begin{array}{r}2,553 \\
392 \\
\end{array}$ \\
\hline Reeves Concrete Prod. & 2801 Circle & $\mathrm{N}$ & $1.7 \mathrm{mi}$ & TSP & 14,723 \\
\hline Reilly Tar \& Chemical & $\begin{array}{l}\text { 19th \& Edwards- } \\
\text { ville }\end{array}$ & SSE & $0.8 \mathrm{mi}$ & TSP & 362 \\
\hline National Lead & 16th \& Cleveland & $w$ & $0.9 \mathrm{mi}$ & TSP & 2,783 \\
\hline Union Electric-Venice & Main St. & SW & $2.8 \mathrm{mi}$ & $\begin{array}{l}\mathrm{SO}_{2} \\
\mathrm{TSP}^{2} \\
\mathrm{SO}_{2} \\
\mathrm{NO} \\
\end{array}$ & $\begin{array}{r}624 \\
790 \\
6,649 \\
4,434 \\
\end{array}$ \\
\hline ADil-Granite City & 3601 Cargill & ENE & $3.5 \mathrm{mi}$ & $\begin{array}{l}\text { TSP } \\
\text { SO2 } \\
\end{array}$ & $\begin{array}{r}15,141 \\
204 \\
\end{array}$ \\
\hline $\begin{array}{l}\text { Granite City Steel } \\
\text { (BOF) } \\
\text { (Sinter Plant) }\end{array}$ & $\begin{array}{l}\text { 20th \& State } \\
\text { (20th \& Madison SW } \\
\text { (20th \& Edwards- } \\
\text { ville) }\end{array}$ & $\begin{array}{l}1-W \\
\text { SE }\end{array}$ & $\begin{array}{l}0.7 \mathrm{mi} \\
0.6 \mathrm{mi}\end{array}$ & $\begin{array}{l}\text { TSP } \\
\text { SO2 } \\
\text { NOX }\end{array}$ & $\begin{array}{r}218,649 \\
6,678 \\
4,436\end{array}$ \\
\hline (Coke Plant) & $\begin{array}{c}\text { (ivameoki \& Edwards- } \\
\text { ville) }\end{array}$ & $E$ & $1.0 \mathrm{mi}$ & $\mathrm{CO}$ & 18,630 \\
\hline $\begin{array}{l}\text { Tri-state Regional } \\
\text { Port District }\end{array}$ & 2801 Rock Rd. & NWW & $2.1 \mathrm{mi}$ & TSP & 2,700 \\
\hline
\end{tabular}


2. Mobile Sources that may Influence the Site:

Names of Roadways:

Type:

Distance of Roadway from Site ( $\mathrm{ft}$ )

Composition of Roadway

Number of Traffic Lanes

Average Daily Traffic

Average Vehicle Speed (mph)

One or Two Way Traffic

Number of Parking Lanes

Are Parking Lanes Used

For Traffic Part of Day?

Is Dust Visibiity Retained

Does Roadway Have Curb

20th St.

Arterial Street

$15 \mathrm{~m}$

Asphalt

4

8800

30

Two

None

$\mathrm{N} / \mathrm{A}$

Yes

Yes

3. Area Sources that may Influence the Site:

\section{Direction \\ From Site}

Gravel Parking Lot NW

St. Louis Area

SW
Distance

From Site

50-75 ft.

5 miles
Omaha

Local Street

$12 \mathrm{~m}$

Asphalt

4

$<1000$

20

Two

One

Yes

No

No
Pollutant

TSP

All 
D. Topography/Obstructions

1. General Characteristics Over a 2 Mile Radius From the Site: Smooth

2. Topographic Features that Influence the Site: (Types - hills, valleys, depressions, bodies of water, rjdges, cliffs)

Type

$\underline{\text { Size }}$

Direction

Distance

From Site

From Site

None

3. Obstructions to Wind Flow

(Types - buildings, trees, ridges, cliffs)

Type

Size

Direction

Distance

Trees

$30 \mathrm{ft}$.

From Site

From Site

Trees

$40 \mathrm{ft}$.

North

$20 \mathrm{~m}$

$\mathrm{NE}, \mathrm{E}$

$25 \mathrm{~m}$

4. Comments

In general, a number of trees scattered throughout the residential area.

E. Distance to the nearest National Weather Service (NWS) Site.

Here are the distances and directions to the nearest NWS observing site from the inhalable particulate monitors in Granite City. Distances are in statute miles; directions are degrees from true north.

Site Address

2001 East 20th
Nearest NWS Site

Lambert Airport/

St. Louis (STL)
Direction and Distance to NWS

$285^{\circ}$ at $13 \mathrm{mi}$. (W) 


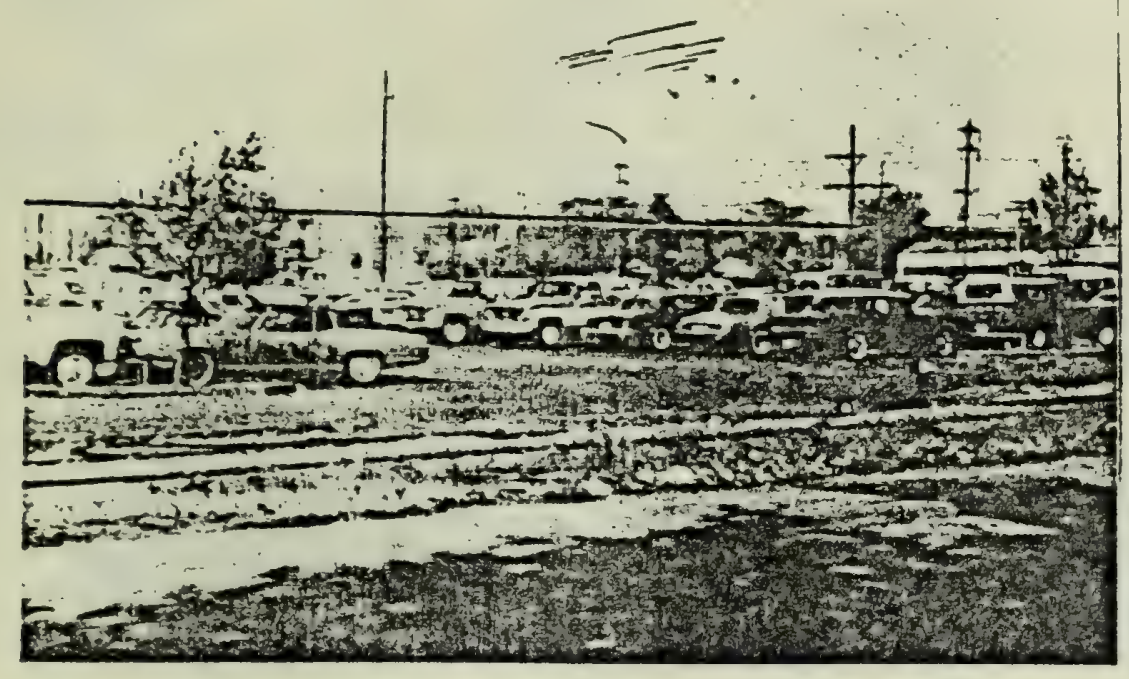

Granite City (Dallas Res.) North

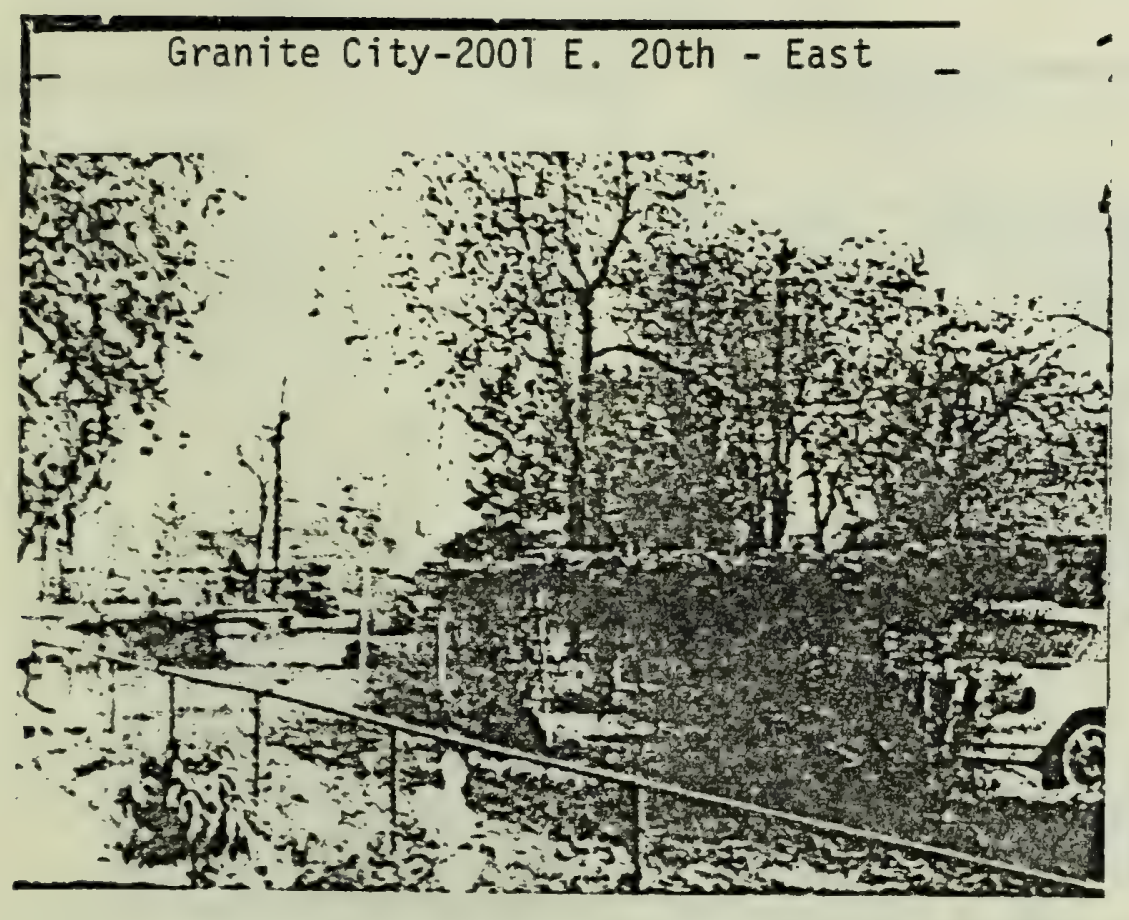

Granite City (Dallas Res.) East 


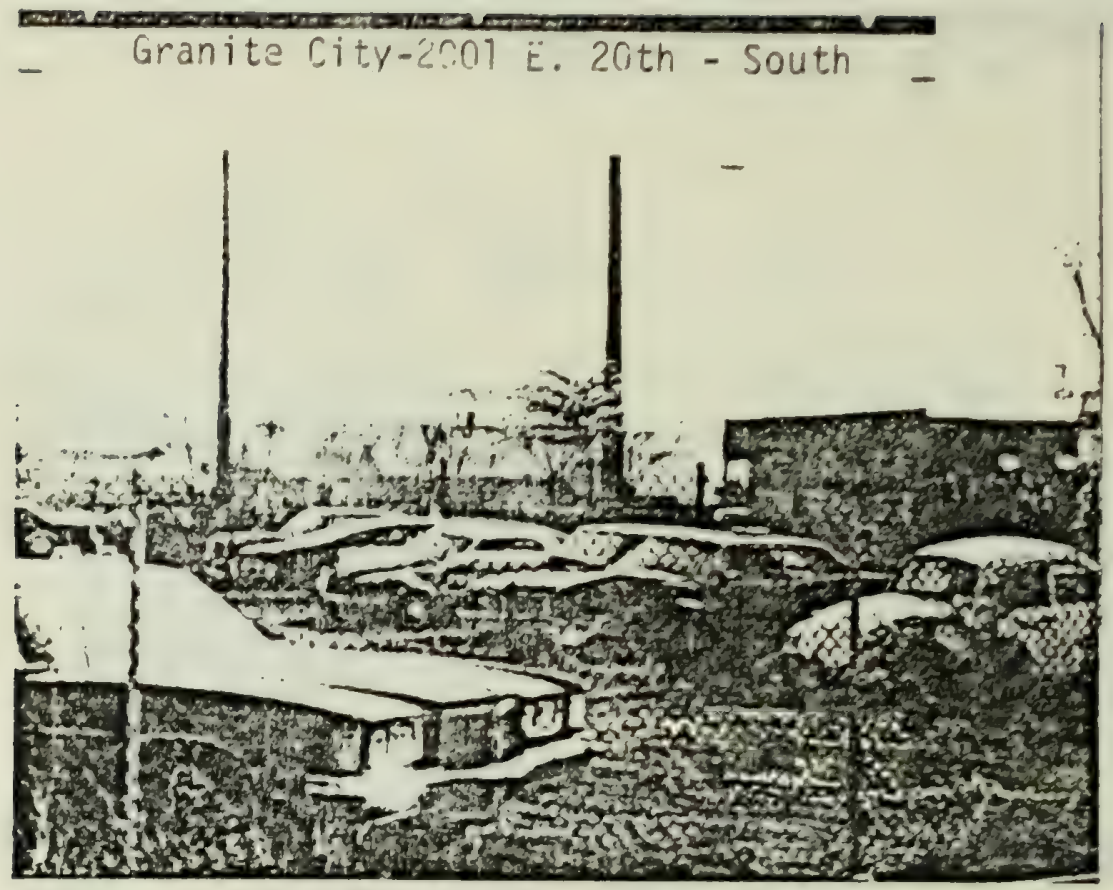

Granite City (Dallas Res.) South

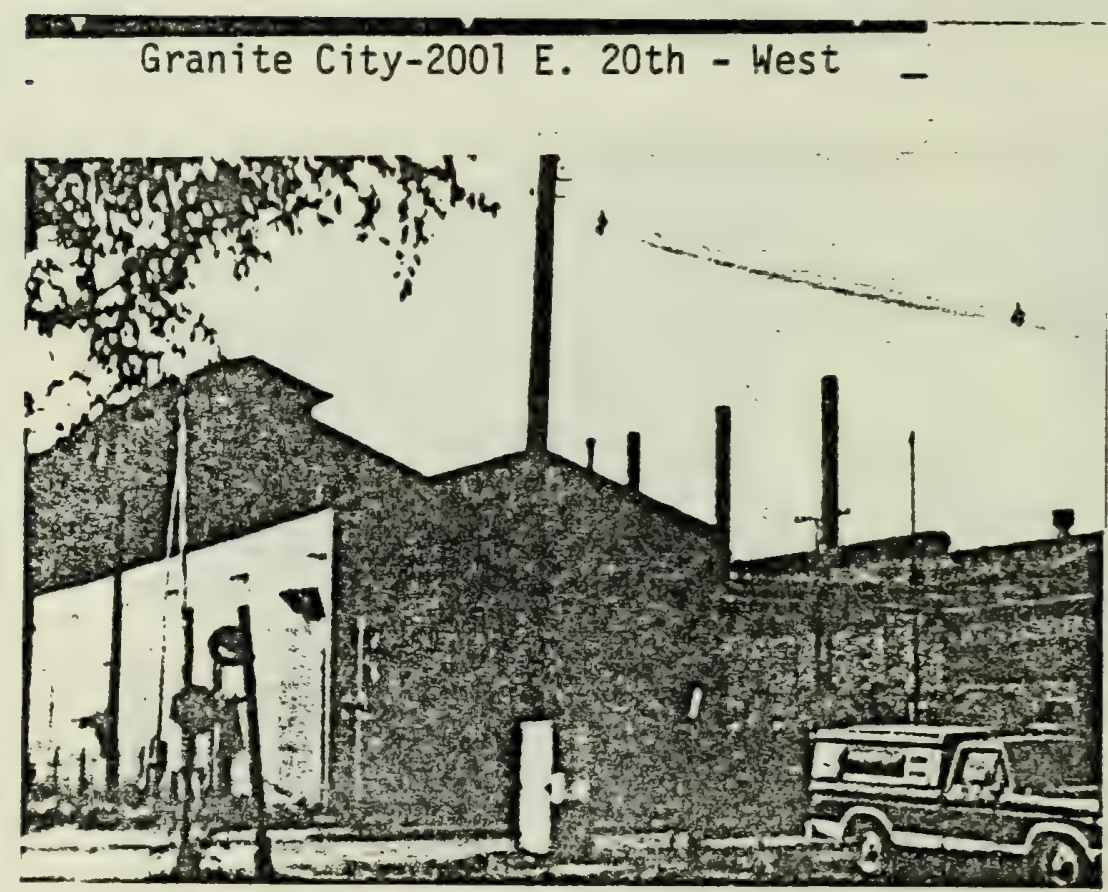

Granite City (Dallas Res.) West 
Total Suspended Particulate $\mu \mathrm{g} / \mathrm{m}^{3}$

$\begin{array}{clr}\text { Particulate: Minerals } 110.2 & \text { Combustion } & 18.4 \\ \text { Biological trace Other } & 75.5 \\ \text { Low temperature ashing, \% loss } & 8.4\end{array}$
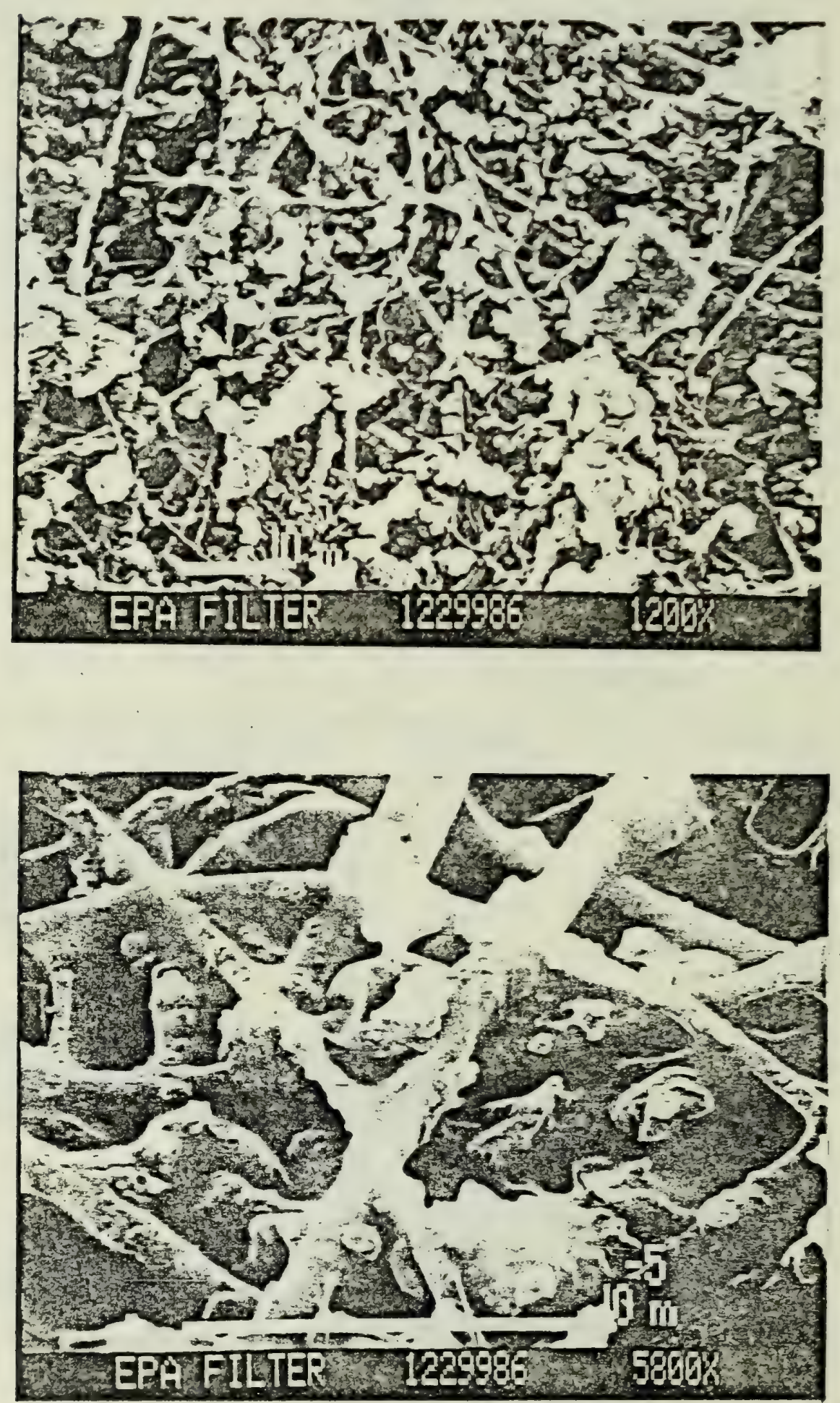


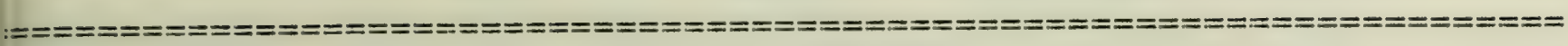

\section{ILTER NUMEEF : 1229987}

OLLECTION DATE：05, 22181

1

$\begin{aligned} \text { CIIMFONENT } & \end{aligned}$
NETWIFK :

SITE: DE.JECTIVE MAG. : 25

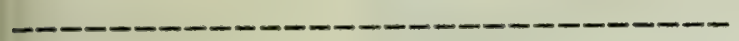

MINEFALS

RLIAFTZJFELDSFAFI:

LIMESTIINE

IFON OXIDES

DTHEF MINEFIALS

DOME:LSTION FRIDIIIETS

SDITT

BLASSY FLYASH SFHEFES

EIOLOUILAL

17. 0

2. $5-57.6$

2. $5-40.7$

13.9

1. $8-20.4$

FIAUIIE

AVEFALE

7. 0

$1 \% 1$

2. $5-91.5$

WEI GHT Number

FEFIEENT Percent

\section{FILLEN \& SFIREE \\ FLANT TISOIIE \\ STAFICH}

11.1
23.5
12.3
5. $1-20.4$
$3.6-31.5$
10. $2-14.4$

3
9
$<1$

\section{ELELLANEDIS \\ FEFFIDUIS METAL \\ FUUEEEF:}

$-7$

31

0

29
14
3
0

19

31

7

0

0

0

0

\section{FAFTICLE BIZE IISTFIEUTIIN}

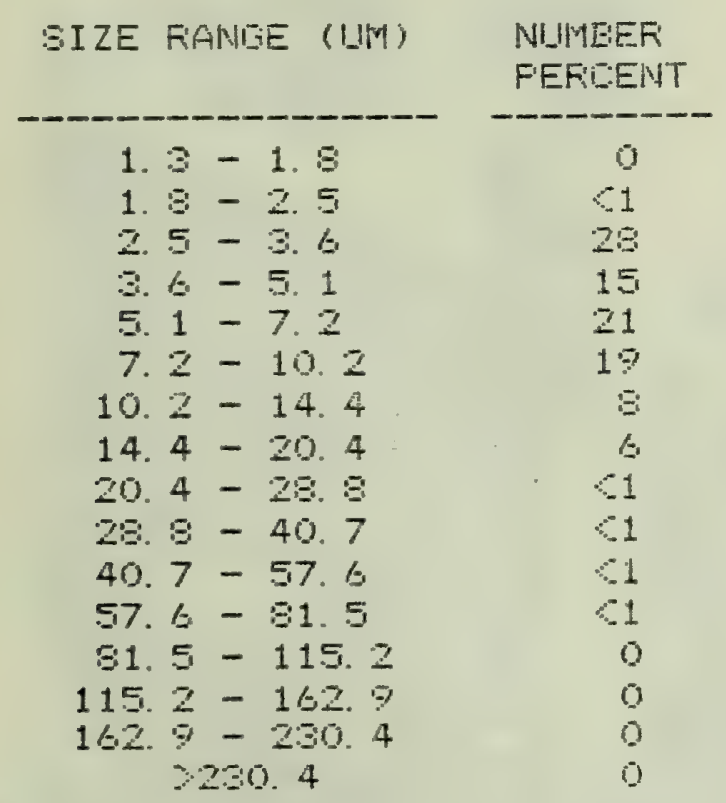

\begin{tabular}{c} 
WEIBHT \\
FEREENT \\
\hline 0 \\
1 \\
1 \\
1 \\
2 \\
4 \\
6 \\
12 \\
3 \\
10 \\
16 \\
45 \\
0 \\
0 \\
0 \\
0
\end{tabular}

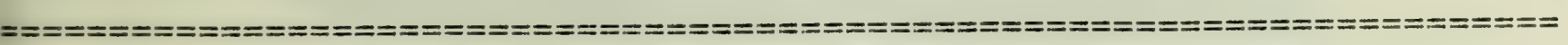
COMMENTS: IFUN ANLIGR STEEL IS FRESENT EUIT IS ASOULIATEM WITH THE IFUIN OXIDE ANI NOT FFEEENT AS DIELFETE FIAFTICLES. 
Inhalable Particulate

$\mu g / \mathrm{m}^{3}$

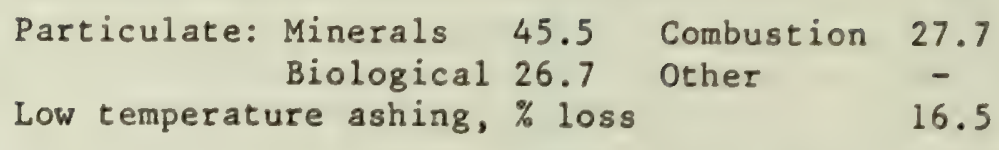
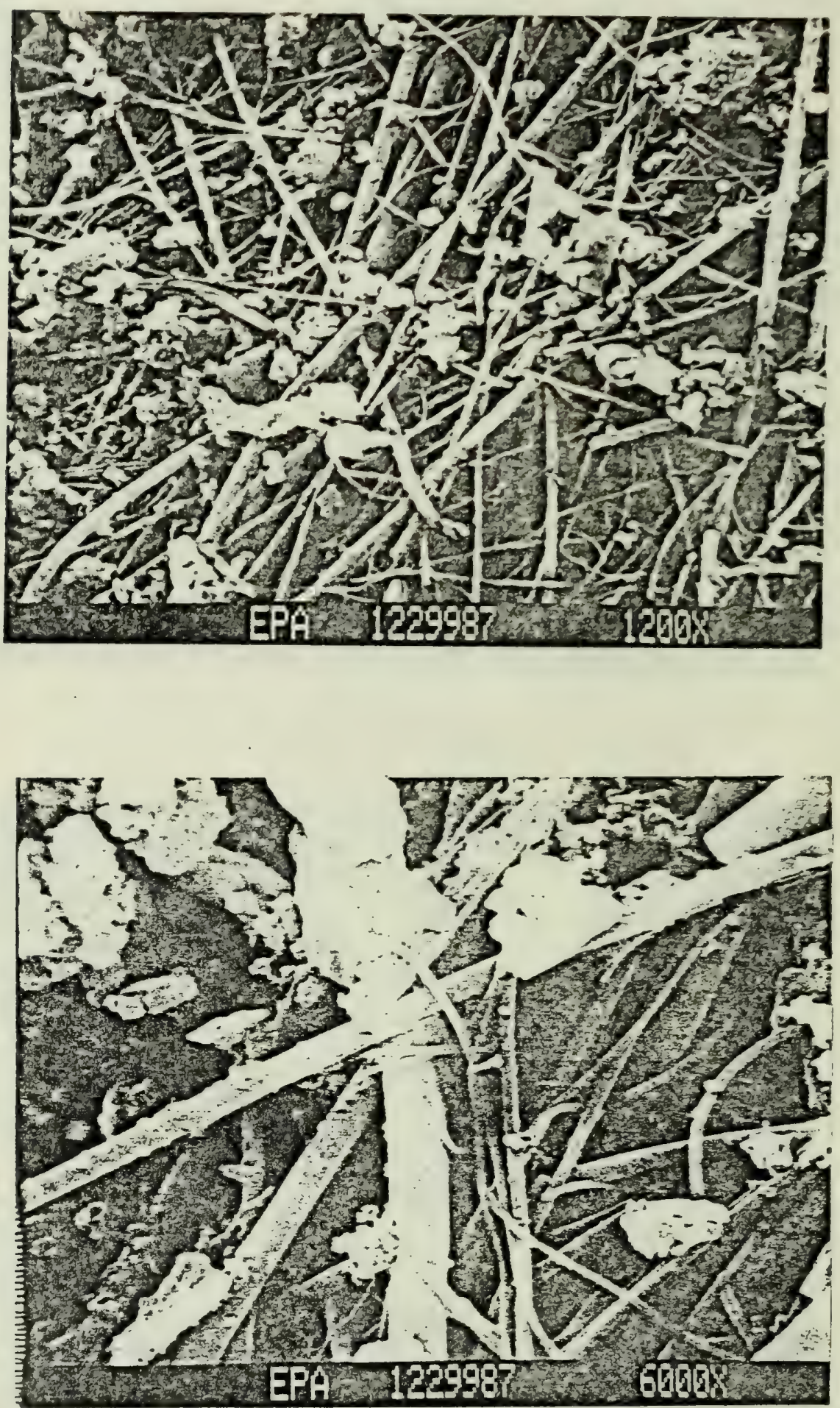


\begin{tabular}{|c|c|c|c|c|}
\hline $\begin{array}{l}\text { ILTER NUIMEER : } 1230411 \\
\text { OLLECTIION DATE : } 06 / 03 / 81 \\
======================\end{array}$ & $\begin{array}{l}\text { NETWDFK } \\
\text { SITE : } \\
\text { QR.JETTI } \\
==========\end{array}$ & \multicolumn{2}{|c|}{ GRANICITY/DALLASRES } & \\
\hline COMMGINENT & $\begin{array}{l}\text { AVERIAIGE } \\
\text { FAFT. } S I Z E\end{array}$ & RANGE & $\begin{array}{l}\text { WEIGHT } \\
\text { FERICEINT }\end{array}$ & $\begin{array}{l}\text { Number } \\
\text { Percent }\end{array}$ \\
\hline \multicolumn{5}{|l|}{ MINERALS } \\
\hline QUAARTZ $/ F E L$ ISFARS: & 89.0 & $9.0-289.3$ & 41 & 21 \\
\hline LIMESTIDNE & 45.8 & 6. $4-204.6$ & 4 & 29 \\
\hline IRON OXIDES & 91.5 & $9.0-289.3$ & 11 & 1 \\
\hline OTHER MINEFAAL_S & & & 0 & 0 \\
\hline COMEUSTION FFOCHLTE & & & & \\
\hline $\begin{array}{l}\text { SODT } \\
\text { GLASSY FLYASH SFHEFES }\end{array}$ & 84. 7 & $9.0-289.3$ & 0 & $\begin{array}{r}41 \\
0\end{array}$ \\
\hline \multicolumn{5}{|l|}{ BIOLOGICAL. } \\
\hline POLILEN \& SFORES & 30.9 & $25.6-36.2$ & $<1$ & $<1$ \\
\hline $\begin{array}{l}\text { PLANT TISGUE } \\
\text { STARL:H }\end{array}$ & 167.1 & $72.3-289.3$ & $\begin{array}{l}8 \\
0\end{array}$ & 1 \\
\hline I SCELLANEOUS & & & & \\
\hline $\begin{array}{l}\text { FEFFROUS METAL } \\
\text { RUEEEE }\end{array}$ & 85. 0 . & 12. $8-28 \% .3$ & $\begin{array}{r}27 \\
0\end{array}$ & $\begin{array}{l}6 \\
0\end{array}$ \\
\hline
\end{tabular}

FAFT ICLE SIZE DISTRIEUTIONN

\begin{tabular}{|c|c|c|}
\hline SIZE RIANGE (LIM) & $\begin{array}{l}\text { NUMMEEF } \\
\text { FEFICENT }\end{array}$ & $\begin{array}{l}\text { WEIGHT } \\
\text { FEFICENT }\end{array}$ \\
\hline $3.2-4.5$ & 0 & 0 \\
\hline $4.5-6.4$ & 0 & 0 \\
\hline 6. $4-9.0$ & 4 & $<1$ \\
\hline $9.0-12.8$ & 30 & $<1$ \\
\hline $12.8-18.1$ & 9 & $<1$ \\
\hline $18.1-25.6$ & 19 & $<1$ \\
\hline $25.6-36.2$ & $\epsilon$ & $<1$ \\
\hline $36.2-51.1$ & 8 & $<1$ \\
\hline $51.1-72.3$ & 14 & 3 \\
\hline $72.3-102.3$ & 2 & $<1$ \\
\hline $102.3-144.6$ & $<1$ & $<1$ \\
\hline $144.6-204.6$ & 4 & 23 \\
\hline $204.6-28 \% 3$ & 5 & 71 \\
\hline $289.3-409.1$ & 0 & 0 \\
\hline $40 \% .1-578.6$ & 0 & 0 \\
\hline$>579.6$ & 0 & 0 \\
\hline
\end{tabular}


$\begin{array}{cclr}\text { Particulate: Minerals } 157.9 & \text { Combustion } & 25.4 \\ \text { Biological 22.6 } & \text { Other } & 76.1 \\ \text { Low temperature ashing, \% loss } & 6.5\end{array}$
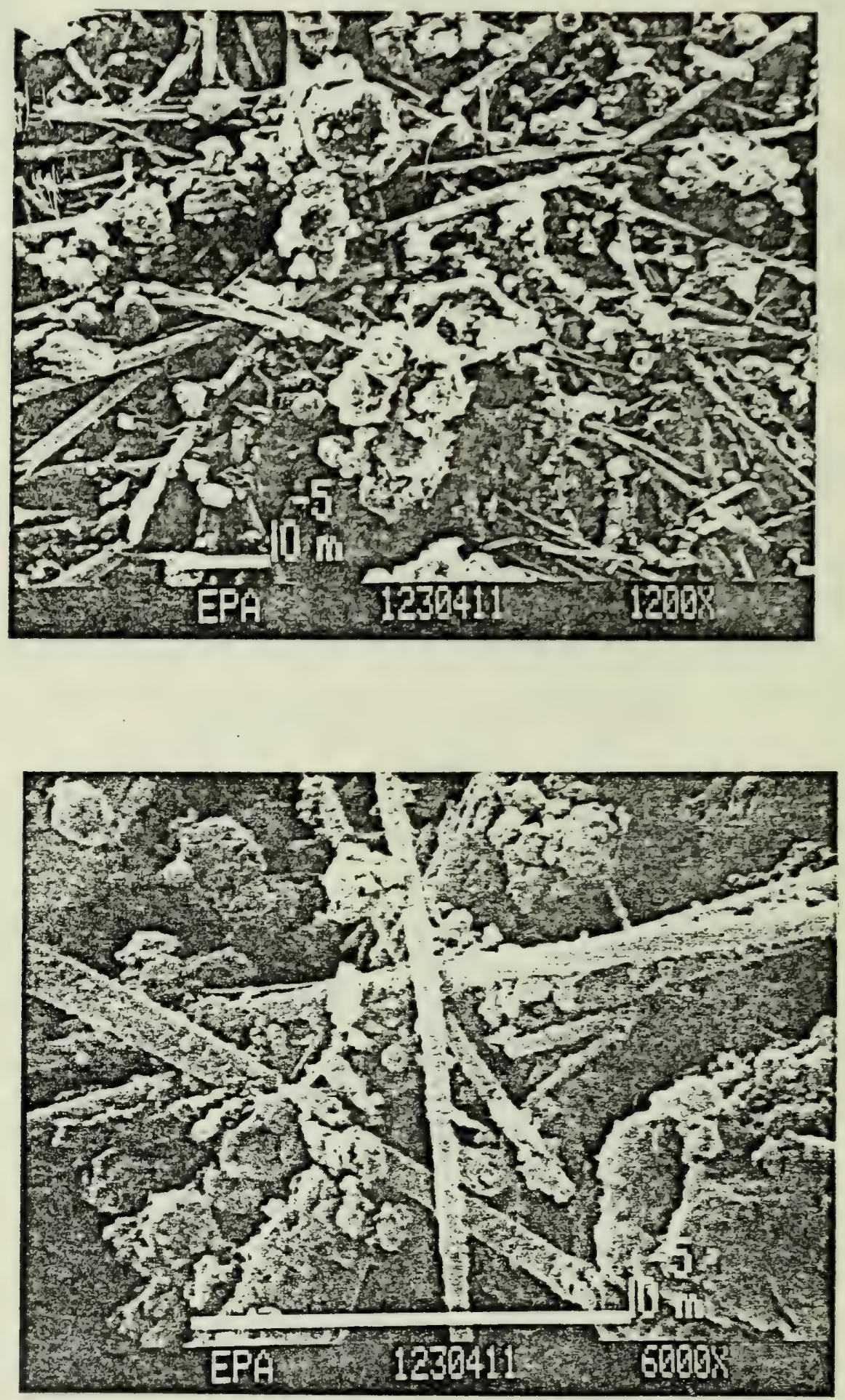

Particulate: Minerals 176
Combustion 74.3
Biological 8
Other
132.9
Low temperature ashing, \% loss
4.0
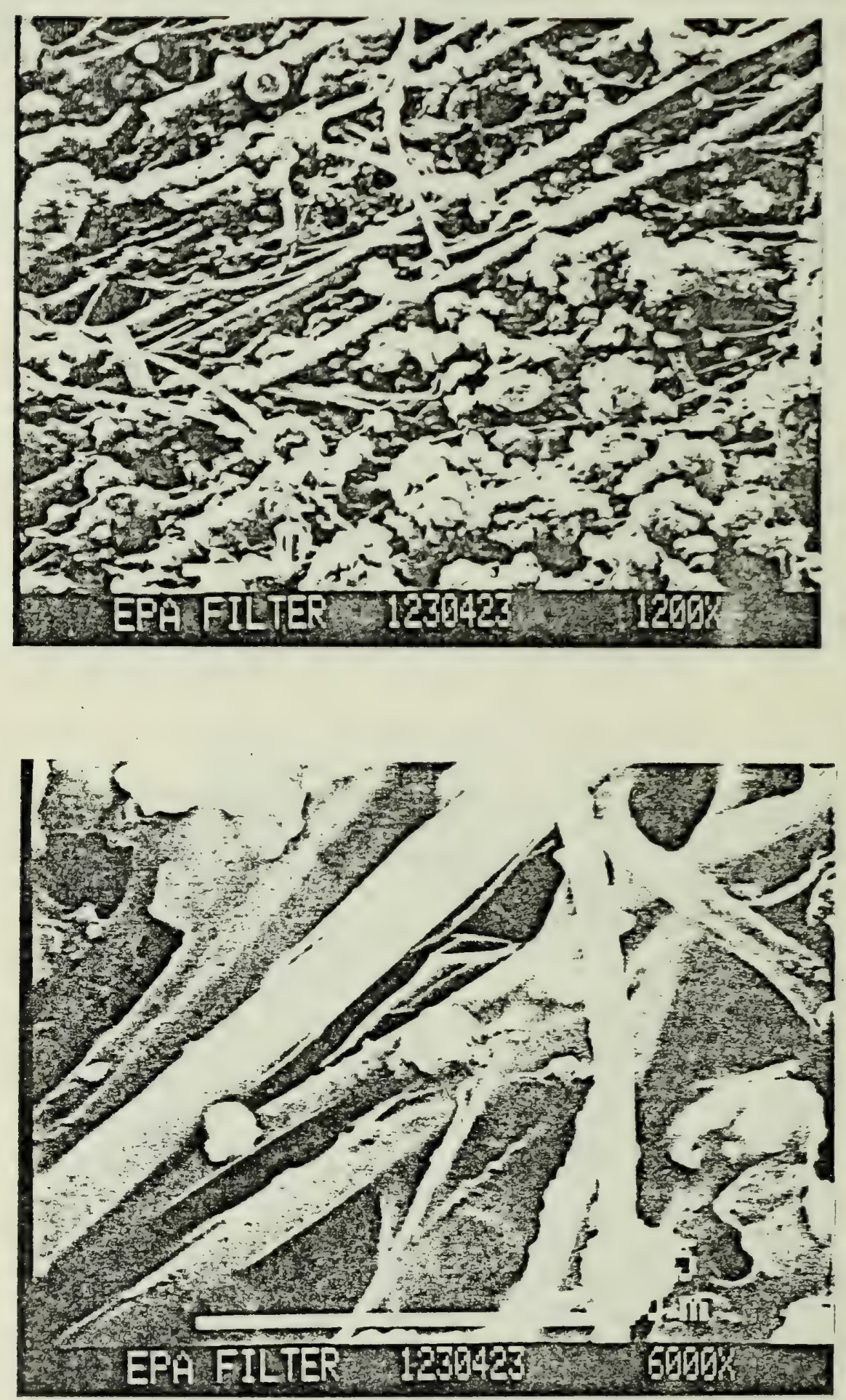


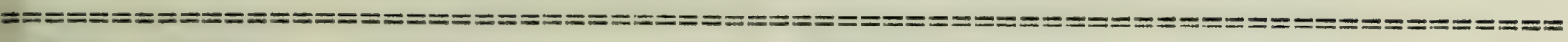

ILTEF NUMEER : 1290422

DLLECTION DATE : OS/15/81

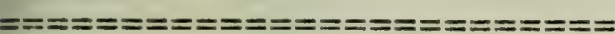
COMFINENT
NETWIDFE :

SITE : DE.IELTIVE MAL: : 25

\section{GFIANIEITY/LIALLAEFES}

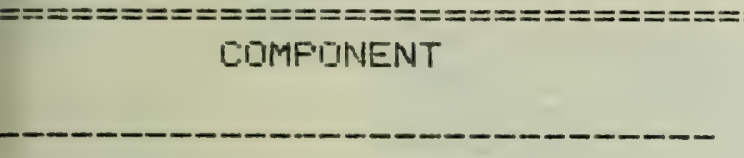

\section{AVEFIAIDE \\ FART. SIZE}

MINEFIALS
DIJAFTZ IFELISFAF:S

LIMESTONE

IFIDN QXILES

DTHER MINEFALS

15. 7

\%. 3

11. 6

20.1

SIIDT

GLASSY FLYASH SFHEFES
FIANISE

2. $5-57.6$
1. $8-26.9$
2. $5-40.7$

2. $5-81.5$

-1-20. 4

3. $6-23$.
WEICHT Number FEFICENT Percent

EIDLITIIIEAL

FIILLEN \& EFIRES

FLANT TISGIIE

STAFIEH
11. 1

12. 3

12.6

$2.5-57.6$

36

22

13

0

25

0

17

\section{MISLELLANEDUS:}

FEFIFIUIS METAL

FilIEEER

$\begin{array}{rr}1 & 1 \\ 2 & 2 \\ 0 & 0\end{array}$

\section{FAFTILLE SIZE IISTFIEUITION}

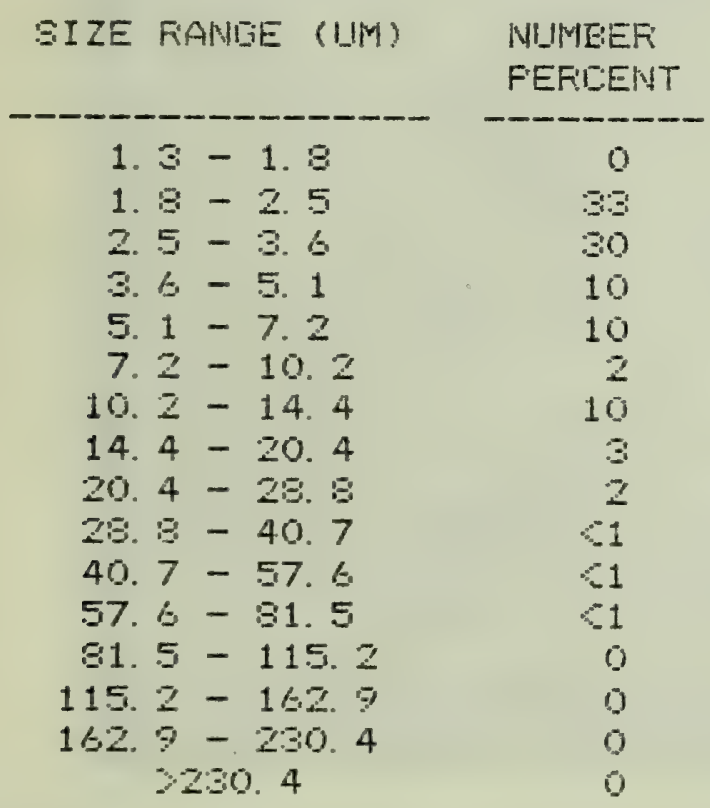

WEIGHT

FERILENT

0
1
1
1
2
1
16
9
27
11
13
19
0
0
0
0


Particulate: Minerals 76.5 Combustion 168.3

$\begin{array}{ll}\text { Biological } 7.7 \text { Other } & - \\ \text { re ashing, } \% \text { loss } & 4.8\end{array}$

Low temperature ashing, \% loss 4.8
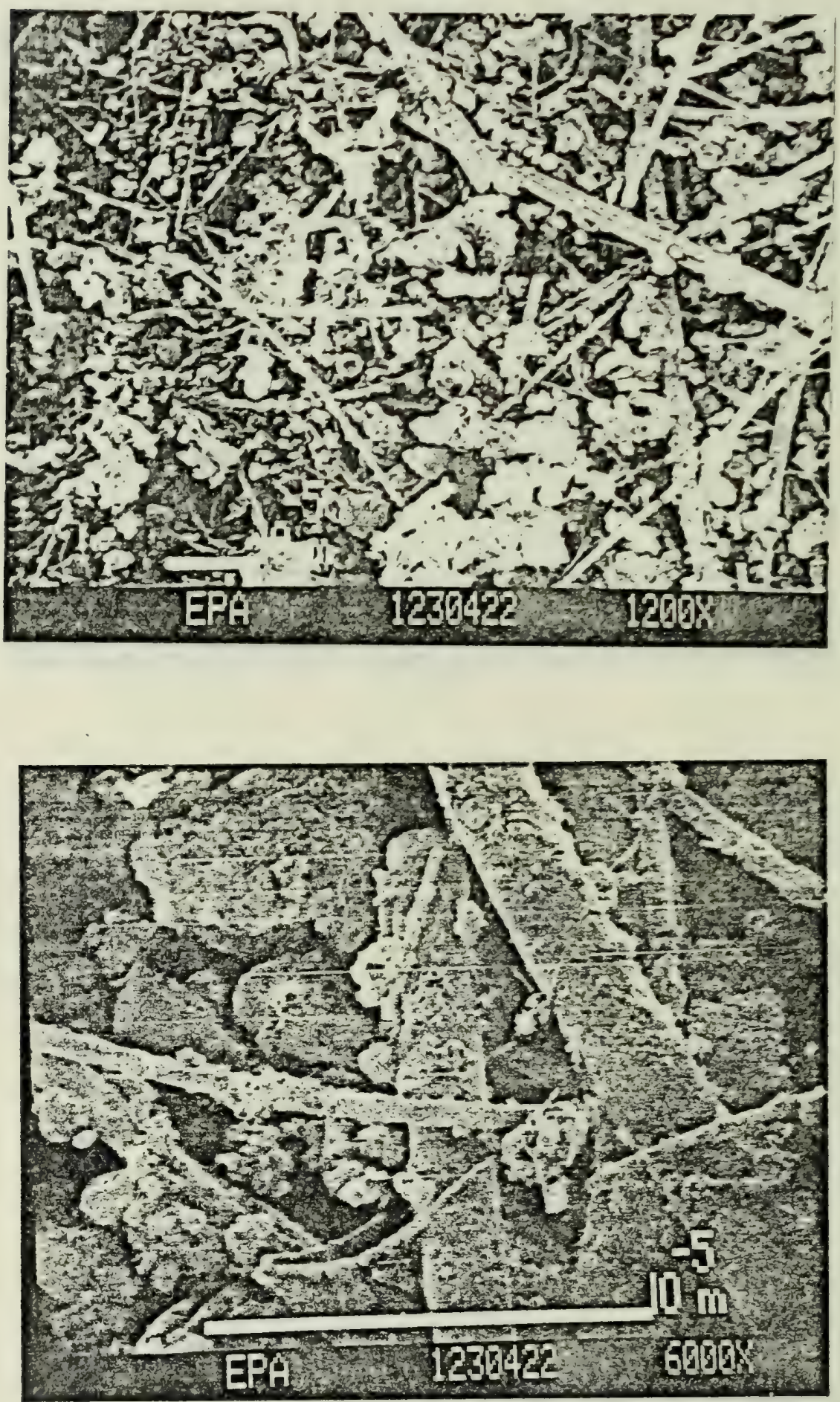


\begin{tabular}{|c|c|c|c|c|}
\hline $\begin{array}{l}\text { ILTER NLIMBER : } 1277784 \\
\text { OLLECTION DATE : } 08 / 14 / 81 \\
=======================\end{array}$ & $\begin{array}{l}\text { NETWOKK } \\
\text { SITE. : }\end{array}$ & \multicolumn{2}{|c|}{$\begin{array}{l}\text { IFFANILITY/TALLAEFE: } \\
\text { AR. } \quad: 25\end{array}$} & \multirow[b]{2}{*}{$\begin{array}{l}\text { Number } \\
\text { Percent }\end{array}$} \\
\hline COMFONENT & $\begin{array}{l}\text { AVEFAIIE } \\
\text { FART. SIZE }\end{array}$ & RANISE & $\begin{array}{l}\text { WEI I.SHT } \\
\text { FEFTEEIVT }\end{array}$ & \\
\hline \multicolumn{5}{|l|}{ MINERALS } \\
\hline QUARTZ Z FEI_חSFAR: & 17.3 & 5. $1-40.7$ & e & 9 \\
\hline LIMESTUNE & 12. 4 & 2. $5-40.7$ & 3 & 27 \\
\hline IRON OXIDES & 12. 3 & 3. $6-28.8$ & 7 & 9 \\
\hline OTHER MINERAL_S & & & 0 & 0 \\
\hline \multicolumn{5}{|l|}{ COMEIISTION FRODUITS } \\
\hline $\begin{array}{l}\text { BDOT } \\
\text { GI_ASSY FI_YASH SFHEFES }\end{array}$ & 25.3 & 1. $8-16 z .7$ & $\begin{array}{r}53 \\
0\end{array}$ & $\begin{array}{r}53 \\
0\end{array}$ \\
\hline \multicolumn{5}{|l|}{ BIOLOGICAL- } \\
\hline $\begin{array}{l}\text { FLANT TISGIIE } \\
\text { STARL:H } \\
\text { HAIR }\end{array}$ & $3 \% 1$ & 14. $4-81.5$ & $\begin{array}{r}29 \\
0 \\
0\end{array}$ & $\begin{array}{l}2 \\
0 \\
0\end{array}$ \\
\hline MI SLELLLANEOULS & & & & \\
\hline $\begin{array}{l}\text { FEFREOUE METAL- } \\
\text { RUEEER }\end{array}$ & - & & $\begin{array}{l}0 \\
0\end{array}$ & $\begin{array}{l}0 \\
0\end{array}$ \\
\hline
\end{tabular}

FAFTICLE SIZE RISTRIEIUTION

\begin{tabular}{ccc} 
SIZE RANGE (IIM) & $\begin{array}{c}\text { NIIMEEF } \\
\text { FERLENT }\end{array}$ & $\begin{array}{c}\text { WEIGHT } \\
\text { PEFICENT }\end{array}$ \\
\hline $1.3-1.8$ & 0 & -8 \\
$1.8-2.5$ & 13 & 61 \\
$2.5-3.6$ & 35 & 61 \\
$3.6-5.1$ & 12 & 61 \\
$5.1-7.2$ & 13 & 2 \\
$7.2-10.2$ & 12 & 2 \\
$10.2-14.4$ & 5 & 5 \\
$14.4-20.4$ & 4 & 12 \\
$20.4-28.8$ & 4 & 9 \\
$28.8-40.7$ & 1 & 22 \\
$40.7-57.6$ & 61 & 0 \\
$57.6-31.5$ & 61 & 4 \\
$81.5-1.5 .2$ & 0 & 0 \\
$115.2-162.9$ & 61 & 0 \\
$162.9-230.4$ & 0 & 0
\end{tabular}


$\begin{array}{cclc}\text { Particulate: Minerals } & 40.9 & \text { Combustion } & 120.3 \\ \text { Biological } & 65.8 & \text { Other } & - \\ \text { Low temperature ashing, } \% \text { loss } & & 8.8\end{array}$
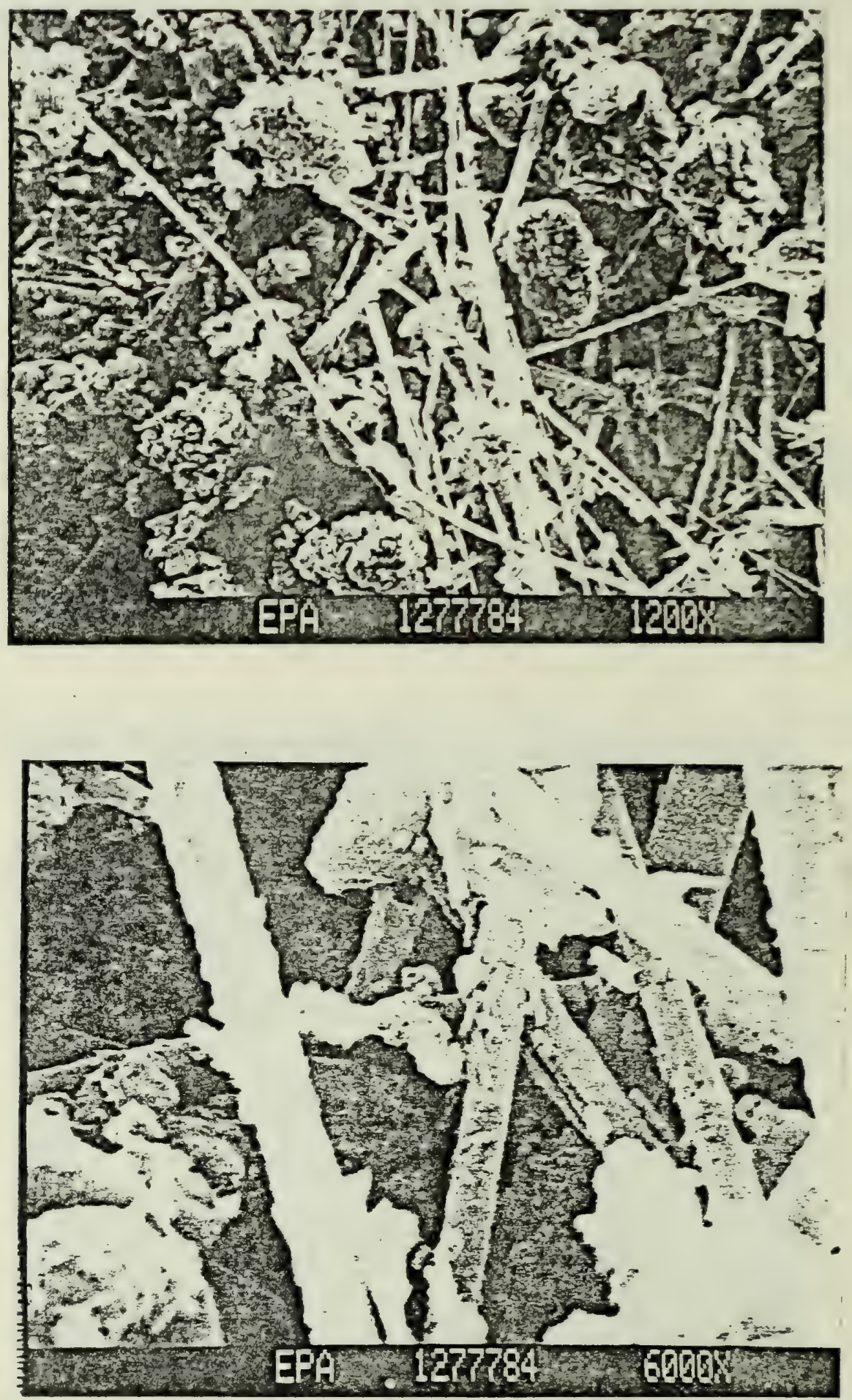


\begin{tabular}{|c|c|c|c|c|}
\hline 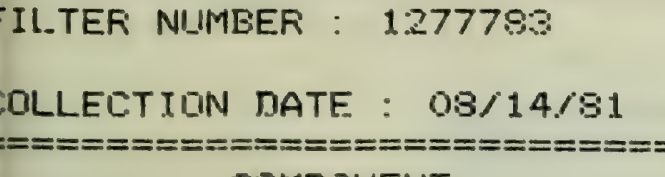 & $\begin{array}{l}\text { NETWIIFIK } \\
\text { SITE : } \\
\text { OE.JEC:TIV } \\
==========\end{array}$ & $\begin{array}{l}\text { GFIAIVICIT } \\
\text { 13. : } 25 \\
========\end{array}$ & $\begin{array}{l}\text { DALLAASFES } \\
=========\end{array}$ & \\
\hline CIOMPQINENT & $\begin{array}{l}\text { AVERALIE } \\
\text { FAFT. SIZE }\end{array}$ & RANISE & $\begin{array}{l}\text { WEIIHTT } \\
\text { FERLEINT }\end{array}$ & $\begin{array}{l}\text { Number } \\
\text { Percent }\end{array}$ \\
\hline \multicolumn{5}{|l|}{ MINEFIALS } \\
\hline QUIAFTZ/FFI_RSFAF: & 11. 1 & 5. $1-20.4$ & 19 & 7 \\
\hline L. IMESTIONE & 10. 9 & $2.5-28.8$ & 41 & 40 \\
\hline IRUN OXIDF: & 7. 9 & 3. $6-14.4$ & 13 & 6 \\
\hline OTHER MINEFALS & & & 0 & 0 \\
\hline \multicolumn{5}{|l|}{ COMEIISTINN FRODIILTS } \\
\hline S00T & 6. 9 & 2. $5-14.4$ & 14 & 44 \\
\hline GLASSY FI_YASH SFHEFES & & & 0 & 0 \\
\hline $\begin{array}{l}\text { BIOL_DISICAL. } \\
\text { FOI_LEN \& SFOKES }\end{array}$ & & & 0 & 0 \\
\hline $\begin{array}{l}\text { FLANT TISEIJE } \\
\text { STAFC:H }\end{array}$ & 15. 9 & 5. $1-40.7$ & $\begin{array}{r}13 \\
0\end{array}$ & $\begin{array}{l}3 \\
0\end{array}$ \\
\hline \multicolumn{5}{|l|}{ MISIEELLANEGII: } \\
\hline $\begin{array}{l}\text { FEFFIDUS METAL } \\
\text { RUEBER }\end{array}$ & & & $\begin{array}{l}0 \\
0\end{array}$ & $\begin{array}{l}0 \\
0\end{array}$ \\
\hline
\end{tabular}

FAFTICIE SIIZE TISTRIEUTTION

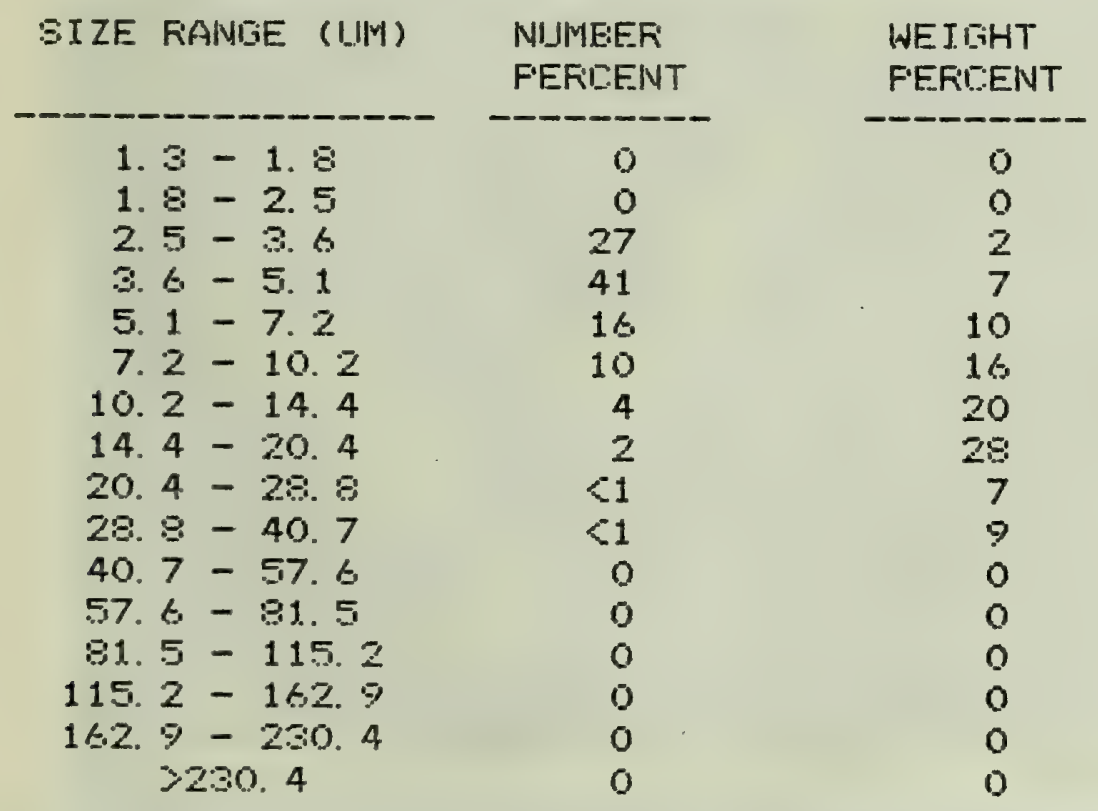


Inhalable Particulate $\quad \mu g / \mathrm{m}^{3}$
Particulate: Minerals 129.2 Combustion 24.8 Biological 23 Other -

Low temperature ashing, \% loss 15.1
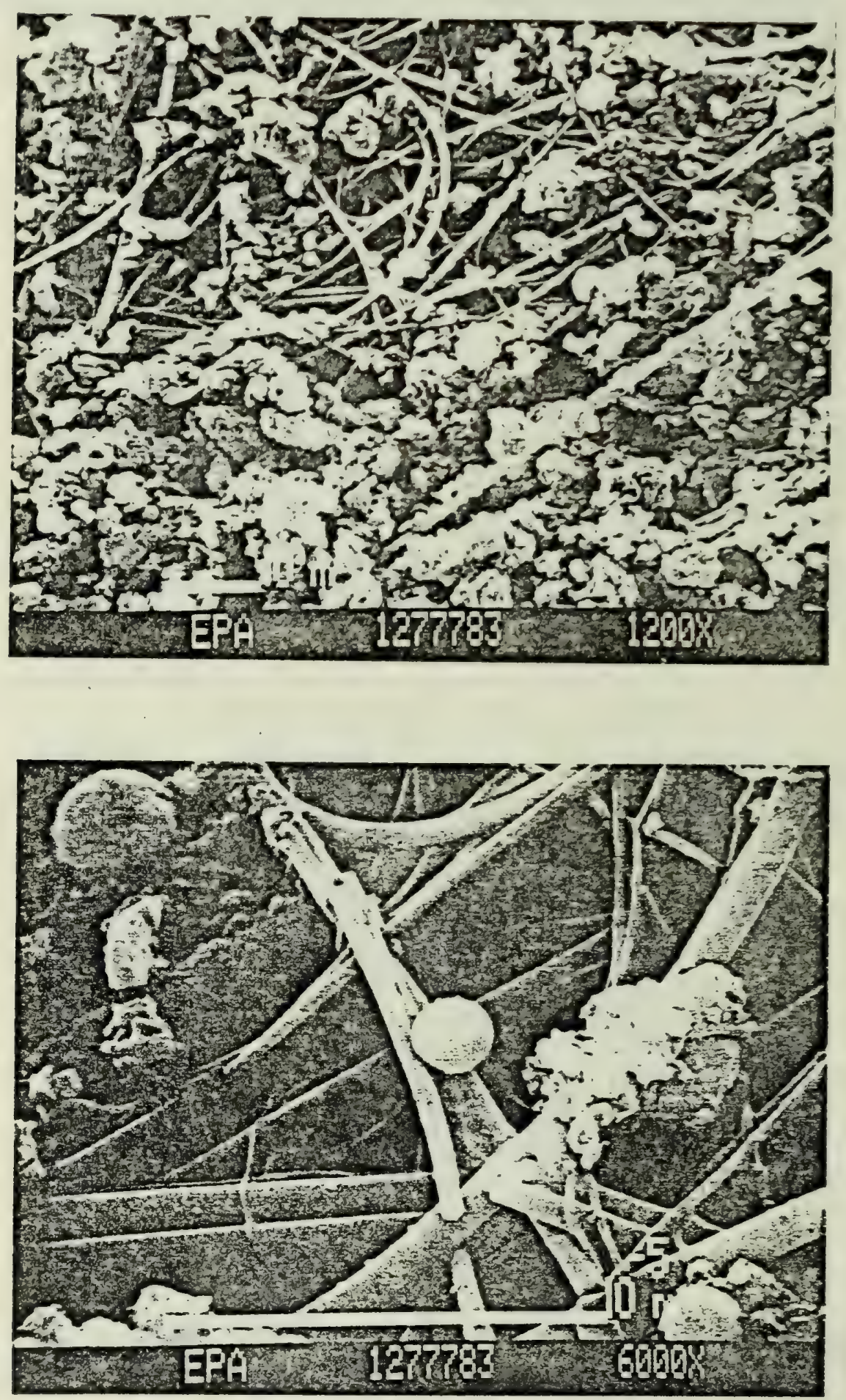

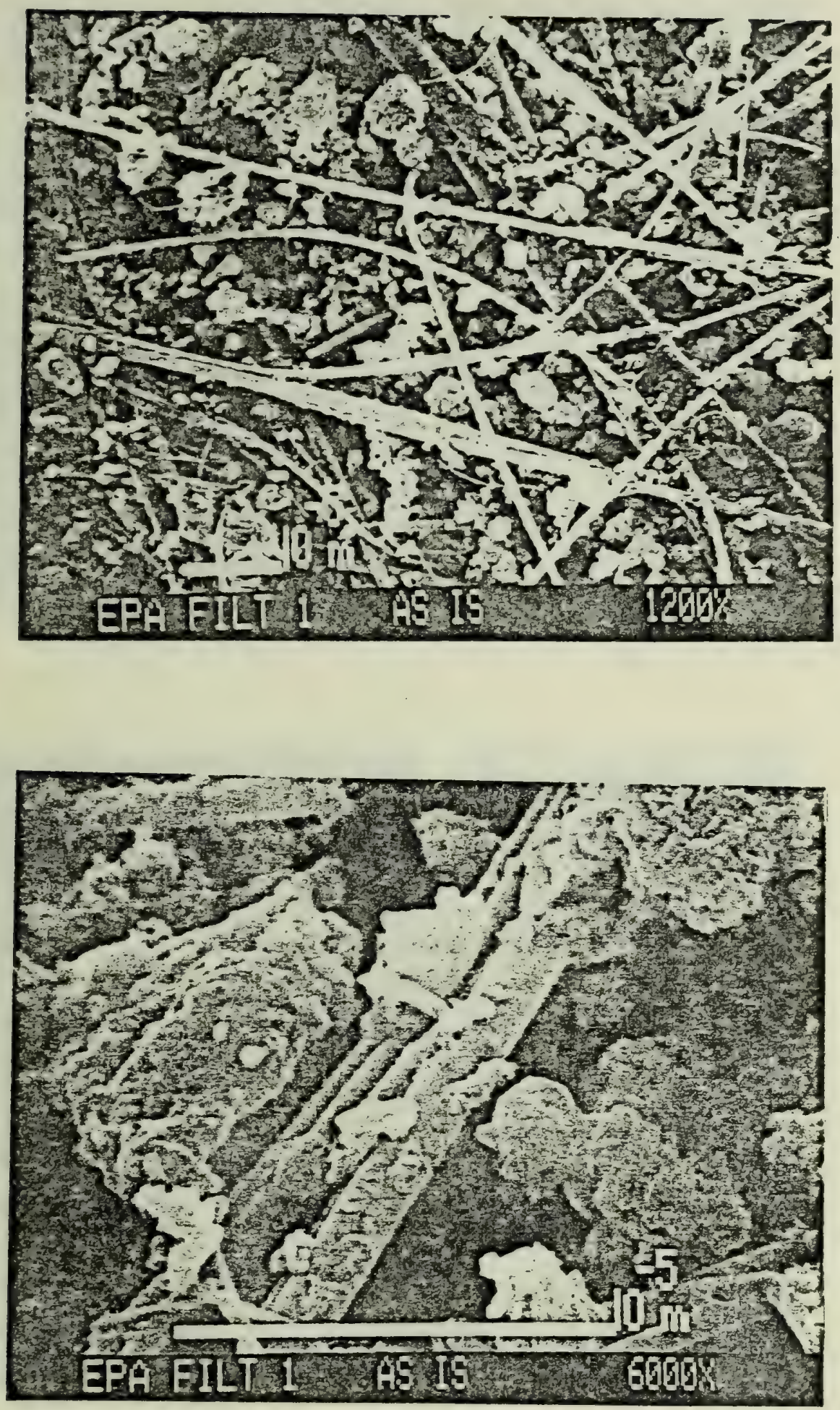

Filter 1229986 

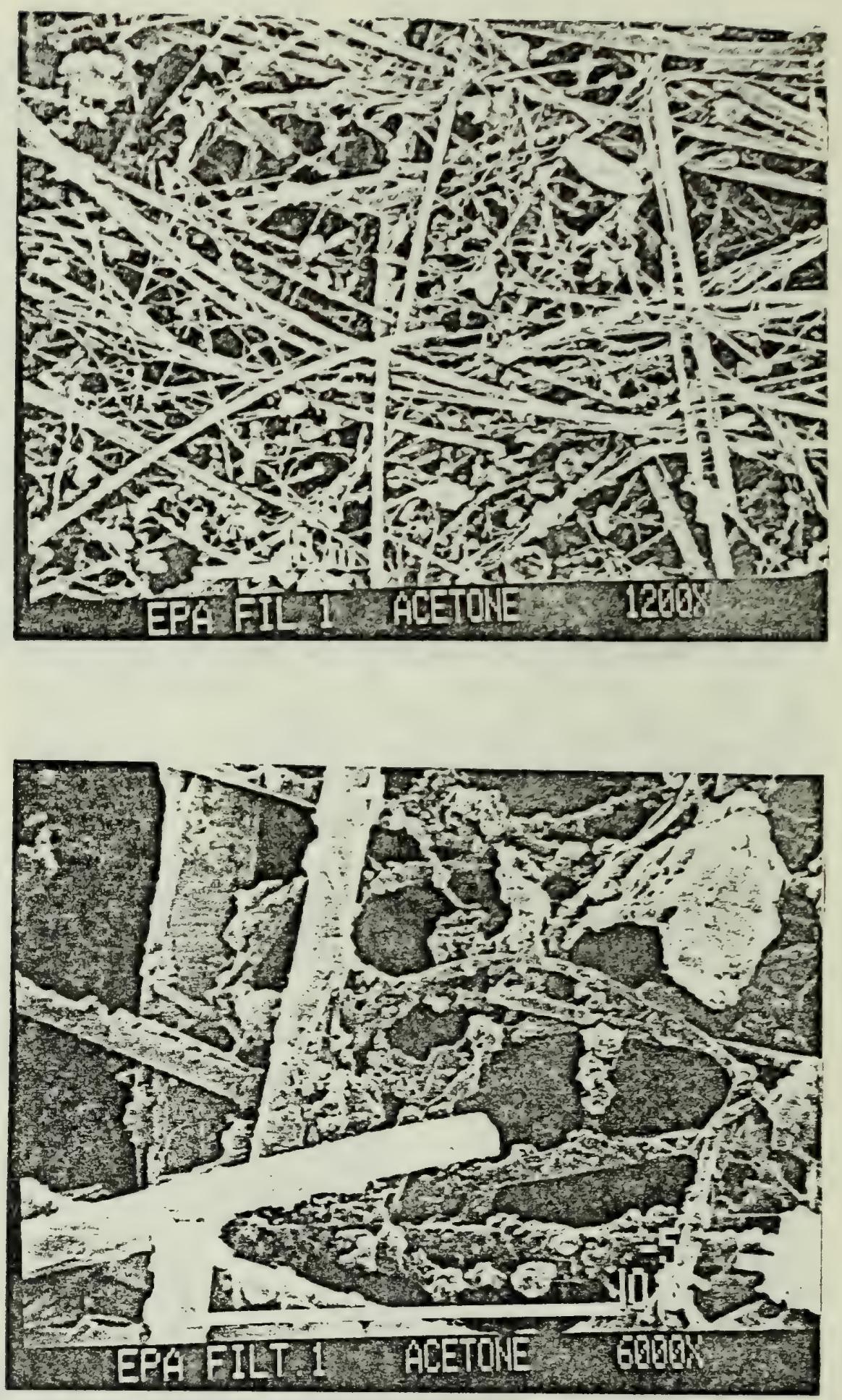

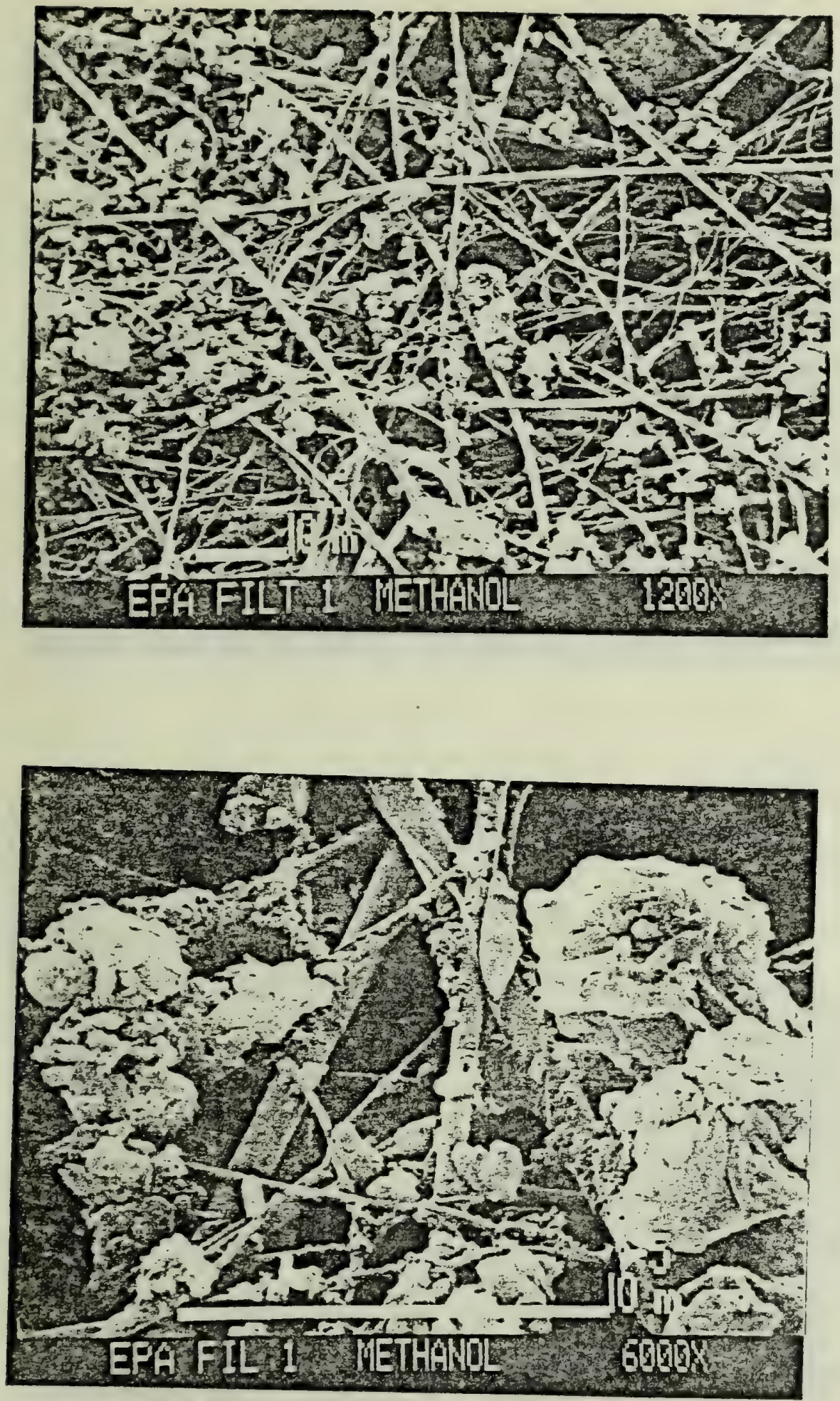

Filter 1229986 after ultrasonification in methanol 

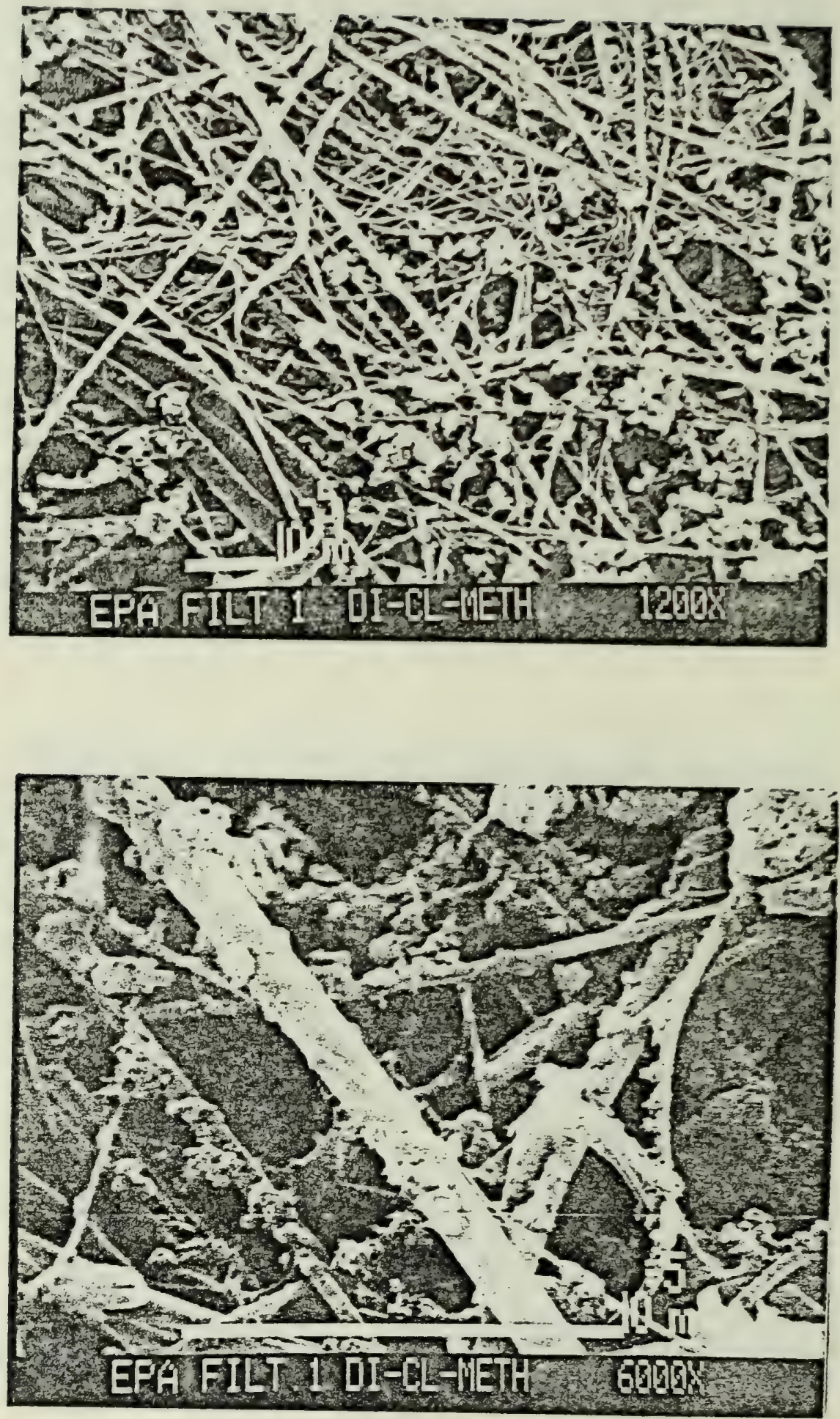

Filter 1229986 after ultrasonfication in dichtoromethane 

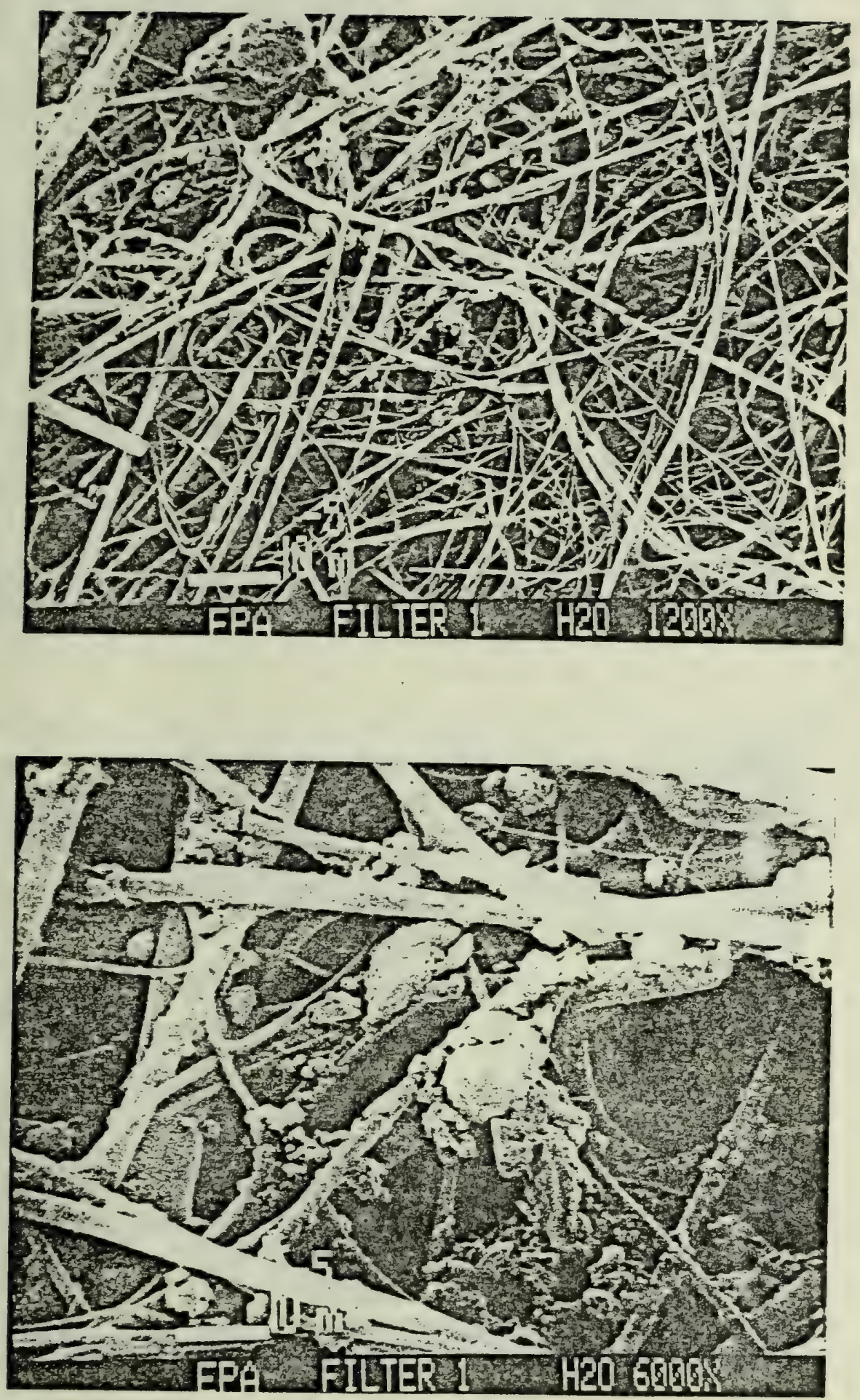

Filter 1229986 after ultrasonification in water 

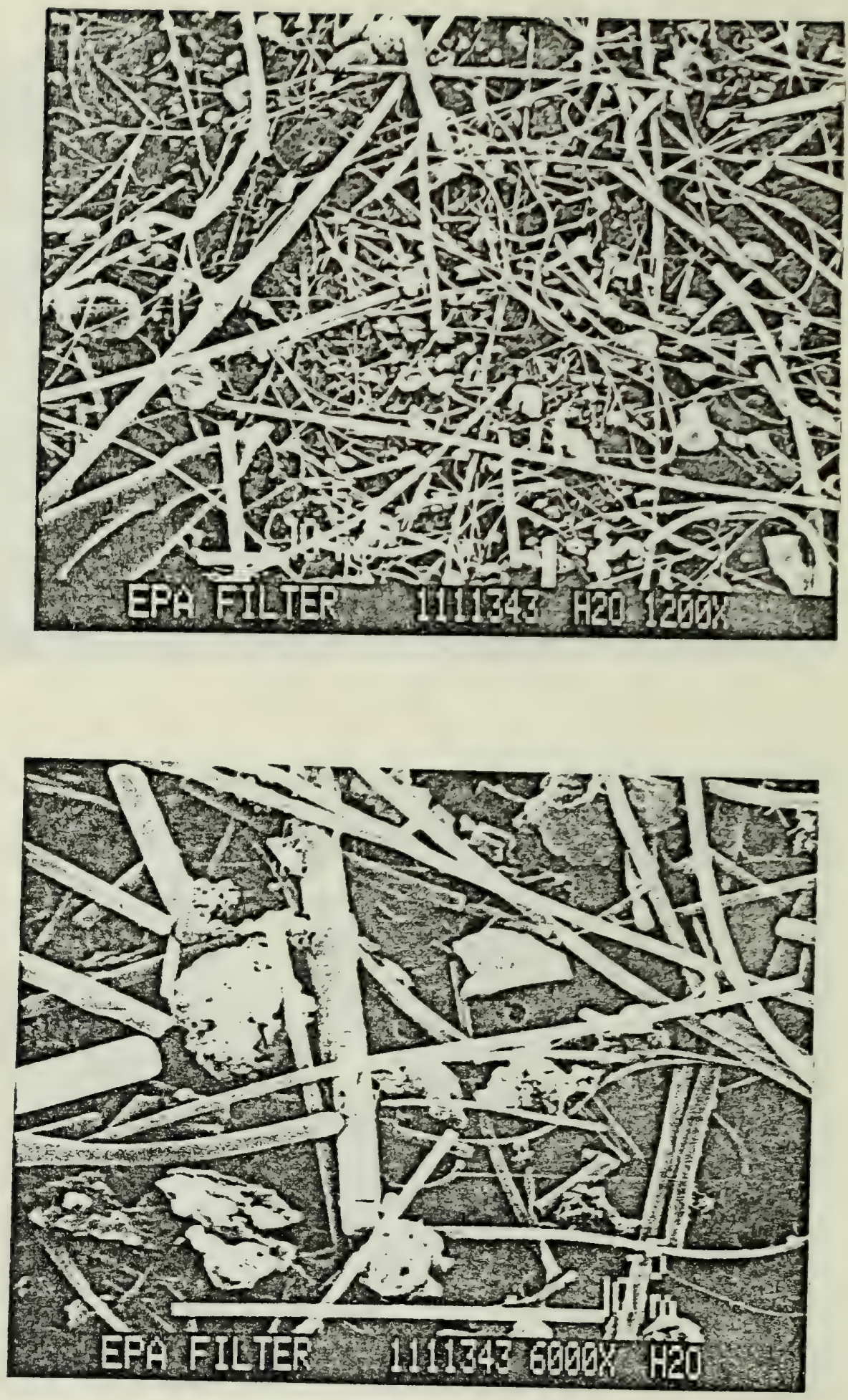

Solids collected on Nuclepore filter from ultrasonification water layer 


UNIVEASTYY OF ILLINOIS-UABANA

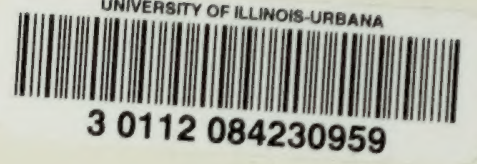

